Prepared in cooperation with the National Park Service

\title{
Assessment of Historical Water-Quality Data for National Park Units in the Rocky Mountain Network, Colorado and Montana, through 2004
}

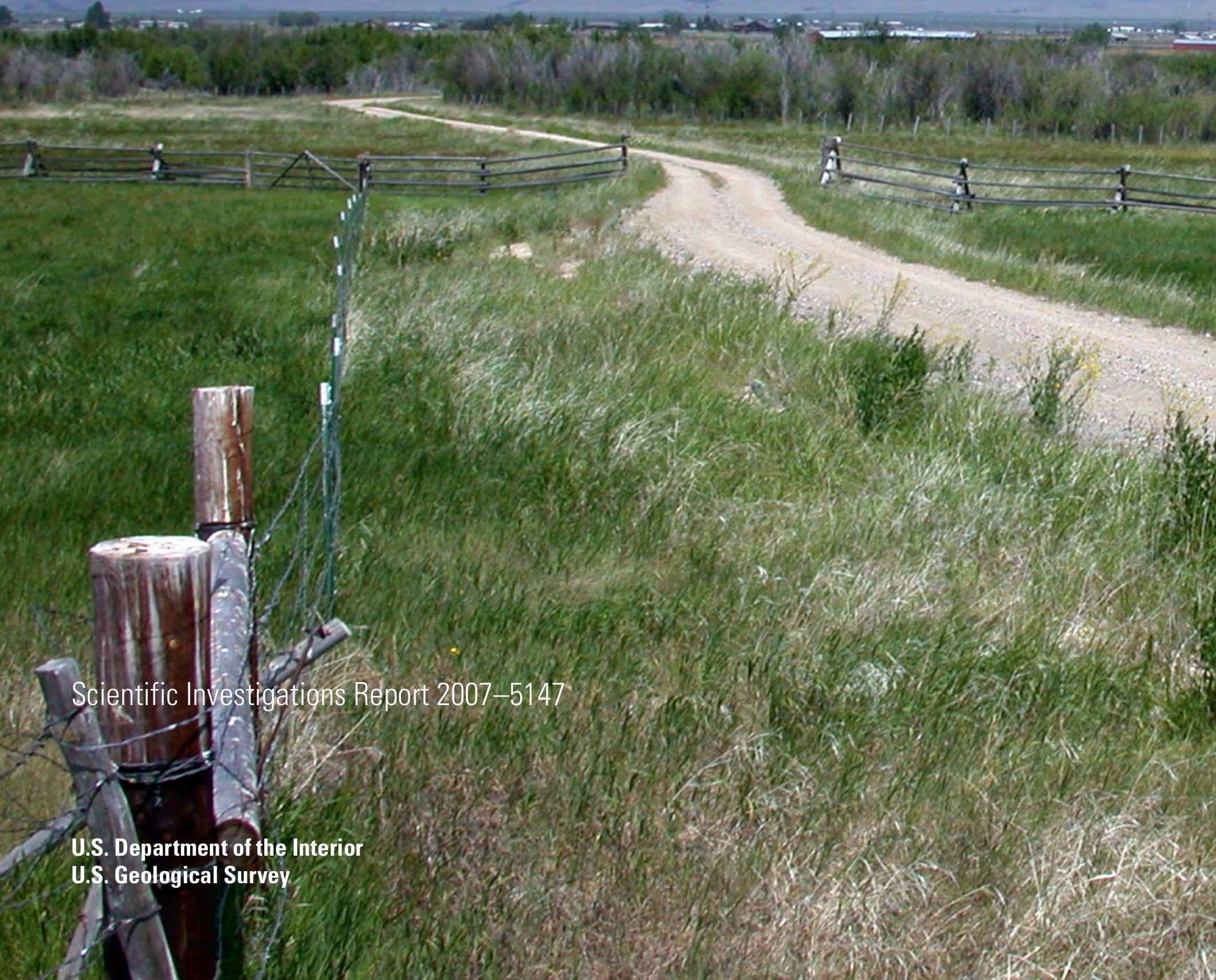


Cover. Grant-Kohrs Ranch National Historic Site, Montana, by W. Schweiger, National Park Service. 


\section{Assessment of Historical Water-Quality Data for National Park Units in the Rocky Mountain Network, Colorado and Montana, through 2004}

By M. Alisa Mast

Prepared in cooperation with the National Park Service

Scientific Investigations Report 2007-5147 


\section{U.S. Department of the Interior DIRK KEMPTHORNE, Secretary}

\section{U.S. Geological Survey \\ Mark D. Myers, Director}

\section{U.S. Geological Survey, Reston, Virginia: 2007}

For product and ordering information:

World Wide Web: http://www.usgs.gov/pubprod

Telephone: 1-888-ASK-USGS

For more information on the USGS--the Federal source for science about the Earth, its natural and living resources, natural hazards, and the environment:

World Wide Web: http://www.usgs.gov

Telephone: 1-888-ASK-USGS

Any use of trade, product, or firm names is for descriptive purposes only and does not imply endorsement by the U.S. Government.

Although this report is in the public domain, permission must be secured from the individual copyright owners to reproduce any copyrighted materials contained within this report.

Suggested citation:

Mast, M.A., 2007, Assessment of historical water-quality data for National Park units in the Rocky Mountain Network, Colorado and Montana, through 2004: U.S. Geological Survey Scientific Investigations Report 2007-5147, 79 p. 


\section{Contents}

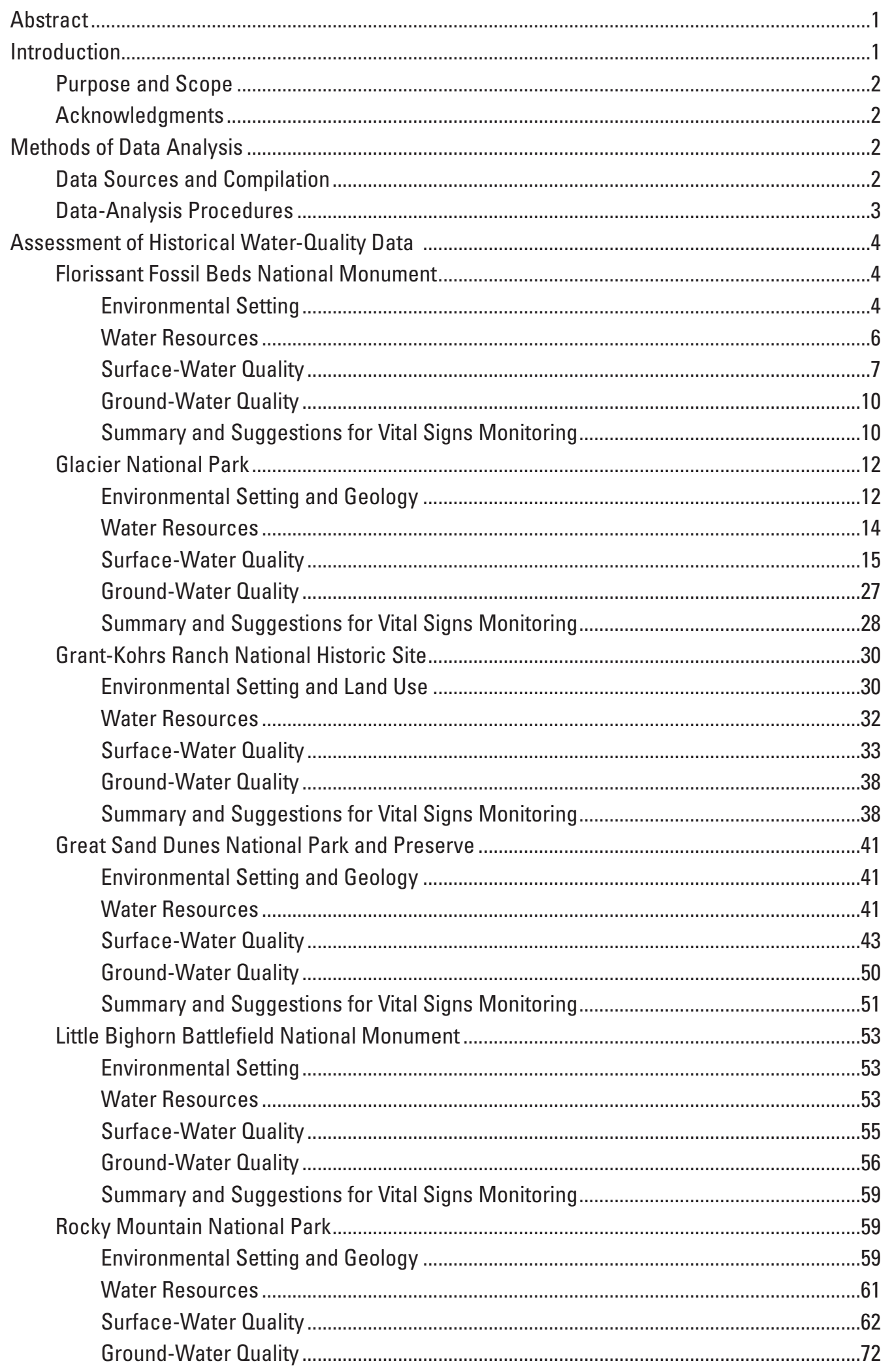


Summary and Suggestions for Vital Signs Monitoring ...................................................73

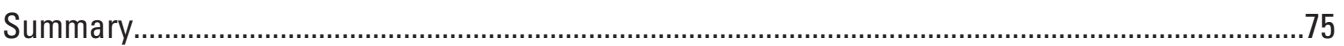

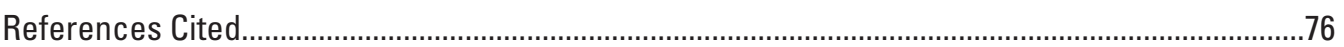

\section{Figures}

1-2. Maps showing-

1. Location of the six National Park units in the Rocky Mountain Network, Colorado and Montana

2. Location of water-quality sampling sites near Florissant Fossils Beds National Monument, Colorado

3-4. Graphs showing -

3. Daily streamflow range at Fountain Creek at Green Mountain Falls (USGS streamflow-gaging station 07099990) near Florissant Fossils Beds National Monument, Colorado, 2001-2005

4. Seasonal variation in water quality at selected sites in Florissant Fossil Beds National Monument, Colorado

5. Map showing location of water-quality sampling sites in Glacier National Park, Montana .13

6-9. Graphs showing-

6. Daily streamflow range at Swiftcurrent Creek at Many Glacier (S81) in Glacier National Park, Montana, 1986-2005

7. Periodic water-level measurements at selected shallow ground-water wells in Glacier National Park, Montana

8. Seasonal variation in water quality at Swiftcurrent Creek above Swiftcurrent Lake at Many Glacier (S78) in Glacier National Park, Montana, 2001-2004

9. Spatial variation in surface-water quality at selected sites in Glacier National Park, Montana

10. Map showing location of water-quality sampling sites near Grant-Kohrs Ranch National Historic Site, Montana

11-14. Graphs showing-

11. Daily streamflow range at Clark Fork River at Deer Lodge (S3) near GrantKohrs Ranch National Historic Site, Montana, 1979-2005.

12. Ground-water level measurements at selected monitoring wells in GrantKohrs Ranch National Historic Site, Montana, 2000.

13. Seasonal variation in water quality at sites $\mathrm{S} 1$ and $\mathrm{S} 3$ on the Clark Fork River near Grant-Kohrs Ranch National Historic Site, Montana, 1982-2004...........36

14. Diurnal variations in $\mathrm{pH}$, manganese, and zinc at Clark Fork River (S6) in Grant-Kohrs Ranch National Historic Site, Montana, July 21-23, 1994.

15. Map showing location of streamflow-gaging stations and ground-water wells in Great Sand Dunes National Park and Preserve, Colorado

16. Graph showing daily streamflow range at Medano Creek (station MEDSANCO) in Great Sand Dunes National Park and Preserve, Colorado, 1992-2004...

17. Map showing location of surface-water sampling sites in Great Sand Dunes National Park and Preserve, Colorado.

18. Graph showing spatial variation in water quality at selected sites in Great Sand Dunes National Park and Preserve, Colorado. 
19. Graph showing seasonal variation in selected constituent concentrations at Medano Creek near Mosca (S14) in Great Sand Dunes National Park and Preserve, Colorado

20. Map showing location of water-quality sampling sites near Little Bighorn Battlefield National Monument, Montana.

21-23. Graphs showing-

21. Daily streamflow range at Little Bighorn River (station 453400107263001) in Little Bighorn National Battlefield Monument, Montana, 1999-2005.

22. Ground-water level measurements at site G3 in Little Bighorn National Battlefield Monument, Montana

23. Variation in water quality with depth at selected ground-water wells in Little Bighorn National Battlefield Monument, Montana, 1977

24. Map showing location of lake and pond sampling sites in Rocky Mountain National Park, Colorado

25-28. Graphs showing-

25. Daily streamflow range at Colorado River near Baker Gulch (USGS

streamflow-gaging station 09010500) in Rocky Mountain National Park, Colorado, 1986-2005

26. Seasonal variation in selected constituent concentrations in water samples from the surface and bottom of Sky Pond (S78) in Rocky Mountain National Park, Colorado, 1982-2004

27. Comparison of water-quality data for selected lakes on the east and west sides of the Continental Divide in Rocky Mountain National Park, Colorado

28. Temporal trends in annual precipitation at Loch Vale NADP station and calcium concentrations at The Loch (S33) in Rocky Mountain National Park, Colorado

\section{Tables}

1. Number of water-quality sites, samples, and results retrieved and compiled for park units in the Rocky Mountain Network, Colorado and Montana, through 2004..........3

2. Water-quality sampling sites near Florissant Fossil Beds National Monument, Colorado

3. Period of record and types of analyses conducted for surface-water samples collected near Florissant Fossil Beds National Monument, Colorado

4. Summary of selected water-quality data for surface-water sites in Florissant Fossil Beds National Monument, Colorado, 1976 to 1997

5. Summary of selected water-quality data for ground-water sites in Florissant Fossil Beds National Monument, Colorado

6. Surface-water sampling sites in Glacier National Park, excluding sites on the North and Middle Forks of the Flathead River, Montana

7. Period of record and types of analyses conducted for surface-water samples in Glacier National Park, excluding sites on the North and Middle Forks of the Flathead River, Montana

8. Water-quality sampling sites on the North Fork and Middle Fork of the Flathead River in Glacier National Park, Montana

9. Summary of selected water-quality data for surface-water sites in Glacier National Park excluding the Middle and North Forks of the Flathead River, Montana, 1980 to 2004 
10. Summary of selected water-quality data for sampling sites on the North Fork and Middle Fork of the Flathead River in Glacier National Park, Montana, 1980 to 2004 .

11. Results of the seasonal Kendall test for trends in streamflow and unadjusted and flow-adjusted constituent concentrations for selected surface-water stations in Glacier National Park, Montana

12. Ground-water sampling sites in Glacier National Park, Montana .................................28

13. Summary of selected water-quality data for ground-water sites in Glacier National Park, Montana, 1972 to 1981

14. Surface-water sampling sites near Grant-Kohrs Ranch Ranch National Historic Site, Montana

15. Period of record and types of analyses conducted on surface-water samples collected along the main stem of the Clark Fork River near Grant-Kohrs Ranch National Historic Site, Montana

16. Summary of selected water-quality data for main-stem sites on the Clark Fork River near Grant-Kohrs Ranch National Historic Site, Montana, 1980 to 2004

17. Results of the seasonal Kendall test for trends in streamflow and unadjusted and flow-adjusted constituent concentrations for two stations on the Clark Fork River near Grant-Kohrs Ranch National Historic Site, Montana

18. Ground-water sampling sites near Grant-Kohrs Ranch National Historic Site, Montana

19. Summary of selected water-quality data for ground-water sites in Grant-Kohrs Ranch National Historic Site, Montana, 1980 to 2000

20. Surface-water sampling sites in Great Sand Dunes National Park and Preserve, Colorado

21. Period of record and types of analyses conducted on surface-water samples collected in Great Sand Dunes National Park and Preserve, Colorado.

22. Summary of selected water-quality data for surface-water sites in Great Sand Dunes National Park and Preserve, Colorado, 1964 to 2001.

23. Ground-water sampling sites in Great Sand Dunes National Park and Preserve, Colorado

24. Summary of selected water-quality data for ground-water sites in Great Sand Dunes National Park and Preserve, 1946 to 2000.

25. Water-quality sampling sites near Little Bighorn Battlefield National Monument, Montana

26. Summary of selected water-quality data for the Little Bighorn River adjacent to Little Bighorn Battlefield National Monument, Montana.

27. Summary of selected water-quality data for ground-water sites in the Little Bighorn Battlefield National Monument, Montana.

28. Lake and pond sampling sites in Rocky Mountain National Park, Colorado

29. Period of record and types of analyses conducted at lake and pond sampling sites in Rocky Mountain National Park, Colorado.

30. Summary of selected water-quality data for lake and pond sampling sites in Rocky Mountain National Park, Colorado, 1981 to 2004.

31. Results of the seasonal Kendall test for trends in streamflow and unadjusted and flow-adjusted constituent concentrations for Rocky Mountain National Park, Colorado

32. Ground-water sampling sites in Rocky Mountain National Park, Colorado .73

33. Summary of selected water-quality data for ground-water sites in Rocky Mountain National Park, Colorado, 1971 to 2003 


\section{Conversion Factors, Abbreviations, and Datums}

\begin{tabular}{|c|c|c|}
\hline Multiply & By & To obtain \\
\hline \multicolumn{3}{|c|}{ Length } \\
\hline centimeter $(\mathrm{cm})$ & 0.3937 & inch (in.) \\
\hline meter $(\mathrm{m})$ & 3.281 & foot $(\mathrm{ft})$ \\
\hline meter $(\mathrm{m})$ & 1.094 & yard (yd) \\
\hline kilometer (km) & 0.6214 & mile (mi) \\
\hline \multicolumn{3}{|c|}{ Area } \\
\hline square meter $\left(\mathrm{m}^{2}\right)$ & 0.0002471 & acre \\
\hline hectare (ha) & 2.471 & acre \\
\hline square kilometer $\left(\mathrm{km}^{2}\right)$ & 247.1 & acre \\
\hline square meter $\left(\mathrm{m}^{2}\right)$ & 10.76 & square foot $\left(\mathrm{ft}^{2}\right)$ \\
\hline hectare (ha) & 0.003861 & square mile $\left(\mathrm{mi}^{2}\right)$ \\
\hline square kilometer $\left(\mathrm{km}^{2}\right)$ & 0.3861 & square mile $\left(\mathrm{mi}^{2}\right)$ \\
\hline \multicolumn{3}{|c|}{ Volume } \\
\hline liter $(\mathrm{L})$ & 33.82 & ounce, fluid (fl. oz) \\
\hline liter $(\mathrm{L})$ & 2.113 & pint (pt) \\
\hline liter $(\mathrm{L})$ & 1.057 & quart (qt) \\
\hline liter $(\mathrm{L})$ & 0.2642 & gallon (gal) \\
\hline cubic meter $\left(\mathrm{m}^{3}\right)$ & 264.2 & gallon (gal) \\
\hline cubic meter $\left(\mathrm{m}^{3}\right)$ & 0.0002642 & million gallons (Mgal) \\
\hline liter $(\mathrm{L})$ & 61.02 & cubic inch $\left(\mathrm{in}^{3}\right)$ \\
\hline cubic meter $\left(\mathrm{m}^{3}\right)$ & 35.31 & cubic foot $\left(\mathrm{ft}^{3}\right)$ \\
\hline cubic meter $\left(\mathrm{m}^{3}\right)$ & 1.308 & cubic yard $\left(\mathrm{yd}^{3}\right)$ \\
\hline cubic meter $\left(\mathrm{m}^{3}\right)$ & 0.0008107 & acre-foot (acre-ft) \\
\hline \multicolumn{3}{|c|}{ Flow rate } \\
\hline cubic meter per second $\left(\mathrm{m}^{3} / \mathrm{s}\right)$ & 70.07 & acre-foot per day (acre-ft/d) \\
\hline cubic meter per second $\left(\mathrm{m}^{3} / \mathrm{s}\right)$ & 35.31 & cubic foot per second $\left(\mathrm{ft}^{3} / \mathrm{s}\right)$ \\
\hline liter per second (L/s) & 15.85 & gallon per minute (gal/min) \\
\hline \multicolumn{3}{|c|}{ Mass } \\
\hline $\operatorname{gram}(\mathrm{g})$ & 0.03527 & ounce, avoirdupois (oz) \\
\hline kilogram (kg) & 2.205 & pound avoirdupois (lb) \\
\hline
\end{tabular}

Temperature in degrees Celsius $\left({ }^{\circ} \mathrm{C}\right)$ may be converted to degrees Fahrenheit $\left({ }^{\circ} \mathrm{F}\right)$ by ${ }^{\circ} \mathrm{F}=\left(1.8 x^{\circ} \mathrm{C}\right)+32$.

Vertical coordinate information is referenced to the North American Vertical Datum of 1988 (NAVD 88).

Horizontal coordinate information is referenced to the North American Datum of 1983 (NAD 83). Altitude, as used in this report, refers to distance above the vertical datum.

Water year, as used in this report, refers to the period October 1 through September 30 and is designated by the year in which it ends. 


\section{Abbreviations, acronyms, and symbols used in this report}

\begin{tabular}{|c|c|}
\hline CASNET & Clean Air Status and Trends \\
\hline CDPHE & Colorado Department of Public Health and Environment \\
\hline ESTREND & Estimate Trend \\
\hline FLBS & Flathead Lake Biological Station \\
\hline FLFO & Florissant Fossil Beds National Monument \\
\hline GLAC & Glacier National Park \\
\hline GRKO & Grant-Kohrs Ranch National Historic Site \\
\hline GRSA & Great Sand Dunes National Park and Preserve \\
\hline I\&M & Inventory and Monitoring Program \\
\hline LIBI & Little Bighorn Battlefield National Monument \\
\hline LVWS & Loch Vale Watershed \\
\hline MTDEO & Montana Department of Environmental Quality \\
\hline NADP & National Atmospheric Deposition Program \\
\hline NPS & National Park Service \\
\hline NURE & National Uranium Resource Evaluation \\
\hline NWIS & National Water Information System \\
\hline ROMN & Rocky Mountain Network \\
\hline ROMO & Rocky Mountain National Park \\
\hline STORET & Storage and Retrieval System \\
\hline TVS & Table Value Standard \\
\hline USEPA & U.S. Environmental Protection Agency \\
\hline USGS & U.S. Geological Survey \\
\hline VNRP & Voluntary Nutrient Reduction Program \\
\hline $\mathrm{cm}$ & centimeter \\
\hline $\mathrm{col} / 100 \mathrm{~mL}$ & colonies per 100 milliliters \\
\hline $\mathrm{ft}^{3} / \mathrm{s}$ & cubic feet per second \\
\hline $\mathrm{ft}^{3} / \mathrm{s} / \mathrm{yr}$ & cubic feet per second per year \\
\hline $\mathrm{kg} \mathrm{N} / \mathrm{ha} / \mathrm{yr}$ & kilograms of nitrogen per hectare per year \\
\hline $\mathrm{km}$ & kilometer \\
\hline $\mathrm{km}^{2}$ & square kilometers \\
\hline $\mathrm{L} / \mathrm{min}$ & liters per minute \\
\hline $\mathrm{L} / \mathrm{s}$ & liters per second \\
\hline
\end{tabular}




$\begin{array}{ll}\mathrm{m} & \text { meter } \\ \mathrm{m} / \mathrm{km} & \text { meters per kilometer } \\ \mathrm{mg} / \mathrm{L} & \text { milligrams per liter } \\ \mathrm{mg} / \mathrm{L} / \mathrm{yr} & \text { milligrams per liter per year } \\ \mu \mathrm{eq} / \mathrm{L} / \mathrm{yr} & \text { microequivalents per liter per year } \\ \mu \mathrm{g} / \mathrm{L} & \text { micrograms per liter } \\ \mu \mathrm{S} / \mathrm{cm} & \text { microsiemens per centimeter } \\ { }^{\circ} \mathrm{C} & \text { degrees Celsius } \\ < & \text { less than } \\ > & \text { greater than } \\ \leq & \text { less than or equal to }\end{array}$





\title{
Assessment of Historical Water-Quality Data for National Park Units in the Rocky Mountain Network, Colorado and Montana, through 2004
}

\author{
By M. Alisa Mast
}

\section{Abstract}

This report summarizes historical water-quality data for six National Park units that compose the Rocky Mountain Network. The park units in Colorado are Florissant Fossil Beds National Monument, Great Sand Dunes National Park and Preserve, and Rocky Mountain National Park; and in Montana, they are Glacier National Park, Grant-Kohrs Ranch National Historic Site, and Little Bighorn Battlefield National Monument. This study was conducted in cooperation with the Inventory and Monitoring Program of the National Park Service to aid in the design of an effective and efficient waterquality monitoring plan for each park. Data were retrieved from a number of sources for the period of record through 2004 and compiled into a relational database. Descriptions of the environmental setting of each park and an overview of the park's water resources are presented. Statistical summaries of waterquality constituents are presented and compared to aquatic-life and drinking-water standards. Spatial, seasonal, and temporal patterns in constituent concentrations also are described and suggestions for future water-quality monitoring are provided.

\section{Introduction}

In 1992, the National Park Service (NPS) initiated the Inventory and Monitoring (I\&M) program to help develop a stronger scientific basis for stewardship and management of natural resources across the National Park system (http:// science.nature.nps.gov/im/index.cfm, accessed November 2006). A primary goal of $I \& M$ is to complete basic inventories in parks with extensive natural resources and to provide resource managers with scientifically sound information on their status and trends in a readily accessible form. Another I\&M goal is to design and implement a program to monitor the 'vital signs' of park resources (National Park Service, 2005). Vital signs are selected physical, chemical, and biological elements and processes of park ecosystems that represent the overall health or condition of the park. Vital signs can be used as early warning signals to detect changes that could impair the long-term health of natural systems. Early detection of potential problems allows park managers to take steps to restore ecological health of park resources before serious damage occurs (National Park Service, 2005). To achieve these goals, the I\&M program organized the 270 parks in the National Park system into 32 networks, which link parks that share similar geographic and natural-resource characteristics. Parks within a network share funding and staff to improve efficiency and reduce costs and partner with universities and State and Federal agencies to assist with basic park resource inventories and monitoring.

One of the 32 networks is the Rocky Mountain Network (ROMN), which consists of six parks located along a northwest-southeast axis that follows the Continental Divide in the central Rocky Mountains (fig. 1). The six parks are Florissant Fossil Beds National Monument, Great Sand Dunes National Park and Preserve, and Rocky Mountain National Park in Colorado; and Glacier National Park, Grant-Kohrs Ranch National Historic Site, and Little Bighorn Battlefield National Monument in Montana. Twelve high-priority vital signs have been selected for monitoring in the six ROMN parks (http://wwwl.nature.nps.gov/im/units/romn/vital_signs. $c f m$, accessed April 2007). The vital signs include (1) wet and dry deposition, (2) weather and climate, (3) water chemistry, (4) surface-water dynamics, (5) freshwater communities, (6) invasive/exotic aquatic biota, (7) ground-water dynamics, (8) wetland communities, (9) invasive/exotic plants, (10) vegetation composition, structure, and soils, (11) focal species, and (12) landscape dynamics. One-half of the identified vital signs are related to aquatic resources because they are central to the park missions, and their protection is given high priority in each park's resource management plan, particularly in Glacier National Park, Great Sand Dunes National Park and Preserve, and Rocky Mountain National Park. Rocky Mountain National Park, for example, has 156 high-elevation lakes and more than 700 kilometers $(\mathrm{km})$ of streams in Class I wilderness (Britten and others, 2006), which provide habitat for numerous aquatic plants and animals, including the Federally listed greenback cutthroat trout and boreal toad. 


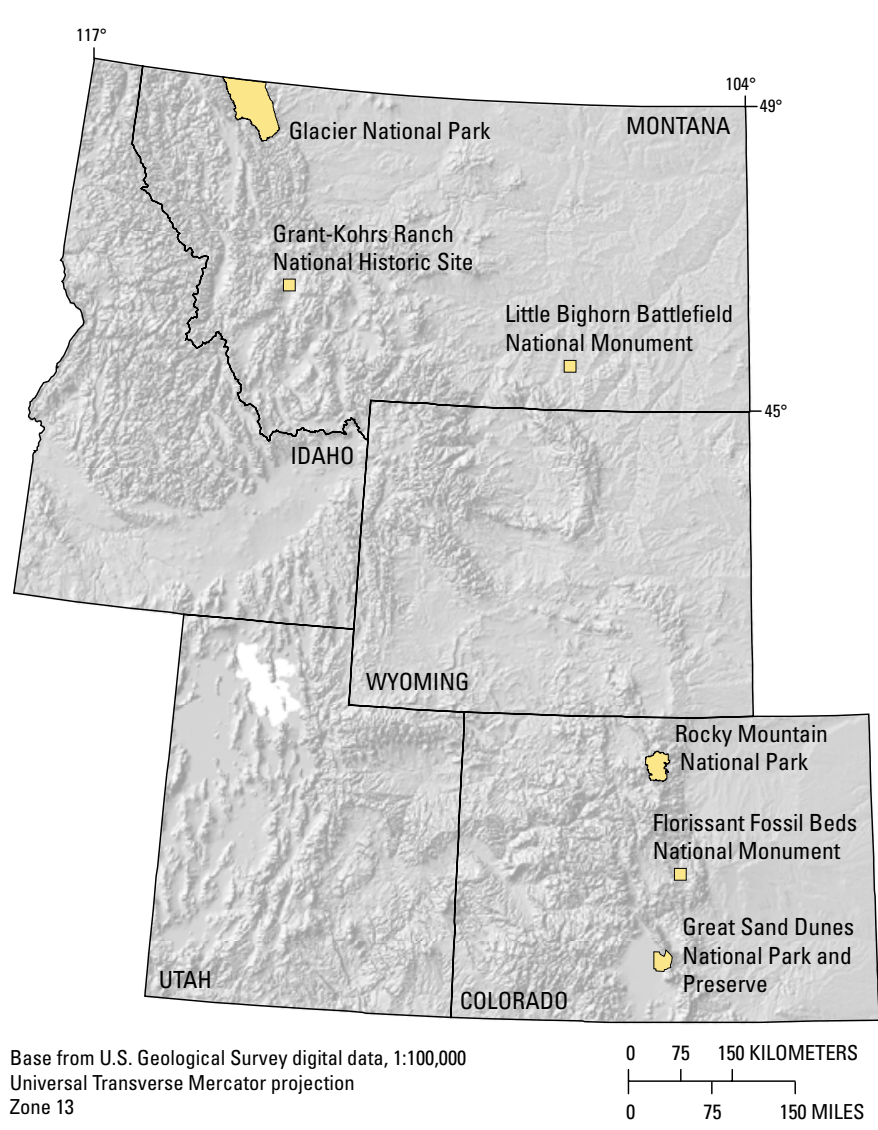

Figure 1. Location of the six National Park units in the Rocky Mountain Network, Colorado and Montana.

The ROMN staff currently (2007) is in the process of establishing protocols and developing an integrated monitoring plan for aquatic vital signs (including water quality), which is scheduled for implementation during 2009 to 2010. The general goal of the monitoring plan is to characterize the current status of and determine trends in the ecological condition of aquatic resources. Trend information is essential to assess the effectiveness of management and restoration activities and to provide early warning of environmental change. Water-quality monitoring also must be linked to Federal and State regulatory frameworks established under the 1977 Clean Water Act. As one of the first steps in the planning process, the U.S. Geological Survey (USGS), in cooperation with the NPS, conducted a study to compile, review, and summarize historical waterquality data for each park unit in the network. The main objective of this study was to provide a framework of information to aid in the design of an effective and efficient water-quality monitoring program for each of the six ROMN parks.

\section{Purpose and Scope}

The purpose of this report is to present summaries and hydrologic interpretations of water-quality data collected at surface- and ground-water sites in the six park units in the
ROMN. This report uses available water-quality data for the period of record through water year 2004 and focuses on field properties, major constituents, nutrients, and dissolved trace elements. The majority of the data were obtained from the USGS National Water Information System (NWIS) and the U.S. Environmental Protection Agency (USEPA) Storage and Retrieval System (STORET) databases. Descriptions of the environmental setting of each park and an overview of the park's water resources are provided. Statistical summaries of water-quality constituents are presented and compared to aquatic-life and drinking-water standards. Spatial, seasonal, and temporal patterns in constituent concentrations also are described and suggestions are made for design of a waterquality monitoring plan in each park. Hydrologic interpretations are considered preliminary because data sets generally are small and data were collected periodically for different study objectives. Other limitations to interpreting data from different sources include use of different analytical laboratories and methods, differences in laboratory reporting levels, and differences in field and laboratory quality-assurance procedures.

\section{Acknowledgments}

The author thanks Mike Britten, Brent Frakes, and E. William Schweiger of the NPS I\&M Program for their support of this project. Technical reviews of the report were provided by Julianne Brown of the USGS and E. William Schweiger of the NPS.

\section{Methods of Data Analysis}

Water-quality data were retrieved in electronic format from a number of difference sources, compiled into a relational database, and checked for general accuracy. Results for selected surface- and ground-water sites from each park unit were analyzed by statistical and graphical methods for hydrologic interpretation.

\section{Data Sources and Compilation}

Most of the water-quality data analyzed in this report were retrieved from the NWIS (http://waterdata.usgs.gov/ nwis, accessed August 2007) and STORET (http://www.epa. gov/storet/dbtop.html, accessed August 2007) databases for the period of record through 2004. Additional datasets were obtained from several USGS and NPS researchers and graduate students provided they were available in electronic format. Study areas were defined for each park unit based on the park boundary, a buffer area (up to $1 \mathrm{~km}$ ) around the boundary, and headwater areas of streams flowing through but not originating in the park (see Grape Creek drainage in fig. 2 for example). All water-quality data for surface- and ground-water sites 
located within the defined study areas were retrieved from the different data sources and compiled into a relational database using the Access software, herein referred to as the "ROMN database." In the database, data are stored in three core tables that include a site attribute table, a sampling event table, and a water-quality results table. Additional related information is stored in a number of lookup tables and ancillary tables. Data in the results table are stored using 5-character numeric codes called parameter codes that define a specific type of data (http://nwis.waterdata.usgs.gov/usa/nwis/pmcodes, accessed November 2006). For example, the parameter code for field $\mathrm{pH}$ in unfiltered water is 00400 and for laboratory $\mathrm{pH}$ in unfiltered water is 00403 . Parameter codes were grouped into 18 waterquality categories, such as field properties, nutrients, and major constituents, to facilitate retrieval of information from the database. All data were screened for outliers and checked against expected ranges for constituent concentrations (National Park Service, 1998); suspect values were moved to a separate table in the database. All USGS trace-element data were retained in the database although it is documented that dissolved concentrations in samples collected before 1992 may have been contaminated during sample collection and processing (USGS Office of Water Quality Technical Memorandum 91.10, available at http://water.usgs.gov/admin/memo/QW/index. html, accessed November 2006). Because field and laboratory quality-assurance data are not available for the majority of the retrieved records, a more comprehensive review of data quality was not possible.

The final ROMN database contained water-quality data collected at 1,696 surface-water sites and 240 ground-water sites from 1941 to 2004 (table 1). For these 1,936 sites, there were a total of 35,534 samples with more than 400,000 individual results most of which were field properties (33 percent), and major-constituent (28 percent), nutrient (12 percent), and trace-element (13 percent) analyses. The number of sites, samples, and results in the ROMN database by park unit and location relative to the park boundary are shown in table 1 . More than 80 percent of the retrieved results were for Glacier National Park and Rocky Mountain National Park, which are the largest parks in the ROMN network.

\section{Data-Analysis Procedures}

For this report, a subset of water-quality properties and constituents from the ROMN database were used for data analysis; primarily field properties, major constituents, nutrients, and trace elements. In addition, only data for sites located within or immediately adjacent to the park unit boundaries were selected. In some cases, results for the same constituent are stored under different parameter codes because of differences in analytical methods, sampling techniques, reporting units, or data sources. For these constituents, the results were aggregated into a single parameter to obtain a more complete and continuous record for data analysis. For example, $\mathrm{pH}$ often is reported under three different parameter codes; field $\mathrm{pH}$ (P0400), laboratory $\mathrm{pH}(00403)$, and $\mathrm{pH}$ in standard units (00406). In this case, results for parameter codes 00403 and 00406 were combined with parameter code 00400 for samples where field $\mathrm{pH}$ was not reported. Nutrients also are commonly reported under multiple parameter codes. Guidelines used for aggregating common nutrient species are described by Mueller and others (1995). Total dissolved solids were computed for samples with complete major constituent analyses by summing the concentrations of the individual constituents. For this report, 'dissolved' constituents refer to concentrations measured in filtered samples and 'total' constituents refer to concentrations measured in unfiltered samples. Based on the range of $\mathrm{pH}$ in surface and ground water samples from the parks, the bicarbonate anion was assumed to be the dominant form of alkalinity.

Descriptive summary statistics for surface-water and ground-water quality were computed and tabulated and compared to aquatic-life standards and drinking-water regulations. Because data were compiled from different sources and periods of record, the datasets often contained more than one laboratory reporting level for a given constituent, particularly for nutrients and trace elements. The original censored values including 'zero' values were retained in the database, which sometimes resulted in minimum values less than minimum censored values in the water-quality summary tables. For the three parks in Colorado, water-quality standards and clas-

Table 1. Number of water-quality sites, samples, and results retrieved and compiled for park units in the Rocky Mountain Network, Colorado and Montana, through 2004.

[Colo., Colorado; Mont., Montana; first number is number of sites, samples or results inside park boundary; number in parentheses is number of sites, samples or results outside park boundary]

\begin{tabular}{|c|c|c|c|c|c|c|}
\hline \multirow[b]{2}{*}{ Park unit } & \multicolumn{3}{|c|}{ Surface water } & \multicolumn{3}{|c|}{ Ground water } \\
\hline & Sites & Samples & Results & Sites & Samples & Results \\
\hline Florissant Fossil Beds National Monument, Colo. & $8(5)$ & $43(17)$ & $476(198)$ & $2(0)$ & $2(0)$ & $88(0)$ \\
\hline Rocky Mountain National Park, Colo. & $548(120)$ & $12,796(7,731)$ & $144,814(94,399)$ & $45(18)$ & $346(20)$ & $4,573(1,022)$ \\
\hline Glacier National Park, Mont. & $527(273)$ & $3,147(7,767)$ & $58,623(48,956)$ & $22(0)$ & $74(0)$ & $2,480(0)$ \\
\hline Little Bighorn Battlefield National Monument, Mont. & $2(30)$ & $5(1,284)$ & $253(15,806)$ & $3(72)$ & $6(80)$ & $202(2,161)$ \\
\hline
\end{tabular}


sifications were obtained from the Colorado Department of Public Health and Environment (CDPHE) (http://www.cdphe. state.co.us/regulations/wqccregs/, accessed November 2006). For the three parks in Montana, water-quality standards and classifications were obtained from the Montana Department of Environmental Quality (MTDEQ) (http://www.deq.state. mt.us/wqinfo/Standards/Index.asp, accessed November 2006). Some trace elements have table value standards (TVS), which are site-specific standards based on stream hardness. For these constituents, the standard was calculated based on the median hardness for the water bodies of interest. Both chronic and acute standards typically are established for trace elements. Chronic standards, which usually are lower than the acute standards, were used for comparison with trace-element concentrations in surface water.

Temporal trends in water-quality constituent concentrations were tested using the Estimate Trend (ESTREND) computer program (Schertz and others, 1991), which uses the nonparametric seasonal Kendall test or the Tobit procedure if more than 5 percent of the data are censored. Temporal trends were tested only on sites with less than 50 percent censored data, and with at least 5 years of quarterly data. If discharge data were available, concentrations were adjusted for flow-related variability, which not only improves the power of the statistical test, but decreases the possibility that the observed trends were an artifact of the sampling discharge record (Hirsch and others, 1982; Schertz and others, 1991). Flow adjustment was not made if the concentration-discharge model was not significant at the 90 percent confidence level (Schertz and others, 1991) or if more than 5 percent of the data were censored. Trends were calculated using unadjusted and flow-adjusted concentrations and were considered statistically significant at the 99 percent confidence level (p-value $\leq 0.01$ ). The ESTREND procedure also computes a trend slope, which represents the median rate of change in concentration or discharge for the selected period of record.

\section{Assessment of Historical Water- Quality Data}

Historical data were assessed to describe water-quality conditions for the six park units in the ROMN. Results for each of the parks are discussed separately in the following sections of this report. Each section provides a brief description of the environmental setting of the park and an overview of the park's water resources. Statistical summaries of selected water-quality constituents are presented and compared to aquatic-life and drinking-water standards. Spatial, seasonal, and temporal patterns in constituent concentrations were examined to help identify natural and anthropogenic factors controlling water quality in each of the six parks. Suggestions are provided for consideration in designing water-quality monitoring plans.
The discussion of water quality in this report primarily focuses on field properties, major constituents, nutrients, and dissolved trace elements. The most commonly measured field properties are water temperature, dissolved oxygen, $\mathrm{pH}$, and specific conductance, which are referred to as "core field properties" by the NPS. Water temperature is important because it controls the rate of chemical reactions, which in turn affects biological activity in aquatic environments. Dissolved oxygen is necessary for the survival of many aquatic organisms, and $\mathrm{pH}$ is important because it determines the solubility and biological availability of chemical constituents such as nutrients and trace elements. Specific conductance is proportional to the concentration of major dissolved constituents, the most common of which are bicarbonate, calcium, chloride, fluoride, magnesium, potassium, silica, sodium, and sulfate. The sum of the concentrations of these dissolved constituents is referred to as dissolved solids concentration. The primary source of major dissolved constituents in surface and ground water is weathering of minerals in soil, bedrock, and unconsolidated material. Atmospheric deposition can be a substantial source of dissolved chloride and sulfate particularly in areas with crystalline bedrock, which typically has low chlorine and sulfur contents. Human activities also can affect major-constituent concentrations; for example, mine wastes are a source of sulfate, and sewage effluent and road salt can be sources of sodium and chloride. Major nutrients are compounds of nitrogen and phosphorus that are needed for plant growth. Excess nutrients in surface water can cause overgrowth of algae, which can lead to degradation of aquatic habitat. Sewage effluent, livestock waste, and fertilizer typically are the largest sources of nutrients to surface and ground water. In areas with minimal human activities, such as National Parks, atmospheric deposition of nitrogen derived from fossil fuel combustion and agriculture can be an important source of nutrients to lakes and streams. Trace elements generally are associated with mining and urban activities, and are important indicators of water quality because elevated concentrations can be toxic to aquatic life.

\section{Florissant Fossil Beds National Monument}

\section{Environmental Setting}

Florissant Fossil Beds National Monument (FLFO) is located on the eastern slope of the Colorado Front Range approximately $48 \mathrm{~km}$ west of Colorado Springs, Colorado (fig. 2). The park covers 24.2 square kilometers $\left(\mathrm{km}^{2}\right)$, ranges in elevation from 2,500 to 2,700 meters (m), and is characterized by an open mountain valley surrounded by rounded rocky hills. Vegetation is dominated by Arizona Fescue and Mountain Muly herbaceous cover along the valley floor and ponderosa pine woodland and Colorado blue spruce/Douglas fir forest on the surrounding hills (Britten and others, 2006). Climate is characterized by cool winters with warm summers. Mean monthly air temperatures near the park headquarters 


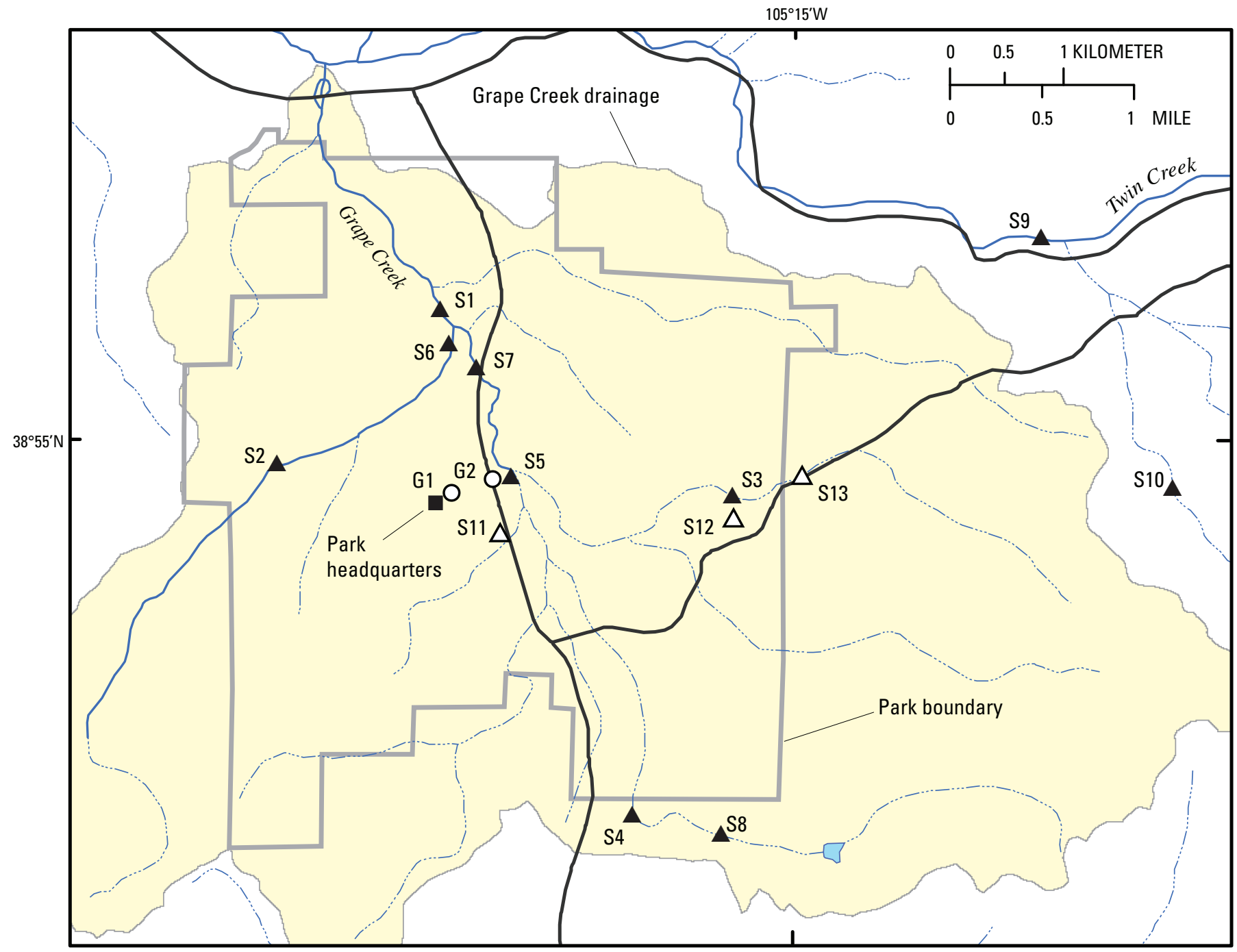

Base from U.S. Geological Survey digital data, 1:100,000

Universal Transverse Mercator projection

Zone 13

\section{EXPLANATION}
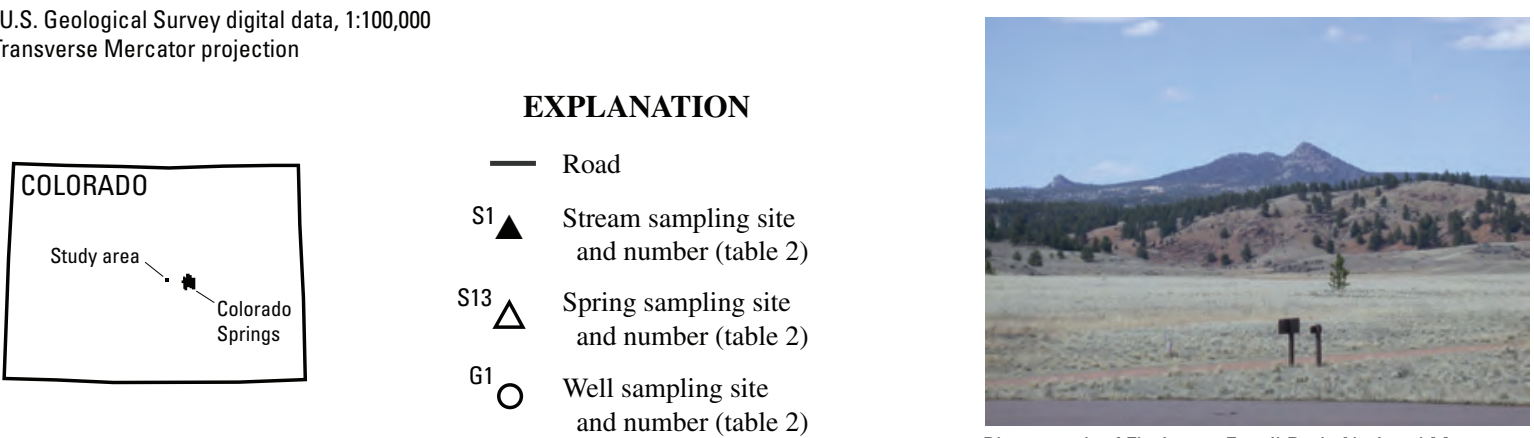

Photograph of Florissant Fossil Beds National Monument by W. Schweiger, National Park Service

Figure 2. Location of water-quality sampling sites near Florissant Fossils Beds National Monument, Colorado. 
range from $-5.3{ }^{\circ} \mathrm{C}$ in January to $15.3{ }^{\circ} \mathrm{C}$ in July (http://www. wrcc.dri.edu/summary/climsmco.html, accessed May 2006). Annual average precipitation is 38 centimeters (cm) most of which falls during afternoon thundershowers between May and September. The area receives about $145 \mathrm{~cm}$ of snowfall each year, mostly between October and April.

Bedrock is the Pikes Peak Granite, which is a pink to reddish-tan coarse-grained granite and quartz monzonite that forms hills and ridges in the monument (Wobus and Epis, 1978). Along the valley floor, the granite is overlain by the Eocene Florissant Formation, which in most places is mantled by a thin layer of Quaternary alluvium and colluvium. The Florissant Formation is composed of andesitic tuff and volcanic mudflow breccia interlayered with tuffaceous shale and mudstone that were deposited in an ancient lake. These lake sediments contain abundant plant, insect, and tree fossils for which FLFO is renowned (http://www2.nature.nps.gov/ geology/parks/flfo/, accessed May 2006).

Land-use activities in FLFO are related to visitor use and park administration including the visitor center and park headquarters, $9.7 \mathrm{~km}$ of roads, and two picnic areas. Land adjacent to the monument is privately owned and is used as rangeland. According to the park's resource management plan, rapid expansion of subdivisions adjacent to the monument and anticipated increases in visitor use pose the largest threats to natural resources in the park (National Park Service, 2006). In the1930s and 1940s, ranchers and farmers constructed 44 earthen dams in park drainages, which were intended to provide erosion control and facilitate water retention and diversion for agriculture. By 2001, removal of five dams was completed to restore the drainages to their natural condition; however, there are no immediate plans to remove the remaining dams (National Park Service, 2006). Surface water in the monument is not used for irrigation, and drinking water is obtained from a ground-water well located near the park headquarters (G1, fig. 2 ). The well is $50 \mathrm{~m}$ deep and capable of pumping an estimated 15 to 23 liters per minute (L/min) (National Park Service, 2006). The ground water has high dissolved solid concentrations and must be treated to make it potable. The park also has water rights for a second well (G2, fig. 2), which is $18 \mathrm{~m}$ deep and capable of pumping 2 to $4 \mathrm{~L} / \mathrm{min}$ and is used for sanitation purposes only (National Park Service, 2006). Water supply in the park is monitored for health-based contaminants according to the Colorado Drinking Water Program (http://www.cdphe. state.co.us/wq/drinkingwater/index.html, accessed August 2007). Wastewater in FLFO is handled by a septic system located near the park headquarters.

\section{Water Resources}

The main drainage in FLFO is Grape Creek (fig. 2), which flows north into Twin Creek, a tributary of the South Platte River. The lower reaches of Grape Creek are perennial, but its tributaries are intermittent. Flow characteristics of Grape Creek are not well characterized because the creek has not been gaged, nor have any streams of similar size in adjacent areas. The only available hydrologic data for Grape Creek are seven instantaneous measurements at site S1 (fig. 2) during the summer months (June to September), which ranged from 0.14 to 2.6 cubic feet per second $\left(\mathrm{ft}^{3} / \mathrm{s}\right)$, and three measurements at site $\mathrm{S} 4$, which ranged from 0.09 to $0.22 \mathrm{ft}^{3} / \mathrm{s}$. The closest streamflow-gaging station on a stream of similar size and elevation is Fountain Creek at Green Mountain Falls (USGS station 07099990), which is located $18 \mathrm{~km}$ east of FLFO. The Fountain Creek drainage ranges in elevation from 2,359 to 3,000 $\mathrm{m}$ and drains a $43-\mathrm{km}^{2}$ area. By comparison, Grape Creek (above the confluence with Twin Creek) ranges in elevation from 2,485 to 2,930 m, and drains an area of $55 \mathrm{~km}^{2}$. The range of daily streamflow values for Fountain Creek from 2001 to 2005 is shown in figure 3 . The smallest streamflows were observed during winter (November to February) and represent base flow from ground-water discharge. The largest streamflows generally are observed in late spring and early summer (April, May, and June) as a result of snowmelt runoff. Thundershowers contribute to streamflows through the summer months (July to September). Runoff during the snowmelt period (April, May, and June) at Fountain Creek accounts for more than 40 percent of the annual streamflow.

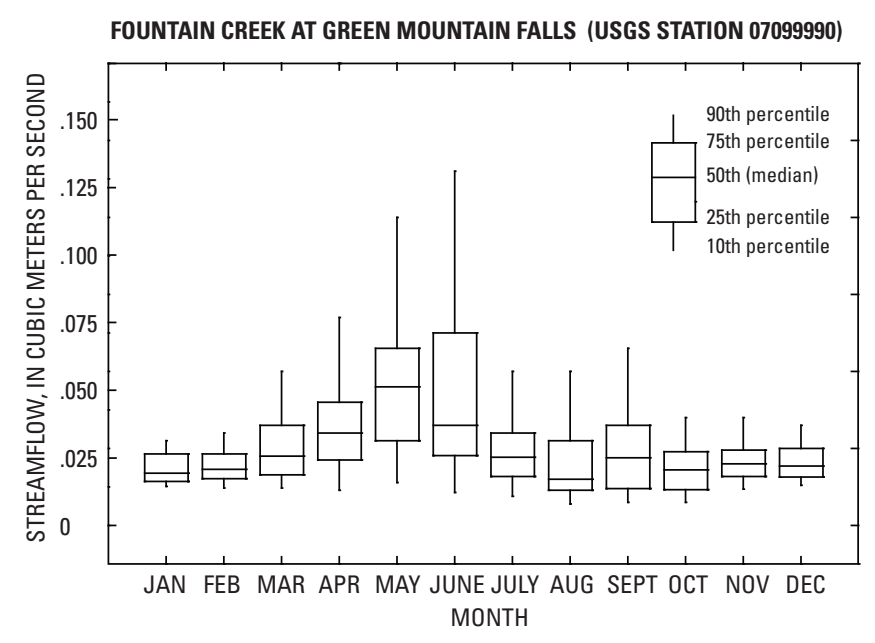

Figure 3. Daily streamflow range at Fountain Creek at Green Mountain Falls (USGS streamflow-gaging station 07099990) near Florissant Fossils Beds National Monument, Colorado, 2001-2005.

Although there are a number of ground-water fed seeps and springs in FLFO, the ground-water resources have not been studied and are poorly characterized. Because surficial deposits of alluvium and soils generally are thin in the foothills of the Front Range (Bossong and others, 2003), it is likely that aquifers in the granitic bedrock are the dominant ground-water resource in the park. Crystalline bedrock, such as granite, generally has low intergranular porosity, so open space that may contain water consists mostly of fractures and fracture networks (Bossong and others, 2003). 
Water-resource issues for FLFO include effects of park facilities and visitor use inside the park, and land-use activities such as grazing and residential development in the headwaters of Grape Creek (National Park Service, 2006). The waterquality constituents most likely to be affected by these activities include nutrients, sediment, and bacteria. Climate change may have long-term effects on streamflows and ground-water resources in the park.

\section{Surface-Water Quality}

Water-quality data are available for 13 surface-water sites in and adjacent to FLFO, including 10 streams and 3 springs (table 2, fig. 2). Sites S1-S4 were sampled by the NPS from 1995 to 1997 to characterize seasonal variation in core field properties (water temperature, specific conductance, dissolved oxygen, and $\mathrm{pH}$ ). Sites $\mathrm{S} 4$ and S5 were sampled in the early 1990s as part of the USEPA Environmental Monitoring and Assessment Program (http://www.epa.gov/emap/remap/index. html, accessed May 2006) and sites S6-S13 were sampled once each in 1976 as part of the National Uranium Resource Evaluation (NURE) program (http://pubs.usgs.gov/of/1997/ ofr-97-0492/, accessed May 2006). The ROMN database includes 60 water-quality samples collected at these 13 surface-water sites, most of which are field properties (39 percent), and major-constituent ( 23 percent), nutrient ( 8 percent), and trace-element (29 percent) analyses. The period of record and number of samples collected in each of these property or constituent categories are summarized in table 3 .
Water-quality data for the 13 surface-water sites in and adjacent to FLFO are summarized in table 4. Stream water in FLFO is a well buffered calcium-bicarbonate type; specific conductance ranged from 130 to 525 microsiemens per centimeter $(\mu \mathrm{S} / \mathrm{cm})$ and alkalinity ranged from 66 to 180 milligrams per liter $(\mathrm{mg} / \mathrm{L})$. The dominant cations in stream water were calcium (20 to $84 \mathrm{mg} / \mathrm{L}$ ) and sodium (7.6 to 17 $\mathrm{mg} / \mathrm{L}$ ), and the dominant anion was bicarbonate (based on $\mathrm{pH}$ and alkalinity). The predominance of these major dissolved constituents is attributed to weathering of carbonate minerals in the Florissant Formation and plagioclase in the Pikes Peak Granite. Other major anions were sulfate, which ranged from 6.7 to $73 \mathrm{mg} / \mathrm{L}$, and chloride, which ranged from 4.0 to 20 $\mathrm{mg} / \mathrm{L}$. Although these anions were less abundant than bicarbonate, concentrations were substantially larger than those measured at a nearby precipitation station (http://nadp.sws. uiuc.edu/, accessed May 2006) indicating they also are derived primarily from weathering of rocks and soils. Perhaps the most unusual result was the fluoride concentration measured at site $\mathrm{S} 5$, which ranged from 2.2 to $2.5 \mathrm{mg} / \mathrm{L}$. This concentration range is elevated compared to most natural waters, which typically are less than $1 \mathrm{mg} / \mathrm{L}$ (Hem, 1985). A potential source of fluoride to surface water in FLFO is the Pikes Peak Granite, which is enriched in fluoride (up to 0.5 weight percent) compared to most granitic rocks (Hawley and Wobus, 1977). Fluoride in the granite is present primarily in the minerals fluorite, topaz, biotite, and muscovite.

Nutrients measured in surface-water samples from FLFO include ammonia, nitrite, nitrate, orthophosphate, and total

Table 2. Water-quality sampling sites near Florissant Fossil Beds National Monument, Colorado.

[no., number; FLFO, Florissant Fossil Beds National Monument; ST, stream; SP, spring; GW, ground water; Identification numbers beginning with FLFO from USEPA STORET and identification numbers beginning with 3854 from USGS NWIS]

\begin{tabular}{|c|c|c|c|c|c|c|c|}
\hline $\begin{array}{l}\text { Site no. } \\
\text { (fig. 2) }\end{array}$ & Identification no. & Station name & Latitude & Longitude & Type & $\begin{array}{c}\text { No. of } \\
\text { samples }\end{array}$ & $\begin{array}{l}\text { Period of } \\
\text { record }\end{array}$ \\
\hline $\mathrm{S} 1$ & FLFO_NPS_GC2 & Grape Creek \#2 & $38^{\circ} 55^{\prime} 37^{\prime \prime}$ & $105^{\circ} 17^{\prime} 08^{\prime \prime}$ & ST & 12 & 1995-97 \\
\hline $\mathrm{S} 2$ & FLFO_NPS_CAVE & Stock pond downstream Sanborn's Camp & $38^{\circ} 54^{\prime} 53^{\prime \prime}$ & $105^{\circ} 18^{\prime} 07^{\prime \prime}$ & ST & 11 & 1995-97 \\
\hline S3 & FLFO_NPS_BRK & Barksdale Picnic Area & $38^{\circ} 54^{\prime} 42^{\prime \prime}$ & $105^{\circ} 15^{\prime} 21^{\prime \prime}$ & ST & 11 & 1995-97 \\
\hline S4 & FLFO_NPS_HUTCH & Grape Creek upstream of FLFO & $38^{\circ} 53^{\prime} 13^{\prime \prime}$ & $105^{\circ} 15^{\prime} 59^{\prime \prime}$ & ST & 13 & $1992,95-97$ \\
\hline S5 & FLFO_EPA_CO005M & Grape Creek in FLFO & $38^{\circ} 54^{\prime} 50^{\prime \prime}$ & $105^{\circ} 16^{\prime} 42^{\prime \prime}$ & ST & 5 & 1992-95 \\
\hline S6 & FLFO_NURE_26 & $\mathrm{C} 20346$ & $38^{\circ} 55^{\prime} 27^{\prime \prime}$ & $105^{\circ} 17^{\prime} 06^{\prime \prime}$ & ST & 1 & 1976 \\
\hline S7 & FLFO_NURE_25 & C20332 & $38^{\circ} 55^{\prime} 21^{\prime \prime}$ & $105^{\circ} 16^{\prime} 55^{\prime \prime}$ & ST & 1 & 1976 \\
\hline S8 & FLFO_NURE_47 & $\mathrm{C} 20348$ & $38^{\circ} 53^{\prime} 01^{\prime \prime}$ & $105^{\circ} 15^{\prime} 27^{\prime \prime}$ & ST & 1 & 1976 \\
\hline S9 & FLFO_NURE_27 & $\mathrm{C} 20031$ & $38^{\circ} 55^{\prime} 59^{\prime \prime}$ & $105^{\circ} 13^{\prime} 29^{\prime \prime}$ & ST & 1 & 1976 \\
\hline S10 & FLFO_NURE_63 & C20033 & $38^{\circ} 54^{\prime} 47^{\prime \prime}$ & $105^{\circ} 12^{\prime} 42^{\prime \prime}$ & ST & 1 & 1976 \\
\hline S11 & FLFO_NURE_08 & C20333 & $38^{\circ} 54^{\prime} 33^{\prime \prime}$ & $105^{\circ} 16^{\prime} 46^{\prime \prime}$ & SP & 1 & 1976 \\
\hline S12 & FLFO_NURE_07 & C20347 & $38^{\circ} 54^{\prime} 38^{\prime \prime}$ & $105^{\circ} 15^{\prime} 21^{\prime \prime}$ & SP & 1 & 1976 \\
\hline S13 & FLFO_NURE_06 & C20032 & $38^{\circ} 54^{\prime} 49^{\prime \prime}$ & $105^{\circ} 14^{\prime} 57^{\prime \prime}$ & SP & 1 & 1976 \\
\hline G1 & 385445105170201 & SC0130712ABBAA (visitor center well) & $38^{\circ} 54^{\prime} 44^{\prime \prime}$ & $105^{\circ} 17^{\prime} 03^{\prime \prime}$ & GW & 1 & 1992 \\
\hline G2 & 385449105164501 & SC01307113CDDD & $38^{\circ} 54^{\prime} 48^{\prime \prime}$ & $105^{\circ} 16^{\prime} 46^{\prime \prime}$ & GW & 1 & 1991 \\
\hline
\end{tabular}


Table 3. Period of record and types of analyses conducted for surface-water samples collected near Florissant Fossil Beds National Monument, Colorado.

[value in cell is the number of samples analyzed in each category for the year; --, no data]

\begin{tabular}{|c|c|c|c|c|c|c|c|c|c|c|c|c|c|c|c|c|c|c|c|c|c|c|c|c|c|c|c|c|c|}
\hline & 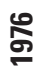 & $\tilde{\sigma}$ & $\frac{\infty}{\sigma}$ & $\frac{\mathscr{g}}{\sigma}$ & ஜீ & ळ & ฒ & ஜூ心 & 怘 & $\begin{array}{l}\text { 요 } \\
\text { Ф }\end{array}$ & $\begin{array}{l}\mathscr{0} \\
\text { g్ }\end{array}$ & ळூ & $\begin{array}{l}\infty \\
\stackrel{\infty}{\infty} \\
\stackrel{\sigma}{[}\end{array}$ & $\begin{array}{l}\text { ஜூ } \\
\text { ळ }\end{array}$ & 요 & б్ & જू & ๓๐ & 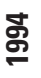 & 노 & ஜூ & 효 & $\begin{array}{l}\infty \\
\text { g్ }\end{array}$ & 요 & 용 & ్ㅇㅇ & ్ㅗㅇ & ్ㅇ & 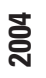 \\
\hline Field properties & 8 & -- & -- & -- & -- & -- & -- & -- & -- & -- & -- & -- & -- & -- & -- & -- & 2 & -- & 2 & 18 & 12 & 16 & -- & -- & -- & -- & _ & -- & -- \\
\hline Major constituents & 8 & -- & -- & -- & -- & -- & -- & -- & -- & -- & -- & -- & -- & -- & -- & -- & 2 & -- & 2 & 9 & 2 & 2 & -- & -- & -- & -- & -- & -- & -- \\
\hline Nutrients & -- & -- & -- & -- & -- & -- & -- & -- & -- & -- & -- & -- & -- & -- & -- & -- & 2 & -- & 2 & 7 & 2 & 2 & -- & -- & -- & -- & -- & -- & -- \\
\hline Trace elements & 8 & -- & -- & -- & -- & -- & -- & -- & -- & -- & -- & -- & -- & -- & -- & -- & 2 & -- & 2 & 2 & -- & -- & -- & -- & -- & -- & -- & -- & -- \\
\hline
\end{tabular}

phosphorus. Nitrogen compounds in all samples were low, and only a few had concentrations above laboratory reporting levels (table 4). For example, only 2 of 12 samples had detectable ammonia, and 4 of 11 samples had detectable nitrate. These low nitrogen concentrations reflect minimal human-related activities in the Grape Creek drainage basin. In contrast to nitrogen, all analyzed samples had detectable levels of phosphorus species with dissolved orthophosphate ranging from 0.14 to $0.22 \mathrm{mg} / \mathrm{L}$ and total phosphorus ranging from 0.06 to $0.28 \mathrm{mg} / \mathrm{L}$. Concentrations of the two phosphorus species were similar, indicating most of the phosphorus is present in the dissolved form. Although anthropogenic sources of phosphorus in the basin are minimal, most of the samples had phosphorus concentrations above levels typically present in undeveloped streams (Clark and others, 2000). Phosphorus to FLFO streams likely comes from natural sources, such as weathering of phosphorous-bearing minerals in lake-bed sediments and/or volcanic deposits of the Florissant Formation.

Trace elements measured in surface-water samples from FLFO are shown in table 4. Concentrations were less than laboratory reporting levels in 60 percent of the samples and only iron, manganese, and zinc had a substantial number of detected concentrations greater than 10 micrograms per liter $(\mu \mathrm{g} / \mathrm{L})$. The largest trace element concentrations were measured at eight sites (S6-S13, fig. 2) sampled by the NURE program. The reason for larger concentrations in the NURE samples is unclear; however, because of the age of the data (more than 25 years old) and the lack of published qualityassurance information for water samples (Sharp and Aamodt, 1978), the NURE data should be interpreted with care.

Other constituents measured in FLFO surface-water samples included dissolved organic carbon ( 4.3 to $11 \mathrm{mg} / \mathrm{L}$ ), fecal coliform [7 and 21 colonies per 100 milliliters $(\mathrm{col} / 100 \mathrm{~mL})$ ] and total coliform (0 to $3,000 \mathrm{col} / 100 \mathrm{~mL}$ ). Concentrations of these constituents were in the range expected for small streams draining undeveloped basins.

Because there are few water-quality data available for FLFO, little can be described about spatial and seasonal variations in water quality within the park. The most complete data set available includes core field properties (water temperature, dissolved oxygen, $\mathrm{pH}$, and specific conductance), which were measured at four sites from 1995 to 1997 (fig. 4). Dissolved oxygen concentrations ranged from 5.0 to $12.3 \mathrm{mg} / \mathrm{L}$ and did not show a consistent seasonal pattern at any of the four sites.
The largest concentrations typically were measured at S2, which is a small stock pond along a tributary. Elevated dissolved oxygen at this site may be because of photosynthesis by aquatic vegetation in the pond, causing dissolved oxygen concentrations and $\mathrm{pH}$ in surface water to increase during the day. $\mathrm{pH}$ values ranged from 6.56 to 9.40 and were lowest at site S3, downstream from the spring, and highest at site S2, a manmade stock pond, which also had the largest dissolved oxygen concentration. All four sites showed similar seasonal patterns with lower concentrations in the spring and higher in the fall. Specific conductance at the four sites ranged from 184 to $510 \mu \mathrm{S} / \mathrm{cm}$ and was highest at site $\mathrm{S} 1$, which is the furthest downstream site. Specific conductance at sites S1 and S4 indicated similar seasonal patterns of lower concentrations in spring because of dilution of stream water by snowmelt. By contrast, specific conductance showed little seasonal variation at site $\mathrm{S} 3$, probably because it is fed by discharge from a nearby ground-water spring. None of the surface-water sites had a sufficient period of record to test for water-quality changes with time using ESTREND.

Comparison to Water-Quality Standards. Water-quality use classifications and standards for all stream segments in Colorado are established by the Colorado Water Quality Control Commission (Colorado Department of Public Health and Environment, 2006). Grape Creek and its tributaries are classified under segment 2a of the Upper South Platte River Basin. None of the reaches within this segment were listed in 2006 as impaired for water quality (http://www.cdphe.state.co.us/ op/wqcc/SpecialTopics/303(d)/303dtmdlpro.html, accessed May 2006). The designated uses for this segment are coldwater aquatic life class 1 , recreation 1a, agriculture, and water supply. Streams designated for cold-water aquatic life class 1 (the most sensitive use) should have temperatures less than 20 ${ }^{\circ} \mathrm{C}$, dissolved oxygen greater than $6.0 \mathrm{mg} / \mathrm{L}(7.0 \mathrm{mg} / \mathrm{L}$ during spawning), and $\mathrm{pH}$ values in the 6.5 to 9.0 range. In FLFO, 48 of 50 stream temperatures were less than the $20^{\circ} \mathrm{C}$ standard, 34 of 38 dissolved oxygen measurements were greater than the $6.0 \mathrm{mg} / \mathrm{L}$ standard, and 40 of $50 \mathrm{pH}$ values were within the 6.5 to $9.0 \mathrm{pH}$ range. Most of the values that exceeded the aquatic-life standards were from site $\mathrm{S} 2$, which is a manmade stock pond on Grape Creek. Concentrations of nutrients and major constituents (chloride and sulfate) in all samples were below the water-supply and cold-water aquatic life standards, except for one stream sample that exceeded the ammonia 
Table 4. Summary of selected water-quality data for surface-water sites in Florissant Fossil Beds National Monument, Colorado, 1976 to 1997.

[no., number; chronic aquatic-life (and water-supply) standards from Colorado Department of Public Health and Environment (2006); <, less than; >, greater than; --, not reported; ${ }^{\circ} \mathrm{C}$, degrees Celsius; $\mathrm{mg} / \mathrm{L}$, milligrams per liter; $\mu \mathrm{S} / \mathrm{cm}$, microsiemens per centimeter at $25^{\circ} \mathrm{C} ; \mathrm{CaCO}_{3}$, calcium carbonate; $\mathrm{N}$, nitrogen; P, phosphorous; col/100 mL, colonies per 100 milliliters; $\mu \mathrm{g} / \mathrm{L}$, micrograms per liter; some nutrients and trace elements have multiple reporting limits]

\begin{tabular}{|c|c|c|c|c|c|c|}
\hline Constituent or property & $\begin{array}{l}\text { No. } \\
\text { sites }\end{array}$ & $\begin{array}{l}\text { No. analyses } \\
\text { (no. censored) }\end{array}$ & $\begin{array}{l}\text { Minimum } \\
\text { value }\end{array}$ & $\begin{array}{c}\text { Median } \\
\text { value }\end{array}$ & $\begin{array}{l}\text { Maximum } \\
\text { value }\end{array}$ & $\begin{array}{c}\text { Aquatic-life } \\
\text { (water-supply) } \\
\text { standard }\end{array}$ \\
\hline \multicolumn{7}{|c|}{ Field properties } \\
\hline Temperature, water $\left({ }^{\circ} \mathrm{C}\right)$ & 13 & 50 & 5.6 & 12.8 & 22.2 & $<20$ \\
\hline Oxygen, dissolved (mg/L) & 5 & 38 & 5.0 & 8.0 & 12.3 & $>6.0(>3.0)$ \\
\hline $\mathrm{pH}$ (standard units) & 13 & 50 & 6.56 & 7.72 & 9.40 & $6.5-9.0(5.0-9.0)$ \\
\hline Specific conductance $(\mu \mathrm{S} / \mathrm{cm})$ & 13 & 50 & 130 & 273 & 525 & -- \\
\hline \multicolumn{7}{|c|}{ Major constituents } \\
\hline Alkalinity $\left(\mathrm{mg} / \mathrm{L}\right.$ as $\left.\mathrm{CaCO}_{3}\right)$ & 5 & 15 & 66 & 140 & 180 & -- \\
\hline Calcium, dissolved (mg/L) & 10 & 14 & 20 & 44 & 84 & -- \\
\hline Chloride, dissolved (mg/L) & 5 & $13(3)$ & a 4.0 & 5.8 & 20 & (b250) \\
\hline Fluoride, dissolved (mg/L) & 1 & 4 & 2.2 & 2.3 & 2.5 & $(2.0)$ \\
\hline Magnesium, dissolved (mg/L) & 10 & 14 & 3.7 & 7.3 & 16 & -- \\
\hline Potassium, dissolved (mg/L) & 2 & 6 & 2.4 & 6.8 & 7.8 & -- \\
\hline Silica, dissolved (mg/L) & 0 & 0 & -- & -- & -- & -- \\
\hline Sodium, dissolved (mg/L) & 2 & 6 & 7.6 & 12 & 17 & -- \\
\hline Sulfate, dissolved (mg/L) & 2 & 6 & 6.7 & 35 & 73 & (b250) \\
\hline \multicolumn{7}{|c|}{ Nutrients, carbon, and microorganisms } \\
\hline Ammonia, dissolved (mg/L as $\mathrm{N}$ ) & 3 & $12(10)$ & $<0.04$ & $<0.10$ & 0.43 & 0.02 \\
\hline Nitrate, dissolved (mg/L as $\mathrm{N})$ & 3 & $11(7)$ & $<.05$ & $<1.0$ & $<1.0$ & (10) \\
\hline Nitrite, dissolved (mg/L as $\mathrm{N}$ ) & 1 & $1(1)$ & $<.02$ & -- & -- & $.05(1.0)$ \\
\hline Orthophosphate, dissolved (mg/L as $\mathrm{P}$ ) & 1 & 4 & .14 & .19 & .22 & -- \\
\hline Phosphorus, total (mg/L as $\mathrm{P}$ ) & 5 & 11 & .06 & .17 & .28 & -- \\
\hline Organic carbon, dissolved (mg/L) & 1 & 4 & 4.3 & 5.5 & 10 & -- \\
\hline Fecal coliform (col/100 mL) & 2 & 2 & 7 & -- & 21 & -- \\
\hline Total coliform (col/100 mL) & 2 & 4 & 0 & 153 & 3,000 & -- \\
\hline \multicolumn{7}{|c|}{ Trace elements } \\
\hline Aluminum, dissolved $(\mu \mathrm{g} / \mathrm{L})$ & 1 & $4(4)$ & $<30$ & $<30$ & $<30$ & c87 \\
\hline Arsenic, dissolved $(\mu \mathrm{g} / \mathrm{L})$ & 1 & $4(2)$ & $<4$ & 4.3 & 6 & ${ }^{\mathrm{c}} 50(10)$ \\
\hline Cadmium, dissolved $(\mu \mathrm{g} / \mathrm{L})$ & 1 & $4(4)$ & $<0.5$ & $<.5$ & $<.5$ & d.6(5) \\
\hline Chromium, dissolved ( $\mu \mathrm{g} / \mathrm{L})$ & 9 & $12(9)$ & $<1.5$ & $<25$ & 37 & $11(50)$ \\
\hline Cobalt, dissolved ( $\mu \mathrm{g} / \mathrm{L})$ & 8 & $8(8)$ & $<55$ & $<55$ & $<55$ & -- \\
\hline Copper, dissolved ( $\mu \mathrm{g} / \mathrm{L})$ & 9 & $12(9)$ & $<.6$ & $<4$ & $<4$ & ${ }^{\mathrm{d}} 13(1,300)$ \\
\hline Iron, dissolved $(\mu \mathrm{g} / \mathrm{L})$ & 9 & 12 & 14 & 108 & 1,225 & ${ }^{\mathrm{c}} 1,000\left({ }^{\mathrm{b}} 300\right)$ \\
\hline Lead, dissolved $(\mu \mathrm{g} / \mathrm{L})$ & 9 & $12(12)$ & $<4$ & $<200$ & $<200$ & $\mathrm{~d} 4.2(50)$ \\
\hline Manganese, dissolved ( $\mu \mathrm{g} / \mathrm{L})$ & 9 & $12(3)$ & $<3$ & 15 & 353 & ${ }^{\mathrm{d}} 1,930\left({ }^{\mathrm{b}} 50\right)$ \\
\hline Nickel, dissolved ( $\mu \mathrm{g} / \mathrm{L})$ & 9 & $12(9)$ & $<12$ & $<25$ & 110 & d77 (100) \\
\hline Selenium, dissolved $(\mu \mathrm{g} / \mathrm{L})$ & 1 & $4(4)$ & $<5$ & $<5$ & $<5$ & $4.6(50)$ \\
\hline Silver, dissolved $(\mu \mathrm{g} / \mathrm{L})$ & 1 & $4(4)$ & $<.3$ & $<.3$ & $<.3$ & d.7 (b 100) \\
\hline Uranium, dissolved $(\mu \mathrm{g} / \mathrm{L})$ & 8 & 8 & .78 & 2.0 & 16.2 & ${ }^{\mathrm{d}} 2,520(30)$ \\
\hline Zinc, dissolved $(\mu \mathrm{g} / \mathrm{L})$ & 9 & $12(4)$ & $<4$ & 114 & 371 & ${ }^{\mathrm{d}} 186\left({ }^{\mathrm{b}} 5,000\right)$ \\
\hline
\end{tabular}

${ }^{a}$ Minimum reported value less than minimum censored value.

${ }^{\mathrm{b}}$ Secondary standard based on aesthetic properties such as taste, odor, and staining.

'Total recoverable concentration.

dTable value standard calculated for a hardness of $160 \mathrm{mg} / \mathrm{L}$. 

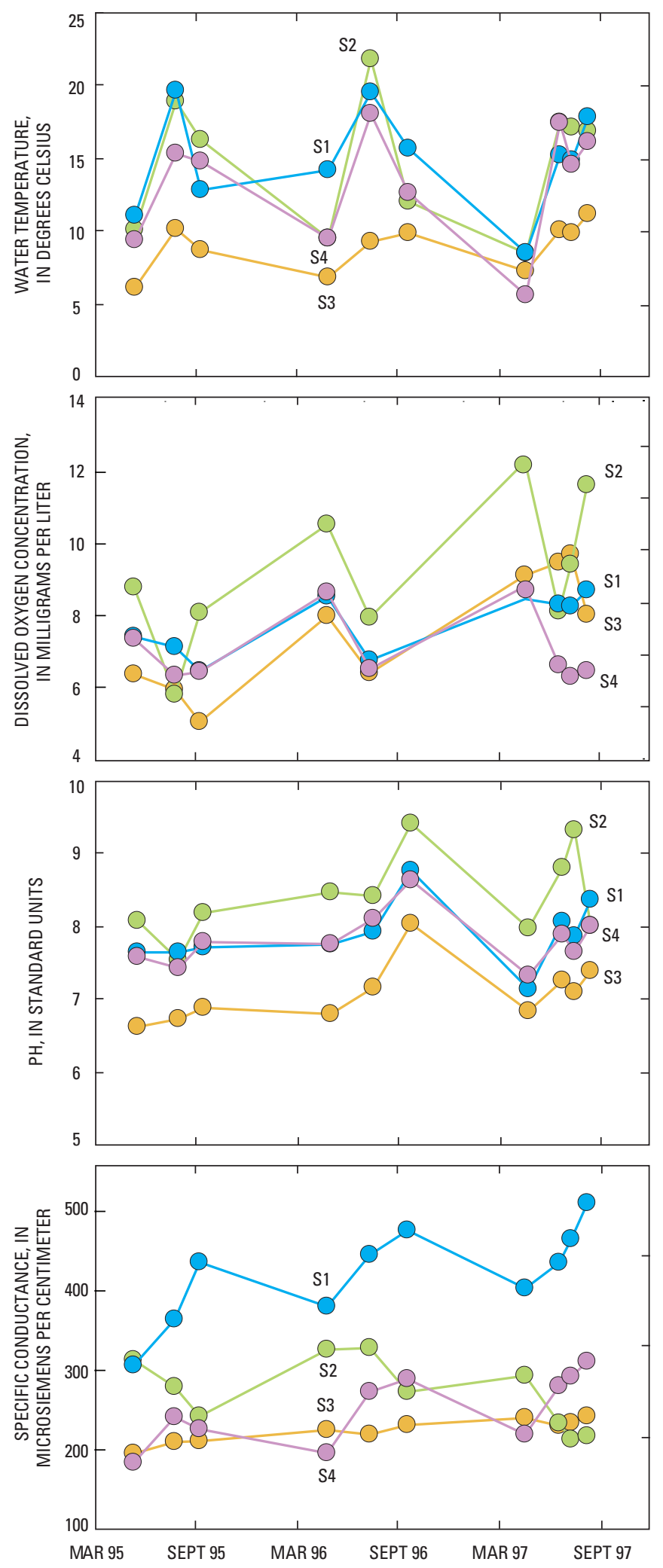

Figure 4. Seasonal variation in water quality at selected sites in Florissant Fossil Beds National Monument, Colorado. standard. There were only two fecal coliform measurements (7 and $21 \mathrm{col} / 100 \mathrm{~mL}$ ), but neither exceeded the $200 \mathrm{col} / 100 \mathrm{~mL}$ standard for recreation 1a. Two samples collected in the Grape Creek drainage at sites S8 and S13 exceeded the chronic aquatic-life standards for chromium, iron, nickel and zinc, which were calculated from table value standards for a median hardness of $160 \mathrm{mg} / \mathrm{L}$. However, these samples were collected more than 25 years ago as part of the NURE program and quality-assurance information was not published so the data should be interpreted with care.

\section{Ground-Water Quality}

Water-quality data are available for two ground-water wells (G1 and G2, fig. 2) in FLFO. Site G1 is a well used for drinking water at the park visitor center and was sampled by the USGS on July 15, 1992; site G2 was sampled by the USGS on June 20, 1991. Major dissolved constituents in the two ground-water samples were present in the same proportions as in surface-water samples, although concentrations were as much as two times higher (table 5). As observed in surface-water samples, fluoride concentrations in groundwater samples were greater than $1.0 \mathrm{mg} / \mathrm{L}$, which may reflect the high-fluoride content of the bedrock. The fluoride concentration at site G1 was $3.1 \mathrm{mg} / \mathrm{L}$, which was above the Colorado water-supply standard of $2.0 \mathrm{mg} / \mathrm{L}$, but below the USEPA maximum contaminant level of $4.0 \mathrm{mg} / \mathrm{L}$ ( $h t t p: / / w w w$. epa.gov/safewater/contaminants/index.html, accessed May 2006). Nutrient concentrations in ground-water samples were well below the water-supply standards for nitrate and nitrite. Most of the 13 trace elements had concentrations at or close to laboratory reporting levels with the exception of iron, lithium, manganese, and zinc. Site G2 had elevated iron $(2,122 \mu \mathrm{g} / \mathrm{L})$ and manganese $(493 \mu \mathrm{g} / \mathrm{L})$ concentrations that exceeded the secondary standards for drinking water. Elevated iron and manganese in ground water often is because of dissolution of oxide minerals in aquifer materials when dissolved oxygen concentrations become depleted (Hem, 1985). The high lithium concentrations were unusual and, similar to fluoride, likely are related to contributions from natural weathering sources.

\section{Summary and Suggestions for Vital Signs Monitoring}

Review of historical water-quality records indicates that few data are available for FLFO and most of what is available is more than a decade old. From 1976 to 1997, 60 waterquality samples were collected at 13 surface-water sites. The majority of results for these 60 samples were field properties (39 percent), and major-constituent (23 percent), nutrient ( 8 percent), and trace-element (29 percent) analyses. Only five of the sites were sampled more than once during the period of record, and mostly for field properties only. None of the streams in FLFO have been gaged for streamflow and manual 
Table 5. Summary of selected water-quality data for ground-water sites in Florissant Fossil Beds National Monument, Colorado.

[water-supply standards from Colorado Department of Public Health and Environment (2006); <, less than; --, not reported; $\mu \mathrm{S} / \mathrm{cm}$, microsiemens per centimeter at $25^{\circ} \mathrm{C} ; \mathrm{mg} / \mathrm{L}$, milligrams per liter; $\mathrm{CaCO}_{3}$, calcium carbonate; $\mathrm{N}$, nitrogen; P, phosphorous; $\mu \mathrm{g} / \mathrm{L}$, micrograms per liter]

\begin{tabular}{|c|c|c|c|}
\hline Constituent or property & $\begin{array}{l}\text { G1 (fig. 2) } \\
7 / 15 / 1992\end{array}$ & $\begin{array}{l}\text { G2 (fig. 2) } \\
6 / 20 / 1991\end{array}$ & Water-supply standard \\
\hline \multicolumn{4}{|c|}{ Field properties } \\
\hline $\mathrm{pH}$ (standard units) & 7.30 & 7.25 & $5.0-9.0$ \\
\hline Specific conductance $(\mu \mathrm{S} / \mathrm{cm})$ & 496 & 871 & -- \\
\hline \multicolumn{4}{|c|}{ Major constituents } \\
\hline Alkalinity $\left(\mathrm{mg} / \mathrm{L}\right.$ as $\left.\mathrm{CaCO}_{3}\right)$ & 218 & 349 & -- \\
\hline Calcium, dissolved (mg/L) & 62 & 98 & -- \\
\hline Chloride, dissolved (mg/L) & 8.5 & 39 & a250 \\
\hline Fluoride, dissolved (mg/L) & 3.1 & 1.1 & 2.0 \\
\hline Magnesium, dissolved (mg/L) & 12 & 25 & -- \\
\hline Potassium, dissolved (mg/L) & 4.4 & 10 & -- \\
\hline Silica, dissolved (mg/L) & 23 & 54 & -- \\
\hline Sodium, dissolved (mg/L) & 21 & 52 & -- \\
\hline Sulfate, dissolved (mg/L) & 32 & 67 & a250 \\
\hline \multicolumn{4}{|c|}{ Nutrients } \\
\hline Ammonia (mg/L as $\mathrm{N})$ & 0.03 & 0.28 & -- \\
\hline Nitrate $(\mathrm{mg} / \mathrm{L}$ as $\mathrm{N})$ & $<.05$ & .096 & 10 \\
\hline Nitrite (mg/L as N) & $<.01$ & $<.01$ & 1.0 \\
\hline Orthophosphate, dissolved ( $\mathrm{mg} / \mathrm{L}$ as $\mathrm{P}$ ) & $<.01$ & .09 & -- \\
\hline Phosphorus, total (mg/L as $\mathrm{P}$ ) & $<.01$ & .09 & -- \\
\hline \multicolumn{4}{|c|}{ Trace elements } \\
\hline Cadmium, dissolved $(\mu \mathrm{g} / \mathrm{L})$ & $<1$ & $<1$ & 5 \\
\hline Chromium, dissolved ( $\mu \mathrm{g} / \mathrm{L})$ & $<5$ & $<5$ & 50 \\
\hline Cobalt, dissolved ( $\mu \mathrm{g} / \mathrm{L})$ & $<3$ & $<3$ & -- \\
\hline Copper, dissolved $(\mu \mathrm{g} / \mathrm{L})$ & $<10$ & $<10$ & 1,000 \\
\hline Iron, dissolved $(\mu \mathrm{g} / \mathrm{L})$ & 200 & 2,122 & a300 \\
\hline Lead, dissolved $(\mu \mathrm{g} / \mathrm{L})$ & $<10$ & 13 & 50 \\
\hline Lithium, dissolved $(\mu \mathrm{g} / \mathrm{L})$ & 75 & 142 & -- \\
\hline Manganese, dissolved ( $\mu \mathrm{g} / \mathrm{L})$ & 130 & 493 & a50 \\
\hline Molybdenum, dissolved ( $\mu \mathrm{g} / \mathrm{L})$ & $<10$ & $<10$ & -- \\
\hline Nickel, dissolved ( $\mu \mathrm{g} / \mathrm{L})$ & $<10$ & $<10$ & 100 \\
\hline Silver, dissolved $(\mu \mathrm{g} / \mathrm{L})$ & $<1$ & $<1$ & a 100 \\
\hline Vanadium, dissolved $(\mu \mathrm{g} / \mathrm{L})$ & $<6$ & $<6$ & -- \\
\hline Zinc, dissolved $(\mu \mathrm{g} / \mathrm{L})$ & 410 & $<3$ & a5,000 \\
\hline
\end{tabular}

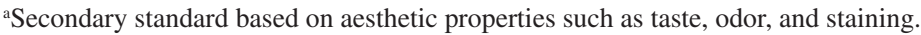


discharge measurements were made only periodically at two sites in the park. Surface water in the area is a calciumbicarbonate type and dissolved solids primarily are derived from weathering of the underlying granite and lake-bed sediments. Land-use activities likely have a minor effect on water quality, although there is concern about future increases in visitor use and residential development in areas upstream from the park. Because of a lack of long-term monitoring sites, it was not possible to identify temporal trends in water quality. Designated uses for streams in FLFO are cold-water aquatic life class 1, recreation 1a, agriculture, and water supply; the available water-quality data indicate most sites meet Colorado water-quality standards for cold-water aquatic life class 1 (the most sensitive use). Water-quality data are available for one sample at each of the two ground-water wells in the park. One of the wells exceeded the drinking-water standard for fluoride and the other well exceeded secondary standards for manganese and iron. Elevated fluoride in FLFO is attributed to natural weathering of fluoride-rich bedrock that underlies the park.

Issues of concern for water resources in the park include visitor use, land-use activities adjacent to the park, and climate change. Based on a review of the historical data, the following suggestions are provided for consideration in designing a water-quality monitoring plan for FLFO.

- Establish a long-term water-quality monitoring station or "sentinel site" on Grape Creek at the northern park boundary. Monitor for core field properties, major constituents, nutrients, bacterial indicators, and suspended sediment during different hydrologic conditions. Because of the small drainage area of Grape Creek, water samples collected at the sentinel site should be representative of water-quality conditions in the park.

- Establish a continuous streamflow-monitoring station collocated with the Grape Creek sentinel site. Streamflow monitoring is important for interpreting trends in water quality and for monitoring the effects of human stressors, such as residential development and climate change, on water resources.

- Sites upstream from the sentinel site, including groundwater springs, could be sampled periodically to address special issues or to achieve a better understanding of the factors controlling water quality in the park.

- Ground-water levels or spring discharge could be used to monitor the effects of human stressors on water resources.

\section{Glacier National Park}

\section{Environmental Setting and Geology}

Glacier National Park (GLAC) is located in the northern Rocky Mountains in northwestern Montana and is bounded to the north by Canada (fig. 5). The North and Middle Forks of the Flathead River border the park on the west and south, and the park is bisected by the Continental Divide. The park covers $4,100 \mathrm{~km}^{2}$, ranges in elevation from 949 to $3,180 \mathrm{~m}$, and is characterized by horn-shaped peaks, broad U-shaped valleys, numerous glacial lakes, and several active glaciers. Five large bioregions are present in GLAC including alpine tundra, subalpine forest, montane forest, aspen parkland, and fescue grassland. Coniferous forests dominate much of the landscape with lodgepole pine, Douglas fir, and western larch growing on drier sites and western hemlock and western red cedar inhabiting wetter sites (White and others, 1998). The climate in GLAC is one of long, cold, snowy winters and relatively short, warm summers. The west side of the park is affected by Pacific air masses that result in a milder and moister climate than the east side, which is dominated by colder Continental air masses. Winter temperatures are warmer on the west side than the east, but summer temperatures are similar (Finklin, 1986). Annual precipitation differs substantially between westside and east-side locations in the park and ranges from as little as $30 \mathrm{~cm}$ at low elevations on the east side to as much as $350 \mathrm{~cm}$ at higher elevations on the west side (Finklin, 1986). Most precipitation in winter falls as snow that accumulates in a seasonal snowpack between November and April.

GLAC is underlain by the Proterozoic Belt Supergroup, which consists of thick sequences of argillite, quartzite, and carbonate rocks (Ross, 1959). Although the rocks are thoroughly consolidated and recrystallized, they contain well-preserved sedimentary features such as ripple marks, mud cracks, and stromatolite fossils. The primary units in the Supergroup are the Altyn Limestone, Appekunny Argillite, Grinnell Argillite, Helena Dolomite, and Missoula Group. Sediments in the Belt Supergroup were folded and faulted during the Paleocene producing the Lewis overthrust, which is a large thrust fault that is exposed along the eastern edge of the park (Ross, 1959). During the Pleistocene, valley glaciers and ice fields covered the region carving the glacial topography seen today. As the glaciers retreated, the stream and river valleys were filled with poorly sorted glacial till, outwash, and alluvium derived from the sedimentary bedrock. In 2005, GLAC had 27 active glaciers that are direct remnants from a period of glacier formation ending about 11,000 years ago. The glaciers in the park have been shrinking because of climate change, and at current rates it is estimated that all glaciers in the park will be gone by the year 2030 (Fagre and others, 2005).

Land-use activities in the park are related to tourism and park administration including the road system, campgrounds, picnic areas, boat launches, liveries, visitor centers, lodges, and park administrative buildings and residences. The park has approximately 1.8 million visitors each year, mostly between June and September (Britten and others, 2006). More than 90 percent of park lands are designated wilderness, and human activities in these areas are limited. Water supply in the park is obtained from a system of 7 surface-water sources and 20 ground-water sources. Water supplies are tested twice monthly during the high-use season for indicator bacteria and nitrogen species according to water-supply regulations set by 


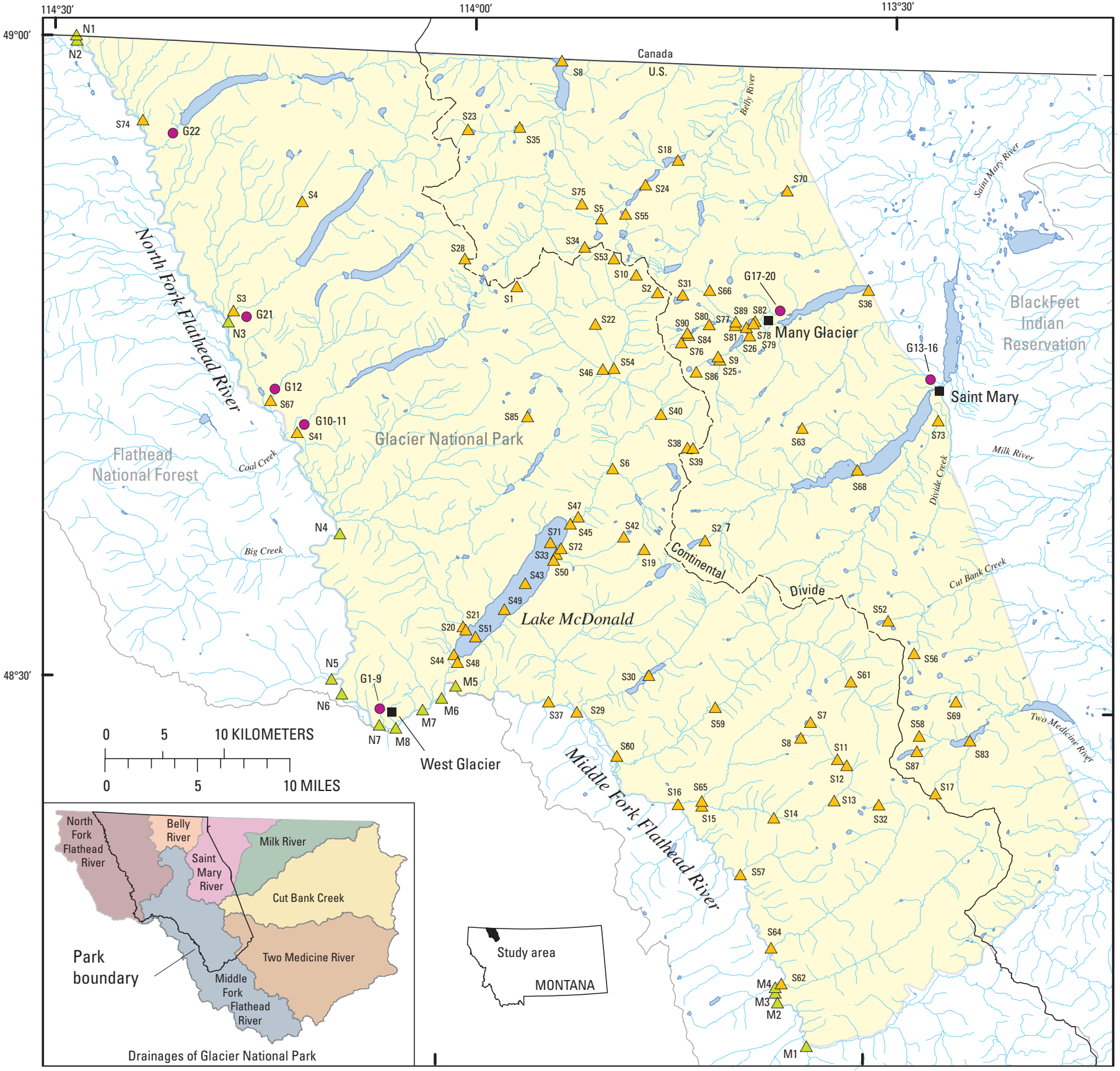

Base from National Hydrography Dataset, 1:100,000 Universal Transverse Mercator projection Zone 12

\section{EXPLANATION}

S64 Stream and lake sampling site and number (table 6)

M1 $\triangle$ Mainstem sampling site and number (table 8)

61-9 Observation well

and number (table 12)

- Developed area

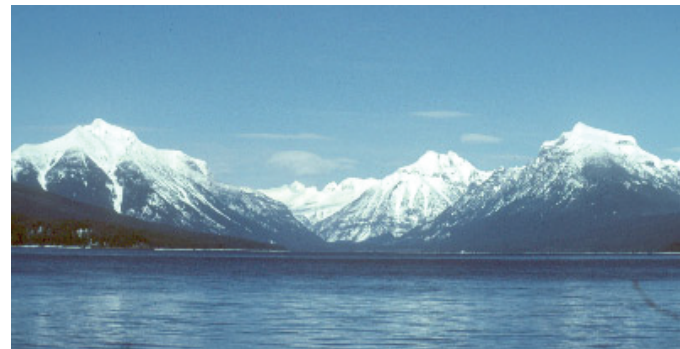

Photograph of Lake McDonald by Karen Holzer, U.S. Geological Survey

Figure 5. Location of water-quality sampling sites in Glacier National Park, Montana. 
the MTDEQ. Results of water-supply testing are available at http://www.deq.state.mt.us/wqinfo/pws/reports.asp (accessed November 2006). Treatment facilities at West Glacier, Saint Mary, and Many Glacier (fig. 5) and several small septic systems handle wastewater for park facilities. Prescribed burns are conducted periodically in the park to improve habitat and to reduce fuels. An average of 14 natural wildfires have burned $20 \mathrm{~km}^{2}$ in the park each year since 1988 (http://www. nps.gov/archive/glac/resources/fires.htm, accessed November 2006). GLAC is bounded by largely undeveloped National Forest land to the west and south and by the Blackfeet Indian Reservation to the east. Land-use activities in areas adjacent to GLAC include timber harvesting, low-density residential development, road networks, ranching, and mineral exploration (Hauer and others, 2007).

\section{Water Resources}

GLAC is straddled by the Continental Divide and encompasses the headwaters of three continental river systems; the Columbia (North Fork and Middle Fork Flathead River), the Missouri (Milk River, Cut Bank Creek, Two Medicine River), and the Saskatchewan (Belly River and Saint Mary River). Drainages on the west side of the park flow into the North and Middle Forks of the Flathead River, which form the southern and western park boundaries (fig. 5). Drainages on the east side of the park form the headwaters of the Belly, Saint Mary, and Two Medicine Rivers. The park contains more than 2,500 $\mathrm{km}$ of perennial streams (Britten and others, 2006) that flow through glacially carved drainages, some of which still contain active glaciers in their headwaters. The park contains more than 650 lakes; the largest is Lake McDonald on the west side of the park, which is $16 \mathrm{~km}$ long and has a maximum depth of $144 \mathrm{~m}$ (fig. 5).

The USGS has operated as many as 25 streamflowgaging stations inside or adjacent to the boundaries of the park (http://mt.water.usgs.gov/pub/MTStations.pdf, accessed November 2006). Of these, six stations currently (2007) are active including Swiftcurrent Creek above Swiftcurrent Lake (USGS station 05014300; site S78 in figure 5), Swiftcurrent Creek at Many Glacier (USGS station 05014500; S81), Lake Sherburne (USGS station 05015500; S36), Middle Fork Flathead River (USGS station 12358500; M6), Flathead River at Flathead (USGS station 12355000; N2), and North Fork Flathead River (USGS station 12355500; N6). Swiftcurrent Creek above Swiftcurrent Lake (S78) is part of the USGS Hydrologic Benchmark Network, a National program for monitoring streamflow and water quality in areas that are minimally affected by human activities (Murdoch and others, 2005). The longest continuously operating station in the park is Swiftcurrent Creek at Many Glacier (S81), which began operation in 1912. The range of daily streamflows at Swiftcurrent Creek at Many Glacier from 1986 to 2005 is shown in figure 6. Peak streamflow occurs during spring snowmelt in late May or June during which time streamflow increases by two to three orders of magnitude over winter streamflows. More than 60 percent of the annual streamflow occurs from mid May through mid July. Streamflow decreases steadily through the remainder of the year reaching a minimum in December, January, and February when streamflow is sustained by slow release of ground water and storage in lakes.

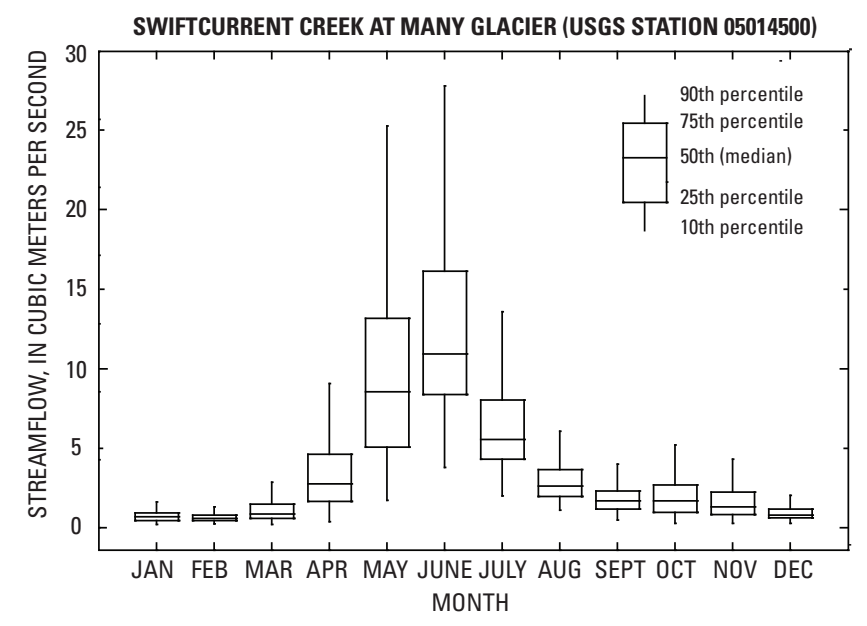

Figure 6. Daily streamflow range at Swiftcurrent Creek at Many Glacier (S81) in Glacier National Park, Montana, 1986-2005.

In mountainous areas such as GLAC, there generally are two different ground-water systems; bedrock aquifers in the mountain block, and shallow localized aquifers in alluvium and glacial till along stream and river channels. In GLAC, the hydrologic characteristics of mountain block aquifers are virtually unknown, and sparse information is available for shallow alluvial aquifers. The NPS had observation wells (fig. 5) installed in the vicinity of three wastewater treatment facilities in the park and several campgrounds and ranger stations. All the wells were shallow ( 3.1 to $24.4 \mathrm{~m}$ ) and were completed in unconsolidated alluvial or glacial deposits composed of clay, silt, sand, and gravel (Boettcher, 1973). Because of the poorly sorted nature of these deposits, the water-bearing characteristics vary widely from place to place. Aquifer tests on several of the campground wells indicated yields between 0.25 to 3.2 liters per second (L/s) (Boettcher, 1973). Weekly to monthly water-level measurements were made at observation wells at West Glacier, Saint Mary, and Many Glacier wastewater treatment facilities (Moreland and Wood, 1982). Water levels for the Many Glacier wells (G17 to G20) from May 1980 to August 1982 are shown in figure 7. Water levels varied substantially during the year and peaked in June indicating alluvial aquifers are recharged primarily by snowmelt and respond quickly to changes in streamflow.

The park has a National Atmospheric Deposition Program (NADP) station located at West Glacier that has been operated by the NPS since 1980 . The NADP is a national network of precipitation monitoring sites that measures weekly precipitation chemistry and precipitation amount; data for the network are available at $h t t p: / / n a d p . s w s . u i u c . e d u /$ (accessed November 2006). The site at GLAC is used to monitor the 


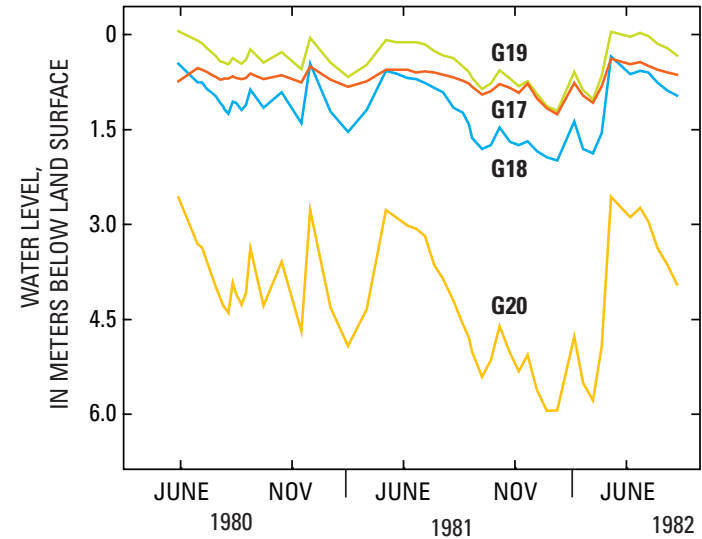

Figure 7. Periodic water-level measurements at selected shallow ground-water wells in Glacier National Park, Montana.

effect of air pollution on ecological resources including vegetation, water quality, and soils (http://www2.nature.nps.gov/ air/index.cfm, accessed November 2006). The park also has a Clean Air Status and Trends (CASNET) site at 976 m, not far from the West Glacier NADP station. CASTNET is a national network of air monitoring sites that provide estimates of dry deposition of sulfur and nitrogen compounds and concentrations of ground-level ozone (http://www.epa.gov/castnet/, accessed November 2006). The CASNET site in GLAC is operated by the NPS and was added to the network in 1990.

Water-quality concerns for GLAC may include effects of visitor use, park facilities, and road networks on nutrients, sediment, and bacteria. Sedimentation and erosion associated with flooding following prescribed burns and large-scale wildfires also may be a concern. Land-use activities outside the park boundary such as timber harvesting, road networks, and mineral exploration may affect water quality of the Middle and North Forks of the Flathead River (Hauer and others, 2007). Long-term increases in atmospheric deposition of contaminants (particularly nitrogen) may pose a threat to high-elevation lakes and streams (Williams and others, 1996). Climate change is causing the rapid retreat of park glaciers, and may have long-term effects on surface- and ground-water resources in the park (Fagre and others, 2005).

\section{Surface-Water Quality}

For this report, water-quality data were compiled for 90 surface-water sites originating within the boundaries of GLAC including 54 stream sites and 36 lake sites (table 6, fig. 5). The types of analyses and numbers of samples collected for the period of record for these 90 sites are summarized in table 7. The ROMN database contains water-quality data for 1,144 samples at these sites collected over the period 1963 to 2004, the majority of which were field properties (19 percent), and major-constituent (30 percent), nutrient (26 percent), traceelement (8 percent), and organic carbon ( 7 percent) analyses.
Most of the surface-water sites were sampled 1 to 3 times each during the period of record, although there were 14 sites that were sampled 20 or more times (table 6). The majority of these sites were sampled by the USEPA, Flathead Lake Biological Station (FLBS), or the USGS. The USEPA collected 102 samples from 10 sites (S20, S21, S33, S43, S44, S45, S50, S51, S71, S72) during water year 1975 as part of a lake eutrophication study. Five lake sites (S1, S17, S19, S24, S30) were sampled by USEPA in 1985 as part of the Western Lake Survey (Landers and others, 1987). The FLBS collected 149 samples from 14 lake sites (S7, S17, S27, S28, S42, S49, S52, S68, S69, S75, S82, S83, S85, S88) from 1984-1990 as part of a cooperative monitoring program with the NPS. The objective of the program was to document annual variability in water quality, physical characteristics, and phytoplankton and zooplankton communities for selected lakes in the park (Ellis and others, 1992; Ellis and others, 2003). In a separate study, the FLBS collected 357 samples during 1992 to 1994 from 14 stream sites (S2, S6, S10, S21, S22, S34, S38, S39, S40, S46, S47, S48, S53, S54) in the McDonald Creek drainage and 2 sites on Lake McDonald (S50, S51). The samples were collected from 1992 to 1998 and were analyzed primarily for nutrient species in a cooperative study with the NPS (Hauer and others, 2003). The USGS collected water-quality data for 478 samples from 55 stream and lake sites from 1963 to 2004. More than 70 percent of these samples were collected at 14 sites (S9, S25, S26, S31, S66, S76-S81, S86, S89, S90) in the Swiftcurrent Creek drainage on the east side of the park as part of the USGS Hydrologic Benchmark Network (Clark and others, 1999) including 129 samples from Swiftcurrent Creek at Many Glacier (S81) and 187 samples from Swiftcurrent Creek above Swiftcurrent Lake (S78). The USGS began a project in 2004 to assess the effects of the 2003 wildfires on water quality in the park. As part of this project, major constituent and nutrient data have been collected as frequently as weekly at Coal Creek (S15) and Pinchot Creek (S65) sites, and intermittently at 14 additional stream sites (S8, S11-S14, S16, S29, S37, S57, S59-S62, S64). Five lakes (S1, S17, S18, S24, S30) were sampled by the USEPA in 1985 during the Western Lake Survey (Landers and others, 1987) and six lake sites (S17, S18, S19, S24, S30, S55) were sampled by the USGS during a lake survey in 1999 (Clow and others, 2002). Ten lake sites (S4, S5, S35, S42, S55, S58, S63, S70, S75, S84) were sampled during the summers of 2002 or 2003 by the USGS to document the distribution and occurrence of organochlorine compounds and pesticides in lake sediments (Mast and others, 2006). Miscellaneous field measurements were made by the USGS at sites S3, S41, S67, and S74 in the late 1980s.

In addition to the 90 stream and lake sites originating inside the park boundary, water-quality samples were collected at 7 sites on the main stem of the North Fork of the Flathead River and 8 sites on the Middle Fork of the Flathead River, which flow along the park boundary (fig. 5 and table 8). On the North Fork, the USGS collected water-quality samples at sites N2 and N6. Site N1, which is in Canada just upstream from site N2, was sampled monthly by Environment 
Table 6. Surface-water sampling sites in Glacier National Park, excluding sites on the North and Middle Forks of the Flathead River, Montana.

[no., number; LK, lake; ST, stream; GNP, Glacier National Park; Cr, Creek; ab, above; nr, near; identification numbers and station names from USEPA STORET and USGS NWIS]

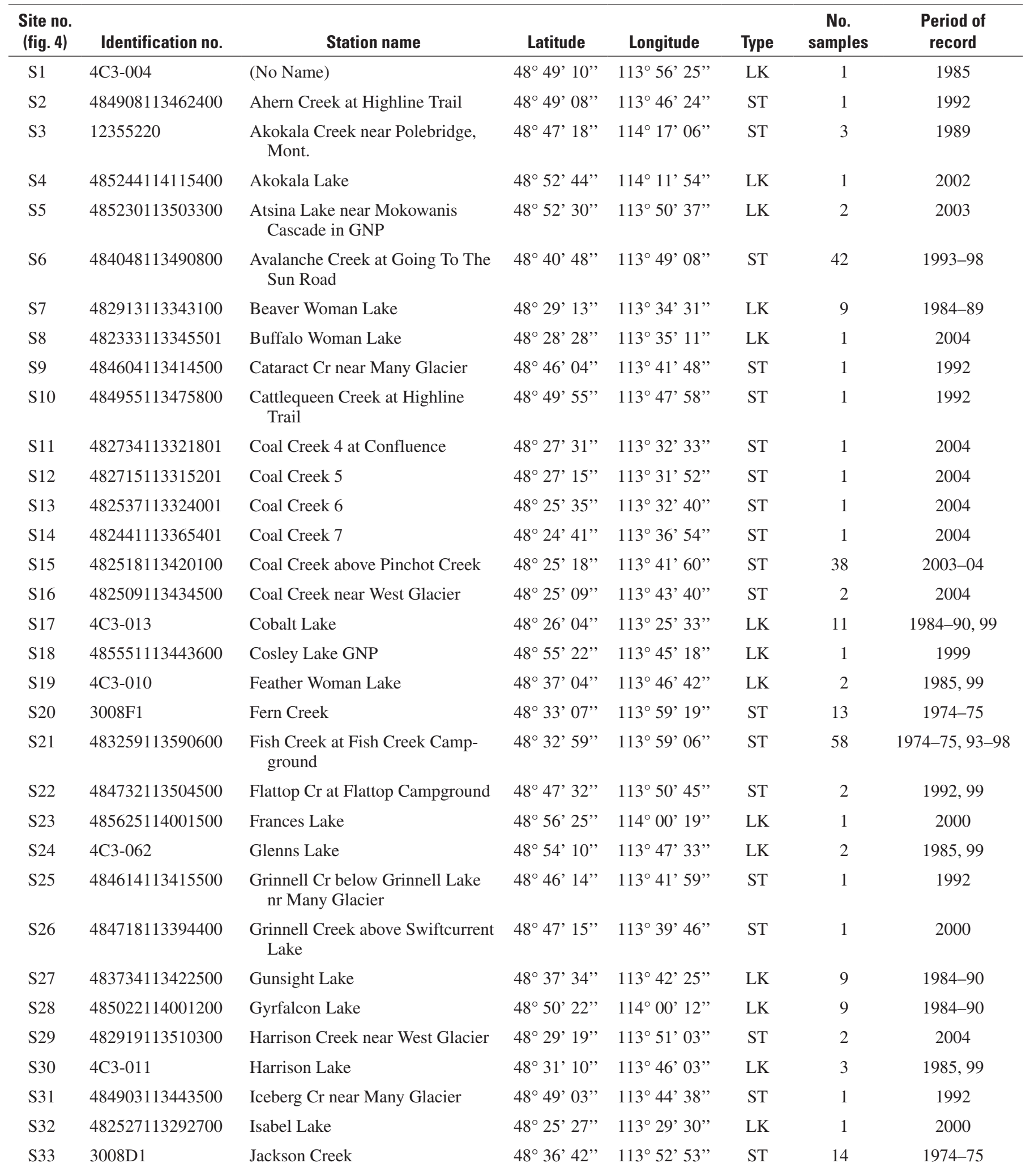


Table 6. Surface-water sampling sites in Glacier National Park, excluding sites on the North and Middle Forks of the Flathead River, Montana.-Continued

[no., number; LK, lake; ST, stream; GNP, Glacier National Park; Cr, Creek; ab, above; nr, near; identification numbers and station names from USEPA STORET and USGS NWIS]

\begin{tabular}{|c|c|c|c|c|c|c|c|}
\hline $\begin{array}{l}\text { Site no. } \\
\text { (fig. 4) }\end{array}$ & Identification no. & Station name & Latitude & Longitude & Type & $\begin{array}{l}\text { No. } \\
\text { samples }\end{array}$ & $\begin{array}{l}\text { Period of } \\
\text { record }\end{array}$ \\
\hline S34 & 485108113514200 & $\begin{array}{l}\text { Kootenai Creek at Fifty Mile } \\
\text { Camp }\end{array}$ & $48^{\circ} 51^{\prime} 08^{\prime \prime}$ & $113^{\circ} 51^{\prime} 42^{\prime \prime}$ & ST & 1 & 1992 \\
\hline S35 & 485639113563600 & $\begin{array}{l}\text { Lake Janet near Olson Mountain } \\
\text { in GNP }\end{array}$ & $48^{\circ} 56^{\prime} 39^{\prime \prime}$ & $113^{\circ} 56^{\prime} 39^{\prime \prime}$ & LK & 2 & 2003 \\
\hline S36 & 6320LA01 & Lake Sherburne & $48^{\circ} 49^{\prime} 35^{\prime \prime}$ & $113^{\circ} 31^{\prime} 27^{\prime \prime}$ & LK & 2 & 1980 \\
\hline S38 & 484154113435300 & Logan Creek at Headwater Spring & $48^{\circ} 41^{\prime} 54^{\prime \prime}$ & $113^{\circ} 43^{\prime} 53^{\prime \prime}$ & ST & 3 & 1994, 98 \\
\hline S39 & 484154113433000 & Logan Creek at Logan Pass & $48^{\circ} 41^{\prime} 54^{\prime \prime}$ & $113^{\circ} 43^{\prime} 30^{\prime \prime}$ & ST & 1 & 1994 \\
\hline $\mathrm{S} 40$ & 484325113455100 & $\begin{array}{l}\text { Logan Creek at The Snowshoe } \\
\text { Cabin }\end{array}$ & $48^{\circ} 43^{\prime} 25^{\prime \prime}$ & $113^{\circ} 45^{\prime} 51^{\prime \prime}$ & ST & 32 & 1993-98 \\
\hline $\mathrm{S} 44$ & 483133113595000 & $\begin{array}{l}\text { McDonald Cr below Lake } \\
\text { McDonald }\end{array}$ & $48^{\circ} 31^{\prime} 33^{\prime \prime}$ & $113^{\circ} 59^{\prime} 48^{\prime \prime}$ & ST & 14 & $1974-75$ \\
\hline $\mathrm{S} 45$ & 3008A2 & McDonald Creek & $48^{\circ} 38^{\prime} 08^{\prime \prime}$ & $113^{\circ} 51^{\prime} 59^{\prime \prime}$ & ST & 14 & $1974-75$ \\
\hline $\mathrm{S} 46$ & 484526113500700 & $\begin{array}{l}\text { McDonald Creek ab Confluence } \\
\text { with Mineral Cr }\end{array}$ & $48^{\circ} 45^{\prime} 26^{\prime \prime}$ & $113^{\circ} 50^{\prime} 07^{\prime \prime}$ & ST & 41 & 1993-98 \\
\hline S47 & 483828113512800 & $\begin{array}{l}\text { McDonald Creek at Bridge above } \\
\text { Lake McDonald }\end{array}$ & $48^{\circ} 38^{\prime} 28^{\prime \prime}$ & $113^{\circ} 51^{\prime} 28^{\prime \prime}$ & ST & 46 & 1993-98 \\
\hline $\mathrm{S} 48$ & 483133113594800 & $\begin{array}{l}\text { McDonald Creek at Bridge below } \\
\text { Lake McDonald }\end{array}$ & $48^{\circ} 31^{\prime} 33^{\prime \prime}$ & $113^{\circ} 59^{\prime} 48^{\prime \prime}$ & ST & 45 & 1993-98 \\
\hline S53 & 485038113493700 & Mineral Creek at Highline Trail & $48^{\circ} 50^{\prime} 38^{\prime \prime}$ & $113^{\circ} 49^{\prime} 37^{\prime \prime}$ & ST & 1 & 1992 \\
\hline S54 & 484530113491800 & $\begin{array}{l}\text { Mineral Creek at Foot Bridge near } \\
\text { Mouth }\end{array}$ & $48^{\circ} 45^{\prime} 30^{\prime \prime}$ & $113^{\circ} 49^{\prime} 18^{\prime \prime}$ & ST & 44 & 1992-98 \\
\hline S55 & 485245113485000 & $\begin{array}{l}\text { Mokowanis Lake in Glacier } \\
\text { National Park }\end{array}$ & $48^{\circ} 52^{\prime} 45^{\prime \prime}$ & $113^{\circ} 48^{\prime} 53^{\prime \prime}$ & LK & 3 & 1999,2003 \\
\hline S56 & 483237113271800 & Morning Star Lake & $48^{\circ} 32^{\prime} 37^{\prime \prime}$ & $113^{\circ} 27^{\prime} 22^{\prime \prime}$ & LK & 1 & 2000 \\
\hline S57 & 482154113390600 & Muir Creek near West Glacier & $48^{\circ} 21^{\prime} 57^{\prime \prime}$ & $113^{\circ} 39^{\prime} 06^{\prime \prime}$ & ST & 2 & 2004 \\
\hline S58 & 482844113265000 & Noname Lake & $48^{\circ} 28^{\prime} 44^{\prime \prime}$ & $113^{\circ} 26^{\prime} 50^{\prime \prime}$ & LK & 1 & 2002 \\
\hline S59 & 482946113411701 & Nyack Creek 2 & $48^{\circ} 29^{\prime} 46^{\prime \prime}$ & $113^{\circ} 41^{\prime} 17^{\prime \prime}$ & ST & 1 & 2004 \\
\hline S60 & 482720113480800 & Nyack Creek near West Glacier & $48^{\circ} 27^{\prime} 19^{\prime \prime}$ & $113^{\circ} 48^{\prime} 06^{\prime \prime}$ & ST & 2 & 2004 \\
\hline S61 & 483111113314701 & Nyack Creek 3, Upper Nyack & $48^{\circ} 31^{\prime} 11^{\prime \prime}$ & $113^{\circ} 31^{\prime} 47^{\prime \prime}$ & ST & 1 & 2004 \\
\hline S62 & 481656113355600 & Ole Creek near West Glacier & $48^{\circ} 16^{\prime} 54^{\prime \prime}$ & $113^{\circ} 35^{\prime} 59^{\prime \prime}$ & ST & 2 & 2004 \\
\hline S63 & 484300113355000 & Otokomi Lake & $48^{\circ} 43^{\prime} 00^{\prime \prime}$ & $113^{\circ} 35^{\prime} 50^{\prime \prime}$ & LK & 1 & 2002 \\
\hline
\end{tabular}


Table 6. Surface-water sampling sites in Glacier National Park, excluding sites on the North and Middle Forks of the Flathead River, Montana.-Continued

[no., number; LK, lake; ST, stream; GNP, Glacier National Park; Cr, Creek; ab, above; nr, near; identification numbers and station names from USEPA STORET and USGS NWIS]

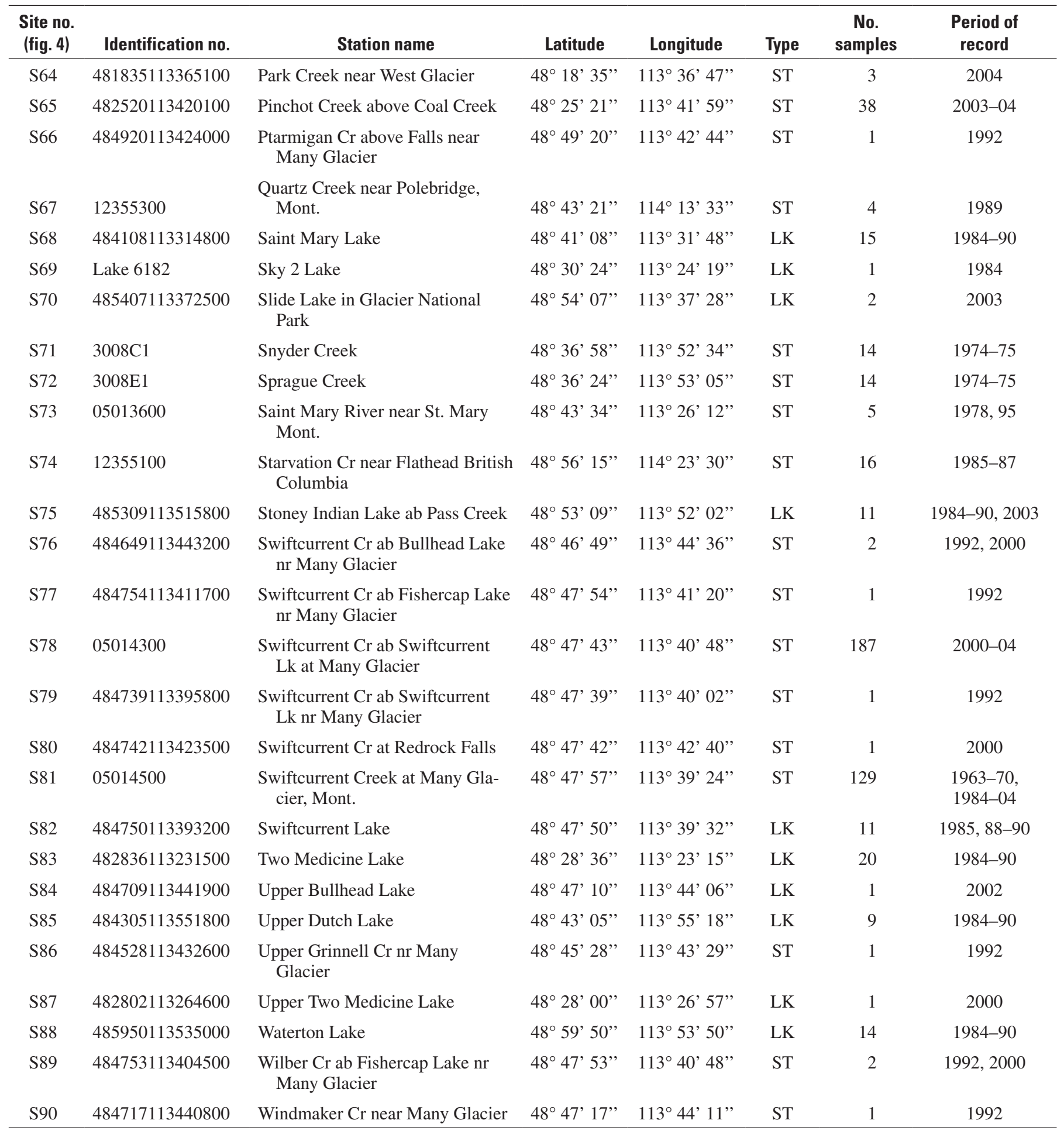




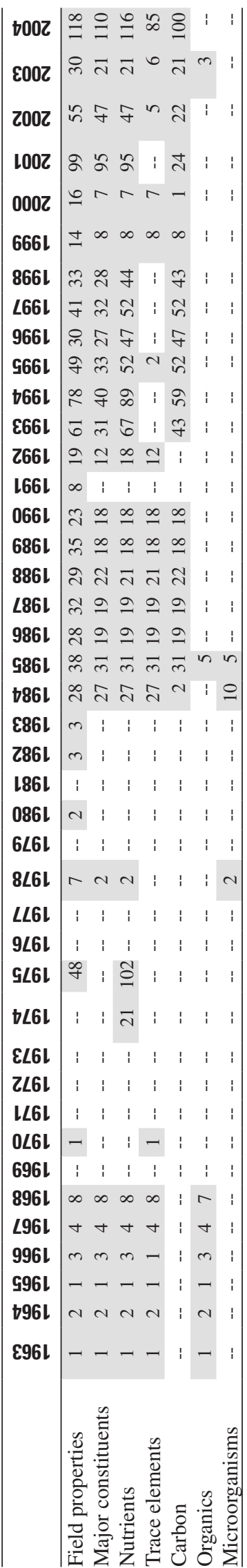

Canada as part of a network of sites in British Columbia to detect the existence of any significant trends in water quality (http://www.ec.gc.ca/water/en/manage/qual/e_keymap.htm, accessed November 2006). The MTDEQ collected samples at sites N3-N7 as part of a statewide water-quality monitoring network and the U.S. Forest Service sampled site N7. On the Middle Fork, the USGS collected water-quality samples at sites M1 and M6, the MTDEQ collected samples at all eight Middle Fork sites, and the U.S. Forest Service collected samples at sites M1 and M7. The ROMN database also contains major-constituent and trace-element data for 331 sites sampled in 1976 and 1978 as part of the NURE program (http://pubs. usgs.gov/of/1997/ofr-97-0492/, accessed November 2006). Because of the age (more than 25 years old) and unknown quality of NURE data and the availability of more recent datasets, results for NURE sites in GLAC are not discussed as part of this report.

Surface water in GLAC is fairly dilute and moderately buffered. Specific conductance ranged from 6.1 to $178 \mu \mathrm{S} / \mathrm{cm}$, and alkalinity ranged from 2.0 to $93 \mathrm{mg} / \mathrm{L}$ (table 9). The dominant anion in surface water was bicarbonate (based on $\mathrm{pH}$ and alkalinity), and the dominant cations were calcium (median 14 $\mathrm{mg} / \mathrm{L}$ ) and magnesium (median $4.0 \mathrm{mg} / \mathrm{L}$ ). The predominance of these constituents is attributed to weathering of carbonate minerals in the Proterozoic sedimentary rocks. Other major anions were sulfate, which ranged from 0.16 to $9.2 \mathrm{mg} / \mathrm{L}$, and chloride, which ranged from less than 0.01 to $3.7 \mathrm{mg} / \mathrm{L}$. The relatively low chloride concentrations (median $0.1 \mathrm{mg} / \mathrm{L}$ ) indicate that atmospheric deposition probably is the dominant source of chloride in surface water (Clark and others, 1999). Although considerably less abundant than bicarbonate, sulfate concentrations were substantially higher (median $2.3 \mathrm{mg} / \mathrm{L}$ ) than chloride, indicating weathering of sedimentary rocks also likely contributes sulfate to surface water. Summaries of water quality for the main stems of the North Fork and Middle Fork of the Flathead River are shown separately in table 10. These two rivers have much larger drainage areas than streams within the park boundary and, in addition, have a large percentage of their drainage area outside the park boundary. The ratio of major constituents in river-water samples was similar to samples collected from streams and lakes within the park, although major-constituent concentrations were up to three times higher in the North and Middle Forks of the Flathead River.

The most temporally intensive dataset available in the park is for Swiftcurrent Creek above Swiftcurrent Lake (S78). This site has been sampled at least monthly, and as frequent as weekly during snowmelt, since 2001 as part of the USGS Hydrologic Benchmark Network. Seasonal variations in alkalinity, calcium, and sulfate concentrations at S78 are shown in figure 8 for 2001 through 2004. Major constituent concentrations decrease in spring and reach a minimum in late May or early June at peak snowmelt discharge. Concentrations increase steadily through the summer and fall as snowmelt declines and ground water provides a greater percentage of streamflow. Although major constituents followed the same 
Table 8. Water-quality sampling sites on the North Fork and Middle Fork of the Flathead River in Glacier National Park, Montana.

[no., number; ab, above; nr, near]

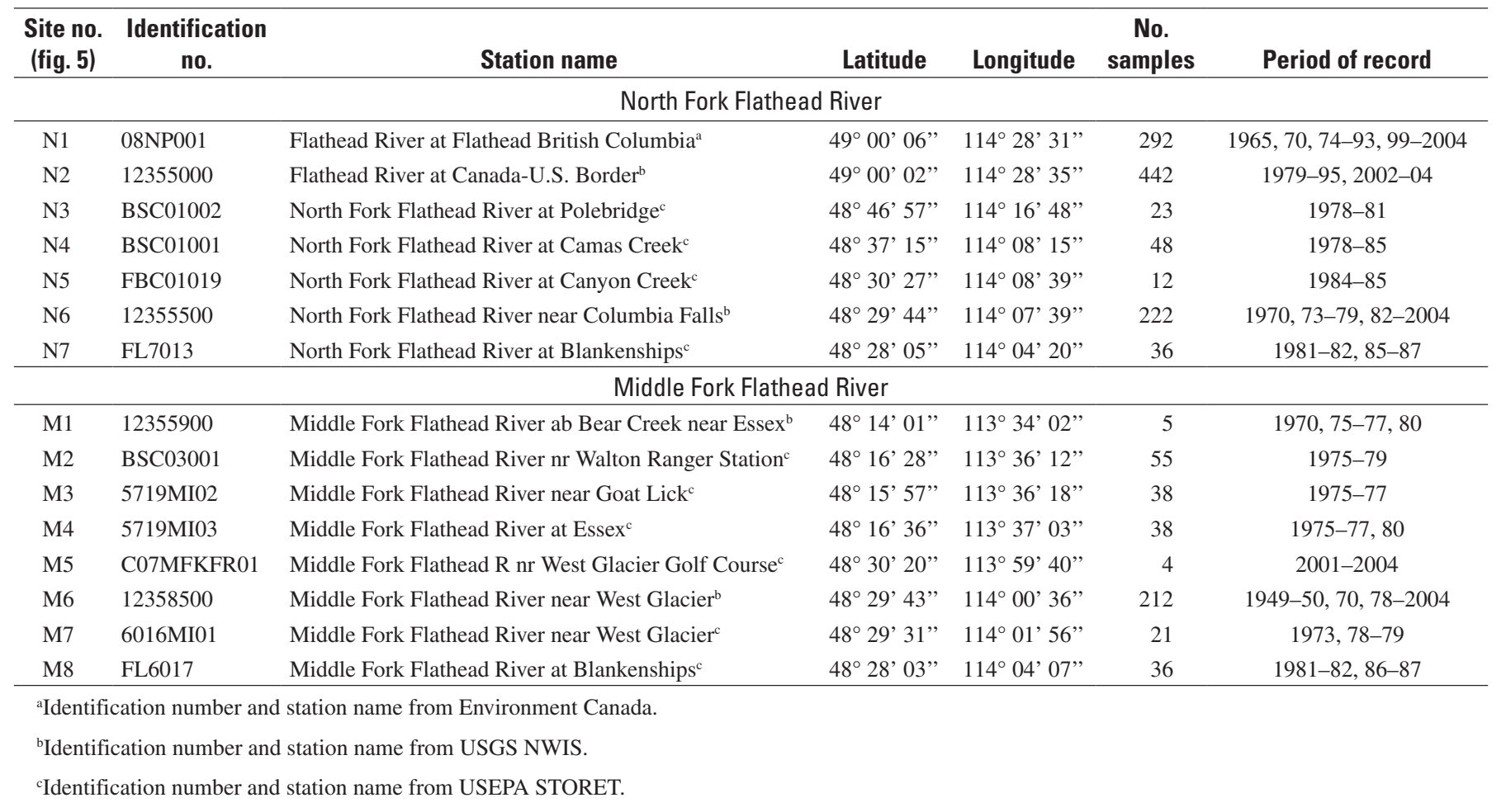

general pattern, there were some subtle differences in seasonal patterns. Alkalinity and calcium increased slightly in March at the start of the snowmelt period, then declined to their minimum concentrations in late April. In contrast, sulfate did not show an increase in March, and concentrations reached their minimum a few weeks later in June. These subtle variations likely reflect differences in weathering and biological processes that control surface-water quality.

General spatial patterns in water quality are illustrated by comparing lakes and streams in GLAC to the North Fork and Middle Fork of the Flathead River (fig. 9). pH generally was between 7.5 and 8.5 and fairly uniform among surface-water types, reflecting the predominance of carbonate minerals in the bedrock and buffering of surface waters by the carbonate system. Specific conductance, which is proportional to dissolved solids, was higher at the mainstem sites compared to lakes and streams in the park. This reflects longer hydrologic flow paths and greater opportunity for rock-water interactions in the larger river basins. Differences in geology and landuse activities also may be important factors. The most dilute surface waters were small lakes (less than $0.05 \mathrm{~km}^{2}$ ) situated at high elevations (above 1,500 m). Of the sampled lakes in the park, the most dilute were sites S17, S19, S42, and S85, which had specific conductance values less than $10 \mu \mathrm{S} / \mathrm{cm}$ and $\mathrm{pH}$ values below 7.0. They also had alkalinities below $10 \mathrm{mg} / \mathrm{L}$ indicating some lakes in the park may be sensitive to acidification by deposition of atmospheric contaminants. However, less than 5 percent of lakes in the park have been sampled so the population of sensitive lakes is not well defined. Research is currently (2007) underway to identify and quantify the extent of sensitive lakes in GLAC through application of statistical models based on watershed characteristics (Nanus, 2005; Nanus and others, 2005).

Nutrients measured in streams and lakes inside GLAC include ammonia, nitrate, nitrite, organic nitrogen, orthophosphate, and dissolved and total phosphorus (table 9). Concentrations of most nutrients were close to or below detection with the exception of nitrate, which was detected in nearly all samples in concentrations ranging from less than 0.001 to 1.2 $\mathrm{mg} / \mathrm{L}$. Concentrations at some sites were higher than expected considering that atmospheric deposition is the primary source of inorganic nitrogen to surface-water systems in GLAC (Clark and others, 1999). Some of the highest nitrate concentrations $(0.12$ to $0.84 \mathrm{mg} / \mathrm{L})$ were measured in Coal Creek above Pinchot Creek (S15) during the 2004 snowmelt period. Elevated nitrate at this site is attributed to leaching of nitrogen from soil and ash following a large wildfire that burned more than 80 percent of the drainage basin in 2003. Post-fire leaching of nutrients to surface water, particularly nitrate, is a commonly observed response in the first or second year following a fire (Ranalli, 2004). Elevated nitrate (range 0.034 to $1.20 \mathrm{mg} / \mathrm{L}$ ) also was reported at Logan Creek at the Snowshoe Cabin (S40), although this drainage was not affected by recent wildfires. Hauer and others (2007) suggested that elevated nitrate may be caused by alders, which increase the nitrogen content of soil through symbiotic nitrogen fixation. The 
Table 9. Summary of selected water-quality data for surface-water sites in Glacier National Park excluding the Middle and North Forks of the Flathead River, Montana, 1980 to 2004.

[no. number; chronic aquatic-life (and human-health) standards from www.deq.state.mt.us/wqinfo/Standards/Index.asp; <, less than; >, greater than; --, not reported; ${ }^{\circ} \mathrm{C}$, degrees Celsius; $\mathrm{mg} / \mathrm{L}$, milligrams per liter; $\mu \mathrm{S} / \mathrm{cm}$, microsiemens per centimeter at $25{ }^{\circ} \mathrm{C} ; \mathrm{CaCO}_{3}$, calcium carbonate; $\mathrm{N}$, nitrogen; $\mathrm{P}$, phosphorous; $\mu \mathrm{g} / \mathrm{L}$, micrograms per liter; some nutrients and trace elements have multiple reporting limits]

\begin{tabular}{|c|c|c|c|c|c|c|}
\hline Constituent or property & $\begin{array}{l}\text { No. } \\
\text { sites }\end{array}$ & $\begin{array}{l}\text { No. analyses } \\
\text { (no. censored) }\end{array}$ & $\begin{array}{l}\text { Minimum } \\
\text { value }\end{array}$ & $\begin{array}{c}\text { Median } \\
\text { value }\end{array}$ & $\begin{array}{l}\text { Maximum } \\
\text { value }\end{array}$ & $\begin{array}{c}\text { Aquatic-life } \\
\text { (human-health) } \\
\text { standard }\end{array}$ \\
\hline \multicolumn{7}{|c|}{ Field properties } \\
\hline Temperature, water $\left({ }^{\circ} \mathrm{C}\right)$ & 44 & 235 & 0.0 & 7.0 & 19.0 & $<19.4$ \\
\hline Oxygen, dissolved (mg/L) & 0 & 0 & -- & -- & -- & $>4.0$ \\
\hline $\mathrm{pH}$ (standard units) & 63 & 457 & 5.54 & 7.93 & 8.66 & a $6.5-9.0$ \\
\hline Specific conductance $(\mu \mathrm{S} / \mathrm{cm})$ & 55 & 576 & 6.1 & 101 & 178 & -- \\
\hline \multicolumn{7}{|c|}{ Major constituents } \\
\hline Alkalinity $\left(\mathrm{mg} / \mathrm{L}\right.$ as $\left.\mathrm{CaCO}_{3}\right)$ & 63 & 457 & 2.0 & 49 & 93 & -- \\
\hline Calcium, dissolved (mg/L) & 58 & $444(1)$ & $<.1$ & 14 & 25 & -- \\
\hline Chloride, dissolved (mg/L) & 59 & 454 (117) & $<.01$ & .1 & 3.7 & -- \\
\hline Fluoride, dissolved (mg/L) & 30 & $128(110)$ & ${ }^{\mathrm{b}} .01$ & $<.2$ & .31 & (4) \\
\hline Magnesium, dissolved (mg/L) & 59 & $451(1)$ & $<.1$ & 4.0 & 10 & -- \\
\hline Potassium, dissolved (mg/L) & 59 & $452(34)$ & ${ }^{\mathrm{b}} .03$ & .16 & .42 & -- \\
\hline Silica, dissolved (mg/L) & 59 & $447(2)$ & b. 20 & 2.2 & 7.6 & -- \\
\hline Sodium, dissolved (mg/L) & 59 & $453(5)$ & ${ }^{\mathrm{b}} .02$ & .46 & 1.3 & -- \\
\hline Sulfate, dissolved (mg/L) & 58 & $449(3)$ & b. 16 & 2.3 & 9.2 & -- \\
\hline \multicolumn{7}{|c|}{ Nutrients and carbon } \\
\hline Ammonia, dissolved (mg/L as $\mathrm{N})$ & 65 & $650(361)$ & ${ }^{\mathrm{b}} 0.001$ & 0.006 & 0.094 & $\mathrm{c} 2.8$ \\
\hline Nitrate, dissolved (mg/L as $\mathrm{N}$ ) & 73 & $808(16)$ & $<.001$ & .12 & 1.2 & $(10)$ \\
\hline Nitrite, dissolved (mg/L as $\mathrm{N})$ & 17 & $94(27)$ & $<.0001$ & .0003 & .002 & $(1.0)$ \\
\hline Organic nitrogen, dissolved ( $\mathrm{mg} / \mathrm{L}$ as $\mathrm{N})$ & 14 & 75 & .018 & .057 & .20 & -- \\
\hline Orthophosphate, dissolved (mg/L as $\mathrm{P})$ & 41 & $513(136)$ & b.0003 & .0009 & .01 & -- \\
\hline Phosphorus, dissolved (mg/L as $\mathrm{P}$ ) & 14 & $144(36)$ & $<.0006$ & .0014 & .007 & -- \\
\hline Phosphorus, total (mg/L as P) & 46 & $581(54)$ & b.0009 & .0038 & .35 & -- \\
\hline Carbon, dissolved organic (mg/L) & 53 & 514 & .06 & .87 & 6.4 & -- \\
\hline \multicolumn{7}{|c|}{ Trace elements } \\
\hline Aluminum, dissolved $(\mu \mathrm{g} / \mathrm{L})$ & 14 & 141 & 1 & 14 & 96 & 87 \\
\hline Arsenic, dissolved $(\mu \mathrm{g} / \mathrm{L})$ & 13 & $90(90)$ & $<1$ & $<1$ & $<1$ & ${ }^{\mathrm{d}} 150(10)$ \\
\hline Barium, dissolved ( $\mu \mathrm{g} / \mathrm{L})$ & 15 & $93(1)$ & $<1$ & 52 & 119 & $(2,000)$ \\
\hline Cobalt, dissolved ( $\mu \mathrm{g} / \mathrm{L})$ & 14 & $91(91)$ & $<1$ & $<1$ & $<3$ & -- \\
\hline Copper, dissolved $(\mu \mathrm{g} / \mathrm{L})$ & 14 & $79(76)$ & $<1$ & $<1$ & 2.8 & $\mathrm{c,d} 5.2(1,300)$ \\
\hline Iron, dissolved ( $\mu \mathrm{g} / \mathrm{L})$ & 26 & $161(101)$ & $<1$ & $<100$ & $<100$ & d $1,000\left({ }^{e} 300\right)$ \\
\hline Lead, dissolved $(\mu \mathrm{g} / \mathrm{L})$ & 13 & $82(60)$ & $<1$ & $<1$ & 2 & ${ }^{\mathrm{c}, \mathrm{d}} 1.3(15)$ \\
\hline Manganese, dissolved ( $\mu \mathrm{g} / \mathrm{L})$ & 17 & $18(9)$ & $<1$ & 1 & 4 & $\left({ }^{e} 50\right)$ \\
\hline Mercury, dissolved ( $\mu \mathrm{g} / \mathrm{L})$ & 13 & $65(65)$ & $<1$ & $<1$ & $<1$ & ${ }^{\mathrm{d}} 0.91(.05)$ \\
\hline Molybdenum, dissolved ( $\mu \mathrm{g} / \mathrm{L})$ & 14 & $129(126)$ & $<1$ & $<1$ & $<10$ & -- \\
\hline Selenium, dissolved ( $\mu \mathrm{g} / \mathrm{L})$ & 13 & $65(65)$ & $<1$ & $<1$ & $<1$ & d5 (50) \\
\hline
\end{tabular}

${ }^{\text {a }}$ Natural $\mathrm{pH}$ outside this range must be maintained without change.

${ }^{b}$ Minimum reported value less than minimum censored value.

${ }^{\mathrm{c}}$ Table value standard calculated for a hardness of $50 \mathrm{mg} / \mathrm{L}$ for trace elements or $\mathrm{pH}$ of 7.9 at $20^{\circ} \mathrm{C}$ for ammonia.

'Total recoverable concentration.

${ }^{\mathrm{e} S e c o n d a r y ~ s t a n d a r d ~ b a s e d ~ a e s t h e t i c ~ p r o p e r t i e s ~ s u c h ~ a s ~ t a s t e, ~ o d o r, ~ a n d ~ s t a i n i n g . ~}$ 
Table 10. Summary of selected water-quality data for sampling sites on the North Fork and Middle Fork of the Flathead River in Glacier National Park, Montana, 1980 to 2004.

[no. number; chronic aquatic-life (and human-health) standards from www.deq.state.mt.us/wqinfo/Standards/Index.asp; <, less than; >, greater than; --, not reported; ${ }^{\circ} \mathrm{C}$, degrees Celsius; $\mathrm{mg} / \mathrm{L}$, milligrams per liter; $\mu \mathrm{S} / \mathrm{cm}$, microsiemens per centimeter at $25^{\circ} \mathrm{C} ; \mathrm{CaCO}_{3}$, calcium carbonate; $\mathrm{N}$, nitrogen; $\mathrm{P}$, phosphorous; $\mathrm{col} / 100 \mathrm{~mL}$, colonies per 100 milliliters; $\mu \mathrm{g} / \mathrm{L}$, micrograms per liter; some trace elements have multiple reporting limits]

\begin{tabular}{|c|c|c|c|c|c|c|c|}
\hline Constituent or property & River Section & $\begin{array}{l}\text { No. } \\
\text { sites }\end{array}$ & $\begin{array}{l}\text { No. analyses } \\
\text { (no. censored) }\end{array}$ & $\begin{array}{c}\text { Minimum } \\
\text { value }\end{array}$ & $\begin{array}{c}\text { Median } \\
\text { value }\end{array}$ & $\begin{array}{c}\text { Maximum } \\
\text { value }\end{array}$ & $\begin{array}{c}\text { Aquatic-life } \\
\text { (human-health) } \\
\text { standard }\end{array}$ \\
\hline \multicolumn{8}{|c|}{ Field properties } \\
\hline Temperature, water $\left({ }^{\circ} \mathrm{C}\right)$ & Middle Fork & 4 & 194 & .0 & 7.5 & 18.2 & \\
\hline Oxygen, dissolved (mg/L) & North Fork & 3 & 167 & 8.4 & 10.2 & 14.3 & $>4.0$ \\
\hline $\mathrm{pH}$ (standard units) & Middle Fork & 3 & 29 & 6.93 & 8.10 & 8.60 & \\
\hline \multirow{2}{*}{ Specific conductance $(\mu \mathrm{S} / \mathrm{cm})$} & North Fork & 3 & 523 & 138 & 251 & 310 & -- \\
\hline & Middle Fork & 4 & 195 & 120 & 172 & 273 & \\
\hline \multicolumn{8}{|c|}{ Major constituents } \\
\hline \multirow{2}{*}{ Alkalinity $\left(\mathrm{mg} / \mathrm{L}\right.$ as $\left.\mathrm{CaCO}_{3}\right)$} & North Fork & 3 & 479 & 76 & 134 & 154 & -- \\
\hline & Middle Fork & 1 & 3 & 106 & 109 & 114 & \\
\hline Chloride, dissolved (mg/L) & Middle Fork & 2 & $13(3)$ & b. .07 & .2 & .9 & \\
\hline \multirow{2}{*}{ Fluoride, dissolved (mg/L) } & North Fork & 3 & $518(14)$ & b. .02 & .08 & 1.5 & (4) \\
\hline & Middle Fork & 2 & $13(10)$ & ${ }^{\mathrm{b}} .04$ & $<.1$ & .2 & \\
\hline \multirow[t]{2}{*}{ Magnesium, dissolved (mg/L) } & North Fork & 6 & 394 & 2.9 & 8.5 & 12 & -- \\
\hline & Middle Fork & 3 & 25 & 4.1 & 6.1 & 9.1 & \\
\hline \multirow[t]{2}{*}{ Potassium, dissolved (mg/L) } & North Fork & 6 & 370 & .2 & .3 & .9 & -- \\
\hline & Middle Fork & 3 & 25 & .2 & .3 & 1.6 & \\
\hline \multirow{2}{*}{ Silica, dissolved (mg/L) } & North Fork & 4 & 195 & 1.5 & 2.6 & 5.7 & -- \\
\hline & Middle Fork & 2 & 13 & 3.3 & 4.0 & 5.3 & \\
\hline \multirow[t]{2}{*}{ Sodium, dissolved (mg/L) } & North Fork & 6 & 374 & .4 & .9 & 7.0 & -- \\
\hline & Middle Fork & 3 & 25 & .5 & .8 & 9.9 & \\
\hline \multirow[t]{2}{*}{ Nitrite, dissolved (mg/L as N) } & North Fork & 4 & 107 (97) & $<.001$ & $<.005$ & .08 & (1.0) \\
\hline & Middle Fork & 1 & $20(16)$ & $<.001$ &.$<002$ & .01 & \\
\hline \multirow[t]{2}{*}{ Orthophosphate, dissolved (mg/L as P) } & North Fork & 3 & $116(105)$ & $<.001$ & $<.003$ & .024 & -- \\
\hline & Middle Fork & 1 & $20(16)$ & $<.001$ & $<.007$ & .007 & \\
\hline \multirow[t]{2}{*}{ Phosphorus, dissolved (mg/L as P) } & North Fork & 5 & $111(87)$ & $<.001$ & $<.002$ & .020 & -- \\
\hline & Middle Fork & 2 & 8 & .001 & .005 & .014 & \\
\hline Phosphorus, total (mg/L as P) & North Fork & 7 & $579(107)$ & $<.001$ & .006 & .42 & -- \\
\hline & Middle Fork & 2 & $31(5)$ & b.001 & .008 & .20 & \\
\hline Carbon, dissolved organic (mg/L) & North Fork & 5 & 42 & .0 & 1.3 & 7.0 & -- \\
\hline & Middle Fork & 2 & 10 & .04 & .9 & 2.0 & \\
\hline Fecal coliform (col/100 mL) & North Fork & 2 & $94(5)$ & $<1$ & 3 & 120 & -- \\
\hline & Middle Fork & 1 & $24(10)$ & $<1$ & 1 & 75 & \\
\hline Fecal strep (col/100 mL) & North Fork & 2 & 94 & 1 & 3 & 170 & -- \\
\hline & Middle Fork & 1 & 24 & 1 & 3 & 93 & \\
\hline
\end{tabular}


Table 10. Summary of selected water-quality data for sampling sites on the North Fork and Middle Fork of the Flathead River in Glacier National Park, Montana, 1980 to 2004.-Continued

[no. number; chronic aquatic-life (and human-health) standards from www.deq.state.mt.us/wqinfo/Standards/Index.asp; <, less than; >, greater than; --, not reported; ${ }^{\circ} \mathrm{C}$, degrees Celsius; mg/L, milligrams per liter; $\mu \mathrm{S} / \mathrm{cm}$, microsiemens per centimeter at $25^{\circ} \mathrm{C} ; \mathrm{CaCO}_{3}$, calcium carbonate; $\mathrm{N}$, nitrogen; $\mathrm{P}$, phosphorous; $\mathrm{col} / 100 \mathrm{~mL}$, colonies per 100 milliliters; $\mu \mathrm{g} / \mathrm{L}$, micrograms per liter; some trace elements have multiple reporting limits]

\begin{tabular}{|c|c|c|c|c|c|c|c|}
\hline Constituent or property & River Section & $\begin{array}{l}\text { No. } \\
\text { sites }\end{array}$ & $\begin{array}{l}\text { No. analyses } \\
\text { (no. censored) }\end{array}$ & $\begin{array}{l}\text { Minimum } \\
\text { value }\end{array}$ & $\begin{array}{c}\text { Median } \\
\text { value }\end{array}$ & $\begin{array}{c}\text { Maximum } \\
\text { value }\end{array}$ & $\begin{array}{c}\text { Aquatic-life } \\
\text { (human-health) } \\
\text { standard }\end{array}$ \\
\hline \multicolumn{8}{|c|}{ Trace elements } \\
\hline \multirow[t]{2}{*}{ Aluminum, total $(\mu \mathrm{g} / \mathrm{L})$} & North Fork & 1 & $154(1)$ & $<0.2$ & 33 & 4,000 & d87 \\
\hline & Middle Fork & 0 & 0 & -- & -- & -- & \\
\hline \multirow[t]{2}{*}{ Arsenic, total $(\mu \mathrm{g} / \mathrm{L})$} & North Fork & 3 & $421(21)$ & $<.1$ & .3 & 2.6 & $150\left({ }^{\mathrm{d}} 10\right)$ \\
\hline & Middle Fork & 2 & $11(11)$ & $<1$ & $<2$ & $<2.6$ & \\
\hline \multirow{2}{*}{ Barium, total $(\mu \mathrm{g} / \mathrm{L})$} & North Fork & 2 & $165(1)$ & b24 & 55 & 223 & $\left({ }^{d} 2,000\right)$ \\
\hline & Middle Fork & 0 & 0 & -- & -- & -- & \\
\hline \multirow[t]{2}{*}{ Cadmium, total ( $\mu \mathrm{g} / \mathrm{L})$} & North Fork & 3 & $191(127)$ & b.004 & $<.1$ & 6 & ${ }^{\mathrm{c}} 0.2\left({ }^{\mathrm{d}} 5\right)$ \\
\hline & Middle Fork & 2 & $11(10)$ & $<.1$ & $<.11$ & .14 & \\
\hline \multirow[t]{2}{*}{ Chromium, total $(\mu \mathrm{g} / \mathrm{L})$} & North Fork & 3 & $150(30)$ & b. 18 & .4 & 10 & ${ }^{\mathrm{c}} 68\left({ }^{\mathrm{d}} 100\right)$ \\
\hline & Middle Fork & 1 & $12(8)$ & $<.8$ & $<1$ & 4.4 & \\
\hline \multirow[t]{2}{*}{ Cobalt, total $\mu \mathrm{g} / \mathrm{L})$} & North Fork & 2 & $165(29)$ & ${ }^{\mathrm{b}} .008$ & .1 & 2.3 & -- \\
\hline & Middle Fork & 0 & 0 & -- & -- & -- & \\
\hline \multirow[t]{2}{*}{ Copper, total $(\mu \mathrm{g} / \mathrm{L})$} & North Fork & 3 & $211(30)$ & b. 1 & 1 & 12 & c7.3 ( $\left.{ }^{\mathrm{d}} 1,300\right)$ \\
\hline & Middle Fork & 1 & $10(3)$ & $<.6$ & 1.9 & 12 & \\
\hline \multirow[t]{2}{*}{ Iron, total $(\mu \mathrm{g} / \mathrm{L})$} & North Fork & 2 & 401 & 4.5 & 42 & 5,960 & $1,000(\mathrm{~d}, \mathrm{e} 300)$ \\
\hline & Middle Fork & 1 & 4 & 10 & 25 & 40 & \\
\hline \multirow[t]{2}{*}{ Lead, total $(\mu \mathrm{g} / \mathrm{L})$} & North Fork & 3 & $211(128)$ & $<.005$ & $<.2$ & 57 & ${ }^{\mathrm{c}} 2.2\left({ }^{\mathrm{d}} 15\right)$ \\
\hline & Middle Fork & 1 & $10(6)$ & b. .3 & $<1$ & 4.2 & \\
\hline \multirow[t]{2}{*}{ Manganese, total ( $\mu \mathrm{g} / \mathrm{L})$} & North Fork & 2 & $422(88)$ & ${ }^{\mathrm{b}} .06$ & 5 & 180 & $(\mathrm{~d}, \mathrm{e} 50)$ \\
\hline & Middle Fork & 0 & 0 & -- & -- & -- & \\
\hline \multirow[t]{2}{*}{ Molybdenum, total ( $\mu \mathrm{g} / \mathrm{L})$} & North Fork & 1 & $154(1)$ & $<.1$ & .3 & 1.1 & -- \\
\hline & Middle Fork & 0 & 0 & -- & -- & -- & \\
\hline \multirow[t]{2}{*}{ Nickel, total $(\mu \mathrm{g} / \mathrm{L})$} & North Fork & 3 & $240(102)$ & $<.05$ & .4 & 8 & $\mathrm{c} 41$ \\
\hline & Middle Fork & 1 & $10(6)$ & b. 38 & $<1$ & 6.3 & \\
\hline \multirow[t]{2}{*}{ Selenium, total $(\mu \mathrm{g} / \mathrm{L})$} & North Fork & 3 & $387(50)$ & $<.05$ & .2 & 1 & $5\left({ }^{d} 50\right)$ \\
\hline & Middle Fork & 1 & 1 & 0 & -- & -- & \\
\hline \multirow[t]{2}{*}{ Zinc, total $(\mu \mathrm{g} / \mathrm{L})$} & North Fork & 3 & $211(29)$ & $<.05$ & 1.9 & 211 & ${ }^{\mathrm{c}} 94\left({ }^{\mathrm{d}} 2,000\right)$ \\
\hline & Middle Fork & 1 & $10(5)$ & $<1$ & 2.3 & $<40$ & \\
\hline
\end{tabular}

${ }^{\text {aNatural }} \mathrm{pH}$ outside this range must be maintained without change.

${ }^{\mathrm{b}}$ Minimum reported value less than minimum censored value.

'Table value standard calculated for a hardness of $75 \mathrm{mg} / \mathrm{L}$ for trace elements or $\mathrm{pH}$ of 7.1 at $20{ }^{\circ} \mathrm{C}$ for ammonia.

${ }^{\mathrm{d}}$ Dissolved concentration.

eSecondary standard based aesthetic properties such as taste, odor, and staining.

Logan Creek drainage has a particularly high density of alders because the landscape is dominated by steep avalanche terrain (Hauer and others, 2007). Summaries of nutrient concentrations for mainstem sites on the North and Middle Forks of the Flathead River are shown separately in table 10. Nutrient concentrations generally were low and were similar between the two forks of the river.

Processes controlling seasonal and spatial patterns in surface-water nutrients can be inferred from a few datasets available for the park. Frequent sampling at Swiftcurrent Creek above Swiftcurrent Lake (S78) on the east side of the park reveals a complicated seasonal pattern in stream-water nitrate (fig. 8). Concentrations increase rapidly during snowmelt in April and May due to preferential release of nitrate from the snowpack in addition to flushing of soils by infiltrating melt water. As melting subsides, stream-water concentrations begin to decline and reach a minimum in August near the end of the growing season. Nitrate concentrations increase through the winter probably because of greater contributions of nutrientenriched ground water to streamflow coupled with decreasing biological demand during snow-covered months. The cause of the slight decline in nitrate during March before snowmelt is not clear, but might be related to early season breakup of ice cover on streams. 

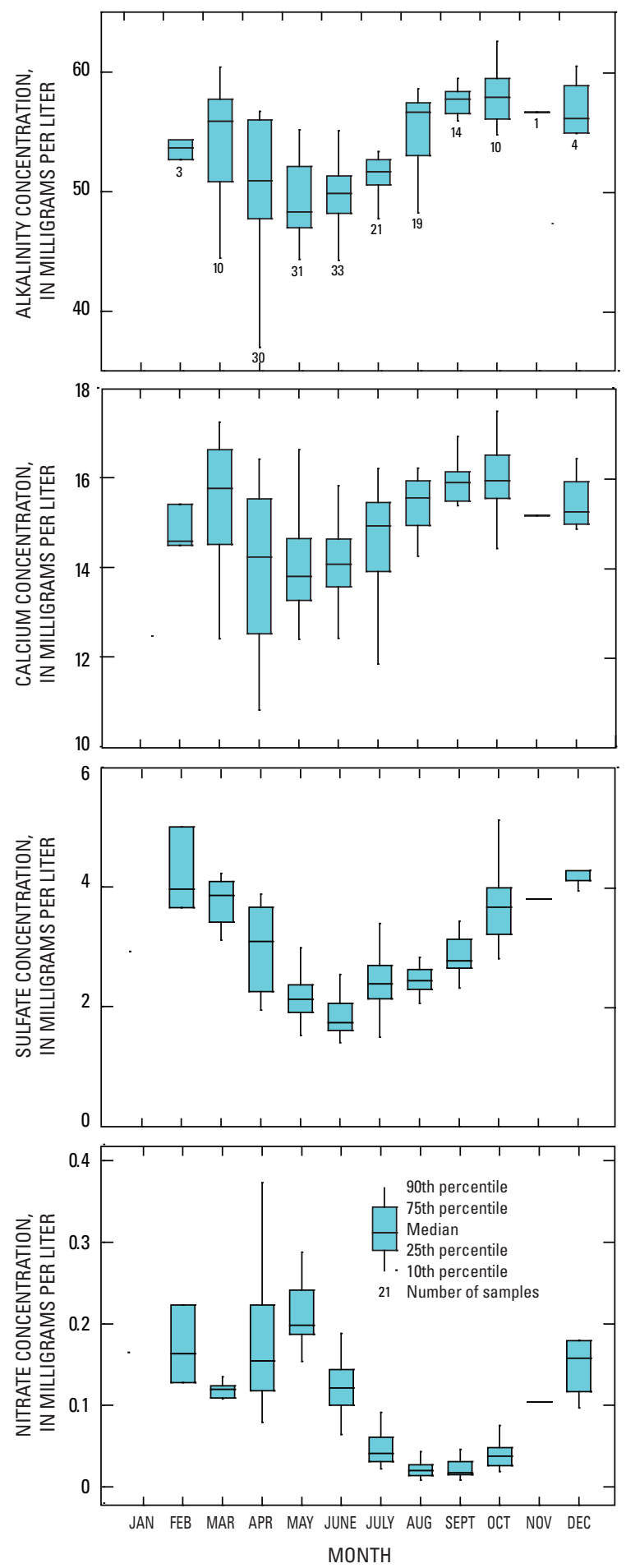

Figure 8. Seasonal variation in water quality at Swiftcurrent Creek above Swiftcurrent Lake at Many Glacier (S78) in Glacier National Park, Montana, 2001-2004.
Some general spatial patterns are apparent in a comparison of nutrients among streams and lakes in the park and the Middle and North Forks of the Flathead River (fig. 9). Orthophosphate was elevated in the North Fork and Middle Forks compared to lakes and streams in the park. Because phosphorus typically is associated with sediment, the higher concentrations probably reflect greater sediment loads in the larger river systems. This explanation is reasonable considering timber harvesting and road network land-use activities in areas outside the park boundary. Two west-side tributaries to the North Fork, Big Creek and Coal Creek, were 303(d) listed in 2006 as impaired for aquatic life and cold water fishery because of sedimentation from forest road networks and timber harvesting (http://www.deq.state.mt.us/CWAIC/default. aspx). By contrast, nitrate was lowest in the North Fork of the Flathead River, perhaps reflecting greater capacity of soils and forests to utilized atmospheric nitrogen in this drainage. Nitrate was elevated in park streams relative to lakes, partly reflecting the elevated concentrations in Coal Creek above Pinchot Creek (S15) and Logan Creek at Snowshoe Cabin (S40) discussed previously. Nitrate concentrations in park lakes ranged from less than 0.001 to $0.2 \mathrm{mg} / \mathrm{L}$, and most of the values above $0.1 \mathrm{mg} / \mathrm{L}$ were measured in Lake McDonald, which has lake shore developments and is used intensively by visitors (Ellis and others. 1992). Concentrations in highelevation lakes were low (less than $0.1 \mathrm{mg} / \mathrm{L}$ ) throughout the park indicating rates of atmospheric nitrogen deposition have not yet exceeded biological uptake. There is concern, however, that increased deposition of nitrogen to high-elevation aquatic ecosystems could eventually result in decreases in lake clarity, episodic acidification of surface water, and changes in species composition of aquatic organisms (Porter and others, 2005).

Most high-elevation lakes and streams in GLAC have physical characteristics that make them sensitive to increases in atmospheric deposition of nitrogen (Burns, 2004).

Concentrations for 11 trace elements were reported for lakes and streams sampled inside the park. At most of these sites only dissolved trace-element concentrations were measured; summaries are presented in table 9. The majority of concentrations were less than laboratory reporting levels and only aluminum and barium had a substantial number of detected concentrations above $10 \mu \mathrm{g} / \mathrm{L}$. Aluminum concentrations ranged from 1 to $96 \mu \mathrm{g} / \mathrm{L}$ and were somewhat higher than expected given the neutral $\mathrm{pH}$ range of the samples. Given the high clay content of the sedimentary bedrock, higher concentrations could be related to colloidal aluminum not removed by sample filtration. Relatively high concentrations of barium (range less than 1 to $199 \mu \mathrm{g} / \mathrm{L}$ ) were reported for lakes sampled in the 1980s as part of a baseline waterquality study (Ellis and others, 1992). Many barium concentrations were above the mean concentration of $45 \mu \mathrm{g} / \mathrm{L}$ for large rivers in North America (Hem, 1985) indicating a weathering source in the sedimentary bedrock. This result also may reflect the low sulfate concentration of lakes as barium concentrations in natural waters are controlled partially by the solubility of barium sulfate (Hem, 1985). 

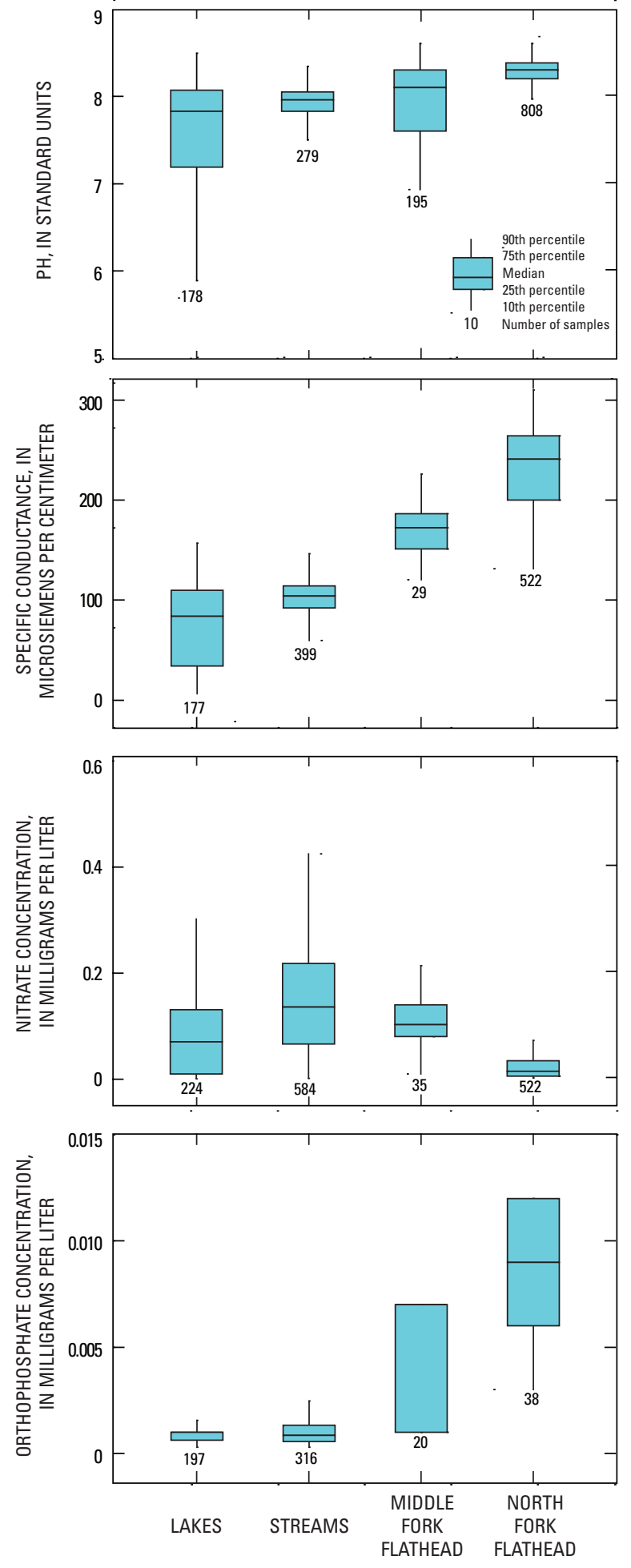

Figure 9. Spatial variation in surface-water quality at selected sites in Glacier National Park, Montana.
In contrast to sites in the park, trace elements in unfiltered samples primarily were measured at sites along the North and Middle Forks of the Flathead River (table 10). This occurrence is because Montana aquatic-life standards are defined for total recoverable rather than dissolved trace elements. The majority of available trace-element data (more than 90 percent) is for the North Fork of the Flathead River at the U.S.-Canada border (N1 and N2). With the exception of aluminum and iron, trace-element concentrations were low, which is not unexpected given the neutral $\mathrm{pH}$ of river water and the lack of mining and urban sources upstream from the site. Concentrations of trace elements were highest in April, May, and June reflecting greater sediment transport by rivers during snowmelt. Aluminum and iron concentrations exceeded $500 \mu \mathrm{g} / \mathrm{L}$ in 10 percent of samples, nearly all of which were collected during high-flow conditions in spring.

Five surface-water sites in GLAC had sufficient data to test for trends in water quality with time, including one site on the Middle Fork (M6), three sites on the North Fork (N1, N2, N6), and one site on Swiftcurrent Creek (S81). All sites were tested for trends in discharge and specific conductance, and two sites on the North Fork also were tested for trends in major constituents and selected nutrients (table 11). None of the sites had significant trends in discharge or specific conductance. Statistically significant trends $(\mathrm{p} \leq 0.01)$ were detected only for sulfate and total phosphorus at Flathead River at the Canada-U.S. border (N2). The magnitude and direction of the sulfate trend were similar for the unadjusted and flow-adjusted concentrations indicating the trend is not related to variations in stream discharge. The cause of the downward trend in sulfate is not clear, particularly considering that a similar trend was not detected in sulfate at site N1. Although the period of record was slightly different, these two sites are located within $100 \mathrm{~m}$ of each other on opposite sides of the CanadaU.S. border. A possible explanation could be that the trend is an artifact of changes in analytical methods. Mast and Turk (1999) observed similar sulfate trends at streams in the USGS Hydrologic Benchmark Network, which were attributed to documented changes in the analytical method for sulfate used by USGS laboratories between 1980 and 1990 (Fishman and others, 1994). A small downward trend in total phosphorus also was detected at site $\mathrm{N} 2$ although the significance of the trend declined slightly after flow adjustment. The magnitude of the trend was small [-0.001 milligrams per liter per year $(\mathrm{mg} / \mathrm{L} / \mathrm{yr})]$ and, as with sulfate, was not detected at site N1. Trends in phosphorus concentrations might reflect changes in land-use activities outside the park boundary that result in sedimentation to the river. Alternatively, the trend might reflect a change in the reporting level for total phosphorus that occurred in 1988.

Comparison to Water-Quality Standards. Water-use classifications and water-quality standards for all stream segments in Montana are established by the MTDEQ (http://www.deq. state.mt.us/wqinfo/Standards/Index.asp, accessed November 2006). The main stems of the North and Middle Forks of the Flathead River above their junction are classified as A-1, 
Table 11. Results of the seasonal Kendall test for trends in streamflow and unadjusted and flow-adjusted constituent concentrations for selected surface-water stations in Glacier National Park, Montana.

$[--$, not calculated or flow model not significant at $\mathrm{p} \leq 0.1 ; \mathrm{ft} / \mathrm{s} / \mathrm{yr}$, cubic feet per second per year; $\mu \mathrm{S} / \mathrm{cm} / \mathrm{yr}$, microsiemens per centimeter per year; $\mathrm{mg} / \mathrm{L} / \mathrm{yr}$, milligrams per liter per year; trends in bold are significant at $\mathrm{p} \leq 0.01$ ]

\begin{tabular}{|c|c|c|c|c|c|}
\hline \multirow[b]{2}{*}{ Constituent or property } & \multicolumn{2}{|c|}{ Unadjusted } & \multicolumn{2}{|c|}{ Flow-adjusted } & \multirow[b]{2}{*}{ Period of record } \\
\hline & Trend & p-value & Trend & p-value & \\
\hline \multicolumn{6}{|c|}{ Middle Fork Flathead River near West Glacier, Montana (M6 in fig. 5) } \\
\hline Streamflow $\left(\mathrm{ft}^{3} / \mathrm{s} / \mathrm{yr}\right)$ & -3.4 & 0.724 & -- & -- & 1982-2004 \\
\hline Specific conductance $(\mu \mathrm{S} / \mathrm{cm} / \mathrm{yr})$ & -.46 & .114 & -0.50 & 0.135 & 1982-2004 \\
\hline \multicolumn{6}{|c|}{ Swiftcurrent Creek at Many Glacier, Montana (S81 in fig. 5) } \\
\hline Discharge $\left(\mathrm{ft}^{3} / \mathrm{s} / \mathrm{yr}\right)$ & .22 & 0.913 & -- & -- & 1982-2004 \\
\hline Specific conductance $(\mu \mathrm{S} / \mathrm{cm} / \mathrm{yr})$ & -.42 & .161 & -0.56 & 0.070 & 1982-2004 \\
\hline \multicolumn{6}{|c|}{ North Fork Flathead River near Columbia Falls, Montana (N6 in fig. 5) } \\
\hline Discharge $\left(\mathrm{ft}^{3} / \mathrm{s} / \mathrm{yr}\right)$ & -2.6 & 0.845 & -- & -- & 1982-2004 \\
\hline Specific conductance $(\mu \mathrm{S} / \mathrm{cm} / \mathrm{yr})$ & .50 & .070 & 0.37 & 0.041 & 1982-2004 \\
\hline \multicolumn{6}{|c|}{ Flathead River at Canada-U.S. Border (N2 in fig. 5) } \\
\hline Discharge $\left(\mathrm{ft}^{3} / \mathrm{s} / \mathrm{yr}\right)$ & 1.29 & 0.592 & -- & -- & 1974-93 \\
\hline $\mathrm{pH}$ (standard units/yr) & .00 & .845 & -- & -- & 1974-93 \\
\hline Specific conductance $(\mu \mathrm{S} / \mathrm{cm} / \mathrm{yr})$ & .00 & .917 & 0.39 & 0.307 & 1974-93 \\
\hline Alkalinity (mg/L/yr) & .00 & .777 & .11 & .632 & 1974-93 \\
\hline Calcium (mg/L/yr) & .00 & .567 & .09 & .030 & 1974-93 \\
\hline Chloride (mg/L/yr) & .00 & .947 & -- & -- & 1974-93 \\
\hline Magnesium (mg/L/yr) & .00 & .896 & .01 & .251 & 1974-93 \\
\hline Potassium (mg/L/yr) & .00 & .650 & .00 & .608 & 1974-93 \\
\hline Sodium (mg/L/yr) & .01 & .014 & -- & -- & 1974-93 \\
\hline Sulfate (mg/L/yr) & -.06 & .013 & -.07 & .005 & 1974-93 \\
\hline Nitrate (mg/L as N/yr) & .001 & .049 & -- & -- & 1974-93 \\
\hline Phosphorus, total (mg/L as P/yr) & -.001 & .010 & -.001 & .014 & 1974-93 \\
\hline \multicolumn{6}{|c|}{ Flathead River at British Columbia (N1 in fig. 5) } \\
\hline Discharge $\left(\mathrm{ft}^{3} / \mathrm{s} / \mathrm{yr}\right)$ & -3.0 & 0.469 & -- & -- & 1979-95 \\
\hline $\mathrm{pH}$ (standard units/yr) & .00 & .683 & -- & -- & 1979-95 \\
\hline Specific conductance $(\mu \mathrm{S} / \mathrm{cm} / \mathrm{yr})$ & 1.08 & .022 & 0.74 & 0.132 & 1979-95 \\
\hline Alkalinity (mg/L/yr) & .50 & .089 & .23 & .014 & 1979-95 \\
\hline Calcium (mg/L/yr) & .15 & .098 & .05 & .352 & 1979-95 \\
\hline Chloride (mg/L/yr) & .00 & .055 & -- & -- & 1979-95 \\
\hline Magnesium (mg/L/yr) & .05 & .101 & .05 & .131 & 1979-95 \\
\hline Potassium (mg/L/yr) & .00 & .016 & -- & -- & 1979-95 \\
\hline Sodium (mg/L/yr) & .00 & .933 & -- & -- & 1979-95 \\
\hline Sulfate (mg/L/yr) & .07 & .133 & .07 & .133 & 1979-95 \\
\hline Nitrate (mg/L as N/yr) & .000 & .544 & -- & -- & 1979-95 \\
\hline Phosphorus, total (mg/L as $\mathrm{P} / \mathrm{yr})$ & .000 & .515 & .000 & .984 & 1980-95 \\
\hline
\end{tabular}


which is surface water suitable for domestic water supply; bathing, swimming, and recreation; growth and propagation of salmonid fishes and associated aquatic life, waterfowl and furbearers; and agricultural and industrial water supply. All surface waters located wholly within the boundaries of GLAC are designated as Outstanding Resource Waters, which offers the highest level of protection in the State. For these water bodies, any new or increased point source discharge that would result in a permanent change in water quality is prohibited. As mentioned previously, Big Creek and Coal Creek, two west-side tributaries of the North Fork outside the park were on the State's 303(d) list in 2006 for sedimentation and siltation related to road networks and timber harvesting. Along the east boundary of the park, Divide Creek was listed as partially impaired in 2006 for cold water fisheries and aquatic life because of road construction and land development around Saint Mary (fig. 5).

Montana aquatic-life and human-health standards are compared to surface-water quality for GLAC in tables 9 and 10. For the more than 900 water-quality samples collected inside the park, only a few samples exceeded the aquatic-life standards for aluminum and lead. For the North and Middle Forks of the Flathead River there were several exceedances of trace-element standards, most notably for aluminum (29 percent) and iron ( 5 percent) in the North Fork. Concentrations of total metals were elevated in these samples because nearly all of them were collected during snowmelt when sediment loads are greater. Because Montana uses total rather than dissolved concentrations for trace-element standards, the standards were exceeded during periods of high sediment transport. There also were a few exceedances for cadmium, copper, lead, manganese, and zinc in samples collected by the USGS during the 1980s. As mentioned previously, sampling methods used by the USGS before 1992 resulted in contamination of dissolved trace elements and data collected before this time have limited qualitative value and should be interpreted with caution.

\section{Ground-Water Quality}

Water-quality data were collected at 22 ground-water wells within the boundaries of GLAC (table 12). For these 22 wells, there are 74 samples with 2,480 individual data results including field properties (14 percent), and major-constituent (45 percent), nutrient (4 percent), and trace-element (29 percent) analyses. Five of the wells (G10, G11, G12, G21, G22) were sampled once each by the USGS in 1972 as part of a study to evaluate ground-water supplies at campgrounds and ranger stations in the park (Boettcher, 1973). The remaining 17 wells were sampled four times each during 1980-81. These are shallow observation wells completed in alluvium and located in the vicinity of park wastewater facilities in West Glacier, Saint Mary, and Many Glacier (table 12). They were sampled to determine if effluent from the wastewater treatment facilities was affecting the water quality of the alluvial aquifer (Moreland and Wood, 1982). No ground-water-quality data are available for the park for the past 25 years.
Ground-water samples had low to moderate concentrations of dissolved solids and were well buffered (table 13). Specific conductance ranged from 59 to $445 \mu \mathrm{S} / \mathrm{cm}$, and alkalinity ranged from 33 to $290 \mathrm{mg} / \mathrm{L}$. The major cation in ground water was calcium and the dominant anion was bicarbonate (as indicated by $\mathrm{pH}$ and alkalinity). The predominance of these ions is due to weathering of carbonate minerals in sedimentary rocks that occur as fragments in alluvium and till. Chloride was low (median $0.7 \mathrm{mg} / \mathrm{L}$ ), characteristic of an atmospheric source (Hem, 1985), whereas sulfate was slightly higher (median $5.8 \mathrm{mg} / \mathrm{L}$ ) indicating an additional source from weathering. Nutrient concentrations were low in all the well samples; nitrate ranged from 0.01 to $1.1 \mathrm{mg} / \mathrm{L}$, orthophosphate from 0.02 to $0.04 \mathrm{mg} / \mathrm{L}$ and total phosphorus from 0.003 to $0.13 \mathrm{mg} / \mathrm{L}$. Nutrients and major constituents in wells sampled during 1980 and 1981 did not appear to be affected by nearby wastewater treatment facilities (Moreland and Wood, 1982). However, many of the wells were upgradient from the facilities, or were completed in aquifers that were not hydrologically connected to the percolation ponds (Moreland and Wood, 1982). Of the trace elements, iron and manganese were present in the largest concentrations. This likely is because of reductive dissolution of aquifer sediments, although this cannot be verified because dissolved oxygen was not measured. A few samples had detectable concentrations of other trace elements most notably for copper, lead, and zinc. These detections were somewhat unexpected given the neutral $\mathrm{pH}$ measured in these well water samples and the fact that alluvial material is composed of unmineralized sedimentary rock types. A possible explanation for the detected trace-element concentrations is contamination of samples during collection and processing. It has been documented that samples collected for dissolved trace elements by the USGS before 1992 were sometimes contaminated during sampling and field processing (http://water. usgs.gov/admin/memo/QW/qw91.10.html, accessed November 2006). The contamination was most pronounced for arsenic, boron, beryllium, cadmium, chromium, copper, lead, and zinc. Because of the contamination issue, trace-element data for ground-water wells in GLAC must be viewed as questionable, and should be interpreted with caution. None of the wells had sufficient data to determine trends in ground-water quality or water levels with time.

Comparison to Water-Quality Standards. Although the sampled wells in the park are not used for water supplies, samples were compared to water-supply standards that are established by the MTDEQ for ground water (http://www.deq.state. mt.us/wqinfo/Standards/Index.asp, accessed November 2006). Primary drinking-water standards are developed for human health, and secondary standards are based on aesthetic properties such as taste, odor, and staining. Several of the samples collected during 1980 and 1981 had constituent concentrations that exceeded the human-health standards for cadmium and lead (table 13). One sample exceeded the secondary standard for manganese, and three exceeded the secondary standard for iron. As mentioned previously, sampling methods used by the USGS before 1993 could have resulted in contamination of 
Table 12. Ground-water sampling sites in Glacier National Park, Montana.

[no., number; well depth in meters; WTF, wastewater treatment facility CG, campground; RS, ranger station; identification numbers and station names from USGS NWIS]

\begin{tabular}{|c|c|c|c|c|c|c|c|}
\hline $\begin{array}{c}\text { Site no. } \\
\text { (fig. 5) }\end{array}$ & Identification no. & Station name & Latitude & Longitude & $\begin{array}{l}\text { Well } \\
\text { depth }\end{array}$ & Location & $\begin{array}{l}\text { Period of } \\
\text { record }\end{array}$ \\
\hline G1 & 483029113595901 & 32N19W26BCCC01 & $48^{\circ} 30^{\prime} 28^{\prime \prime}$ & $114^{\circ} 00^{\prime} 02^{\prime \prime}$ & 4.0 & West Glacier WTF & $1980-81$ \\
\hline G2 & 483031114001601 & 32N19W27ADCD01 & $48^{\circ} 30^{\prime} 02^{\prime \prime}$ & $114^{\circ} 00^{\prime} 19^{\prime \prime}$ & 8.7 & West Glacier WTF & $1980-81$ \\
\hline G3 & 483036114000401 & 32N19W27ADDA01 & $48^{\circ} 30^{\prime} 35^{\prime \prime}$ & $114^{\circ} 00^{\prime} 07^{\prime \prime}$ & 3.1 & West Glacier WTF & $1980-81$ \\
\hline G4 & 483037114000501 & 32N19W27ADAD03 & $48^{\circ} 30^{\prime} 36^{\prime \prime}$ & $114^{\circ} 00^{\prime} 08^{\prime \prime}$ & 3.2 & West Glacier WTF & $1980-81$ \\
\hline G5 & 483038114000601 & 32N19W27ADAD02 & $48^{\circ} 30^{\prime} 37^{\prime \prime}$ & $114^{\circ} 00^{\prime} 09^{\prime \prime}$ & 3.2 & West Glacier WTF & $1980-81$ \\
\hline G6 & 483039114000701 & 32N19W27ADAD01 & $48^{\circ} 30^{\prime} 38^{\prime \prime}$ & $114^{\circ} 00^{\prime} 10^{\prime \prime}$ & 2.3 & West Glacier WTF & $1980-81$ \\
\hline G7 & 483039114000801 & 32N19W27ADAA01 & $48^{\circ} 30^{\prime} 38^{\prime \prime}$ & $114^{\circ} 00^{\prime} 11^{\prime \prime}$ & 3.5 & West Glacier WTF & $1980-81$ \\
\hline G8 & 483039114001301 & 32N19W27ADAB01 & $48^{\circ} 30^{\prime} 38^{\prime \prime}$ & $114^{\circ} 00^{\prime} 16^{\prime \prime}$ & 10.0 & West Glacier WTF & $1980-81$ \\
\hline G9 & 483045113595401 & 32N19W26BBCD01 & $48^{\circ} 30^{\prime} 44^{\prime \prime}$ & $113^{\circ} 59^{\prime} 57^{\prime \prime}$ & 12.0 & West Glacier WTF & $1980-81$ \\
\hline G10 & 484153114113300 & 34N20W20DDA 01 & $48^{\circ} 41^{\prime} 52^{\prime \prime}$ & $114^{\circ} 11^{\prime} 36^{\prime \prime}$ & 12.5 & Logging Creek CG & 1972 \\
\hline G11 & 484155114112600 & 34N20W21BCB 01 & $48^{\circ} 41^{\prime} 54^{\prime \prime}$ & $114^{\circ} 11^{\prime} 29^{\prime \prime}$ & 10.4 & Logging Creek CG & 1972 \\
\hline G12 & 484321114132500 & 34N20W07DBB 01 & $48^{\circ} 43^{\prime} 20^{\prime \prime}$ & $114^{\circ} 13^{\prime} 28^{\prime \prime}$ & 13.7 & Quartz Creek CG & 1972 \\
\hline G13 & 484427113254801 & 35N14W34CCCA01 & $48^{\circ} 44^{\prime} 26^{\prime \prime}$ & $113^{\circ} 25^{\prime} 51^{\prime \prime}$ & 10.3 & Saint Mary WTF & $1980-81$ \\
\hline G14 & 484434113260701 & 35N14W33DDBA01 & $48^{\circ} 44^{\prime} 33^{\prime \prime}$ & $113^{\circ} 26^{\prime} 10^{\prime \prime}$ & 22.9 & Saint Mary WTF & $1980-81$ \\
\hline G15 & 484437113255601 & 35N14W33DADD01 & $48^{\circ} 44^{\prime} 36^{\prime \prime}$ & $113^{\circ} 25^{\prime} 59^{\prime \prime}$ & 24.4 & Saint Mary WTF & $1980-81$ \\
\hline G16 & 484439113260101 & 35N14W33DADC01 & $48^{\circ} 44^{\prime} 38^{\prime \prime}$ & $113^{\circ} 26^{\prime} 04^{\prime \prime}$ & 23.9 & Saint Mary WTF & $1980-81$ \\
\hline G17 & 484803113384301 & 35N16W12DCCA01 & $48^{\circ} 48^{\prime} 02^{\prime \prime}$ & $113^{\circ} 38^{\prime} 46^{\prime \prime}$ & 10.9 & Many Glacier WTF & $1980-81$ \\
\hline G18 & 484804113383901 & 35N16W12CBBB01 & $48^{\circ} 48^{\prime} 03^{\prime \prime}$ & $113^{\circ} 38^{\prime} 42^{\prime \prime}$ & 11.6 & Many Glacier WTF & $1980-81$ \\
\hline G19 & 484806113384501 & 35N16W12CCAB01 & $48^{\circ} 48^{\prime} 05^{\prime \prime}$ & $113^{\circ} 38^{\prime} 48^{\prime \prime}$ & 9.1 & Many Glacier WTF & $1980-81$ \\
\hline G20 & 484808113384101 & 35N16W12CACC01 & $48^{\circ} 48^{\prime} 07^{\prime \prime}$ & $113^{\circ} 38^{\prime} 44^{\prime \prime}$ & 10.6 & Many Glacier WTF & $1980-81$ \\
\hline $\mathrm{G} 21$ & 484817114190900 & 35N21W09CCA 01 & $48^{\circ} 48^{\prime} 16^{\prime \prime}$ & $114^{\circ} 19^{\prime} 12^{\prime \prime}$ & 6.1 & Polebridge RS & 1972 \\
\hline G22 & 485609114204400 & 37N21W29DDC 01 & $48^{\circ} 56^{\prime} 08^{\prime \prime}$ & $114^{\circ} 20^{\prime} 47^{\prime \prime}$ & 14.0 & Kintla Lake CG & 1972 \\
\hline
\end{tabular}

dissolved trace elements, so data collected before this time have limited qualitative value and should be interpreted with caution.

\section{Summary and Suggestions for Vital Signs Monitoring}

The historical water-quality data review identified 1,144 samples collected at 54 streams and 36 lakes in GLAC from 1963 to 2004. The majority of results for these samples were field properties (19 percent), and major-constituent (30 percent), nutrient (26 percent), trace-element ( 8 percent), and organic carbon ( 7 percent) analyses. Most of the surface-water sites were sampled 1 to 3 times each during the period of record, although there were 14 sites that were sampled 20 or more times. For the 15 main-stem sites along the North and Middle Forks of the Flathead River, there were 1,515 samples collected from 1949 to 2004. Streamflow-gaging stations currently (2007) are operated by the USGS at six stations in the park including Swiftcurrent Creek at Many Glacier, which has been operated since 1912 and is the longest continuously operating station in the park. Surface water is a moderately dilute calcium-bicarbonate type, and dissolved solids primarily are derived from weathering of carbonate minerals in the sedimentary bedrock. Wildfire was determined to be an important control on stream-water nutrients in areas of the park burned during 2003. Five sites had sufficient data to test for temporal trends in water quality. The only detected trends were attributed to analytical method-related factors, rather than environmental change. All streams and lakes located entirely within the boundaries of GLAC are designated as Outstanding Resource Waters, which offers the highest level of protection in Montana. Historical data indicates water quality in the park meets Montana aquatic-life and human-health standards. Ground-water quality and resources in the park are not well characterized, and no water-quality data are available for the park for the past 25 years.

Some water-quality issues in GLAC include effects of visitor use and park facilities, atmospheric deposition of pollutants to high-elevation lakes, and land-use activities in areas 
Table 13. Summary of selected water-quality data for ground-water sites in Glacier National Park, Montana, 1972 to 1981.

[no., number; human-health standards from www.deq.state.mt.us/wqinfo/Standards/Index.asp; <, less than; --, not reported; ${ }^{\circ} \mathrm{C}$, degrees Celsius; $\mu \mathrm{S} / \mathrm{cm}$, microsiemens per centimeter at $25^{\circ} \mathrm{C} ; \mathrm{mg} / \mathrm{L}$, milligrams per liter; $\mathrm{CaCO}_{3}$, calcium carbonate; $\mathrm{N}$, nitrogen; $\mathrm{P}$, phosphorous; $\mu \mathrm{g} / \mathrm{L}$, micrograms per liter; some trace elements have multiple reporting limits]

\begin{tabular}{|c|c|c|c|c|c|c|}
\hline Constituent or property & $\begin{array}{l}\text { No. } \\
\text { sites }\end{array}$ & $\begin{array}{l}\text { No. analyses } \\
\text { (no. censored) }\end{array}$ & $\begin{array}{l}\text { Minimum } \\
\text { value }\end{array}$ & $\begin{array}{c}\text { Median } \\
\text { value }\end{array}$ & $\begin{array}{l}\text { Maximum } \\
\text { value }\end{array}$ & $\begin{array}{l}\text { Human-health } \\
\text { standard }\end{array}$ \\
\hline \multicolumn{7}{|c|}{ Field properties } \\
\hline Temperature, water $\left({ }^{\circ} \mathrm{C}\right)$ & 22 & 72 & 3.0 & 7.5 & 15 & -- \\
\hline pH (standard units) & 22 & 67 & 6.90 & 7.50 & 8.20 & -- \\
\hline Specific conductance $(\mu \mathrm{S} / \mathrm{cm})$ & 22 & 72 & 59 & 301 & 445 & -- \\
\hline \multicolumn{7}{|c|}{ Major constituents } \\
\hline Alkalinity $\left(\mathrm{mg} / \mathrm{L}\right.$ as $\left.\mathrm{CaCO}_{3}\right)$ & 22 & 74 & 33 & 185 & 290 & -- \\
\hline Calcium, dissolved (mg/L) & 22 & 74 & 7.4 & 43 & 69 & -- \\
\hline Chloride, dissolved (mg/L) & 22 & 73 & .1 & .7 & 3.0 & -- \\
\hline Fluoride, dissolved (mg/L) & 22 & $74(1)$ & a .01 & .06 & .2 & 4.0 \\
\hline Magnesium, dissolved (mg/L) & 22 & 74 & 2.1 & 11 & 29 & -- \\
\hline Potassium, dissolved (mg/L) & 22 & 74 & .1 & .5 & 3.0 & -- \\
\hline Silica, dissolved (mg/L) & 22 & 74 & 3.8 & 5.8 & 28 & -- \\
\hline Sodium, dissolved (mg/L) & 22 & 74 & .5 & 1.3 & 5.2 & -- \\
\hline Sulfate, dissolved $(\mathrm{mg} / \mathrm{L})$ & 22 & 74 & 6 & 5.8 & 39 & -- \\
\hline \multicolumn{7}{|c|}{ Nutrients } \\
\hline Nitrate, dissolved (mg/L as $\mathrm{N})$ & 22 & 74 & 0.01 & 0.15 & 1.1 & 10 \\
\hline Orthophosphate, dissolved (mg/L as P) & 5 & 5 & .02 & .02 & .04 & -- \\
\hline Phosphorus, total (mg/L as P) & 13 & $26(1)$ & ${ }^{\mathrm{a}} .003$ & .018 & .13 & -- \\
\hline \multicolumn{7}{|c|}{ Trace elements } \\
\hline Aluminum, dissolved ( $\mu \mathrm{g} / \mathrm{L})$ & 17 & 39 (14) & $<20$ & 50 & 150 & -- \\
\hline Cadmium, dissolved ( $\mu \mathrm{g} / \mathrm{L})$ & 17 & $39(28)$ & $<2$ & $<2$ & 9 & 5 \\
\hline Chromium, dissolved $(\mu \mathrm{g} / \mathrm{L})$ & 17 & $39(20)$ & $<2$ & $<2$ & 20 & 100 \\
\hline Copper, dissolved $(\mu \mathrm{g} / \mathrm{L})$ & 17 & $39(7)$ & $<2$ & 5 & 12 & 1,300 \\
\hline Iron, dissolved $(\mu \mathrm{g} / \mathrm{L})$ & 22 & $74(26)$ & $<2$ & 20 & 2,300 & b300 \\
\hline Lead, dissolved ( $\mu \mathrm{g} / \mathrm{L})$ & 17 & $39(31)$ & $<10$ & $<40$ & 290 & 15 \\
\hline Lithium, dissolved ( $\mu \mathrm{g} / \mathrm{L})$ & 22 & $69(49)$ & $<2$ & $<2$ & 10 & -- \\
\hline Manganese, dissolved $(\mu \mathrm{g} / \mathrm{L})$ & 22 & $74(25)$ & $<0$ & $<2$ & 240 & b50 \\
\hline Molybdenum, dissolved ( $\mu \mathrm{g} / \mathrm{L})$ & 17 & $39(22)$ & $<10$ & 20 & 38 & -- \\
\hline Nickel, dissolved ( $\mu \mathrm{g} / \mathrm{L})$ & 17 & $39(31)$ & $<10$ & $<10$ & $<60$ & 100 \\
\hline Silver, dissolved ( $\mu \mathrm{g} / \mathrm{L})$ & 17 & $39(27)$ & $<2$ & $<2$ & 9 & 100 \\
\hline Titanium, dissolved $(\mu \mathrm{g} / \mathrm{L})$ & 17 & $39(10)$ & $<1$ & 4 & 20 & -- \\
\hline Vanadium, dissolved ( $\mu \mathrm{g} / \mathrm{L})$ & 17 & $39(24)$ & $<1$ & $<1$ & 28 & -- \\
\hline Zinc, dissolved ( $\mu \mathrm{g} / \mathrm{L})$ & 17 & $39(22)$ & $<3$ & $<3$ & 540 & 2,000 \\
\hline Zirconium, dissolved ( $\mu \mathrm{g} / \mathrm{L})$ & 17 & $39(34)$ & $<4$ & $<4$ & $<50$ & -- \\
\hline
\end{tabular}

${ }^{a}$ Minimum reported value less than minimum censored value.

${ }^{\mathrm{b}}$ Secondary standard based on aesthetic properties such as taste, odor, and staining. 
outside the park draining the North and Middle Forks of the Flathead River. Climate change may have long-term effects on water resources in the park. Based on historical data review, the following suggestions are provided for consideration in designing a water-quality monitoring plan for GLAC.

- Maintain long-term water-quality monitoring at Swiftcurrent Creek above Swiftcurrent Lake (S78) and establish as a sentinel site for the east side of the park. The site is currently sampled as frequently as 20 times per year for major constituents and nutrients as part of the USGS Hydrologic Benchmark Network and a USGS streamflow-gaging station has been operated here since 2001 .

- Consider adding a sentinel site on the west side of the park such as Avalanche Creek (S6) or McDonald Creek above Lake McDonald (S47). Both sites were sampled for nutrients and were gaged for streamflow from 1993 through 1998 by the NPS.

- Conduct periodic surveys of high-elevation lakes in the park to monitor the effects of atmospheric deposition of contaminants. Lakes could be selected on the basis of historical water-quality data and statistical models that estimate lake sensitivity to nitrogen and sulfur deposition (Nanus and others, 2005).

- Establish a network of streamflow-gaging stations and ground-water wells in the park to monitor the effect of climate change on water resources.

\section{Grant-Kohrs Ranch National Historic Site}

\section{Environmental Setting and Land Use}

Grant-Kohrs Ranch National Historic Site (GRKO) is located at the north end of the Deer Lodge Valley (fig. 10), which is an intermontane basin in western Montana bounded by low mountains to the east and the rugged Flint Creek Range to the west. The park covers an area of $6.5 \mathrm{~km}^{2}$ and is situated in the floodplain of the Clark Fork River, which bisects the park from south to north. Only a small area in the southwest corner of the park extends onto terraces above the floodplain. Vegetation in riparian areas is dominated by redtop and willows, and drier areas of the park are used for pasture and for cultivation of hay (Britten and others, 2006). The climate is semiarid and typical of colder short-grass prairies. Mean monthly air temperatures at Deer Lodge range from $-6.3^{\circ} \mathrm{C}$ in January to $16.4^{\circ} \mathrm{C}$ in July (http://www.wrcc.dri. edu/cgi-bin/cliMAIN.pl?mtdeer, accessed June 2006). Annual average precipitation is $27 \mathrm{~cm}$, most of which occurs as rain in late spring and early summer. The area receives about 92 $\mathrm{cm}$ of snow each year between October and April. Bedrock underlying the northern part of the Deer Lodge Valley includes a thick sequence of Tertiary sediments composed of grayish to pale orange sandy or silty mudstones interspersed with sand, gravel, and pebble conglomerate beds (Berg, 2004). In GRKO, the Tertiary sediments are covered by Quaternary alluvium and colluvium, and alluvial terrace deposits composed of poorly sorted gravel, sand, silt, and clay derived from Tertiary and older strata in the surrounding highlands (Berg, 2004).

GRKO is managed as a working cattle ranch and the site preserves 90 historic structures associated with its operation (http://www.nps.gov/grko/parkmgmt/planning.htm, accessed June 2006). Land-use activities primarily are related to cattle grazing, hay cultivation, and cultural feature operation and maintenance. The park has as many as 25 water-right claims to surface water from the river and its tributaries for flood irrigation of park lands. GRKO obtains drinking water from the town of Deer Lodge municipal system, and wastewater is handled by the Deer Lodge wastewater treatment facility, located just inside the northern park boundary (fig. 10). Since 2000, the ranch has used secondary treated sewage effluent from the wastewater treatment facility to irrigate some of the hayfields during summer months. For example, in June, July, and August of 2000 and 2001, $0.48 \mathrm{~km}^{2}$ of park land was irrigated with 310 and 650 million liters of effluent, respectively (Johnson, 2002). This arrangement is part of the Clark Fork River Voluntary Nutrient Reduction Program (VNRP), which was developed by stakeholders to reduce nutrient pollution in the upper and middle reaches of the Clark Fork River (http:// www.tristatecouncil.org/programs/cfrwatershed.html, accessed June 2006). The Clark Fork River has some of the most productive stream water in western Montana, and some of the highest densities of attached algae are present in the reach just downstream from GRKO. In 2000, the NPS studied the fate of sewage effluent nutrients (primarily nitrogen) applied to soils in GRKO by a sprinkler irrigation system (Johnson, 2002). The study determined that most of the applied nitrogen was retained by plants and in the soil, but that as much as 40 percent of the nitrogen might be leached to the shallow ground-water system.

GRKO also is within the Clark Fork River Superfund Site (http://www.epa.gov/region08/superfund/sites/mt/milltowncfr/ cfrou.html, accessed June 2006), which is contaminated with arsenic and heavy metals (copper, cadmium, lead, and zinc) related to historic mining, milling, and smelting activities in the headwaters of the Clark Fork River. Tailings from these operations were transported downstream and deposited along the floodplain of the Clark Fork River by at least six major floods in the late 1800s and early 1900s (Smith and others, 1998). Sedimentation ponds were later built to prevent movement of tailings from headwater areas; however, the river continues to be contaminated by erosion of previously deposited tailings, and by direct leaching of metals from tailings into surface and ground water. Copper is the primary metal of concern because it is the most toxic to aquatic life and plants in the river floodplain (Lambing, 1991). An NPS study in 2000 estimated the volume of contaminated geologic resources in GRKO (Moore and Woessner, 2000) to include $12,600 \mathrm{~m}^{2}$ of exposed tailings and as much as 1,660,000 cubic meters $\left(\mathrm{m}^{3}\right)$ of contaminated soil. In April, 2004, a Record of Decision was issued by the USEPA for the remediation of contaminated areas within Reach A of the Clark Fork River, which includes GRKO 


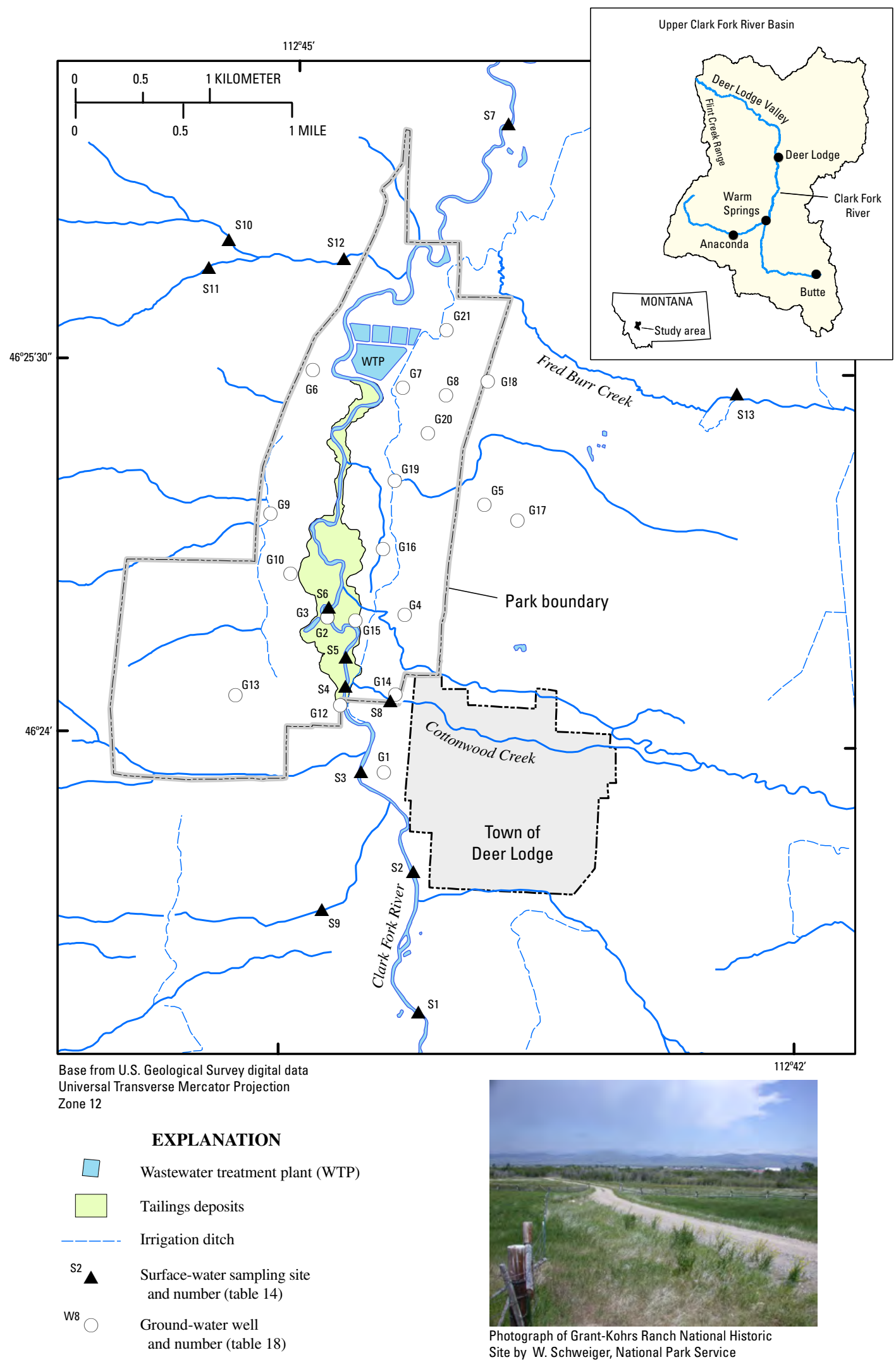

Figure 10. Location of water-quality sampling sites near Grant-Kohrs Ranch National Historic Site, Montana. 
(http://www.epa.gov/region8/superfund/sites/mt/milltowncfr/ cfrrodhome.html, accessed June 2006). The remediation will include removal of larger tailing deposits $\left(>37 \mathrm{~m}^{2}\right.$ in area and $60 \mathrm{~cm}$ deep) followed by revegetation of the excavated area. Smaller tailing deposits (generally what are present in GRKO) and impacted soil and vegetation will be remediated in place using lime addition, soil mixing, and revegetation.

\section{Water Resources}

GRKO is situated along a 5-km reach of the Clark Fork River downstream from the town of Deer Lodge, between Cottonwood Creek and Fred Burr Creek. The Clark Fork River begins near the town of Warm Springs and flows northward through the broad and meandering floodplain of the Deer Lodge Valley with an average gradient of 3.8 meters per kilometer $(\mathrm{m} / \mathrm{km})$. The river has four west-side perennial tributaries that flow from the Flint Creek Range, and several small east-side tributaries that flow only during spring snowmelt. During the summer months, water from the Clark Fork River and its west-side tributary streams is diverted for irrigation (Smith and others, 1998), some of which returns to the river channel as surface- or ground-water return flow.

The USGS has operated as many as 22 streamflowgaging stations (10 were active in 2006) in the upper Clark Fork River Basin (http://mt.water.usgs.gov/pub/MTStations. $p d f$, accessed June 2006), including the Clark Fork River at Deer Lodge (USGS streamflow-gaging station 12324200) just $500 \mathrm{~m}$ upstream from GRKO, which has been in operation since 1979 (site S3, fig. 10). In addition to discharge, station 12324200 also has continuous water temperature measurements for 2001 to 2006 and suspended-sediment concentrations for 1985 to 2006 (http://waterdata.usgs.gov/ nwis/inventory/?site_no $=12324200$, accessed June 2006). The range of daily streamflow values for the Clark Fork River at Deer Lodge from 1979 to 2005 is shown in figure 11. Peak flows typically occur in late May and early June as a result of spring rains and melting of mountain snowpacks. Runoff during snowmelt (April, May, and June) represents 37 percent of the mean annual runoff of $7.5 \mathrm{~cm}$ for the Clark Fork River at Deer Lodge. The lowest flows are in late July and August due to declining runoff and increasing rates of evapotranspiration combined with diversion of water for irrigation. Increased flows also can occur in late winter and early spring because of valley snowmelt and breakup of ice cover on the river, and in summer during thunderstorms (Hornberger and others, 1997).

Ground-water resources in the upper Clark Fork River Basin are described by Nimick (1993) and Konizeski and others (1962). Shallow aquifers in Quaternary and Tertiary valleyfill deposits are the primary water source for domestic supplies and irrigation. The direction of ground-water flow generally is from upland areas towards the Clark Fork River. Recharge to these shallow aquifers is by infiltration of precipitation, losses from irrigation ditches and irrigated lands, and infiltration from streams during periods of increased streamflow. The alluvial aquifer is localized along the river and major tributar-

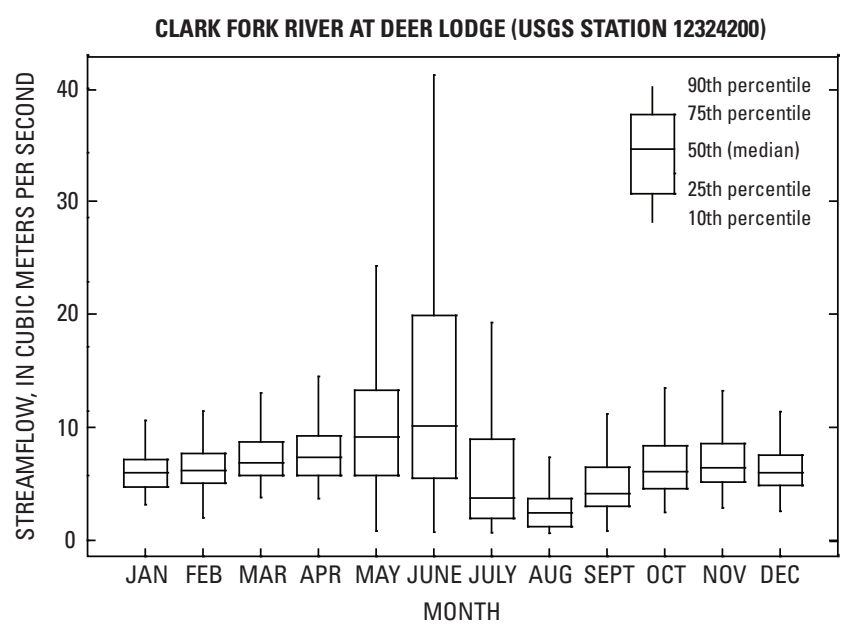

Figure 11. Daily streamflow range at Clark Fork River at Deer Lodge (S3) near Grant-Kohrs Ranch National Historic Site, Montana, 1979-2005.

ies and is composed of medium to well-sorted unconsolidated gravel. Wells in alluvial deposits have a median depth of 11 $\mathrm{m}$, and rarely are deeper than $21 \mathrm{~m}$. Wells yield varies considerably ranging from 0.19 to $37 \mathrm{~L} / \mathrm{s}$ with a median of 2.5 $\mathrm{L} / \mathrm{s}$. Coarse-grained beds and lenses of sand and gravel are the primary units that yield water to wells in the Tertiary aquifer. Wells completed in the Tertiary aquifer generally were deeper (median $33 \mathrm{~m}$ ) and had lower yields (median $1.3 \mathrm{~L} / \mathrm{s}$ ) than wells in the alluvial aquifer. Water-level data are available for several shallow alluvial wells in GRKO. Nimick (1993) reported intermittent water-level measurements for two observation wells (G2 and G3, fig. 10) within GRKO during 1986 and 1987 and at several additional wells just outside the east park boundary. Monthly water-level data were collected during 2000 at 16 monitoring wells (G6-G21, fig. 10) installed as part of a NPS study (Moore and Woessner, 2000). The hydrograph in figure 12 shows water levels at selected wells during 2000. Water levels at many of the wells were higher in late spring and early summer, likely reflecting increased precipitation rates and streamflow during this time of year.

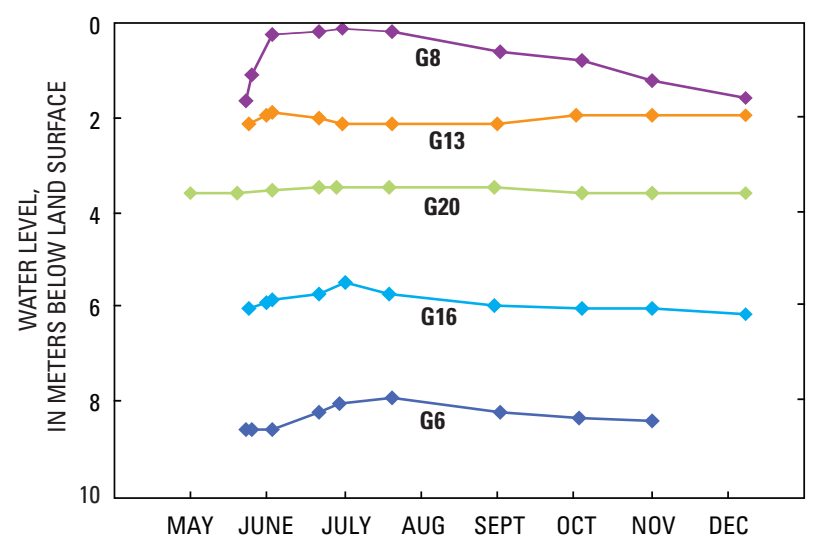

Figure 12. Ground-water level measurements at selected monitoring wells in Grant-Kohrs Ranch National Historic Site, Montana, 2000. 


\section{Surface-Water Quality}

Water-quality data were collected at 13 surface-water sites in and immediately adjacent to GRKO, including 7 sites along the main stem of the Clark Fork River and 6 tributary streams (table 14, fig. 10). Water-quality samples collected at site S3 (Clark Fork River at Deer Lodge) are part of a longterm study being conducted by the USGS to quantify the concentrations, transport, and accumulation of metals in the water, bed sediment, and aquatic insects in the upper Clark Fork River Basin (S9 also sampled as part of this program). These data are being used to identify elevated metal concentrations that could pose an environmental risk, determine source areas of metals, and detect trends with time as remediation of the Superfund site proceeds (Dodge and others, 2005). Water-quality samples were collected intermittently at sites S1, S2, S4, S5, S7, and S8 between 1973 and 1996 as part of a surface-water monitoring program operated by the MTDEQ (http://www.deq.state.mt.us/wqinfo/monitoring/index.asp, accessed June 2006). Diurnal data sets were collected at site S6 (2 km downstream from S3) during July 21-23, 1994, and February 15-17, 1995, by the University of Montana to study diel cycles in arsenic, cadmium, nickel, manganese, and zinc concentrations (Brick and Moore, 1996). Four tributary sites (S10 to S13) were sampled once each during 1976 and 1977 as part of the NURE program (http://pubs.usgs.gov/of/1997/ ofr-97-0492/, accessed June 2006). The ROMN database includes 881 samples collected at these 13 surface-water sites from 1968 to 2004 . The majority of results for these 881 samples were field properties ( 24 percent), and major-constit- uent (24 percent), nutrient ( 9 percent), and trace-element (30 percent) analyses.

Because only a few samples were collected at tributary streams, the following discussion focuses on the water quality of the Clark Fork River. The period of record and types of analyses conducted at the main-stem sites are presented in table 15; water-quality data for 1980 to 2004 are summarized in table 16 . The Clark Fork River samples showed a wide range in composition with specific conductance ranging from 208 to $1,890 \mu \mathrm{S} /$ $\mathrm{cm}$ and alkalinity ranging from 7 to $199 \mathrm{mg} / \mathrm{L}$. Calcium was the dominant cation (median $72 \mathrm{mg} / \mathrm{L}$ ) and bicarbonate (based on $\mathrm{pH}$ and alkalinity; median $142 \mathrm{mg} / \mathrm{L}$ ) and sulfate (median $120 \mathrm{mg} / \mathrm{L}$ ) were the dominant anions. Calcium and bicarbonate (based on $\mathrm{pH}$ and alkalinity) are most likely derived from weathering of carbonate rocks that are common in the basin and as fragments in the valley fill and alluvium. By contrast, most sulfate probably is released from oxidation of sulfide minerals in mine wastes located in waste piles and tailings ponds in the headwater valleys of the Clark Fork and in flood-plain deposits along the river. Concentrations of major dissolved constituents in the river varied seasonally as shown by specific conductance values at site S3 (fig. 13). Concentrations are lowest during increased flow in May and June, when there are large contributions of dilute snowmelt to the river. Concentrations vary little from September through April when streamflow is sustained primarily by ground-water discharge.

Nutrient data for the Clark Fork included ammonia, nitrate, and orthophosphate concentrations, most of which were collected at site $\mathrm{S} 1$, which is located upstream from the town of Deer Lodge, and at site S6, which is located inside

Table 14. Surface-water sampling sites near Grant-Kohrs Ranch Ranch National Historic Site, Montana.

[no., number; MS, main-stem site on Clark Fork River, TR, tributary stream to Clark Fork River; Mont., Montana; NHS, National Historic Site]

\begin{tabular}{|c|c|c|c|c|c|c|}
\hline $\begin{array}{l}\text { Site no. } \\
\text { (fig. 10) }\end{array}$ & $\begin{array}{c}\text { Identification } \\
\text { number }\end{array}$ & Station name & Latitude & Longitude & Type & Period of record \\
\hline $\mathrm{S} 1$ & 3526CL01 & Clark Fork River ${ }^{\mathrm{a}}$ & $46^{\circ} 22^{\prime} 54^{\prime \prime}$ & $112^{\circ} 44^{\prime} 07^{\prime \prime}$ & MS & $1973-96$ \\
\hline S2 & 3526CL10 & Clark Fork River ${ }^{\mathrm{a}}$ & $46^{\circ} 23^{\prime} 30^{\prime \prime}$ & $112^{\circ} 44^{\prime} 02^{\prime \prime}$ & MS & 1978 \\
\hline S3 & 12324200 & Clark Fork at Deer Lodge, Mont. ${ }^{\mathrm{b}}$ & $46^{\circ} 23^{\prime} 51^{\prime \prime}$ & $112^{\circ} 44^{\prime} 34^{\prime \prime}$ & MS & $1968-71,79,82-04$ \\
\hline S4 & 3626CL01 & Clark Fork River ${ }^{\mathrm{a}}$ & $46^{\circ} 24^{\prime} 09^{\prime \prime}$ & $112^{\circ} 44^{\prime} 20^{\prime \prime}$ & MS & $1977-80,85,88,91$ \\
\hline S5 & 3626CL02 & Clark Fork River ${ }^{a}$ & $46^{\circ} 24^{\prime} 18^{\prime \prime}$ & $112^{\circ} 44^{\prime} 25^{\prime \prime}$ & MS & $1977-78,86-87$ \\
\hline S6 & GRKO_BRICK_1 & Clark Fork River at Grant-Kohrs NHS ${ }^{\mathrm{a}}$ & $46^{\circ} 24^{\prime} 31^{\prime \prime}$ & $112^{\circ} 44^{\prime} 45^{\prime \prime}$ & MS & 1994-95 \\
\hline S7 & 3626CL03 & Clark Fork River ${ }^{\mathrm{a}}$ & $46^{\circ} 26^{\prime} 25^{\prime \prime}$ & $112^{\circ} 43^{\prime} 35^{\prime \prime}$ & MS & $1977-80$ \\
\hline S8 & $3626 \mathrm{CO} 01$ & Cottonwood Creek ${ }^{\mathrm{a}}$ & $46^{\circ} 24^{\prime} 09^{\prime \prime}$ & $112^{\circ} 44^{\prime} 21^{\prime \prime}$ & $\mathrm{TR}$ & $1978,88-89$ \\
\hline S9 & 462320112443701 & Tin Cup Joe Creek at Deer Lodge, Mont. & $46^{\circ} 23^{\prime} 19^{\prime \prime}$ & $112^{\circ} 44^{\prime} 40^{\prime \prime}$ & $\mathrm{TR}$ & 1996 \\
\hline S10 & GRKO_NURE_041 & M25833 & $46^{\circ} 25^{\prime} 59^{\prime \prime}$ & $112^{\circ} 45^{\prime} 23^{\prime \prime}$ & $\mathrm{TR}$ & 1977 \\
\hline S11 & GRKO_NURE_042 & M25834 & $46^{\circ} 25^{\prime} 52^{\prime \prime}$ & $112^{\circ} 45^{\prime} 30^{\prime \prime}$ & TR & 1977 \\
\hline $\mathrm{S} 12$ & GRKO_NURE_043 & M24922 & $46^{\circ} 25^{\prime} 55^{\prime \prime}$ & $112^{\circ} 44^{\prime} 43^{\prime \prime}$ & $\mathrm{TR}$ & 1977 \\
\hline S13 & GRKO_NURE_080 & M24438 & $46^{\circ} 25^{\prime} 19^{\prime \prime}$ & $112^{\circ} 42^{\prime} 28^{\prime \prime}$ & TR & 1976 \\
\hline
\end{tabular}

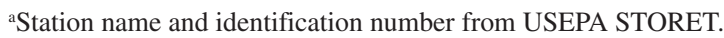

${ }^{\mathrm{b}}$ Station name and identification number from USGS NWIS. 


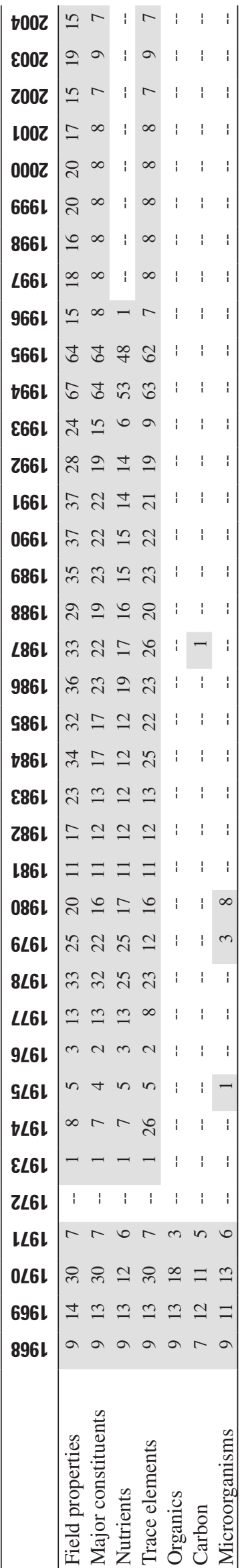

the park but upstream from the wastewater treatment plant. Although concentrations were fairly low, there were detectable levels of nutrients in nearly all the river samples. From 1987 to 1995 , nitrate concentrations ranged from 0.02 to $0.94 \mathrm{mg} / \mathrm{L}$ and orthophosphate concentrations ranged from 0.001 to 0.024 $\mathrm{mg} / \mathrm{L}$. Nitrate showed a strong seasonal pattern at site S1, with winter concentrations as much as five times higher than summer concentrations (fig. 13). Orthophosphate concentrations at site $\mathrm{S} 1$ also were elevated in winter relative to summer. Higher nutrient concentrations in winter probably are caused by greater contributions of nutrient-enriched ground water to streamflow coupled with decreased biological demand during colder winter months. Major sources of nitrogen and phosphorus to the river upstream from sites S1 and S6 include point sources such as the municipal wastewater treatment plant in the town of Butte, and seepage from septic systems and nonpoint sources such as animal wastes and irrigated agricultural land (http://www.tristatecouncil.org/documents/02vnrp_eval. $p d f$, accessed June 2006).

Trace elements detected in river water included arsenic, cadmium, copper, iron, lead, manganese, and zinc, which are metals associated with the Superfund mine tailings (Lambing, 1991). All of the main-stem samples had detectable levels of dissolved arsenic, which ranged from 6.0 to $39 \mu \mathrm{g} / \mathrm{L}$, and dissolved copper, which ranged from 3 to $120 \mu \mathrm{g} / \mathrm{L}$ (table 16). Dissolved cadmium and lead were present in lower concentrations, and were detected in about 25 percent of samples. The highest dissolved trace-element concentrations were for manganese, which ranged from 1 to $400 \mu \mathrm{g} / \mathrm{L}$ and had a median concentration of $50 \mu \mathrm{g} / \mathrm{L}$. The maximum dissolved copper (120 $\mu \mathrm{g} / \mathrm{L})$ and zinc $(230 \mu \mathrm{g} / \mathrm{L})$ concentrations were measured during an intense rainstorm on July 12, 1989, that flushed metal salts from tailings into the river and caused a fishkill (Lambing, 1991). Trace-element concentrations in the Clark Fork River at S3 showed strong seasonal patterns that were opposite to those observed for major constituents. For example, the highest concentrations of dissolved arsenic and copper concentrations at site $\mathrm{S} 3$ occurred during spring runoff, when major dissolved constituents were at a minimum (fig. 13). One possible explanation for this pattern is greater amounts of suspended sediment in the river during high-flow conditions (Lambing, 1991). Elevated suspended sediment, which often exceeds $50 \mathrm{mg} / \mathrm{L}$ during spring runoff, is caused by erosion of mine tailings from stream banks and floodplain deposits. Another potential source of dissolved metals is flushing of salts that accumulate in tailings and contaminated soils during the winter months. In addition to seasonality, metal concentrations also showed substantial diel cycles in the Clark Fork River (Brick and Moore, 1996). Daily variations in dissolved manganese and zinc concentrations and $\mathrm{pH}$ at site $\mathrm{S} 6$ are shown in figure 14. Metal concentrations increased during the night, reaching the highest concentrations at sunrise. Concentrations began to decrease during the late morning, reaching the lowest concentrations during late afternoon. A similar diel pattern has been documented in a wide variety of $\mathrm{pH}$ neutral streams in the Northern Rocky Mountains, which typically is attributed to sorption of 
Table 16. Summary of selected water-quality data for main-stem sites on the Clark Fork River near Grant-Kohrs Ranch National Historic Site, Montana, 1980 to 2004.

[no. number; chronic aquatic-life (and human-health) standards from www.deq.state.mt.us/wqinfo/Standards/Index.asp; >, greater than; --, not reported; ${ }^{\circ} \mathrm{C}$, degrees Celsius; $\mathrm{mg} / \mathrm{L}$, milligrams per liter; $\mu \mathrm{S} / \mathrm{cm}$, microsiemens per centimeter at $25^{\circ} \mathrm{C} ; \mathrm{CaCO}_{3}$, calcium carbonate; $\mathrm{N}$, nitrogen; $\mathrm{P}$, phosphorous; $\mu \mathrm{g} / \mathrm{L}$, micrograms per liter]

\begin{tabular}{|c|c|c|c|c|c|}
\hline Constituent or property & $\begin{array}{l}\text { No. analyses } \\
\text { (no. censored) }\end{array}$ & $\begin{array}{l}\text { Minimum } \\
\text { value }\end{array}$ & $\begin{array}{c}\text { Median } \\
\text { value }\end{array}$ & $\begin{array}{l}\text { Maximum } \\
\text { value }\end{array}$ & $\begin{array}{c}\text { Aquatic-life } \\
\text { (human-health) } \\
\text { standard }\end{array}$ \\
\hline \multicolumn{6}{|c|}{ Field properties } \\
\hline Temperature, water $\left({ }^{\circ} \mathrm{C}\right)$ & 637 & 0.0 & 9.6 & 26.5 & -- \\
\hline Oxygen, dissolved (mg/L) & 94 & 6.2 & 10.4 & 12.4 & $>4.0$ \\
\hline $\mathrm{pH}$ (standard units) & 351 & 6.70 & 8.27 & 8.91 & a $6.5-9.0$ \\
\hline Specific conductance $(\mu \mathrm{S} / \mathrm{cm})$ & 534 & 208 & 524 & 1,890 & -- \\
\hline \multicolumn{6}{|c|}{ Major constituents } \\
\hline Alkalinity $\left(\mathrm{mg} / \mathrm{L}\right.$ as $\left.\mathrm{CaCO}_{3}\right)$ & 304 & 7.0 & 142 & 199 & -- \\
\hline Calcium, dissolved (mg/L) & 418 & 28 & 72 & 134 & -- \\
\hline Chloride, dissolved (mg/L) & 308 & .5 & 8.6 & 18 & -- \\
\hline Fluoride, dissolved (mg/L) & 82 & .0 & .7 & 1.0 & $(4.0)$ \\
\hline Magnesium, dissolved (mg/L) & 413 & 4.7 & 15 & 27 & -- \\
\hline Potassium, dissolved (mg/L) & 90 & 1.9 & 3.7 & 6.7 & -- \\
\hline Silica, dissolved (mg/L) & 131 & 9.1 & 13 & 21 & -- \\
\hline Sodium, dissolved (mg/L) & 307 & 3.5 & 19 & 28 & -- \\
\hline Sulfate, dissolved (mg/L) & 215 & 11 & 120 & 350 & -- \\
\hline \multicolumn{6}{|c|}{ Nutrients } \\
\hline Ammonia, dissolved (mg/L as $\mathrm{N})$ & 90 & 0.00 & 0.01 & 0.23 & ${ }^{\mathrm{b}} 3.15$ \\
\hline Nitrate, dissolved (mg/L as N) & 185 & .02 & .18 & .94 & c.3 (10) \\
\hline Orthophosphate, dissolved (mg/L as P) & 89 & .001 & .006 & .024 & ${ }^{\mathrm{c}} .02$ \\
\hline \multicolumn{6}{|c|}{ Trace elements } \\
\hline Arsenic, dissolved $(\mu \mathrm{g} / \mathrm{L})$ & 146 & 6 & 13 & 39 & (10) \\
\hline Cadmium, dissolved $(\mu \mathrm{g} / \mathrm{L})$ & $144(108)$ & ${ }^{\mathrm{d}} .02$ & .1 & 5 & $(5)$ \\
\hline Copper, dissolved ( $\mu \mathrm{g} / \mathrm{L})$ & 251 & 3 & 8 & 120 & $(1,300)$ \\
\hline Iron, dissolved $(\mu \mathrm{g} / \mathrm{L})$ & $247(25)$ & $\mathrm{d}_{3}$ & 13 & 190 & $(\mathrm{e} 300)$ \\
\hline Lead, dissolved $(\mu \mathrm{g} / \mathrm{L})$ & $148(108)$ & ${ }^{\mathrm{d}} .04$ & 6 & 50 & (15) \\
\hline Manganese, dissolved ( $\mu \mathrm{g} / \mathrm{L})$ & 243 & 1 & 50 & 400 & $\left({ }^{e} 50\right)$ \\
\hline Zinc, dissolved ( $\mu \mathrm{g} / \mathrm{L})$ & 247 & .9 & 11 & 230 & $(2,000)$ \\
\hline Aluminum, total $(\mu \mathrm{g} / \mathrm{L})$ & 122 & 18 & 100 & 1,129 & f87 \\
\hline Arsenic, total $(\mu \mathrm{g} / \mathrm{L})$ & 321 & 2 & 4 & 240 & 150 \\
\hline Cadmium, total $(\mu \mathrm{g} / \mathrm{L})$ & $287(89)$ & ${ }^{\mathrm{d}} .06$ & 1 & 8 & ${ }^{\mathrm{b}} .5$ \\
\hline Copper, total $(\mu \mathrm{g} / \mathrm{L})$ & 442 & 2 & 25 & 1,500 & $\mathrm{~b}_{20}$ \\
\hline Iron, total $(\mu \mathrm{g} / \mathrm{L})$ & 434 & 27 & 310 & 29,000 & 1,000 \\
\hline Lead, total $(\mu \mathrm{g} / \mathrm{L})$ & $291(8)$ & ${ }^{\mathrm{d}} .5$ & 6 & 200 & ${ }^{\mathrm{b}} 10$ \\
\hline Manganese, total $(\mu \mathrm{g} / \mathrm{L})$ & 417 & 8 & 120 & 4,600 & -- \\
\hline Zinc, total $(\mu \mathrm{g} / \mathrm{L})$ & $444(2)$ & $\mathrm{d}_{5}$ & 40 & 1,700 & b260 \\
\hline
\end{tabular}

${ }^{a}$ Natural $\mathrm{pH}$ outside this range must be maintained.

${ }^{\mathrm{b}}$ Table value standard calculated for a hardness of $250 \mathrm{mg} / \mathrm{L}$ for trace elements or $\mathrm{pH}$ of 8.3 at $20{ }^{\circ} \mathrm{C}$ for ammonia.

${ }^{c}$ For reduction of nuisance algae growth, standards are for total nitrogen and total phosphorus.

${ }^{\mathrm{d}}$ Minimum reported value less than minimum censored value.

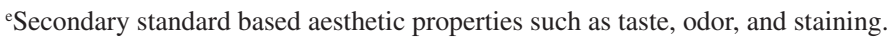

fDissolved concentration. 

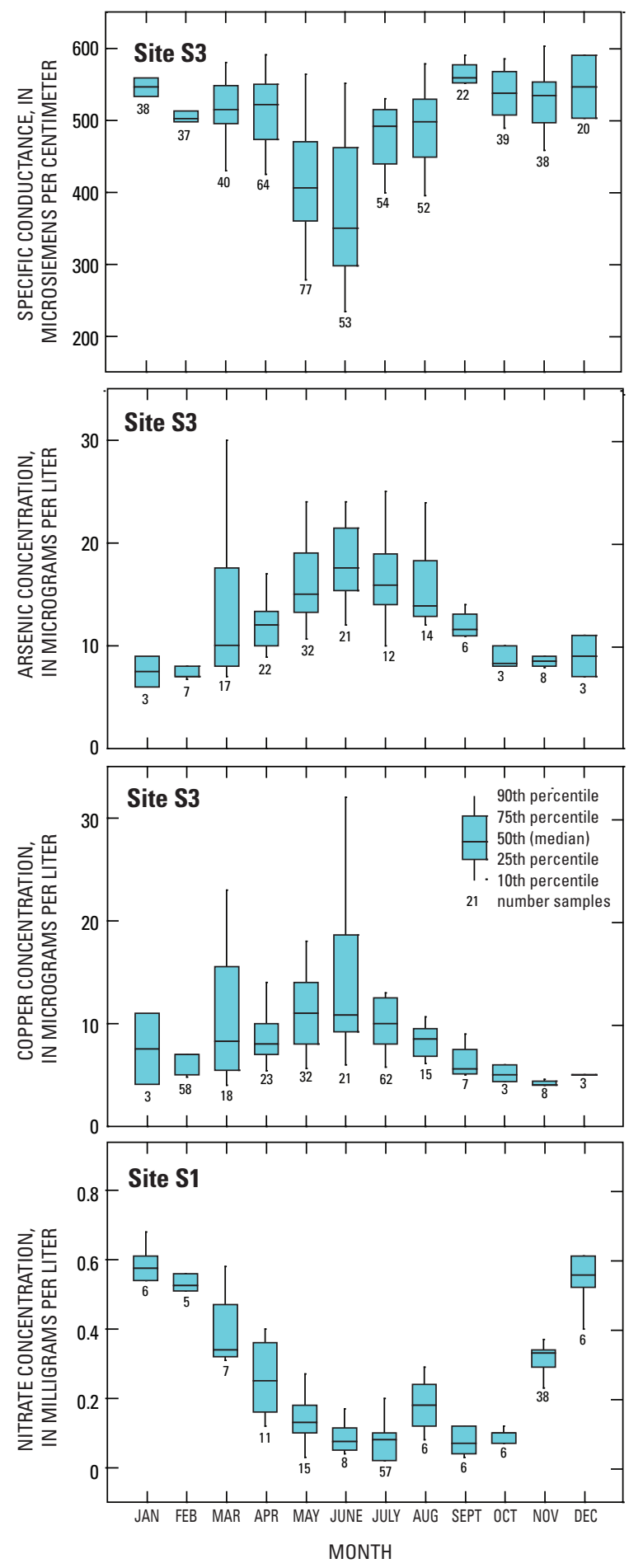

Figure 13. Seasonal variation in water quality at sites $\mathrm{S} 1$ and S3 on the Clark Fork River near Grant-Kohrs Ranch National Historic Site, Montana, 1982-2004.

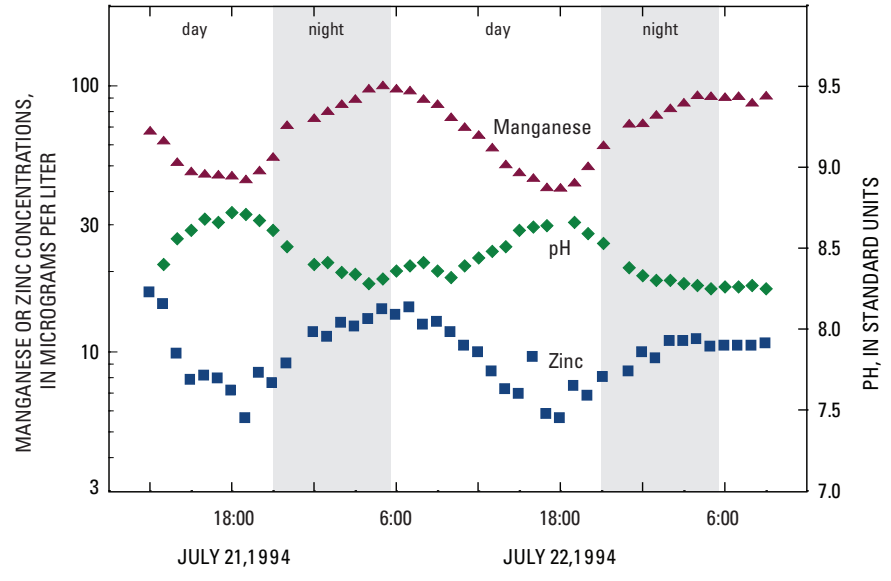

Figure 14. Diurnal variations in $\mathrm{pH}$, manganese, and zinc at Clark Fork River (S6) in Grant-Kohrs Ranch National Historic Site, Montana, July 21-23, 1994.

metals between stream water and streambed sediments and aquatic plants (Nimick, 2003). Daily cycles in stream-water temperature and $\mathrm{pH}$ seem to control the amount of each metal that is adsorbed or desorbed (Nimick, 2003).

The Clark Fork River at sites S1 and S3 had sufficient data to test for temporal trends in water quality. At site S3, trends were tested for discharge, $\mathrm{pH}$, calcium, magnesium, and dissolved arsenic, copper, iron, manganese, and zinc concentrations from 1989 to 2004 using four seasons per year (table 17). A statistically significant upward trend was detected for $\mathrm{pH}$, and a downward trend was detected for zinc at site S3. The magnitude and significance of the $\mathrm{pH}$ trend were the same between unadjusted and flow-adjusted concentrations, indicating patterns in discharge were not driving the trend. The flow model for zinc was not statistically significant; therefore flowadjusted concentrations were not tested. Total recoverable trace-element concentrations at this site also were tested, but none of the constituents (including zinc) had significant trends. At site S1, discharge and major constituents were tested from 1980 to 1992 using 12 seasons per year (table 17). A strong downward trend was detected in streamflow, which reflects an extended period of drought during the late 1980s. A statistically significant upward trend was detected in alkalinity; however, the trend changed direction and decreased in significance after flow-adjustment, indicating it was largely driven by the downward trend in streamflow. By contrast, there was a significant downward trend in the flow-adjusted calcium concentrations that was not detected in the unadjusted concentrations perhaps because it was offset by the decline in streamflows. A strong downward trend was detected in unadjusted sulfate concentrations but the flow-adjusted concentrations were not tested because the flow model was weak. The magnitudes of the calcium $(-1.4 \mathrm{mg} / \mathrm{L} / \mathrm{yr})$ and sulfate $(-4.0 \mathrm{mg} / \mathrm{L} / \mathrm{yr})$ trends were fairly large and were similar on an equivalent basis [-70 microequivalents per liter per year ( $\mu \mathrm{eq} / \mathrm{L} / \mathrm{yr})$ for calcium compared to $-83 \mu \mathrm{eq} / \mathrm{L} / \mathrm{yr}$ for sulfate] indicating they reflect real changes in stream-water quality. Because sulfate and 
Table 17. Results of the seasonal Kendall test for trends in streamflow and unadjusted and flow-adjusted constituent concentrations for two stations on the Clark Fork River near Grant-Kohrs Ranch National Historic Site, Montana.

$[--$, not calculated or flow model not statistically significant at $\mathrm{p} \leq 0.1 ; \mathrm{ft} 3 \mathrm{~s} / \mathrm{yr}$, cubic feet per second per year; $\mathrm{mg} / \mathrm{L} / \mathrm{yr}$, milligrams per liter per year; $\mu \mathrm{g} / \mathrm{L} / \mathrm{yr}$, micrograms per liter per year; trends in bold are significant at $\mathrm{p} \leq 0.01$ ]

\begin{tabular}{|c|c|c|c|c|c|}
\hline \multirow[b]{2}{*}{ Parameter } & \multicolumn{2}{|c|}{ Unadjusted } & \multicolumn{2}{|c|}{ Flow-adjusted } & \multirow[b]{2}{*}{ Period of record } \\
\hline & trend & p-value & trend & p-value & \\
\hline \multicolumn{6}{|c|}{ Clark Fork at Deer Lodge (S3 from fig. 10) } \\
\hline Discharge $\left(\mathrm{ft}^{3} / \mathrm{s} / \mathrm{yr}\right)$ & -0.67 & 0.709 & -- & -- & 1989-2004 \\
\hline pH (standard units/yr) & .01 & .007 & 0.01 & 0.007 & 1989-2004 \\
\hline Calcium (mg/L/yr) & .25 & .375 & -.29 & .170 & 1989-2004 \\
\hline Magnesium (mg/L/yr) & -.01 & .651 & .04 & .511 & 1989-2004 \\
\hline Arsenic, dissolved ( $\mu \mathrm{g} / \mathrm{L} / \mathrm{yr})$ & .04 & .512 & .06 & .484 & 1989-2004 \\
\hline Copper, dissolved ( $\mu \mathrm{g} / \mathrm{L} / \mathrm{yr})$ & -.19 & .025 & -.09 & .355 & 1989-2004 \\
\hline Iron, dissolved $(\mu \mathrm{g} / \mathrm{L} / \mathrm{yr})$ & .00 & .770 & -- & -- & 1989-2004 \\
\hline Manganese, dissolved ( $\mu \mathrm{g} / \mathrm{L} / \mathrm{yr})$ & .13 & .647 & -- & -- & 1989-2004 \\
\hline Zinc, dissolved ( $\mu \mathrm{g} / \mathrm{L} / \mathrm{yr})$ & -.52 & .008 & -- & -- & 1989-2004 \\
\hline \multicolumn{6}{|c|}{ Clark Fork River (S1 from fig. 10) } \\
\hline Discharge $\left(\mathrm{ft}^{3} / \mathrm{s} / \mathrm{yr}\right)$ & -20.4 & 0.000 & -- & -- & 1980-1992 \\
\hline Alkalinity (mg/L/yr) & 2.0 & .008 & -1.1 & 0.022 & 1980-1992 \\
\hline Calcium (mg/L/yr) & -.63 & .120 & -1.4 & .008 & 1980-1992 \\
\hline Chloride (mg/L/yr) & .20 & .039 & -.04 & .399 & 1980-1992 \\
\hline Magnesium (mg/L/yr) & .08 & .489 & -.18 & .072 & 1980-1992 \\
\hline Sodium (mg/L/yr) & .29 & .060 & -.11 & .307 & 1980-1992 \\
\hline Sulfate (mg/L/yr) & -4.0 & .009 & -- & -- & 1980-1992 \\
\hline
\end{tabular}

zinc are associated with mine wastes, it is likely the observed trends in water quality reflect remediation and restoration efforts in the headwater areas of the Clark Fork drainage during the 1980s and early 1990s (http://www.epa.gov/region8/ superfund/mtsf.html, accessed June 2006); however, a detailed analysis of these trends with respect to specific remediation efforts is beyond the scope of this report. Continued monitoring will be important for assessing future remediation activities related to Superfund, and in particular, those activities that might occur inside the boundaries of GRKO.

Comparison to Water-Quality Standards. Water-use classifications and water-quality standards for all stream segments in Montana are established by the MTDEQ (http://www. deq.state.mt.us/wqinfo/Standards/Index.asp, accessed June 2006). The main stem of the Clark Fork River in the vicinity of GRKO (Clark Fork River downstream from Cottonwood Creek) has a beneficial use classification of $\mathrm{C}-1$, which is suitable for bathing, swimming, and recreation; growth and propagation of salmonid fishes and associated aquatic life, waterfowl, and furbearers; and agricultural and industrial water supply. This reach of the Clark Fork River is on the most recent (2006) 303(d) list for the State of Montana (http://www. deq.state.mt.us/CWAIC, accessed June 2006) and is severely impaired for aquatic life and cold water fishery, and partially impaired for primary contact recreational use. The sources of the impairment are mill tailings, which have caused elevated copper, lead, and zinc concentrations, and agriculture and municipal point source discharge, which have resulted in elevated nitrogen, phosphorus, and sediment concentrations. In addition, discharge in the river during August and September is less than the minimum necessary for maintenance of a salmonid fishery. Contaminated sediments and siltation also have cause partial impairment for primary contact recreational use.

Aquatic-life and human-health standards established by the MTDEQ for surface water are compared to available water-quality data for the main-stem sites in table 16. Aquaticlife standards for copper, cadmium, lead, and zinc are dependent on hardness and standards were calculated using a median hardness of $250 \mathrm{mg} / \mathrm{L}$. Comparing total trace-element concentrations to aquatic-life standards indicates exceedances for all the trace elements listed, with copper exceeding the standards the most frequently (60 percent of samples). Comparing dissolved trace-element concentrations to human-health standards indicates numerous exceedances for arsenic and manganese, and a few for lead. This reach of the river, however, is classified as $\mathrm{C}-1$ and drinking water is not a beneficial use. 
Standards for total nitrogen and phosphorus in the Clark Fork River have been established by the VNRP to reduce nuisance algae growth in the Clark Fork River downstream from Warm Springs in summer and to restore nutrient-impaired water uses along the river. (http://www.ci.missoula.mt.us/ wastewater/VNRP.htm, accessed June 2006). The numeric standards are $0.02 \mathrm{mg} / \mathrm{L}$ for total phosphorus and $0.3 \mathrm{mg} / \mathrm{L}$ for total nitrogen, which are applicable from June 21 through September 21 (http://www.deq.state.mt.us/dir/Legal/Chapters/ CH30-06.pdf, accessed June 2006). Assuming most nitrogen is present as dissolved nitrate, more than 40 percent of the nitrate concentrations in the main-stem samples exceeded the total nitrogen standard; however, none of the exceedances occurred during the summer months. Total phosphorus was not measured; however, 5 percent of samples had orthophosphate concentrations that exceeded the total phosphorus standard (20 $\mu \mathrm{g} / \mathrm{L}$ ) and, similar to nitrate, none of the exceedances occurred during summer months.

\section{Ground-Water Quality}

Water-quality data are available for 79 samples collected at 20 ground-water wells in or immediately adjacent to GRKO (table 18, fig. 10). Sites G1 to G4 were sampled between 1985 and 1989 as part of a cooperative study between the USGS and Montana Bureau of Mines and Geology to describe hydrologic conditions along the upper Clark Fork (Nimick, 1993). Samples collected at these wells were analyzed at the Montana Bureau of Mines and Geology Analytical Laboratory (http:// www.mbmg.mtech.edu/analytical.htm, accessed June 2006). Site G5 was sampled and analyzed by the Montana Bureau of Mines and Geology in 1980 and sites G6 to G21 were installed and sampled in 2000 by the University of Montana as a cooperative project with the NPS to investigate metal-contaminated ground water in GRKO (Moore and Woessner, 2000).

Water-quality results for the 79 ground-water samples from GRKO are summarized in table 19. All of the samples had moderately high levels of major constituents with dissolved solids (calculated) ranging from 213 to $536 \mathrm{mg} / \mathrm{L}$. Calcium was the dominant cation and bicarbonate was the dominant anion (based on $\mathrm{pH}$ and alkalinity) reflecting dissolution of carbonates rocks that occur as fragments in the alluvial deposits. Although sulfate was less abundant than bicarbonate, it ranged from 16 to $101 \mathrm{mg} / \mathrm{L}$ and accounted for as much as 35 percent of the total anion charge in some of the wells. Because there are no large sources of sulfate in the bedrock and alluvium, most sulfate in ground water likely is from oxidation of sulfide minerals in mine tailings that contaminate the floodplain. Wells with the highest sulfate concentrations also had elevated arsenic concentrations, which supports the idea that sulfate is derived from mine wastes. Another possible source of sulfate (and chloride) is wastewater effluent used to irrigate hayfields during the summer as part of the VNRP. Johnson (2002) reported concentrations as high as $47 \mathrm{mg} / \mathrm{L}$ for sulfate and $28 \mathrm{mg} / \mathrm{L}$ for chloride from effluent collected at sprinkler heads during 2000. None of the ground-water sites had a sufficient period of record to test for changes in water quality with time.

Despite agricultural activities in the park and tailings deposits in the floodplain, concentrations of nutrients and trace elements in the shallow ground-water samples generally were low. Nitrate concentrations ranged from less than 0.01 to 2.2 and were greater than $1.0 \mathrm{mg} / \mathrm{L}$ in only 15 percent of the samples. Nimick (1993) suggested that nitrate concentrations above $1.0 \mathrm{mg} / \mathrm{L}$ in the upper Clark Fork River basin probably indicate a nitrate source that is related to agricultural activities. In GRKO, an additional source of nitrate to ground water is treated sewage effluent used for irrigation, although, an NPS study in 2000 did not find significant differences between nitrate concentrations in background wells compared to wells in irrigated fields (Johnson, 2002). Of the 10 trace elements reported, dissolved concentrations commonly were close to or less than the laboratory reporting levels. The most frequent detections were for aluminum (48), arsenic (32 percent), copper (32 percent), iron (97 percent), manganese (97 percent), and zinc (100 percent). A likely source of arsenic, copper, and zinc is leaching of mine tailings that contaminate the floodplain sediments and soils. Because these wells are along the floodplain, metals also could come from percolation of contaminated river water into the underlying alluvial aquifer.

Comparison to Water-Quality Standards. Although ground-water wells in the park are not used as a water supply, samples were compared to human-health standards established by the MTDEQ (http://www.deq.state.mt.us/wqinfo/Standards/ Index.asp, accessed June 2006). Primary drinking-water standards are developed for human health, and secondary standards are based on aesthetic properties such as taste, odor, and staining. Arsenic concentrations in 12 samples (ranging from 11 to $39 \mu \mathrm{g} / \mathrm{L}$ ) from four wells exceeded the human health standard of $10 \mu \mathrm{g} / \mathrm{L}$. None of the other trace-element concentrations exceeded the human-health standards, although one sample exceeded the secondary standard for iron and 18 exceeded the secondary standard for manganese. Elevated manganese concentrations probably are related to leaching of metals from tailings deposits in the floodplain or infiltration of river water into the alluvial aquifer.

\section{Summary and Suggestions for Vital Signs Monitoring}

Data for surface-water quality at GRKO are available for 881 samples collected at 7 main-stem sites on the Clark Fork River and 6 tributary streams from 1968 to 2004. The majority of results for these 881 samples were field properties (24 percent), and major-constituent ( 24 percent), nutrient (9 percent), and trace-element (30 percent) analyses. One-half of the water-quality samples were collected at the Clark Fork River at Deer Lodge (site S3), which has been sampled intensively since 1982. A streamflow-gaging station has been operated by the USGS at this station since 1979. Water in the Clark 
Table 18. Ground-water sampling sites near Grant-Kohrs Ranch National Historic Site, Montana.

[no., number; well depth in meters; GKW, Grant-Kohrs well; MW, monitoring well; station names beginning with 08 from USGS NWIS; station names beginning with GKW or MW from Moore and Woessner (2000)]

\begin{tabular}{|c|c|c|c|c|c|c|c|}
\hline $\begin{array}{l}\text { Site no. } \\
\text { (fig. 10) }\end{array}$ & Identification no. & Station name & Latitude & Longitude & Well depth & $\begin{array}{l}\text { Period of } \\
\text { record }\end{array}$ & $\begin{array}{c}\text { No. of } \\
\text { samples }\end{array}$ \\
\hline G1 & 462352112442201 & 08N09W33CCDD01 & $46^{\circ} 23^{\prime} 51^{\prime \prime}$ & $112^{\circ} 44^{\prime} 25^{\prime \prime}$ & 50.0 & 1985 & 1 \\
\hline $\mathrm{G} 2$ & 462429112444301 & 08N09W32AADD01 & $46^{\circ} 24^{\prime} 28^{\prime \prime}$ & $112^{\circ} 44^{\prime} 46^{\prime \prime}$ & 2.9 & $1986-87$ & 2 \\
\hline G3 & 462429112444302 & 08N09W32AADD02 & $46^{\circ} 24^{\prime} 28^{\prime \prime}$ & $112^{\circ} 44^{\prime} 46^{\prime \prime}$ & 18.1 & 1987,89 & 2 \\
\hline G4 & 462457112434901 & 08N09W28DBDC01 & $46^{\circ} 24^{\prime} 56^{\prime \prime}$ & $112^{\circ} 43^{\prime} 52^{\prime \prime}$ & 18.0 & 1985 & 1 \\
\hline G5 & 462430112441601 & 08N09W33BAC 01 & $46^{\circ} 24^{\prime} 29^{\prime \prime}$ & $112^{\circ} 44^{\prime} 19^{\prime \prime}$ & 1.8 & 1980 & 1 \\
\hline G6 & 462528112445300 & GKW-1 & $46^{\circ} 25^{\prime} 04^{\prime \prime}$ & $112^{\circ} 44^{\prime} 39^{\prime \prime}$ & 10.2 & 2000 & 5 \\
\hline G7 & 462454112450700 & GKW-2 & $46^{\circ} 25^{\prime} 22^{\prime \prime}$ & $112^{\circ} 44^{\prime} 06^{\prime \prime}$ & 13.1 & 2000 & 4 \\
\hline G8 & 462439112445900 & GKW-3 & $46^{\circ} 24^{\prime} 53^{\prime \prime}$ & $112^{\circ} 45^{\prime} 06^{\prime \prime}$ & 3.8 & 2000 & 2 \\
\hline G9 & 462426112450900 & GKW-4 & $46^{\circ} 24^{\prime} 39^{\prime \prime}$ & $112^{\circ} 44^{\prime} 59^{\prime \prime}$ & 10.2 & 2000 & 6 \\
\hline G10 & 462408112444100 & GKW-5 & $46^{\circ} 24^{\prime} 26^{\prime \prime}$ & $112^{\circ} 45^{\prime} 09^{\prime \prime}$ & 5.8 & 2000 & 3 \\
\hline G12 & 462410112442100 & GKW-7 & $46^{\circ} 24^{\prime} 09^{\prime \prime}$ & $112^{\circ} 45^{\prime} 17^{\prime \prime}$ & 4.3 & 2000 & 5 \\
\hline G13 & 462428112443600 & GKW-8 & $46^{\circ} 24^{\prime} 09^{\prime \prime}$ & $112^{\circ} 44^{\prime} 20^{\prime \prime}$ & 4.4 & 2000 & 6 \\
\hline G14 & 462446112442700 & GKW-9 & $46^{\circ} 24^{\prime} 28^{\prime \prime}$ & $112^{\circ} 44^{\prime} 36^{\prime \prime}$ & 3.0 & 2000 & 6 \\
\hline G15 & 462525112442100 & GKW-10 & $46^{\circ} 25^{\prime} 28^{\prime \prime}$ & $112^{\circ} 44^{\prime} 53^{\prime \prime}$ & 5.8 & 2000 & 4 \\
\hline G16 & 462523112440600 & GKW-11 & $46^{\circ} 25^{\prime} 24^{\prime \prime}$ & $112^{\circ} 44^{\prime} 21^{\prime \prime}$ & 7.3 & 2000 & 4 \\
\hline G17 & 462453112434000 & MW1 & $46^{\circ} 25^{\prime} 37^{\prime \prime}$ & $112^{\circ} 44^{\prime} 07^{\prime \prime}$ & 8.7 & 2000 & 5 \\
\hline G18 & 462527112435200 & MW2 & $46^{\circ} 24^{\prime} 53^{\prime \prime}$ & $112^{\circ} 43^{\prime} 40^{\prime \prime}$ & 5.5 & 2000 & 5 \\
\hline G19 & 462502112442400 & MW3 & $46^{\circ} 25^{\prime} 26^{\prime \prime}$ & $112^{\circ} 43^{\prime} 51^{\prime \prime}$ & 4.4 & 2000 & 1 \\
\hline G20 & 462514112441200 & MW4 & $46^{\circ} 25^{\prime} 02^{\prime \prime}$ & $112^{\circ} 44^{\prime} 23^{\prime \prime}$ & 5.1 & 2000 & 8 \\
\hline G21 & 462539112440700 & MW5 & $46^{\circ} 25^{\prime} 13^{\prime \prime}$ & $112^{\circ} 44^{\prime} 12^{\prime \prime}$ & 4.9 & 2000 & 8 \\
\hline
\end{tabular}

Fork River is a calcium-bicarbonate-sulfate type, and major constituents primarily are derived from weathering of rock fragments in the alluvial fill except sulfate, which is released from oxidation of pyrite present in mine wastes. Arsenic, cadmium, copper, lead, manganese, and zinc, which are trace elements associated with mine wastes from historical mining activities, commonly were measured at detectable levels in the river. Nutrients in the river were mainly human-derived related to wastewater discharge and agriculture. Statistically significant downward trends were detected in dissolved calcium, sulfate, and zinc in the Clark Fork River that were attributed to remediation and restoration efforts during the 1980s and early 1990s. The Clark Fork River in the vicinity of GRKO has a beneficial-use classification of C-1 (suitable for bathing, swimming and recreation; growth and propagation of salmonid fishes and associated aquatic life, waterfowl, and furbearers; and agricultural and industrial water supply) and is listed as severely impaired for aquatic life and cold-water fishery and partially impaired for primary-contact recreational use. The sources of impairment are mine wastes, agriculture, and municipal wastewater discharge.

Data for ground-water quality at GRKO are available for 79 samples collected at 20 shallow ground-water wells from
1980 to 2000. The majority of results for these 77 samples were field properties (14 percent), and major-constituent (28 percent), nutrient ( 9 percent), and trace-element (39 percent) analyses. Most of the samples were collected in 2000 as part of a NPS ground-water study. Ground water is a calciumbicarbonate type with moderately high dissolved solids (213 to $536 \mathrm{mg} / \mathrm{L}$ ). Nutrient concentrations in ground water were low, indicating minimal effects from agricultural activities in the park. Samples from 4 of the 21 ground-water wells exceeded the human-health standard for arsenic, although none of the wells are used as a water supply.

Nutrient input from the sewage irrigation program and trace-element and sediment contamination related to mine tailings and their remediation are the primary water-quality issues for the reach of the Clark Fork River in GRKO. Based on a review of the historical data, the following suggestions are provided for consideration in designing a water-quality monitoring plan for GRKO.

- Maintain long-term water-quality monitoring at the Clark Fork River at Deer Lodge (USGS streamflowgaging station 12324200) and establish as a sentinel site. The site is sampled 10-20 times per year by the USGS for trace elements, sediment, and hardness in 
Table 19. Summary of selected water-quality data for ground-water sites in Grant-Kohrs Ranch National Historic Site, Montana, 1980 to 2000.

[no., number; human-health standards from www.deq.state.mt.us/wqinfo/Standards/Index.asp; <, less than; --, not reported; ${ }^{\circ} \mathrm{C}$, degrees Celsius; $\mathrm{mg} / \mathrm{L}$, milligrams per liter; $\mu \mathrm{S} / \mathrm{cm}$, microsiemens per centimeter at $25^{\circ} \mathrm{C} ; \mathrm{CaCO}_{3}$, calcium carbonate; $\mathrm{N}$, nitrogen; $\mathrm{P}$, phosphorous; $\mu \mathrm{g} / \mathrm{L}$, micrograms per liter; some nutrients and trace elements have multiple reporting limits]

\begin{tabular}{|c|c|c|c|c|c|c|}
\hline Constituent or property & No. sites & $\begin{array}{l}\text { No. analyses } \\
\text { (no. censored) }\end{array}$ & $\begin{array}{l}\text { Minimum } \\
\text { value }\end{array}$ & $\begin{array}{c}\text { Median } \\
\text { value }\end{array}$ & $\begin{array}{l}\text { Maximum } \\
\text { value }\end{array}$ & $\begin{array}{c}\text { Human-health } \\
\text { standard }\end{array}$ \\
\hline \multicolumn{7}{|c|}{ Field properties } \\
\hline Temperature, water $\left({ }^{\circ} \mathrm{C}\right)$ & 18 & 52 & 7.6 & 11.6 & 17.4 & -- \\
\hline Oxygen, dissolved (mg/L) & 3 & 3 & 4.6 & 5.6 & 6.4 & -- \\
\hline $\mathrm{pH}$ (standard units) & 19 & 53 & 6.45 & 7.31 & 7.68 & -- \\
\hline Specific conductance $(\mu \mathrm{S} / \mathrm{cm})$ & 19 & 53 & 242 & 457 & 799 & -- \\
\hline \multicolumn{7}{|c|}{ Major constituents } \\
\hline Alkalinity $\left(\mathrm{mg} / \mathrm{L}\right.$ as $\left.\mathrm{CaCO}_{3}\right)$ & 15 & 77 & 110 & 240 & 335 & -- \\
\hline Calcium, dissolved (mg/L) & 19 & 76 & 27 & 72 & 91 & -- \\
\hline Chloride, dissolved (mg/L) & 20 & 78 & 3.0 & 9.2 & 26 & -- \\
\hline Fluoride, dissolved (mg/L) & 5 & 7 & .1 & .7 & 1.0 & 4.0 \\
\hline Magnesium, dissolved (mg/L) & 19 & 76 & 4.4 & 23 & 31 & -- \\
\hline Potassium, dissolved (mg/L) & 19 & 76 & 1.6 & 4.5 & 8.4 & -- \\
\hline Silica, dissolved (mg/L) & 4 & 6 & 44 & 64 & 79 & -- \\
\hline Sodium, dissolved (mg/L) & 19 & 76 & 11 & 19 & 32 & -- \\
\hline Sulfate, dissolved (mg/L) & 20 & 78 & 16 & 46 & 101 & -- \\
\hline \multicolumn{7}{|c|}{ Nutrients } \\
\hline Nitrate, dissolved (mg/L as $\mathrm{N})$ & 19 & $77(12)$ & $<0.01$ & 0.5 & 2.2 & 10 \\
\hline Orthophosphate, dissolved ( $\mathrm{mg} / \mathrm{L}$ as $\mathrm{P}$ ) & 4 & $5(4)$ & $<.1$ & $<.1$ & .2 & -- \\
\hline Phosphorus, dissolved (mg/L as P) & 18 & $75(5)$ & $<.01$ & .05 & .16 & -- \\
\hline \multicolumn{7}{|c|}{ Trace elements } \\
\hline Aluminum, dissolved $(\mu \mathrm{g} / \mathrm{L})$ & 18 & 75 (39) & $<10$ & $<10$ & 280 & -- \\
\hline Arsenic, dissolved ( $\mu \mathrm{g} / \mathrm{L})$ & 18 & $75(51)$ & a3 & $<5$ & 39 & 10 \\
\hline Cadmium, dissolved ( $\mu \mathrm{g} / \mathrm{L})$ & 18 & $75(72)$ & $<1$ & $<1$ & 5 & 5 \\
\hline Chromium, dissolved $(\mu \mathrm{g} / \mathrm{L})$ & 18 & $75(71)$ & $<2$ & $<5$ & 5 & 100 \\
\hline Copper, dissolved $(\mu \mathrm{g} / \mathrm{L})$ & 18 & $75(51)$ & $<2$ & $<3$ & 7 & 1,300 \\
\hline Iron, dissolved $(\mu \mathrm{g} / \mathrm{L})$ & 17 & $72(2)$ & $<2.0$ & 36 & 707 & b300 \\
\hline Lead, dissolved ( $\mu \mathrm{g} / \mathrm{L})$ & 17 & $73(70)$ & $<10$ & $<10$ & $<40$ & 15 \\
\hline Manganese, dissolved ( $\mu \mathrm{g} / \mathrm{L})$ & 17 & $72(2)$ & $<1$ & 13 & 605 & $50^{2}$ \\
\hline Zinc, dissolved ( $\mu \mathrm{g} / \mathrm{L})$ & 18 & 75 & 1 & 3 & 120 & 2,000 \\
\hline
\end{tabular}

${ }^{a}$ Minimum reported value less than minimum censored value.

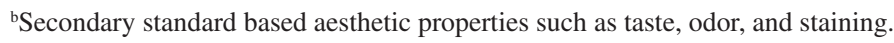


support of remediation efforts at the Clark Fork Superfund site. The addition of analyses for nutrients, major constituents, and bacteria could be considered. There is a USGS streamflow-gaging station at this site that has been in operation since 1979 .

- Establish a second water-quality monitoring site downstream from GRKO for periodic monitoring of nutrients, trace elements, sediment, and bacteria.

- Initiate routine monitoring of water levels and water quality (nutrients and trace elements) at a subset of shallow ground-water wells in the park. This information would be useful for monitoring the effects of the sewage irrigation program as well as future remediation efforts related to Superfund on ground-water resources.

\section{Great Sand Dunes National Park and Preserve}

\section{Environmental Setting and Geology}

Great Sand Dunes National Park and Preserve (GRSA) is located along the eastern edge of the broad, flat San Luis Valley in south-central Colorado (fig. 15). The covers 168 $\mathrm{km}^{2}$ and ranges in elevation from 2,293 $\mathrm{m}$ at the edge of the San Luis valley up to 4,123 m along the crest of the Sangre de Cristo Mountains, which form the eastern park boundary. The predominant feature of the park is a $63 \mathrm{~km}^{2}$ dune field in which some of the dunes are as much as $230 \mathrm{~m}$ high. Vegetation surrounding the dune field is dominated by grassland and shrub communities (Britten and others, 2006). East of the dunes, the lower flanks of the Sangre de Cristo Mountains are covered by pinion pine/juniper woodlands that grade into spruce/fir/aspen forests at higher elevations. The climate is characterized by cold winters with hot dry summers. Mean monthly air temperatures range from $-6{ }^{\circ} \mathrm{C}$ in January to $15^{\circ} \mathrm{C}$ in August at the park headquarters (http://www.wrcc.dri.edu/ summary/climsmco.html, accessed July 2006). Annual average precipitation at the park headquarters is $26 \mathrm{~cm}$, most of which falls during afternoon thundershowers in July, August, and September. Higher elevation areas in the eastern part of the park receive substantial snowfall that accumulates in a seasonal snowpack between November and April.

Bedrock in the Sangre de Cristo Mountains to the east of the dunes is Precambrian gneiss and micaceous schist (http://www2.nature.nps.gov/geology/parks/grsa/, accessed July 2006). Flat lying areas west of the mountains, including the dunes, are underlain by the Quaternary Alamosa Formation, which consists of unconsolidated gravel, sand, clay, and conglomerate. In the dune field itself, eolian deposits are subdivided into three provinces: the dune field, which is an area of actively forming reversing and star dunes; the sand sheet, which is composed of vegetation-stabilized dunes; and the Sabkha, which is a playa area where sand deposits are hardened by evaporate minerals (Chatman and others, 1997).
Land-use activities in GRSA are related to visitor use including the park headquarters and administrative area, a visitor center, an 88-site campground and recreational vehicle dump station, four picnic areas, and seasonal residences (Chatman and others, 1997). Some of the most intense visitor use is in a public wading area in the lower reach of Medano Creek. Land adjacent to the park mostly is public-owned and largely undeveloped. Water for park facilities is obtained from four ground-water wells located in the vicinity of park headquarters. Wastewater is treated at various locations in the park through septic tank-leach field combinations.

\section{Water Resources}

The two primary drainages in GRSA are Sand Creek, which flows around the northwest side of the dunes, and Medano Creek, which flows around the southeast side (fig. 15). The streams play a vital role in maintaining the dune fields because they deposit sand on the upwind side of the dune field where it is transported back to the dunes by prevailing winds (Chatman and others, 1997). Both streams begin high in the Sangre de Cristo Mountains and are fed primarily by snowmelt. As they flow around the edge of the dune field they begin losing water through the sand into a shallow unconfined ground-water aquifer. All the water in both creeks, except what is lost to evapotranspiration, eventually infiltrates into the unconfined aquifer, which flows under the dunes and eventually discharges at Big Spring and Little Spring Creeks on the west side of the dune field. Ground-water dating using chlorofluorocarbons indicates it takes more than 60 years for the ground water to flow under the dune field (Rupert and Plummer, 2004). A deeper confined aquifer, which also underlies the dune field, is separated from the unconfined aquifer by a 2.4-m thick clay layer at a depth of $90 \mathrm{~m}$ (Rupert and Plummer, 2004). There are insufficient data to determine whether or not the unconfined and confined aquifers are hydrologically connected (Rupert and Plummer, 2004). A more complete description of the surface- and ground-water hydrology of GRSA can be found in Chatman and others (1997).

The USGS operated a streamflow-gaging station on Mosca Creek (USGS station 08234200) a tributary of Medano Creek from 1967 to1970 (fig. 15). Streamflow-gaging stations have been operated on the two major perennial streams, Sand Creek (station SANDUNCO) and Medano Creek (station MEDSANCO), since 1992 (fig. 15). The gaging stations are operated by the Colorado Division of Water Resources for the NPS as part of a Federal water rights agreement. The range of daily streamflows by month at Medano Creek (station MEDSANCO) is shown in figure 16 . Streamflow typically peaks in late May to early June during spring snowmelt, and the lowest streamflows are in fall and winter (November to February) when flow is sustained primarily by ground water. Afternoon thundershowers contribute to streamflow through the summer months (July to September) and can cause flash flooding. The NPS also has weirs on 10 other streams or springs in the park 


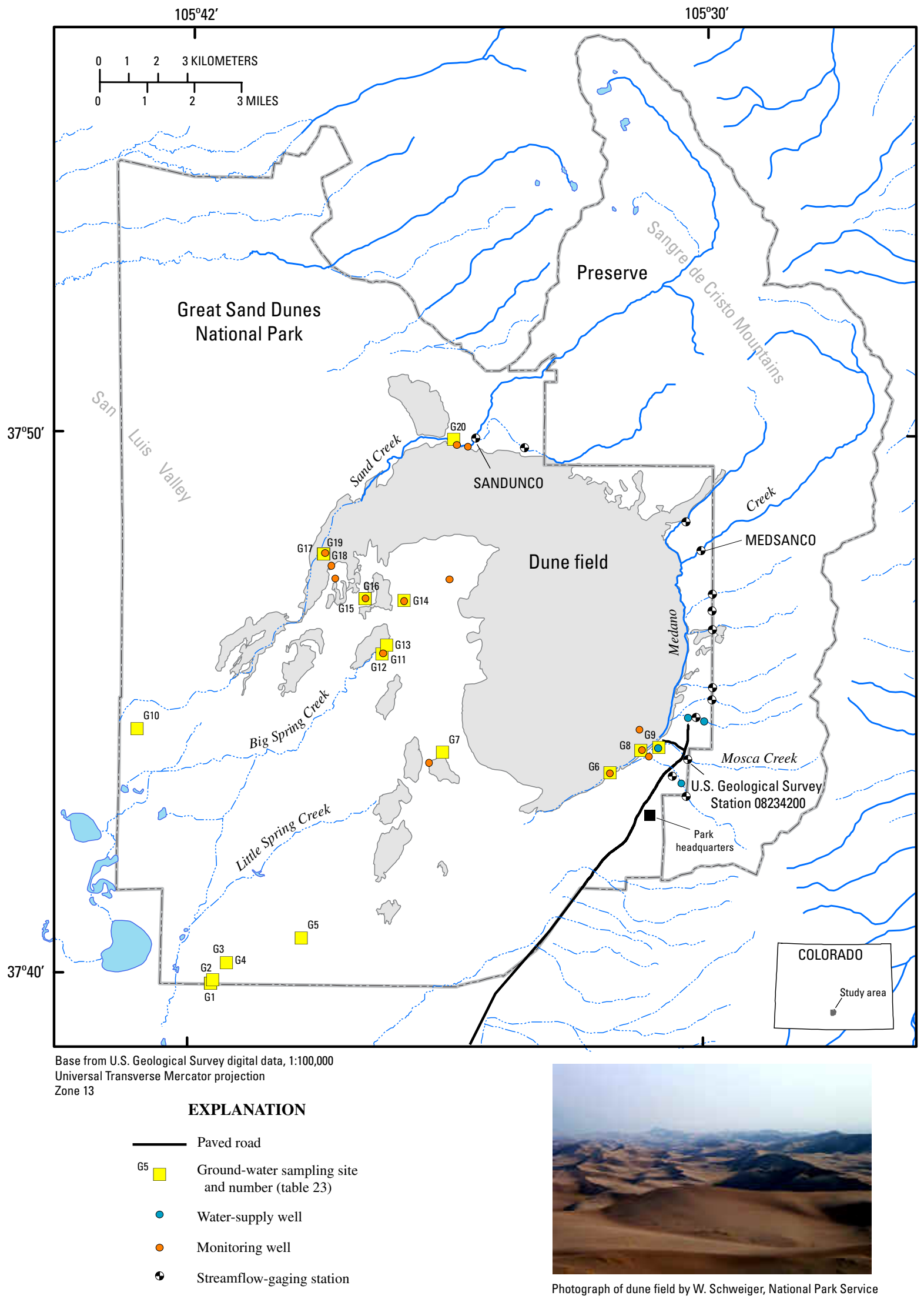

Figure 15. Location of streamflow-gaging stations and ground-water wells in Great Sand Dunes National Park and Preserve, Colorado. 


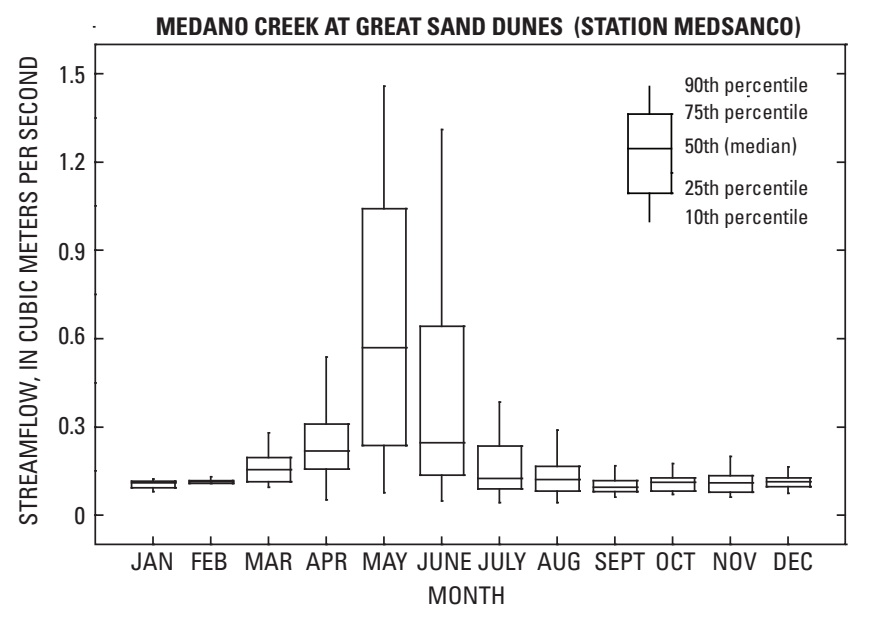

Figure 16. Daily streamflow range at Medano Creek (station MEDSANCO) in Great Sand Dunes National Park and Preserve, Colorado, 1992-2004.

that have water rights. Manual measurements have been made periodically by the NPS at these sites since 1992 .

The NPS initiated a ground-water monitoring program in 1990 and has installed 22 monitoring wells ranging from 2.4 to $120 \mathrm{~m}$ deep. Locations of some of the NPS monitoring wells are shown in figure 15, and detailed descriptions of the wells are provided by Chatman and others (1997). The purpose of the original program was to monitor the effects of a proposed project to mine ground water from the San Luis Valley (Chatman and others, 1997). Water-level measurements are made manually at some of the wells and continuously at others; water-level data for the wells are stored in an NPS database (Andrew Valdez, National park Service, oral commun., 2006). There also are four water-supply wells located east of the dune field near the park headquarters (fig. 15). The NPS monitors these wells for nutrients, chlorine, and fecal indicator bacteria to meet Colorado drinking-water regulations. Descriptions of the water-supply wells are given in Chatman and others (1997), and water-quality records starting in 1991 are available from the NPS. Additional water-level measurements were made at 120 shallow observation wells in 1998 to study the disappearance of interdunal wetlands in GRSA (Wurstera and others, 2002).

The main water-resource issue identified by NPS is the lack of high-quality data that are needed to adequately describe the water budget for GRSA (Chatman and others, 1997). This information is needed to protect ground-water levels, surface-water levels, and streamflow on, across, and under GRSA, which is necessary for the preservation of the dunes and the adjacent wetlands. The primary threats to maintaining the balance of natural hydrologic processes are ground-water and surface-water withdrawals and long-term changes in climate. One of the main water-quality issues identified by the NPS was fecal contamination of Medano Creek in public wading areas (Chatman and others, 1997). Other issues identified include maintenance of a potable water supply and determin- ing effects of primitive camping zones and abandoned mine sites, landfills, and a firing range on water quality. Deposition of atmospheric contaminants such as nitrogen and sulfur could pose a risk to high-elevation lakes and streams in the Sangre De Cristo Mountains.

\section{Surface-Water Quality}

Water-quality samples were collected over the period 1964 to 2001 at 46 surface-water sites within the boundaries of GRSA including 40 streams, 4 springs, and 2 interdunal ponds (table 20, fig. 17). Data are available for 387 samples at these sites, the majority of which are field properties (29 percent), and major-constituent (21 percent), nutrient (9 percent), and trace-element (12 percent) analyses (table 21). Surface-water sites typically were sampled 1 to 3 times each during the period of record, although there were eight sites that were sampled 10 or more times (table 20). The agencies responsible for sample collection at these sites are summarized below. Water-quality data from 1966 to 1970 were collected by the USGS at Mosca Creek (S1) around the time that the USGS gaging station at that site was operational. Fifteen sites (S1, S4, S21-S31, S36, and S46) were sampled once each in 1976 as part of the NURE program (http://pubs.usgs.gov/ of/1997/ofr-97-0492/, accessed July 2006). Twenty sites (S1, S4, S13, S19, S20, and S32-S46) were sampled during 1990 to 1996 for the NPS through a grant from the non-profit citizens support group Friends of the Dunes. Twenty-eight samples were collected at Medano Creek (S14) from 1992 to 1996 as part of the USGS National Water Quality Assessment Program (http://water.usgs.gov/nawqa/, accessed July 2006). Two hundred fifty samples were collected by the USGS at 15 sites (S1, S3-S9, S11, S12, and S14-S18) during 1999 and 2000 to characterize baseline water-quality conditions in support of achieving Outstanding Waters Designation for several streams and springs in GRSA (Ferguson, 2003). Four sites (S2, S10, S14, and S18) were sampled during 2000 and 2001 as part of a USGS ground-water study (Rupert and Plummer, 2004).

Field properties and major-dissolved constituents for the 46 surface-water sites in GRSA are summarized in table 22. Surface water in GRSA was moderately concentrated and well buffered. Specific conductance ranged from 40 to $725 \mu \mathrm{S} / \mathrm{cm}$, and alkalinity ranged from 17 to $156 \mathrm{mg} / \mathrm{L}$. The dominant anion in surface-water samples was bicarbonate (based on $\mathrm{pH}$ and alkalinity), and the dominant cations were calcium (4.0 to $50 \mathrm{mg} / \mathrm{L}$ ), magnesium (1.1 to $9.4 \mathrm{mg} / \mathrm{L}$ ), and sodium (1.0 to 19 $\mathrm{mg} / \mathrm{L}$ ). The predominance of these constituents is attributed to weathering of silicate minerals in the Precambrian bedrock of the Sangre de Cristo Mountains. Other major anions were sulfate, which ranged from 0.6 to $28 \mathrm{mg} / \mathrm{L}$, and chloride, which ranged from 0.17 to $7.2 \mathrm{mg} / \mathrm{L}$. The relatively low chloride concentrations (median $0.79 \mathrm{mg} / \mathrm{L}$ ) indicate that atmospheric deposition is probably the main source of chloride in surface water. Although considerably less abundant than bicarbonate, sulfate concentrations were slightly higher (median $4.1 \mathrm{mg} / \mathrm{L}$ ) 
Table 20. Surface-water sampling sites in Great Sand Dunes National Park and Preserve, Colorado.

[no., number; ST, stream; SP, spring; LK, lake or pond; Cr, Creek; Colo., Colorado; GRSA, Great Sand Dunes National Monument; identification numbers beginning with GRSA from USEPA STORET all others from USGS NWIS]

\begin{tabular}{|c|c|c|c|c|c|c|}
\hline $\begin{array}{l}\text { Site no. } \\
\text { (fig. 17) }\end{array}$ & Identification no. & Station name & Latitude & Longitude & Type & Period of record \\
\hline S1 & 08234200 & Mosca Creek near Mosca, Colo. & $37^{\circ} 44^{\prime} 05^{\prime \prime}$ & $105^{\circ} 30^{\prime} 29^{\prime \prime}$ & ST & a $1966-70,76,90-93,99-2000$ \\
\hline S2 & 374303105374401 & Little Spring Creek near Source & $37^{\circ} 43^{\prime} 03^{\prime \prime}$ & $105^{\circ} 37^{\prime} 44^{\prime \prime}$ & ST & 2001 \\
\hline S3 & 374348105304001 & Morris Gulch Spring at GRSA, Colo. & $37^{\circ} 43^{\prime} 48^{\prime \prime}$ & $105^{\circ} 30^{\prime} 42^{\prime \prime}$ & SP & a $1964,99-2000$ \\
\hline S4 & 374404105302701 & Mosca Spring at GRSA, Colo. & $37^{\circ} 44^{\prime} 04^{\prime \prime}$ & $105^{\circ} 30^{\prime} 29^{\prime \prime}$ & SP & a $1964,76,91,99-2000$ \\
\hline S5 & 374416105310501 & Medano Cr below Mosca Cr at GRSA, Colo. & $37^{\circ} 44^{\prime} 16^{\prime \prime}$ & $105^{\circ} 31^{\prime} 07^{\prime \prime}$ & ST & a $1999-2000$ \\
\hline S6 & 374439105304901 & Medano $\mathrm{Cr}$ below Garden $\mathrm{Cr}$ at GRSA, Colo. & $37^{\circ} 44^{\prime} 39^{\prime \prime}$ & $105^{\circ} 30^{\prime} 51^{\prime \prime}$ & ST & a1999-2000 \\
\hline S7 & 374447105301101 & Garden Creek at Boundary at GRSA, Colo. & $37^{\circ} 44^{\prime} 47^{\prime \prime}$ & $105^{\circ} 30^{\prime} 06^{\prime \prime}$ & ST & 1999-2000 \\
\hline S8 & 374507105300201 & Buck $\mathrm{Cr}$ at Boundary at GRSA, Colo. & $37^{\circ} 45^{\prime} 07^{\prime \prime}$ & $105^{\circ} 30^{\prime} 04^{\prime \prime}$ & ST & a1999-2000 \\
\hline S9 & 374520105295801 & Sawmill Cany at Boundary GRSA, Colo. & $37^{\circ} 45^{\prime} 20^{\prime \prime}$ & $105^{\circ} 30^{\prime} 00^{\prime \prime}$ & ST & 1999 \\
\hline S10 & 374540105380201 & Big Spring Creek below Indian Spring & $37^{\circ} 45^{\prime} 40^{\prime \prime}$ & $105^{\circ} 38^{\prime} 04^{\prime \prime}$ & ST & $2000-01$ \\
\hline S11 & 374623105295901 & Castle Creek at Boundary at GRSA, Colo. & $37^{\circ} 46^{\prime} 23^{\prime \prime}$ & $105^{\circ} 30^{\prime} 01^{\prime \prime}$ & ST & 1999-2000 \\
\hline S12 & 374652105380401 & West Elk Interdunal Pond at GRSA, Colo. & $37^{\circ} 46^{\prime} 52^{\prime \prime}$ & $105^{\circ} 38^{\prime} 06^{\prime \prime}$ & LK & 1999-2000 \\
\hline $\mathrm{S} 13$ & 374745105301701 & Medano Creek at GRSA & $37^{\circ} 47^{\prime} 45^{\prime \prime}$ & $105^{\circ} 30^{\prime} 19^{\prime \prime}$ & ST & 1978,95 \\
\hline S14 & 374752105300801 & Medano Creek near Mosca, Colo. & $37^{\circ} 47^{\prime} 52^{\prime \prime}$ & $105^{\circ} 30^{\prime} 10^{\prime \prime}$ & ST & ${ }^{\mathrm{a}} 1992-96,99-2001$ \\
\hline S15 & 374823105383901 & Sand Creek at Baca Boundary at GRSA, Colo. & $37^{\circ} 48^{\prime} 23^{\prime \prime}$ & $105^{\circ} 38^{\prime} 41^{\prime \prime}$ & ST & 1999-2000 \\
\hline S16 & 374825105302601 & Little Medano Cr at Mouth at GRSA, Colo. & $37^{\circ} 48^{\prime} 25^{\prime \prime}$ & $105^{\circ} 30^{\prime} 28^{\prime \prime}$ & ST & 1999-2000 \\
\hline S17 & 374927105331101 & Cold $\mathrm{Cr}$ at Boundary at GRSA, Colo. & $37^{\circ} 49^{\prime} 27^{\prime \prime}$ & $105^{\circ} 33^{\prime} 13^{\prime \prime}$ & ST & 1999-2000 \\
\hline S18 & 374946105353301 & Sand $\mathrm{Cr}$ at North Boundary at GRSA, Colo. & $37^{\circ} 49^{\prime} 46^{\prime \prime}$ & $105^{\circ} 35^{\prime} 35^{\prime \prime}$ & ST & a1999-2001 \\
\hline S19 & GRSA_BOR_CASTLE & Castle $\mathrm{Cr}$ between Park Boundary and Dunes & $37^{\circ} 46^{\prime} 23^{\prime \prime}$ & $105^{\circ} 30^{\prime} 09^{\prime \prime}$ & ST & 1991 \\
\hline S20 & GRSA_BOR_SAND & Sand Creek at Jeep Trail Crossing & $37^{\circ} 49^{\prime} 48^{\prime \prime}$ & $105^{\circ} 35^{\prime} 26^{\prime \prime}$ & ST & 1991 \\
\hline S21 & GRSA_NURE_02 & C29077 & $37^{\circ} 44^{\prime} 03^{\prime \prime}$ & $105^{\circ} 39^{\prime} 56^{\prime \prime}$ & ST & 1976 \\
\hline S22 & GRSA_NURE_03 & C29079 & $37^{\circ} 42^{\prime} 59^{\prime \prime}$ & $105^{\circ} 38^{\prime} 03^{\prime \prime}$ & ST & 1976 \\
\hline S23 & GRSA_NURE_05 & C29083 & $37^{\circ} 41^{\prime} 52^{\prime \prime}$ & $105^{\circ} 40^{\prime} 20^{\prime \prime}$ & LK & 1976 \\
\hline S24 & GRSA_NURE_13 & C29061 & $37^{\circ} 43^{\prime} 03^{\prime \prime}$ & $105^{\circ} 30^{\prime} 04^{\prime \prime}$ & ST & 1976 \\
\hline S25 & GRSA_NURE_14 & C29469 & $37^{\circ} 43^{\prime} 50^{\prime \prime}$ & $105^{\circ} 29^{\prime} 21^{\prime \prime}$ & ST & 1976 \\
\hline S26 & GRSA_NURE_15 & C29470 & $37^{\circ} 43^{\prime} 50^{\prime \prime}$ & $105^{\circ} 28^{\prime} 02^{\prime \prime}$ & ST & 1976 \\
\hline S27 & GRSA_NURE_16 & C29679 & $37^{\circ} 53^{\prime} 21^{\prime \prime}$ & $105^{\circ} 30^{\prime} 11^{\prime \prime}$ & ST & 1976 \\
\hline S28 & GRSA_NURE_17 & C29283 & $37^{\circ} 46^{\prime} 35^{\prime \prime}$ & $105^{\circ} 30^{\prime} 30^{\prime \prime}$ & ST & 1976 \\
\hline S29 & GRSA_NURE_19 & C29654 & $37^{\circ} 49^{\prime} 37^{\prime \prime}$ & $105^{\circ} 27^{\prime} 02^{\prime \prime}$ & ST & 1976 \\
\hline $\mathrm{S} 30$ & GRSA_NURE_20 & C29655 & $37^{\circ} 51^{\prime} 10^{\prime \prime}$ & $105^{\circ} 26^{\prime} 23^{\prime \prime}$ & ST & 1976 \\
\hline S31 & GRSA_NURE_21 & C29656 & $37^{\circ} 51^{\prime} 14^{\prime \prime}$ & $105^{\circ} 26^{\prime} 22^{\prime \prime}$ & ST & 1976 \\
\hline S32 & GRSA_SLV_BEND & Medano Creek at a Large Bend & $37^{\circ} 44^{\prime} 55^{\prime \prime}$ & $105^{\circ} 30^{\prime} 47^{\prime \prime}$ & ST & 1995 \\
\hline S33 & GRSA_SLV_BOUND & Medano Creek at Park Boundary & $37^{\circ} 48^{\prime} 03^{\prime \prime}$ & $105^{\circ} 29^{\prime} 52^{\prime \prime}$ & ST & 1995 \\
\hline S34 & GRSA_SLV_BUCK & Buck Creek between Boundary and Dunes & $37^{\circ} 45^{\prime} 03^{\prime \prime}$ & $105^{\circ} 30^{\prime} 17^{\prime \prime}$ & ST & 1990-93 \\
\hline S35 & GRSA_SLV_CAMPTR & Medano Creek at Campground Trail & $37^{\circ} 44^{\prime} 43^{\prime \prime}$ & $105^{\circ} 30^{\prime} 50^{\prime \prime}$ & ST & 1995 \\
\hline S36 & GRSA_SLV_DENSP & Denton Spring & $37^{\circ} 41^{\prime} 59^{\prime \prime}$ & $105^{\circ} 31^{\prime} 26^{\prime \prime}$ & SP & $1976,91,93$ \\
\hline S37 & GRSA_SLV_DRAIN & Drainage Creek above Indian Spring & $37^{\circ} 46^{\prime} 02^{\prime \prime}$ & $105^{\circ} 37^{\prime} 30^{\prime \prime}$ & ST & 1993 \\
\hline S38 & GRSA_SLV_FLUME & Medano Creek at Flume & $37^{\circ} 47^{\prime} 43^{\prime \prime}$ & $105^{\circ} 30^{\prime} 21^{\prime \prime}$ & ST & 1995 \\
\hline S39 & GRSA_SLV_INDSP & Indian Spring & $37^{\circ} 46^{\prime} 00^{\prime \prime}$ & $105^{\circ} 37^{\prime} 31^{\prime \prime}$ & SP & $1990,91,93$ \\
\hline$S 40$ & GRSA_SLV_LIMED & Little Medano $\mathrm{Cr}$ and Medano Cr Confluence & $37^{\circ} 47^{\prime} 37^{\prime \prime}$ & $105^{\circ} 30^{\prime} 34^{\prime \prime}$ & ST & 1995 \\
\hline S41 & GRSA_SLV_LITMED & Little Medano Creek & $37^{\circ} 48^{\prime} 23^{\prime \prime}$ & $105^{\circ} 30^{\prime} 33^{\prime \prime}$ & ST & 1990-91 \\
\hline S42 & GRSA_SLV_MEDCAS & Medano Creek at Castle Creek & $37^{\circ} 46^{\prime} 20^{\prime \prime}$ & $105^{\circ} 30^{\prime} 25^{\prime \prime}$ & ST & $1990,92,93$ \\
\hline S43 & GRSA_SLV_NBOARD & Medano Creek at North Boardwalk & $37^{\circ} 44^{\prime} 23^{\prime \prime}$ & $105^{\circ} 31^{\prime} 04^{\prime \prime}$ & ST & 1995 \\
\hline S44 & GRSA_SLV_SANDPI & Medano Creek at Sand Pit & $37^{\circ} 45^{\prime} 49^{\prime \prime}$ & $105^{\circ} 30^{\prime} 22^{\prime \prime}$ & ST & 1995 \\
\hline S45 & GRSA_SLV_SBOARD & Medano Creek at South Boardwalk & $37^{\circ} 44^{\prime} 19^{\prime \prime}$ & $105^{\circ} 31^{\prime} 10^{\prime \prime}$ & ST & 1991,95 \\
\hline S46 & GRSA_SLV_SHOCKY & Medano Creek at Shockey's Crossing & $37^{\circ} 47^{\prime} 42^{\prime \prime}$ & $105^{\circ} 30^{\prime} 26^{\prime \prime}$ & ST & $1976,90-91,95$ \\
\hline
\end{tabular}

${ }^{\text {aSampled }} 10$ or more times during period of record. 


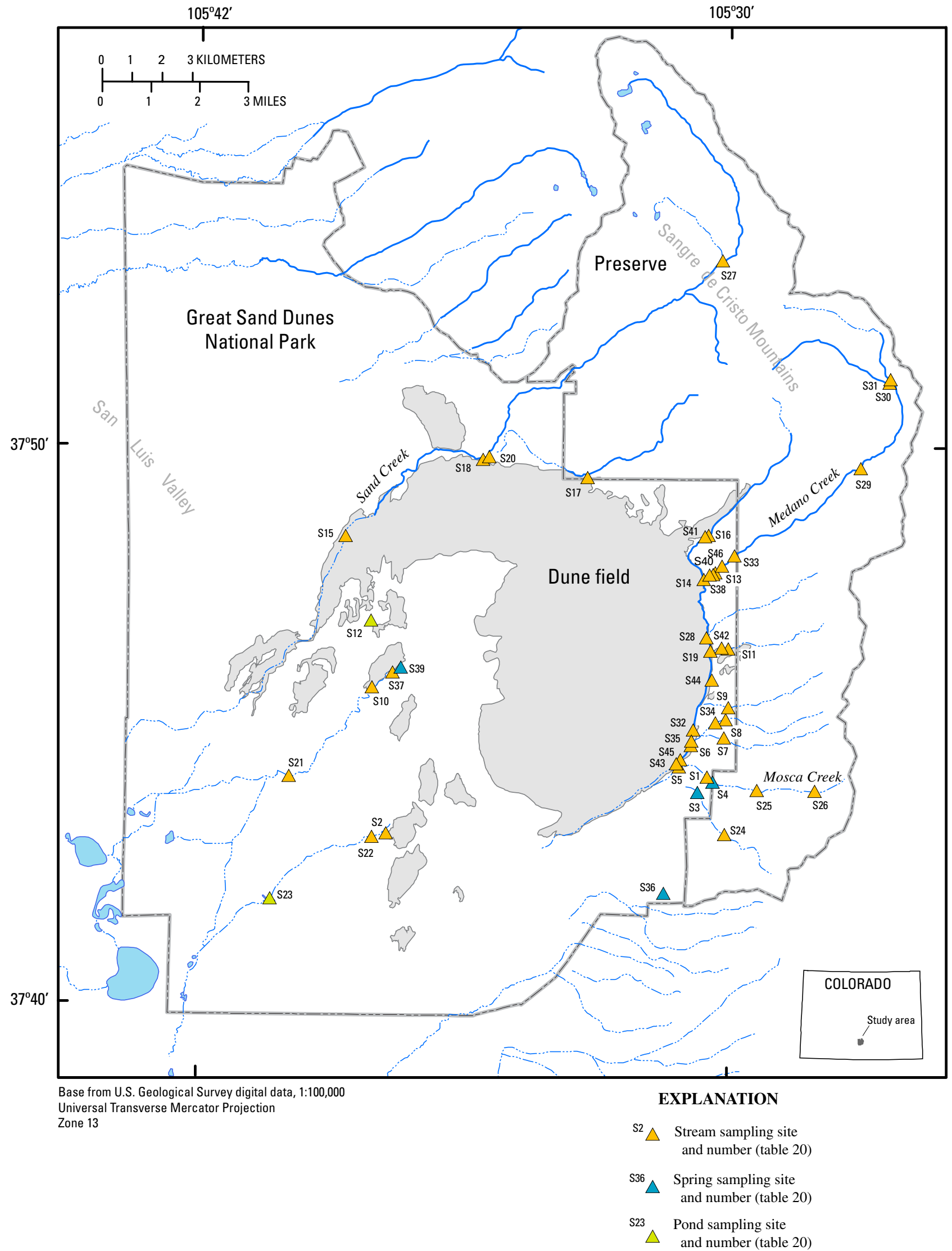

Figure 17. Location of surface-water sampling sites in Great Sand Dunes National Park and Preserve, Colorado. 


\begin{tabular}{|c|c|c|c|c|c|c|c|c|}
\hline $200 Z$ & i & i & i & i & i & 1 & i & \\
\hline 1002 & $\nabla$ & $\forall$ & i & $\nabla$ & i & $i$ & i & $\checkmark$ \\
\hline 0002 & $\stackrel{2}{1}$ & $m$ & $m$ & $m$ & i & g) & i & $\simeq$ \\
\hline $666 \mathrm{l}$ & $\stackrel{2}{2}$ & $\vec{\nabla}$ & $\bar{\nabla}$ & $\bar{\forall}$ & i & ㅍ & $i$ & 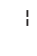 \\
\hline $866 \mathrm{l}$ & i & i & i & i & i & i & i & i \\
\hline L66l & i & i & i & i & i & i & i & i \\
\hline $966 \mathrm{l}$ & $N$ & $N$ & $N$ & $N$ & i & $i$ & - & I \\
\hline S66l & 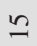 & $\simeq$ & $=$ & $=$ & - & లి & $\nabla$ & i \\
\hline t66l & $m$ & $m$ & $\simeq$ & 工 & i & 1 & i & I \\
\hline E66l & $n$ & 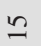 & \pm & $n$ & 0 & 1 & $\mathrm{i}$ & i \\
\hline Z66l & $\forall$ & $\forall$ & - & $\forall$ & - & 1 & - & 1 \\
\hline I66l & 으 & & $ㅇ$ & 으 & $i$ & 1 & i & I \\
\hline 066l & 1 & 6 & $i$ & 6 & $i$ & $i$ & i & i \\
\hline $686 \mathrm{l}$ & $i$ & i & i & 1 & $i$ & 1 & i & i \\
\hline $886 \mathrm{l}$ & $i$ & i & i & $i$ & $\mathrm{i}$ & i & $\mathrm{i}$ & i \\
\hline$\angle 86 \mathrm{~L}$ & 1 & 1 & 1 & 1 & $i$ & 1 & i & i \\
\hline 986l & i & i & 1 & 1 & i & i & i & I \\
\hline S86L & $i$ & 1 & $i$ & $i$ & $i$ & 1 & i & ' \\
\hline 七86l & 1 & i & $i$ & $i$ & $i$ & i & i & i \\
\hline E86l & $i$ & 1 & i & $i$ & $i$ & 1 & i & I \\
\hline $286 \mathrm{~L}$ & $i$ & 1 & $\mathrm{i}$ & $i$ & $i$ & 1 & i & I \\
\hline $186 \mathrm{l}$ & $i$ & i & $i$ & $i$ & $i$ & 1 & ; & I \\
\hline 086l & i & 1 & 1 & $i$ & $\mathrm{i}$ & i & i & i \\
\hline $6 \angle 6 l$ & $i$ & $i$ & i & 1 & i & $i$ & i & I \\
\hline $8 \angle 6 l$ & - & - & - & - & - & i & i & I \\
\hline$\angle L 6 I$ & 1 & I & $i$ & 1 & $i$ & 1 & i & i \\
\hline $9 \angle 6 \mathrm{l}$ & $n$ & i & $i$ & 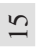 & 1 & 1 & i & I \\
\hline SL6l & i & i & $i$ & $i$ & i & i & i & I \\
\hline$t \angle 6 l$ & i & 1 & 1 & $i$ & $i$ & 1 & i & I \\
\hline EL6L & 1 & i & 1 & 1 & 1 & $i$ & i & ! \\
\hline ZL6l & 1 & i & 1 & $i$ & $i$ & $i$ & i & ! \\
\hline lLGl & 1 & i & $i$ & $i$ & $i$ & i & i & i \\
\hline $0<6 l$ & \pm & i & $i$ & $i$ & $i$ & $i$ & i & i \\
\hline 696l & $m$ & $i$ & i & $i$ & $i$ & 1 & i & ! \\
\hline $896 \mathrm{l}$ & 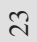 & $n$ & $n$ & $i$ & $i$ & 1 & i & I \\
\hline L96l & $n$ & - & - & $i$ & $i$ & $i$ & i & I \\
\hline 996l & - & I & i & $i$ & $i$ & 1 & i & ! \\
\hline S96l & ! & $i$ & $i$ & $i$ & $\mathrm{i}$ & i & i & i \\
\hline \multirow[t]{2}{*}{ †96l } & $N$ & $N$ & $N$ & $N$ & i & 1 & $i$ & I \\
\hline & 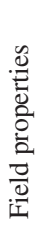 & 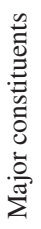 & 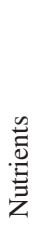 & 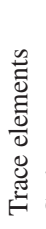 & 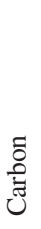 & 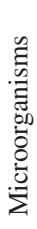 & $\begin{array}{l}\frac{\mathscr{d}}{0} \\
.0 \\
0 \\
0 \\
0\end{array}$ & $\stackrel{0}{2}$ \\
\hline
\end{tabular}

than chloride indicating geologic as well as atmospheric sources of sulfate.

Spatial variation in selected water-quality constituents at eight surface-water sites in GRSA are shown in figure 18. Sites S18, S16, and S14 are located on larger perennial streams that originate at high elevations (above 3,600 m) in the Sangre de Cristo Mountains. Sites S8 and S1 are on smaller streams on the east side of the park that originate at lower elevations (below 3,000 m) and are intermittent in their upper reaches. Sites S3 and S4 are springs on the east side of the park and site S12 is a shallow interdunal pond on the sand sheet. The median specific conductance at the eight sites ranged from 68 to $279 \mu \mathrm{S} / \mathrm{cm}$ and was lowest for the perennial streams. This reflects greater contributions of dilute snowmelt and surface runoff from high-elevation areas compared to the springs, which are dominated by ground-water discharge. The two intermittent streams (S8 and S1) were similar to the springs indicating they are likely fed by similar ground-water sources. The ratio of major dissolved constituents was similar among all the sites with the exception of the interdunal pond (S12), which had a lower calcium to alkalinity ratio perhaps reflecting a different ground-water source. This explanation is reasonable considering site $\mathrm{S} 12$ is the only site located downgradient from the dune field. Median $\mathrm{pH}$ at the eight sites ranged from 7.4 to 8.1 and was not notably different among the eight sites except for site S12, which had a wider range of values than the other sites. Median dissolved oxygen concentrations ranged from 7.4 to $13.4 \mathrm{mg} / \mathrm{L}$ and, similar to $\mathrm{pH}$, were elevated at site $\mathrm{S} 12$ relative to other sites. Because site S12 is a shallow pond, these differences could result from photosynthesis by aquatic vegetation, which produces oxygen and consumes carbon dioxide causing increases in dissolved oxygen concentrations and $\mathrm{pH}$ in surface water during the day.

Seasonal variations in selected major-dissolved constituents at Medano Creek (S14) are shown in figure 19. Concentrations were highest during low-flow conditions in winter and then decrease by more than a factor of two in May and June during peak snowmelt. Similar seasonal patterns were observed at other stream (S1, S8, and S18) and spring (S3 and S4) sites although dilution during snowmelt was less pronounced in springs reflecting a greater contribution of ground water. Medano Creek (S14) was the only surface-water site that had sufficient data to test for changes in water quality with time but only for field properties. No statistically significant trends were detected for specific conductance $(\mathrm{p}=0.581), \mathrm{pH}$ $(\mathrm{p}=0.576)$, or dissolved oxygen $(\mathrm{p}=0.311)$ at this site during 1992 to 2000.

Nutrients measured in surface-water samples from GRSA include ammonia, nitrate, nitrite, organic nitrogen, orthophosphate, and total phosphorus (table 22). Inorganic nitrogen species were measured at 16 surface-water sites, whereas phosphorus species were measured only at 3 sites. Nitrogen compounds in all samples were low, and most had concentrations below laboratory reporting levels. For example, 34 percent of samples had ammonia concentrations below laboratory reporting levels, 74 percent for nitrate, and 96 percent for 
Table 22. Summary of selected water-quality data for surface-water sites in Great Sand Dunes National Park and Preserve, Colorado, 1964 to 2001.

[No. number; chronic aquatic-life (and water-supply) standards from Colorado Department of Public Health and Environment (2003); <, less than; $>$, greater than; --, not reported; bd, below detection; ${ }^{\circ} \mathrm{C}$, degrees Celsius; $\mathrm{mg} / \mathrm{L}$, milligrams per liter; $\mu \mathrm{S} / \mathrm{cm}$, microsiemens per centimeter at 25 ${ }^{\circ} \mathrm{C} ; \mathrm{CaCO}_{3}$, calcium carbonate; $\mathrm{N}$, nitrogen; $\mathrm{P}$, phosphorous; col/100 mL, colonies per 100 milliliters; $\mu \mathrm{g} / \mathrm{L}$, micrograms per liter; some nutrients and trace elements have multiple reporting limits]

\begin{tabular}{|c|c|c|c|c|c|c|}
\hline Constituent or property & No. sites & $\begin{array}{c}\text { No. analyses } \\
\text { (no. censored) }\end{array}$ & $\begin{array}{l}\text { Minimum } \\
\text { value }\end{array}$ & $\begin{array}{c}\text { Median } \\
\text { value }\end{array}$ & $\begin{array}{c}\text { Maximum } \\
\text { value }\end{array}$ & $\begin{array}{c}\text { Aquatic-life } \\
\text { (water-supply) } \\
\text { standard }\end{array}$ \\
\hline \multicolumn{7}{|c|}{ Field properties } \\
\hline Temperature, water $\left({ }^{\circ} \mathrm{C}\right)$ & 29 & 312 & 0.0 & 10.0 & 31.5 & $<20$ \\
\hline Oxygen, dissolved (mg/L) & 16 & 228 & 5.7 & 8.0 & 18.9 & $>6.0$ \\
\hline $\mathrm{pH}$ (standard units) & 37 & 280 & 6.4 & 7.8 & 9.5 & $6.5-9.0(5.0-9.0)$ \\
\hline Specific conductance $(\mu \mathrm{S} / \mathrm{cm})$ & 37 & 273 & 40 & 125 & 725 & -- \\
\hline \multicolumn{7}{|c|}{ Major constituents } \\
\hline Alkalinity $\left(\mathrm{mg} / \mathrm{L}\right.$ as $\left.\mathrm{CaCO}_{3}\right)$ & 15 & 110 & 17 & 45 & 156 & -- \\
\hline Calcium, dissolved (mg/L) & 27 & 140 & 4.0 & 12 & 50 & -- \\
\hline Chloride, dissolved (mg/L) & 12 & 74 & .17 & .79 & 7.2 & $\left({ }^{a} 250\right)$ \\
\hline Fluoride, dissolved (mg/L) & 12 & 50 & .1 & .2 & 1.0 & -- \\
\hline Magnesium, dissolved (mg/L) & 27 & 140 & 1.1 & 3.4 & 9.4 & -- \\
\hline Potassium, dissolved (mg/L) & 17 & 60 & .40 & .74 & 16 & -- \\
\hline Silica, dissolved (mg/L) & 12 & 56 & 5.6 & 12 & 42 & -- \\
\hline Sodium, dissolved (mg/L) & 17 & 66 & 1.0 & 2.9 & 19 & -- \\
\hline Sulfate, dissolved (mg/L) & 23 & 126 & .6 & 4.1 & 28 & $(250)$ \\
\hline \multicolumn{7}{|c|}{ Nutrients, carbon, and microorganisms } \\
\hline Ammonia, dissolved (mg/L as $\mathrm{N})$ & 16 & $109(37)$ & $<0.002$ & 0.005 & 0.192 & 0.02 \\
\hline Nitrate, dissolved (mg/L as N) & 16 & $108(80)$ & ${ }^{\mathrm{b}} .013$ & $<.05$ & .13 & (10) \\
\hline Nitrite, dissolved (mg/L as N) & 16 & $109(105)$ & $<.01$ & $<.01$ & .01 & $.05(1.0)$ \\
\hline Organic nitrogen, dissolved (mg/L as $\mathrm{N})$ & 2 & $34(32)$ & $<.20$ & $<.20$ & .42 & -- \\
\hline Orthophosphate, dissolved (mg/L as $\mathrm{P}$ ) & 3 & $40(25)$ & $<.01$ & $<.01$ & .02 & -- \\
\hline Phosphorus, dissolved (mg/L as P) & 2 & $35(22)$ & $<.01$ & $<.01$ & .03 & -- \\
\hline Organic carbon, dissolved (mg/L) & 2 & 5 & 1.9 & 2.8 & 5.6 & -- \\
\hline Fecal coliform (col/100 mL) & 9 & $166(31)$ & $<1$ & 4.5 & 2,500 & '200 \\
\hline Fecal streptococci (col/100 mL) & 9 & $166(1)$ & $<1$ & 138 & 17,000 & -- \\
\hline \multicolumn{7}{|c|}{ Trace elements } \\
\hline Arsenic, dissolved $(\mu \mathrm{g} / \mathrm{L})$ & 13 & $19(12)$ & bd & 0.0 & 22 & d50 (10) \\
\hline Cadmium, dissolved $(\mu \mathrm{g} / \mathrm{L})$ & 25 & $90(81)$ & bd & $<.5$ & $<2.5$ & ${ }^{\mathrm{e}} 0.2(5)$ \\
\hline Copper, dissolved ( $\mu \mathrm{g} / \mathrm{L})$ & 25 & $93(53)$ & bd & $<1.3$ & 95 & e $4(1,300)$ \\
\hline Iron, dissolved $(\mu \mathrm{g} / \mathrm{L})$ & 16 & $58(11)$ & bd & 84 & 270 & ${ }^{\mathrm{d}} 1,000\left({ }^{\mathrm{a}} 300\right)$ \\
\hline Lead, dissolved ( $\mu \mathrm{g} / \mathrm{L})$ & 25 & $93(91)$ & bd & $<1.0$ & 23 & ${ }^{\mathrm{e}} 1(50)$ \\
\hline Manganese, dissolved ( $\mu \mathrm{g} / \mathrm{L})$ & 27 & $130(24)$ & bd & 7.8 & 386 & $50\left({ }^{a} 50\right)$ \\
\hline Mercury, dissolved ( $\mu \mathrm{g} / \mathrm{L})$ & 12 & $12(12)$ & bd & bd & $<.20$ & -- \\
\hline Nickel, dissolved ( $\mu \mathrm{g} / \mathrm{L})$ & 11 & $11(11)$ & bd & .0 & $<5.0$ & e25 (100) \\
\hline Selenium, dissolved ( $\mu \mathrm{g} / \mathrm{L})$ & 25 & $93(81)$ & bd & $<1.0$ & 20 & $4.6(50)$ \\
\hline Silver, dissolved ( $\mu \mathrm{g} / \mathrm{L})$ & 24 & $88(88)$ & bd & $<.2$ & $<4.0$ & ${ }^{\mathrm{e}} 0.08\left({ }^{\mathrm{a}} 100\right)$ \\
\hline Uranium, dissolved $(\mu \mathrm{g} / \mathrm{L})$ & 17 & $17(2)$ & 0.42 & $\stackrel{\mathrm{b}}{\cdot 4}$ & 11 & e592 (30) \\
\hline Zinc, dissolved $(\mu \mathrm{g} / \mathrm{L})$ & 24 & $90(56)$ & bd & $<10$ & 44 & ${ }^{\mathrm{e}} 61\left({ }^{\mathrm{a}} 5,000\right)$ \\
\hline
\end{tabular}

aSecondary standard based on aesthetic properties such as taste, odor, and staining.

${ }^{\mathrm{b}}$ Minimum reported value less than minimum censored value.

'Primary contact recreation.

${ }^{\mathrm{d}}$ Total recoverable concentration.

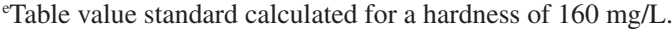



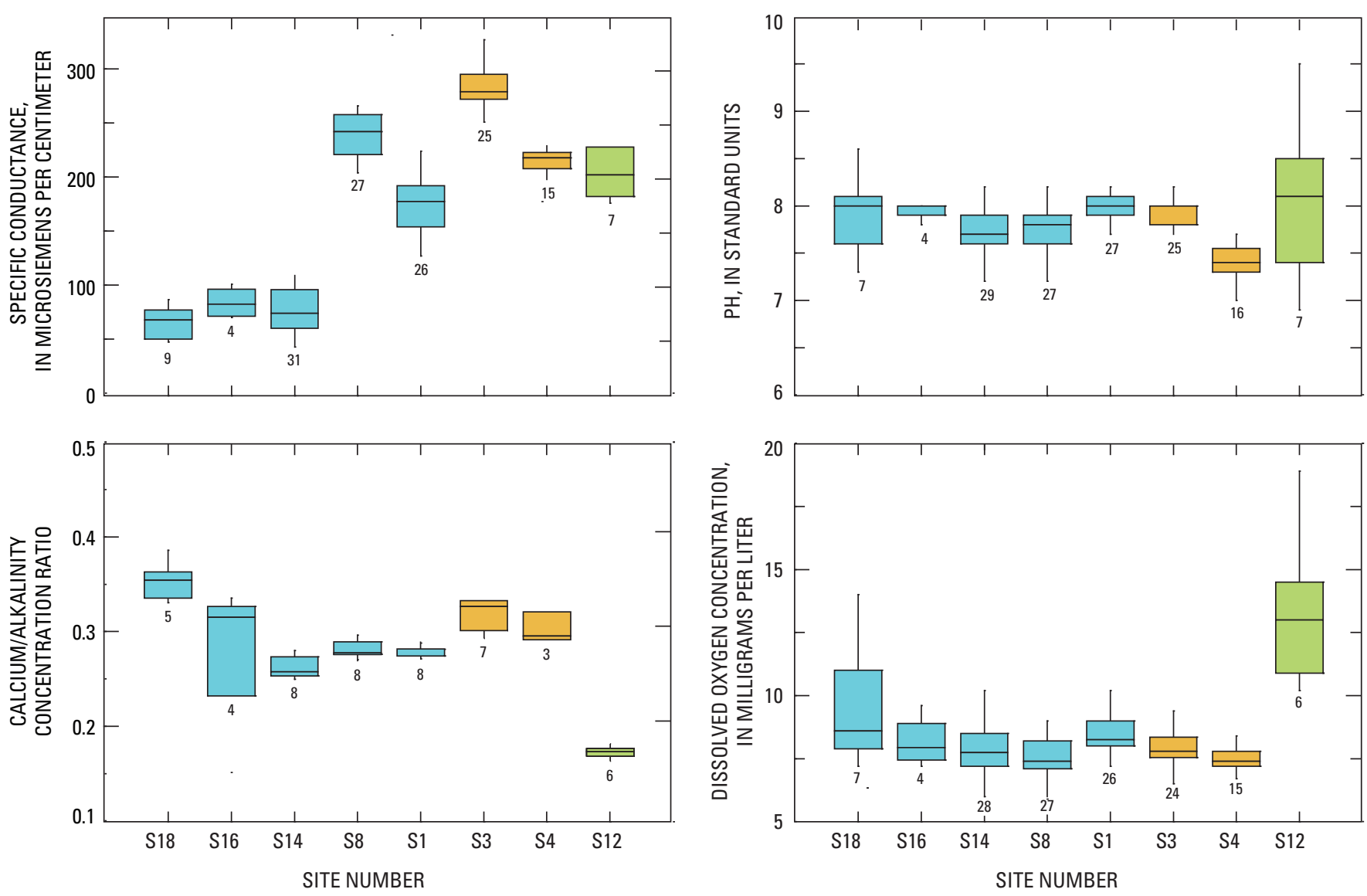

EXPLANATION

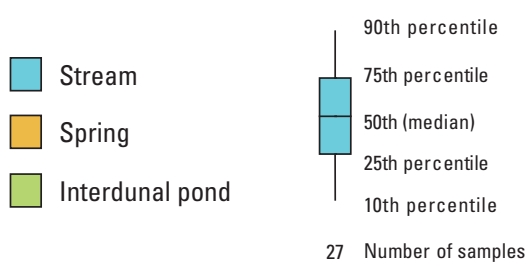

Figure 18. Spatial variation in water quality at selected sites in Great Sand Dunes National Park and Preserve, Colorado.

nitrite. Detected ammonia concentrations were less than 0.05 $\mathrm{mg} / \mathrm{L}$ except for one sample collected at a shallow interdunal pond (S12), which had a concentration of $0.192 \mathrm{mg} / \mathrm{L}$. Detected concentrations of nitrate ranged from 0.013 to 0.13 $\mathrm{mg} / \mathrm{L}$ and were elevated during winter months $(0.07$ to 0.13 $\mathrm{mg} / \mathrm{L})$ relative to summer months $(0.02$ to $0.07 \mathrm{mg} / \mathrm{L})$ likely because of greater ground-water inputs combined with lower biological demand. The major source of inorganic nitrogen to streams in GRSA is atmospheric deposition (Burns, 2004), which originates from fossil fuel combustion and agriculture. Low nitrate concentrations at the stream sites, which are all at lower elevations of the park, indicate most atmospherically deposited nitrogen is retained by soils and vegetation. A recent study of several high-elevation lakes in GRSA, however, showed elevated nitrate concentrations (up to $0.3 \mathrm{mg} / \mathrm{L}$ ) during the growing season (Nanus, 2005). These results may indicate that nitrogen deposition is exceeding biological uptake and that high-elevation areas in the park may be in the early stages of nitrogen saturation (Burns, 2004). Orthophosphate and total phosphorous concentrations also were low with 72 percent of samples below the reporting levels, and all detected concentrations less than or equal to $0.03 \mathrm{mg} / \mathrm{L}$.

Dissolved concentrations of 12 trace elements for sites with four or more samples are summarized in table 22. Most concentrations were less than laboratory reporting levels and only iron (78 percent) and manganese (33 percent) had a substantial number of detected concentrations above $10 \mu \mathrm{g} / \mathrm{L}$. Detected iron concentrations ranged from 7 to $270 \mu \mathrm{g} / \mathrm{L}$, and were highest in Medano Creek (51 to $250 \mu \mathrm{g} / \mathrm{L}$ ). High dissolved iron in Medano Creek was somewhat unexpected given 

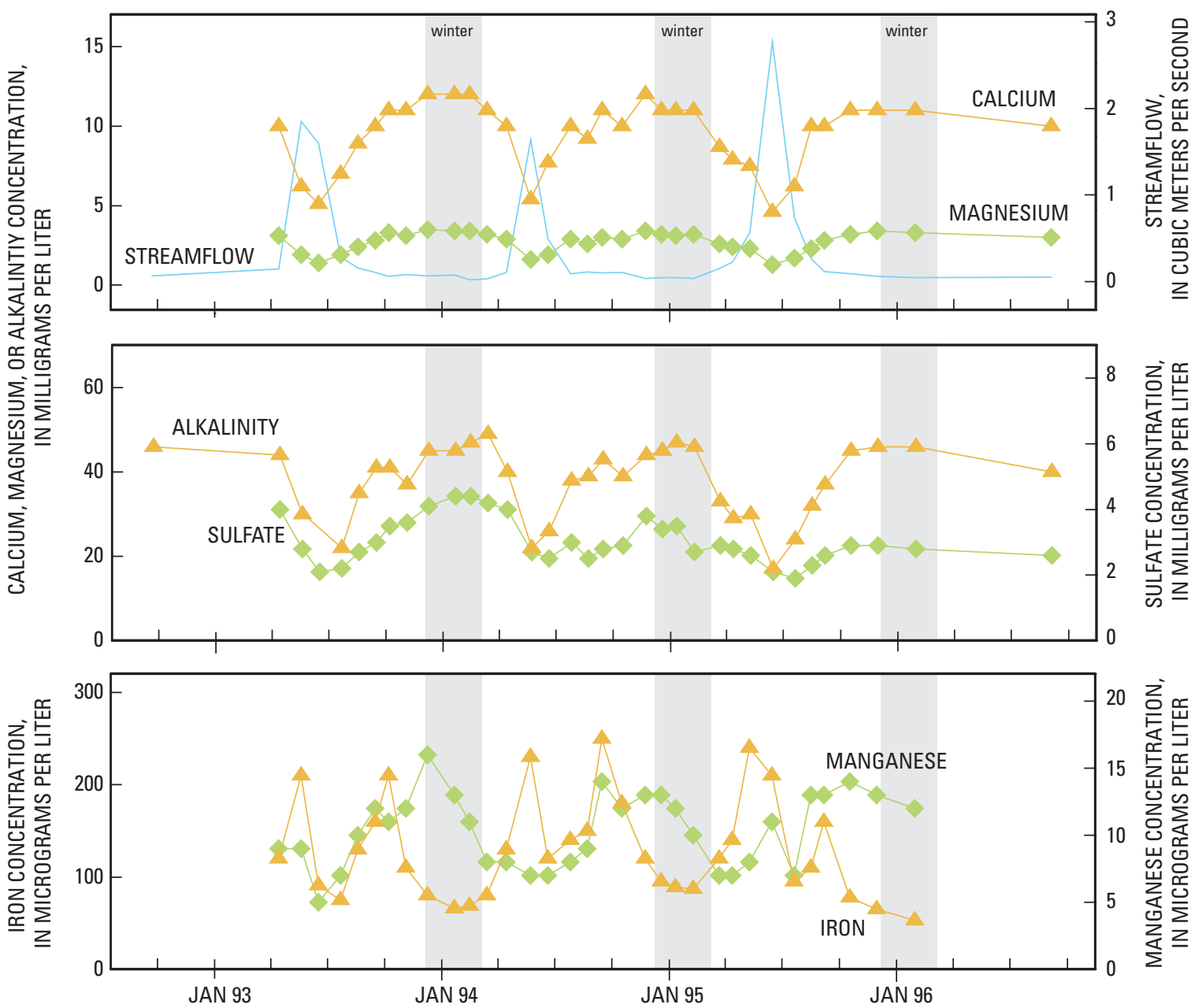

Figure 19. Seasonal variation in selected constituent concentrations at Medano Creek near Mosca (S14) in Great Sand Dunes National Park and Preserve, Colorado.

that stream $\mathrm{pH}$ is neutral, which indicates that some colloidal iron is not removed by sample filtration. Detected manganese concentrations ranged from 1.8 to $386 \mu \mathrm{g} / \mathrm{L}$ and were highest in Buck Creek ( 7 to $386 \mu \mathrm{g} / \mathrm{L}$ ). The source of manganese may be mineralized quartz veins that occur throughout the Sangre de Cristo Mountains. These veins were prospected for gold, lead, and zinc in the late 1880s and there are several prospect pits located in the southern part of the park (Chatman and others, 1997). Seasonal patterns for iron and manganese at Medano Creek (S14) are shown in figure 19. Iron showed a strong seasonal pattern with concentrations peaking during snowmelt and again in early fall. Higher concentrations during runoff could be related to increased colloidal iron in the stream during snowmelt or flushing of organically-complexed iron from soils. The reason for the peak in iron concentrations during fall is unclear. In contrast to iron, manganese concentrations were lowest during snowmelt and peaked during baseflow conditions in winter, indicating manganese is related to inputs of ground water to streamflow.
Other constituents measured in surface-water samples collected in GRSA include dissolved organic carbon (1.9 to $5.6 \mathrm{mg} / \mathrm{L}$ ), fecal coliform (less than 1 to $2,500 \mathrm{col} / 100 \mathrm{~mL}$ ) and fecal streptococci (less than 1 to $17,000 \mathrm{col} / 100 \mathrm{~mL}$ ). Fecal coliform and fecal streptococci are considered indicator bacteria for the presence of fecal contamination because they are present in large numbers in the feces and intestinal tracts of humans and other warm-blooded animals. Fecal coliform and total coliform were measured seasonally at nine sites in GRSA during 1999 and 2000. The highest concentrations of fecal coliform were measured in Medano Creek (S5), which had a median concentration of $420 \mathrm{col} / 100 \mathrm{~mL}$. The higher concentrations relative to other sampling sites in the park may be related to visitor use as most park facilities are located in the vicinity of Medano Creek. In addition, wading in the lower reaches of Medano Creek is a popular activity of park visitors during the summer months. Fecal streptococci were substantially higher in Morris Gulch Spring (S3) and Mosca Spring (S4) than the other seven sites, and indicated a strong seasonal 
pattern with peak concentrations in June and July $(1,100$ to $17,000 \mathrm{col} / 100 \mathrm{~mL}$ ). Despite elevated fecal streptococci counts, the fecal coliform counts at these two sites were low (less than 1 to $66 \mathrm{col} / 100 \mathrm{~mL}$ ). Fecal streptococci are sometimes used as an indicator of nonhuman fecal contamination, the increased concentrations may be caused by wildlife that use the springs as a source of drinking water.

Comparison to Water-Quality Standards. Water-quality use classifications and standards for all stream segments in Colorado are established by the Colorado Water Quality Control Commission (Colorado Department of Public Health and Environment, 2003). Standards for the Closed Basin-San Luis Valley (segment 10), which includes Sand Creek and Medano Creek and all tributaries and wetlands, from the source to the mouth, are listed in table 22 . None of the reaches within this segment were listed in 2006 as impaired for water quality (http://www.cdphe.state.co.us/op/wqcc/SpecialTopics/303(d)/ 303dtmdlpro.html, accessed July 2006). The designated uses for this segment are cold-water aquatic life class 1, recreation 1a, agriculture, and water supply. Streams designated for cold-water aquatic life class 1 (the most sensitive use) should have temperatures less than $20^{\circ} \mathrm{C}$, dissolved oxygen greater than $6.0 \mathrm{mg} / \mathrm{L}$ (7.0 $\mathrm{mg} / \mathrm{L}$ during spawning), and $\mathrm{pH}$ values ranging from 6.5 to 9.0. For surface-water samples in GRSA, 296 of 312 temperature measurements were below the $20{ }^{\circ} \mathrm{C}$ standard, 223 of 228 dissolved oxygen measurements were above the $6.0 \mathrm{mg} / \mathrm{L}$ standard, and 277 of $280 \mathrm{pH}$ values were within the 6.5 to 9.0 range. Most of the sites that exceeded the $20{ }^{\circ} \mathrm{C}$ temperature standard are on the edge of the dune field where temperatures on the sand surface can reach $60{ }^{\circ} \mathrm{C}$ during hot summer days (Chatman and others, 1997). The six dissolved oxygen measurements below $6.0 \mathrm{mg} / \mathrm{L}$ were made in summer at sites along the edge of the dune field where water temperatures commonly exceed $20^{\circ} \mathrm{C}$. The three $\mathrm{pH}$ values that were outside the standard range were measured at Denton Spring (S36; pH 6.4), Indian Spring (S39; pH 9.1), and West Elk interdunal pond (S12; pH 9.5). The instream standard for fecal coliform is $200 \mathrm{col} / 100 \mathrm{~mL}$ for recreation class $1 \mathrm{a}$, which was exceeded in 10 of 166 measurements. Most of the exceedances were samples collected from Medano Creek during the summer months (June-August). All park facilities, campgrounds, and picnic grounds are located along the lower reach of Medano Creek. Ammonia was the only nutrient species that exceeded the aquatic-life standard $(0.02 \mathrm{mg} / \mathrm{L})$. The highest concentration $(0.192 \mathrm{mg} / \mathrm{L})$ was measured at a shallow interdunal pond (S12). Exceedances in other samples ranged from 0.02 to $0.05 \mathrm{mg} / \mathrm{L}$. Of the trace elements there were a few exceedances for copper (3 of 93), lead (1 of 93), selenium ( 5 of 93), and manganese (11 of 130). The manganese exceedances were limited to three sites: Buck Creek (S8), a shallow interdunal pond (S12), and Indian Spring (S39). As mentioned previously, elevated manganese, particularly in Buck Creek, may be related to mineralized veins in the Sangre de Cristo Mountains. Elevated manganese at the interdunal pond and Indian Spring site is more likely caused by reductive dissolution of aquifer materials as both of these sites are fed by ground water discharging downgradient from the dune field.

GRSA is pursuing "Outstanding Natural Waters" designation for several surface-water segments in the park, which would provide the highest level of regulatory protection provided under the Clean Water Act and Colorado regulations. To quality for this designation, water bodies must have exceptional recreational or ecological significance unmodified by human activities, and existing water quality must meet specified standards for 12 properties and constituents $(\mathrm{pH}$, dissolved oxygen, E. coli bacteria, nitrate, ammonia, cadmium, copper, lead, manganese, selenium, silver, and zinc) (Colorado Department of Public Health and Environment, 2006). The regulations state that the 85 th percentile of existing water-quality data shall not exceed State criteria for ammonia, nitrate, and dissolved metals, the 50th percentile for total recoverable metals, the 15 th percentile for dissolved oxygen, the geometric mean for E. coli, and the range between the 15th and 85 th percentiles for $\mathrm{pH}$. Data collected by the USGS in 1999 and 2000 to describe baseline conditions indicate that surface water in the park meets water-quality criteria for determining Outstanding Waters Designation (Ferguson, 2003).

\section{Ground-Water Quality}

Water-quality results for 20 samples collected at 20 ground-water wells (table 23, fig. 15) within the boundaries of GRSA between 1946 and 2000 are summarized in table 24. Sites G3 and G10 were sampled for specific conductance in 1946; sites G1, G2, and G6 were sampled for major constituents in 1968; and G4 and G5 were sampled for major constituents and nutrients in 1980. The most complete data set is for 13 wells (G8-G9, G11-G20) sampled by the USGS in 2000 as part of a study to determine ground-water flow direction, water quality, recharge sources, and age in GRSA. These samples were analyzed for major constituents, organic carbon, chlorofluorocarbons, and isotopes of hydrogen, oxygen, and carbon. The results of this ground-water study, which are summarized below, are published in Rupert and Plummer (2004). The water quality of the unconfined aquifer was dominated by calcium, sodium, and bicarbonate. Specific conductance ranged from 69 to $333 \mu \mathrm{S} / \mathrm{cm}$, and alkalinity ranged from 27 to $125 \mathrm{mg} / \mathrm{L}$. As ground water traveled under the dune field through the unconfined aquifer, the percentage of calcium decreased and the percentage of sodium increased because of cation exchange with clays. Water quality of the confined aquifer was similar, except that magnesium and dissolved oxygen were depleted relative to concentrations in the unconfined aquifer. Age dating of the ground water indicates it takes more than 60 years for water in the unconfined aquifer to flow from the mountain front to the western edge of the dune field. Ground water in the deeper confined aquifer was estimated to be approximately 30,000 years old. Insufficient data exist to quantify the hydrologic connection between the unconfined and confined aquifers. Available data for ground-water wells are insufficient to describe seasonal patterns or temporal trends in water quality because 
Table 23. Ground-water sampling sites in Great Sand Dunes National Park and Preserve, Colorado.

[no., number; well depth in meters; identification numbers and station names from USGS NWIS]

\begin{tabular}{|c|c|c|c|c|c|c|}
\hline $\begin{array}{l}\text { Site no. } \\
\text { (fig. 15) }\end{array}$ & Identification no. & Station name & Latitude & Longitude & Well depth & $\begin{array}{l}\text { Period of } \\
\text { record }\end{array}$ \\
\hline G1 & 373915105414501 & NA04001232CCC1 & $37^{\circ} 39^{\prime} 48^{\prime \prime}$ & $105^{\circ} 41^{\prime} 27^{\prime \prime}$ & 17.1 & 1968 \\
\hline $\mathrm{G} 2$ & 373915105414502 & NA04001232CCC2 & $37^{\circ} 39^{\prime} 48^{\prime \prime}$ & $105^{\circ} 41^{\prime} 27^{\prime \prime}$ & 3.4 & 1968 \\
\hline G3 & 373953105412301 & NA04001121CC & $37^{\circ} 39^{\prime} 52^{\prime \prime}$ & $105^{\circ} 41^{\prime} 25^{\prime \prime}$ & 14.6 & 1946 \\
\hline G4 & 374012105410401 & NA04001232BAA & $37^{\circ} 40^{\prime} 12^{\prime \prime}$ & $105^{\circ} 41^{\prime} 06^{\prime \prime}$ & 9.1 & 1980 \\
\hline G5 & 374040105392001 & NA04001233AAA1 & $37^{\circ} 40^{\prime} 40^{\prime \prime}$ & $105^{\circ} 39^{\prime} 21^{\prime \prime}$ & 11.9 & 1980 \\
\hline G6 & 374345105321001 & SC02707334DD & $37^{\circ} 43^{\prime} 45^{\prime \prime}$ & $105^{\circ} 32^{\prime} 12^{\prime \prime}$ & 107 & 1968 \\
\hline G7 & 374407105360401 & SC04001212AAA & $37^{\circ} 44^{\prime} 07^{\prime \prime}$ & $105^{\circ} 36^{\prime} 06^{\prime \prime}$ & 3.1 & 2000 \\
\hline G8 & 374410105312701 & SC02707303ABB & $37^{\circ} 44^{\prime} 09^{\prime \prime}$ & $105^{\circ} 31^{\prime} 28^{\prime \prime}$ & 4.0 & 2000 \\
\hline G9 & 374414105310201 & SC02607334DDC & $37^{\circ} 44^{\prime} 13^{\prime \prime}$ & $105^{\circ} 31^{\prime} 04^{\prime \prime}$ & 107 & 2000 \\
\hline G10 & 374431105431101 & NA04001101CA & $37^{\circ} 44^{\prime} 30^{\prime \prime}$ & $105^{\circ} 43^{\prime} 13^{\prime \prime}$ & 15.6 & 1946 \\
\hline G11 & 374557105372901 & SC04101235ABB2 & $37^{\circ} 45^{\prime} 56^{\prime \prime}$ & $105^{\circ} 37^{\prime} 31^{\prime \prime}$ & 107 & 2000 \\
\hline G12 & 374558105372801 & SC04101235ABB1 & $37^{\circ} 45^{\prime} 57^{\prime \prime}$ & $105^{\circ} 37^{\prime} 30^{\prime \prime}$ & 23.0 & 2000 \\
\hline G13 & 374605105372301 & SC04101226DCD & $37^{\circ} 46^{\prime} 05^{\prime \prime}$ & $105^{\circ} 37^{\prime} 24^{\prime \prime}$ & 4.0 & 2000 \\
\hline G14 & 374655105365901 & SC04101223DDD & $37^{\circ} 46^{\prime} 54^{\prime \prime}$ & $105^{\circ} 37^{\prime} 01^{\prime \prime}$ & 8.0 & 2000 \\
\hline G15 & 374657105375401 & SC04101223CCD1 & $37^{\circ} 46^{\prime} 56^{\prime \prime}$ & $105^{\circ} 37^{\prime} 55^{\prime \prime}$ & 23.0 & 2000 \\
\hline G16 & 374657105375402 & SC04101223CCD2 & $37^{\circ} 46^{\prime} 56^{\prime \prime}$ & $105^{\circ} 37^{\prime} 55^{\prime \prime}$ & 3.5 & 2000 \\
\hline G17 & 374746105385201 & SC04101215CDC & $37^{\circ} 47^{\prime} 45^{\prime \prime}$ & $105^{\circ} 38^{\prime} 53^{\prime \prime}$ & 32.0 & 2000 \\
\hline G18 & 374746105385202 & SC04101215CDC1 & $37^{\circ} 47^{\prime} 45^{\prime \prime}$ & $105^{\circ} 38^{\prime} 53^{\prime \prime}$ & 9.5 & 2000 \\
\hline G19 & 374746105385203 & SC04101215CDC2 & $37^{\circ} 47^{\prime} 45^{\prime \prime}$ & $105^{\circ} 38^{\prime} 53^{\prime \prime}$ & 120 & 2000 \\
\hline G20 & 374954105355101 & SC02501236ADD & $37^{\circ} 49^{\prime} 54^{\prime \prime}$ & $105^{\circ} 35^{\prime} 53^{\prime \prime}$ & 12.0 & 2000 \\
\hline
\end{tabular}

none of the wells in GRSA have been sampled more than once during the period of record. The water quality of the 20 wells was compared to water-supply standards established by the CDPHE (Colorado Department of Public Health and Environment, 2006), although none of these wells are used for water supply. For the reported constituents, the $\mathrm{pH}$ of one sample was just above the water supply standard and one sample (G13) exceeded the secondary drinking-water standards for iron and manganese. Elevated iron and manganese concentrations in ground water can be caused by reductive dissolution of oxide minerals present in the aquifer sediments when the dissolved oxygen concentration becomes depleted.

\section{Summary and Suggestions for Vital Signs Monitoring}

Review of historical records identified 387 water-quality samples collected at 46 surface-water sites in GRSA from 1964 to 2001 . The majority of results for these 387 samples were field properties (29 percent), and major-constituent (21 percent), nutrient ( 9 percent), and trace-element (12 percent) analyses. Surface-water sites typically were sampled 1 to 3 times each during the period of record, although there were eight sites that were sampled 10 or more times. Streamflowgaging stations have been operated at the two primary perennial streams, Medano Creek and Sand Creek, since 1992. Surface water is a calcium-bicarbonate type and dissolved solids primarily are derived from weathering of crystalline bedrock in the Sangre De Cristo Mountains. Visitor use and park facilities likely have a minimal effect on water quality, except perhaps in the lower reaches of Medano Creek. Preliminary data indicate that lakes at high elevations in the park may be in early stages of nitrogen saturation because of atmospheric nitrogen deposition. Sufficient data were available to test for temporal trends in water quality only for field properties at one site, which showed no trends. The designated uses for this segment are cold-water aquatic life class 1, recreation 1a, agriculture, and water supply. Available water-quality data indicate most surface-water sites meet Colorado water-quality standards for Outstanding Natural Waters designation, which the NPS is pursuing for streams in GRSA. Twenty water-quality samples have been collected at 20 ground-water wells in the park from 1946 to 2000. Most of these samples were collected in 2000 by the USGS as part of a study to determine ground-water flow direction, water quality, recharge sources, and age in GRSA. 
Table 24. Summary of selected water-quality data for ground-water sites in Great Sand Dunes National Park and Preserve, 1946 to 2000.

[no., number; water-supply standards published by Colorado Department of Public Health and Environment (2006); <, less than; --, not reported; ${ }^{\circ} \mathrm{C}$, degrees Celsius; $\mathrm{mg} / \mathrm{L}$, milligrams per liter; $\mu \mathrm{S} / \mathrm{cm}$, microsiemens per centimeter at $25^{\circ} \mathrm{C} ; \mathrm{CaCO}_{3}$, calcium carbonate; $\mathrm{N}$, nitrogen; P, phosphorous; $\mu \mathrm{g} / \mathrm{L}$, micrograms per liter]

\begin{tabular}{|c|c|c|c|c|c|c|}
\hline Constituent or property & No. sites & $\begin{array}{l}\text { No. analyses } \\
\text { (no. censored) }\end{array}$ & $\begin{array}{l}\text { Minimum } \\
\text { value }\end{array}$ & $\begin{array}{c}\text { Median } \\
\text { value }\end{array}$ & $\begin{array}{l}\text { Maximum } \\
\text { value }\end{array}$ & $\begin{array}{c}\text { Water-supply } \\
\text { standard }\end{array}$ \\
\hline \multicolumn{7}{|c|}{ Field properties } \\
\hline Temperature, water $\left({ }^{\circ} \mathrm{C}\right)$ & 6 & 6 & 9.5 & 11.7 & 14.5 & -- \\
\hline Oxygen, dissolved (mg/L) & 13 & 13 & 0.2 & 4.1 & 7.4 & -- \\
\hline $\mathrm{pH}$ (standard units) & 17 & 17 & 6.5 & 7.7 & 9.1 & $5.0-9.0$ \\
\hline Specific conductance $(\mu \mathrm{S} / \mathrm{cm})$ & 20 & 20 & 68 & 156 & 499 & -- \\
\hline \multicolumn{7}{|c|}{ Major constituents } \\
\hline Alkalinity $\left(\mathrm{mg} / \mathrm{L}\right.$ as $\left.\mathrm{CaCO}_{3}\right)$ & 17 & 17 & 28 & 64 & 213 & -- \\
\hline Calcium, dissolved (mg/L) & 17 & 17 & 2.4 & 15 & 40 & -- \\
\hline Chloride, dissolved (mg/L) & 17 & 17 & .44 & 1.3 & 11 & a 250 \\
\hline Fluoride, dissolved (mg/L) & 17 & 17 & .00 & .43 & 1.4 & 2.0 \\
\hline Magnesium, dissolved (mg/L) & 17 & 17 & .39 & 2.7 & 8.6 & -- \\
\hline Potassium, dissolved (mg/L) & 17 & 17 & .70 & 4.6 & 18 & -- \\
\hline Silica, dissolved (mg/L) & 17 & 17 & 13 & 40 & 69 & -- \\
\hline Sodium, dissolved (mg/L) & 17 & 17 & 1.9 & 11 & 84 & -- \\
\hline Sulfate, dissolved (mg/L) & 17 & 17 & .44 & 5.3 & 53 & a250 \\
\hline \multicolumn{7}{|c|}{ Other constituents } \\
\hline Nitrate, dissolved (mg/L as N) & 2 & 2 & 0.09 & -- & 0.40 & 10 \\
\hline Nitrogen, total (mg/L as $\mathrm{N})$ & 2 & 2 & .46 & -- & .74 & -- \\
\hline Phosphorus, total (mg/L as P) & 2 & 2 & .05 & -- & .13 & -- \\
\hline Carbon, organic (mg/L) & 13 & $13(3)$ & $<.15$ & 0.45 & 5.2 & -- \\
\hline Iron, dissolved ( $\mu \mathrm{g} / \mathrm{L})$ & 15 & $15(10)$ & $<10$ & $<10$ & 3,365 & а300 \\
\hline Manganese, dissolved ( $\mu \mathrm{g} / \mathrm{L})$ & 15 & $15(7)$ & b 1.8 & $<3.2$ & 116 & a50 \\
\hline
\end{tabular}

Water-quality issues for the park include effects of visitor use and atmospheric deposition of contaminants, maintenance of potable water supplies, and attainment of Outstanding Natural Waters designation. The primary threats to water resources in the park are surface- and ground-water withdrawals and long-term climate changes. Based on a review of the historical data, the following suggestions are provided for consideration in designing a water-quality monitoring plan for GRSA.

- Establish a long-term water-quality monitoring station or "sentinel site" on Medano Creek at the streamflowgaging station. Monitor for core field properties, major constituents, and nutrients during different hydrologic conditions.
- Monitor the lower reaches of Medano Creek for bacterial indicators during the summer tourist season.

- Periodically conduct water-quality surveys of highelevation lakes and streams in the park to monitor the effects of deposition of atmospheric contanimants.

- Additional surface-water sites, including ground-water springs, could be sampled periodically to address special issues or to achieve a better understanding of the factors controlling water quality.

- Maintain the existing network of streamflow-gaging stations and ground-water wells for monitoring the effects of human stressors on water resources. 


\section{Little Bighorn Battlefield National Monument}

\section{Environmental Setting}

The Little Big Horn Battlefield National Monument (LIBI) is located within the Little Bighorn River Valley in south-central Montana near the town of Crow Agency (fig. 20). The monument covers $3.08 \mathrm{~km}^{2}$, ranges in elevation from 933 to $1,050 \mathrm{~m}$, and sits on terraces above the floodplain of the Little Bighorn River. Only a small area (approximately 0.20 $\mathrm{km}^{2}$ ) along the western boundary of the monument extends into the floodplain of the river. The landscape is characterized by rolling hills covered by grasslands (74 percent) and shrublands (11 percent) interspersed with pockets of coniferous trees (10 percent) (Britten and others, 2006). The dominant grasses are bluebunch wheatgrass and blue grama-needlegrass, and the main shrubs are hawthorn, chokeberry, silver sage, and big sagebrush. Climate is typical of colder short-grass prairies. Mean monthly air temperatures at Crow Agency range from $-6.8{ }^{\circ} \mathrm{C}$ in January to $22.2^{\circ} \mathrm{C}$ in July (http://www.wrcc.dri.edu/ summary/climsmmt.html, accessed June 2006). Average annual precipitation is $39 \mathrm{~cm}$; May $(6.1 \mathrm{~cm})$ and June $(6.0 \mathrm{~cm})$ are the wettest months. The area receives about $105 \mathrm{~cm}$ of snowfall each year between October and April.

LIBI is underlain by Upper Cretaceous sedimentary rocks of the Judith River Formation and Bearpaw Shale. The Judith River Formation is fine-grained sandstone interbedded with shales and forms an important aquifer in the area (Tuck, 2003). The Bearpaw Shale is a dark grey fossiliferous shale that is nearly impermeable with respect to ground water. Along the floodplain, the Cretaceous sediments are covered by Quaternary alluvium, which is composed of unconsolidated gravel, sand, silt, and clay. The alluvium, which typically is less than $9 \mathrm{~m}$ thick, forms the principal aquifer in the Little Bighorn River Basin (Tuck, 2003).

Land-use activities in LIBI are related to visitor use (nearly 400,000 visitors per year), which peaks in July and August (Britten and others, 2006). The monument has a visitor center, several administrative buildings and residences, numerous cultural features, three trails, and $15 \mathrm{~km}$ of roads. A shallow alluvial well adjacent to the Little Bighorn River is the primary water supply for the monument. The water supply is tested regularly for fecal indicators, nitrogen species, and organic compounds according to water-supply regulations mandated by the MTDEQ; results of the tests are available at http://www.deq.state.mt.us/wqinfo/pws/reports.asp (accessed June 2006). Wastewater for the park is handled by a septic system. Land surrounding the monument is within the Crow Indian Reservation. The primary land use in the reservation is irrigated agriculture along the valley floor for cultivation of alfalfa, pasture grass, corn, and sugar beets (Tuck, 2003). The higher terraces and foothill areas primarily are used as rangeland for cattle. Effects of these agricultural activities on nutrient, bacteria, and sediment are the main water-quality issues for the Little Bighorn River.

\section{Water Resources}

LIBI is situated along the lower reaches of the Little Bighorn River (fig. 20), which drains an area of about 3,370 $\mathrm{km}^{2}$. The river originates in the Bighorn Mountains in Wyoming, and drains north for about $130 \mathrm{~km}$ through foothills and a broad alluvial valley. Lodge Grass and Pass Creeks are the main perennial tributaries, and Owl and Reno Creeks are the largest ephemeral tributaries. LIBI is bounded on the west by the Little Bighorn River, but there are no perennial or ephemeral streams flowing through the monument. The Little Bighorn River is an important source of water for irrigation in the valley, and as a water supply for LIBI and the town of Crow Agency. The USGS has operated as many as 17 streamflow-gaging stations in the Little Bighorn River Basin (http:// mt.water.usgs.gov/pub/MTStations.pdf, accessed June 2006); however, only 4 of these stations were active in 2006. The NPS operates a streamflow-gaging station on the Little Bighorn River (station 453400107263001) along the western edge of the monument (fig. 20). This gage, which started operation in November 1999, is maintained by the NPS as part of a Federal water-rights agreement. The range of daily streamflow values at station 453400107263001 from 1999-2005 is shown by month in figure 21 . The streamflow pattern shows that discharge peaks in May and June during snowmelt. Intense rainstorms and saturated or frozen soils are additional factors contributing to increased runoff during the spring. Streamflow decreases rapidly through July and is lowest in August and September because of low precipitation rates and high evapotranspiration rates. During summer months, water also is diverted from the river for irrigation.

An excellent description of ground-water hydrology in the Little Bighorn River Basin is presented by Tuck (2003) from which the following discussion is summarized. Quaternary alluvium and the Cretaceous Judith River Formation compose the two main aquifers in the basin and the primary water source for domestic and stock supplies. The alluvial aquifer, which is limited to areas adjacent to the river and major tributaries, is composed of unconsolidated alluvial deposits that have moderate to low permeability. Recharge to the alluvium is by infiltration of precipitation primarily during fall before the ground freezes and in early spring when evapotranspiration is minimal. Other sources of recharge include inflow from the underlying Judith River Formation, canal leakage, excess irrigation water, bank storage, and inflow from ephemeral tributaries. The direction of ground-water flow generally is from the valley margins, where it is recharged, towards the Little Bighorn River, where it discharges into the river contributing as much as 18 percent of the annual daily mean streamflow. The fine-grained sediment comprosing the Judith River aquifer results in lower permeability than the overlying alluvial aquifer. Recharge to the Judith River aquifer primarily is by infiltration of precipitation with additional inputs from infiltration of streamflow across outcrops, canal leakage, and bank storage. The direction of ground-water 


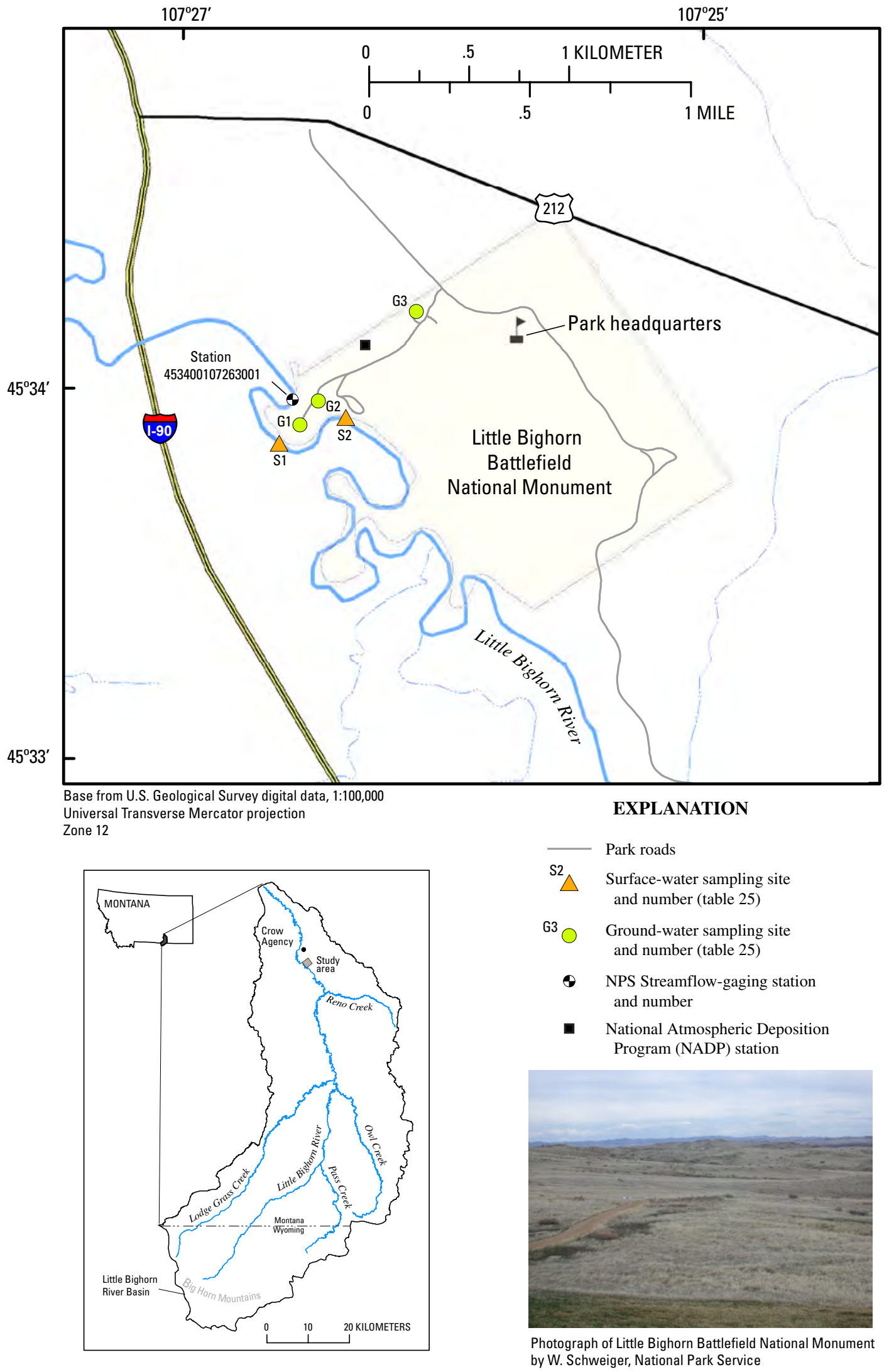

Figure 20. Location of water-quality sampling sites near Little Bighorn Battlefield National Monument, Montana. 


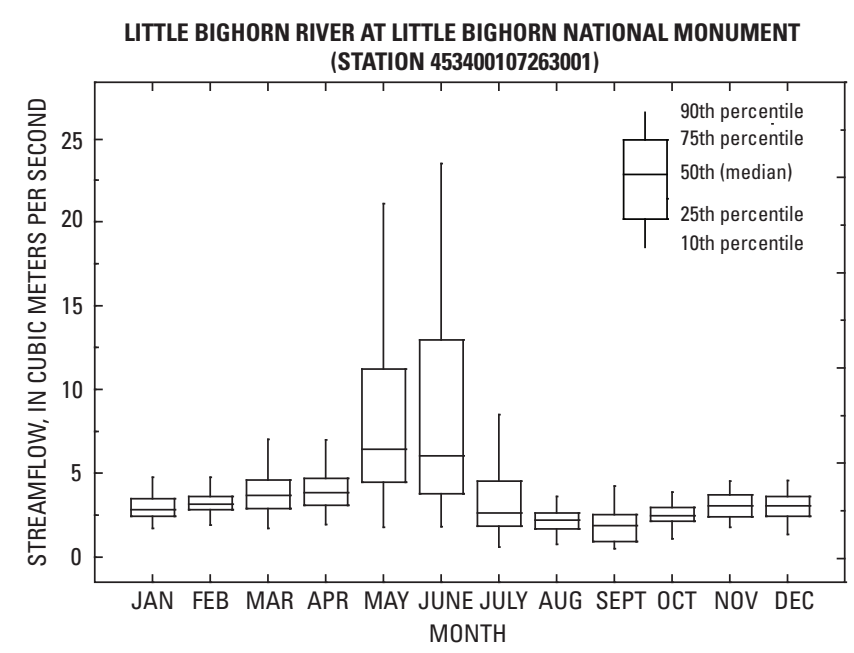

Figure 21. Daily streamflow range at Little Bighorn River (station 453400107263001) in Little Bighorn National Battlefield Monument, Montana, 1999-2005.

flow is from outcrop areas west of the river towards the Little Bighorn River, where it discharges to the alluvial aquifer and directly to the river.

Water levels in 32 ground-water wells in the basin were measured monthly to bimonthly by the USGS during 1994 to 1995 to document seasonal changes in water levels (Tuck, 2003). Water-level data for these wells are available from the USGS NWIS web interface at http://nwis.waterdata.usgs.gov/ usa/nwis/gwlevels (accessed June 2006). Annual water-level measurements were made by USGS at one well in LIBI (G3, fig. 20) from 1977 to 1999 as part of a state-wide groundwater monitoring network. The hydrograph for this well shows that ground-water levels were highest in the early part of the record because of above average precipitation during most of the 1970s (fig. 22).

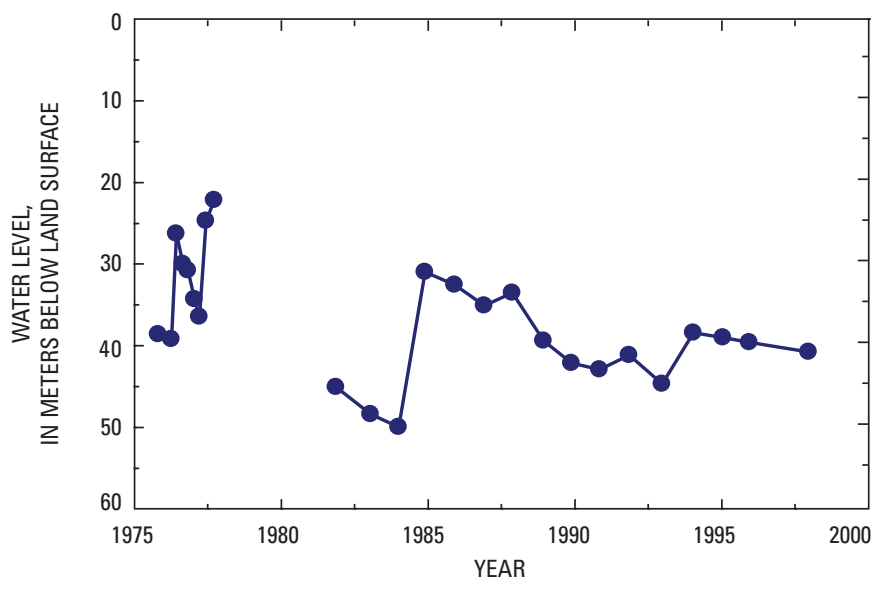

Figure 22. Ground-water level measurements at site G3 in Little Bighorn National Battlefield Monument, Montana.
The park also has a NADP station (fig. 20), which has been operated jointly by the USGS and NPS since 1984 . The NADP is a national network of precipitation monitoring sites that measures weekly precipitation chemistry and precipitation amount; data for the network are available at http://nadp.sws. uiuc.edu/ (accessed June 2006). The site at LIBI was established to represent atmospheric deposition in shortgrass prairie ecosystems.

\section{Surface-Water Quality}

LIBI is bounded on the west by the Little Bighorn River, but there are no perennial or ephemeral streams originating within the park. The only surface-water stations in LIBI are sites S1 and S2 both of which are on the Little Bighorn River adjacent to the west boundary of the monument (fig. 20). There were four water-quality samples collected at site S1 during 1999 and 2000 that were analyzed for field properties, major constituents, nutrients, trace elements, and suspended sediment. These samples were collected by the USGS for the NPS and analyzed at the USGS National Water Quality Laboratory (http://nwql.usgs.gov/, accessed June 2006). Site S2 was sampled in 1977 by the USGS and analyzed at the Montana Bureau of Mines and Geology analytical laboratory (http://www.mbmg.mtech.edu/analytical.htm, accessed June 2006).

Water-quality data for the two sampling sites on the Little Bighorn River adjacent to the monument are summarized in table 26. Surface water was fairly concentrated and well buffered; specific conductance ranged from 489 to $786 \mu \mathrm{S} / \mathrm{cm}$, and alkalinity ranged from 155 to $241 \mathrm{mg} / \mathrm{L}$. Calcium was the dominant cation, and bicarbonate (based on alkalinity and $\mathrm{pH}$ ) and sulfate were the dominant anions. These dissolved constituents are likely derived from weathering of carbonate minerals and gypsum in the marine sedimentary rocks that are common in the area (Vuke and others, 2000). Of the five samples, major-constituent concentrations generally were highest on March 22, 2000, which probably reflects a greater contribution of ground water to the river during low-flow conditions before spring runoff. Nutrient concentrations were low and close to laboratory reporting levels in all five samples except for the sample collected on May 19, 2000. Because this sample was collected during increased streamflow conditions $\left(1,970 \mathrm{ft}^{3} / \mathrm{s}\right)$, the elevated nitrogen concentrations may be caused by runoff from agricultural areas upstream from the sampling site. The high total phosphorus concentration $(1.97 \mathrm{mg} / \mathrm{L})$ in this sample probably is related to the elevated suspended-sediment concentration $(2,662 \mathrm{mg} / \mathrm{L})$ because erosion can transport considerable amounts of suspended phosphorus to surface waters (Mueller and others, 1995). Dissolved trace-element concentrations were less than $20 \mu \mathrm{g} / \mathrm{L}$ in all five samples. Low trace-element concentrations reflect neutral stream-water $\mathrm{pH}$ values (range 7.7 to 8.5 ) and the lack of urban and mining areas upstream from the sampling sites. Neither surface-water site had a sufficient period of record to test for changes in water quality with time. 
Table 25. Water-quality sampling sites near Little Bighorn Battlefield National Monument, Montana.

[no., number; Well depth in meters; --, no data; ST, stream; GW, ground water; AL, alluvial; JR; Judith River; identification numbers and station names from NWIS]

\begin{tabular}{cclccccc}
\hline $\begin{array}{c}\text { Site no. } \\
\text { (fig. 20) }\end{array}$ & Identification number & \multicolumn{1}{c}{ Station name } & Latitude & Longitude & Type & $\begin{array}{c}\text { Well } \\
\text { depth }\end{array}$ & Aquifer \\
\hline S1 & 453350107263301 & Little Bighorn River near Bighorn Battlefield & $45^{\circ} 33^{\prime} 50^{\prime \prime}$ & $17^{\circ} 26^{\prime} 33^{\prime \prime}$ & ST & -- & -- \\
S2 & 453355107261801 & Little Bighorn River & $45^{\circ} 33^{\prime} 55^{\prime \prime}$ & $17^{\circ} 26^{\prime} 18^{\prime \prime}$ & ST & -- & -- \\
G1 & 453354107262801 & 03S35E18DCCB01 & $45^{\circ} 33^{\prime} 54^{\prime \prime}$ & $107^{\circ} 26^{\prime} 28^{\prime \prime}$ & GW & 3 & AL \\
G2 & 453358107262401 & 03S35E18DCBD01 & $45^{\circ} 33^{\prime} 58^{\prime \prime}$ & $107^{\circ} 26^{\prime} 24^{\prime \prime}$ & GW & 37 & JR \\
G3 & 453413107260201 & 03S35E18DABD01 & $45^{\circ} 34^{\prime} 13^{\prime \prime}$ & $107^{\circ} 26^{\prime} 02^{\prime \prime}$ & GW & 122 & JR \\
\hline
\end{tabular}

Comparison to Water-Quality Standards. Water-use classifications and water-quality standards for all stream segments in Montana are established by the MTDEQ (http://www.deq. state.mt.us/wqinfo/Standards/Index.asp, accessed June 2006). The Little Bighorn River and tributaries below Grass Creek are classified as B-2, which is surface water suitable for domestic water supply, recreation, aquatic life (salmonid fishes), and agricultural and industrial water supply. No stream reaches in this segment are on the most recent (2006) 303(d) list for the State of Montana (http://www.deq.state.mt.us/wqinfo/, accessed June 2006). Aquatic-life and human-health standards established by the State of Montana for surface waters are listed in table 26. The sample collected on May 19, 2000, had constituent concentrations that exceeded trace-element standards for total cadmium, copper, iron, and lead. Concentrations of total trace elements were elevated in this sample likely because it was collected during a period of elevated sediment transport.

\section{Ground-Water Quality}

Water-quality data are available for six samples collected at three ground-water wells in LIBI (fig. 20) that were analyzed for field properties, major constituents, nutrients, trace elements, and pesticides. Site G1 is a shallow well located in the alluvial aquifer in the floodplain of the Little Bighorn River. Sites G2 and G3 are deeper wells that penetrate into the Cretaceous Judith River Formation. All three wells were sampled in 1977 by the USGS as part of a state-wide groundwater study conducted by the Montana Bureau of Mines and Geology (http://www.mbmg.mtech.edu/, accessed June 2006). The USGS also sampled site G1 in 1999 and 2000 and site G2 in 1945.

The water-quality results for samples collected at groundwater wells within the boundaries of LIBI are summarized in table 27. All of the samples had elevated concentrations of dissolved constituents with dissolved solids greater than 800 $\mathrm{mg} / \mathrm{L}$. Sodium was the dominant cation, and alkalinity and sulfate were the dominant anions. Elevated dissolved solids result from weathering of salts and soluble minerals in marine shales that underlie the drainage basin (Vuke and others, 2000). Comparison of major constituents in ground water at the three well sites indicates that dissolved solid concentrations increase with well depth, primarily because of increasing sodium and sulfate concentrations (fig. 23). A possible explanation for the observed change in ground-water quality is that calcium, released from dissolution of gypsum, is progressively exchanged for sodium on clays in the marine shale as ground water moves downgradient through the aquifer.

Despite elevated dissolved solids, concentrations of other constituents in the six ground-water samples generally were quite low. Nutrient concentrations were low in all the groundwater samples (table 27). Nitrate concentrations were well below the $2 \mathrm{mg} / \mathrm{L}$ background level defined for ground water by Mueller and Helsel (1996). These low nutrient concentrations indicate that rangeland and agricultural lands adjacent to the monument did not appear to be affecting nutrient levels in the LIBI wells at the time of sampling. Of the eight trace elements measured in ground water, only iron, lithium, and manganese were detected at concentrations above $10 \mu \mathrm{g} / \mathrm{L}$. Elevated iron and manganese concentrations in ground water can be caused by reductive dissolution of oxide minerals present in aquifer materials. Lithium concentration tended to increase with well depth similar to sodium, indicating that lithium may be released by cation exchange with clays in the marine shales. Concentrations of the pesticides and organochlorine compounds measured at site G1 on May 18, 2000, were all below the laboratory reporting levels for these compounds. None of the wells in LIBI had a sufficient period of record to test for changes in ground-water quality with time.

Comparison to Water-Quality Standards. Although wells in LIBI are not used for water supply, they were compared to human-health standards established by the MTDEQ (http:// www.deq.state.mt.us/wqinfo/Standards/Index.asp, accessed June 2006). None of the wells had constituent concentrations that exceeded the human-health standards, although four samples exceeded the secondary manganese standard of 50 $\mu \mathrm{g} / \mathrm{L}$, and three exceeded the secondary iron standard of 300 $\mu \mathrm{g} / \mathrm{L}$. Secondary standards are based on aesthetic properties such as taste, odor, and staining. Elevated iron and manganese concentrations can affect the taste of drinking water and cause staining and scaling of plumbing systems. All of the wells also exceeded the USEPA secondary drinking-water standard for sulfate $(250 \mathrm{mg} / \mathrm{L})$ and dissolved solids $(500 \mathrm{mg} / \mathrm{L})$. 
Table 26. Summary of selected water-quality data for the Little Bighorn River adjacent to Little Bighorn Battlefield National Monument, Montana.

[chronic aquatic-life and human-health standards from www.deq.state.mt.us/wqinfo/Standards/Index.asp; <, less than; >, greater than; --, not reported; ft $3 / \mathrm{s}$, cubic feet per second; ${ }^{\circ} \mathrm{C}$, degrees Celsius; mg/L, milligrams per liter; $\mu \mathrm{S} / \mathrm{cm}$, microsiemens per centimeter at $25{ }^{\circ} \mathrm{C}$; $\mathrm{CaCO}_{3}$, calcium carbonate; $\mathrm{N}$, nitrogen; $\mathrm{P}$, phosphorous; $\mu \mathrm{g} / \mathrm{L}$, micrograms per liter]

\begin{tabular}{|c|c|c|c|c|c|c|}
\hline \multirow[b]{2}{*}{ Constituent or property } & \multicolumn{4}{|c|}{ S1 (fig. 20) } & \multirow{2}{*}{$\begin{array}{c}\text { S2 (fig. 20) } \\
6 / 28 / 1977\end{array}$} & \multirow{2}{*}{$\begin{array}{c}\text { Aquatic-life } \\
\text { (human-health) } \\
\text { standards }\end{array}$} \\
\hline & 7/21/1999 & 9/1/1999 & $3 / 22 / 2000$ & $5 / 19 / 2000$ & & \\
\hline Discharge, instantaneous $\left(\mathrm{ft}^{3} / \mathrm{s}\right)$ & 148 & 109 & 144 & 1,970 & -- & -- \\
\hline Temperature, water $\left({ }^{\circ} \mathrm{C}\right)$ & 22 & 15.5 & 3.5 & 13.5 & 28 & -- \\
\hline Oxygen, dissolved (mg/L) & 8.2 & 8.5 & 11.8 & 7.7 & -- & $>4.0$ \\
\hline Specific conductance $(\mu \mathrm{S} / \mathrm{cm})$ & 489 & 542 & 786 & 568 & 519 & -- \\
\hline Sediment, suspended (mg/L) & 28 & 47 & 11 & 2,662 & -- & -- \\
\hline \multicolumn{7}{|c|}{ Major constituents } \\
\hline Alkalinity $\left(\mathrm{mg} / \mathrm{L}\right.$ as $\left.\mathrm{CaCO}_{3}\right)$ & 179 & 184 & 241 & 155 & 180 & -- \\
\hline Calcium, dissolved (mg/L) & 47 & 57 & 65 & 48 & 54 & -- \\
\hline Silica, dissolved (mg/L) & 5.9 & 5.3 & 4.6 & 7.6 & 5.5 & -- \\
\hline Sodium, dissolved $(\mathrm{mg} / \mathrm{L})$ & 19 & 24 & 45 & 36 & 24 & -- \\
\hline Sulfate, dissolved (mg/L) & 83 & 117 & 183 & 129 & 99 & (b250) \\
\hline \multicolumn{7}{|c|}{ Nutrients } \\
\hline Ammonia, dissolved (mg/L as $\mathrm{N}$ ) & $<0.02$ & 0.032 & $<0.02$ & 0.116 & -- & ${ }^{\mathrm{c}} 0.9$ \\
\hline Nitrate, dissolved (mg/L as $\mathrm{N}$ ) & $<.05$ & $<.05$ & $<.05$ & .148 & 0.023 & (10) \\
\hline Nitrite, dissolved (mg/L as N) & $<.01$ & $<.01$ & $<.01$ & .023 & -- & $(1.0)$ \\
\hline Organic nitrogen, dissolved ( $\mathrm{mg} / \mathrm{L}$ as $\mathrm{N})$ & -- & .42 & -- & .45 & -- & -- \\
\hline Nitrogen, total (mg/L as N) & .162 & .45 & .16 & .561 & -- & -- \\
\hline Orthophosphate, dissolved (mg/L as P) & .011 & $<.01$ & $<.01$ & .013 & -- & -- \\
\hline Lead, dissolved ( $\mu \mathrm{g} / \mathrm{L})$ & $<1$ & $<1$ & $<1$ & $<1$ & -- & (15) \\
\hline Manganese, dissolved ( $\mu \mathrm{g} / \mathrm{L})$ & 2.2 & 4.7 & 15.1 & 1.6 & -- & (b50) \\
\hline Zinc, dissolved $(\mu \mathrm{g} / \mathrm{L})$ & 1.7 & 8.1 & 1.8 & 3.1 & -- & $(2,000)$ \\
\hline Arsenic, total $(\mu \mathrm{g} / \mathrm{L})$ & $<1$ & 1.4 & $<2.6$ & 9.7 & -- & 150 \\
\hline Cadmium, total $(\mu \mathrm{g} / \mathrm{L})$ & $<1$ & $<1$ & $<.1$ & .6 & -- & ${ }^{\mathrm{c}} .5$ \\
\hline Copper, total $(\mu \mathrm{g} / \mathrm{L})$ & 1.3 & 9.2 & 1.4 & 34 & -- & $c_{20}$ \\
\hline Iron, total $(\mu \mathrm{g} / \mathrm{L})$ & 244 & 418 & 198 & 31,470 & -- & 1,000 \\
\hline Lead, total $(\mu \mathrm{g} / \mathrm{L})$ & $<1$ & 1.5 & $<1$ & 33 & -- & c9.7 \\
\hline Manganese, total $(\mu \mathrm{g} / \mathrm{L})$ & 15 & 23 & 19 & 1,143 & -- & -- \\
\hline Zinc, total $(\mu \mathrm{g} / \mathrm{L})$ & 40 & 40 & 11 & 121 & -- & $c^{2} 252$ \\
\hline
\end{tabular}

${ }^{\text {aN }}$ atural $\mathrm{pH}$ outside this range must be maintained.

${ }^{\mathrm{b}}$ Secondary standard based on aesthetic properties such as taste, odor, and staining.

cTable value standard calculated for a hardness of $240 \mathrm{mg} / \mathrm{L}$ for trace elements or $\mathrm{pH}$ of 8.4 at $20^{\circ} \mathrm{C}$ for ammonia. 
Table 27. Summary of selected water-quality data for ground-water sites in the Little Bighorn Battlefield National Monument, Montana.

[human-health standards from www.deq.state.mt.us/wqinfo/Standards/Index.asp; <, less than; --, not reported; ${ }^{\circ} \mathrm{C}$, degrees Celsius; mg/L, milligrams per liter; $\mu \mathrm{S} / \mathrm{cm}$, microsiemens per centimeter at $25^{\circ} \mathrm{C} ; \mathrm{CaCO}_{3}$, calcium carbonate; $\mathrm{N}$, nitrogen; $\mathrm{P}$, phosphorous; $\mu \mathrm{g} / \mathrm{L}$, micrograms per liter]

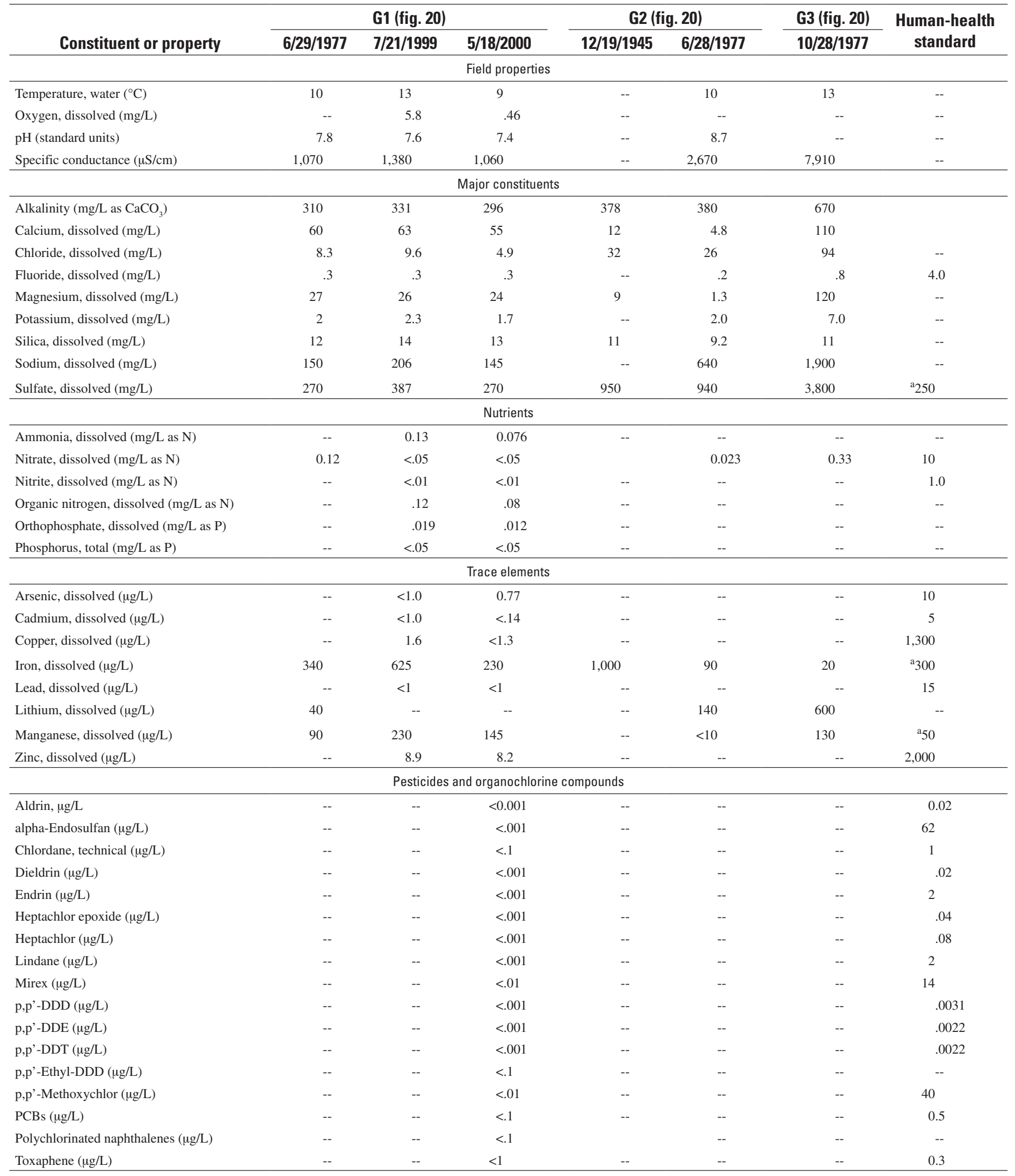

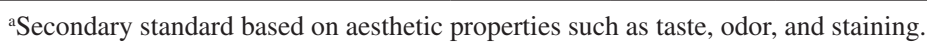




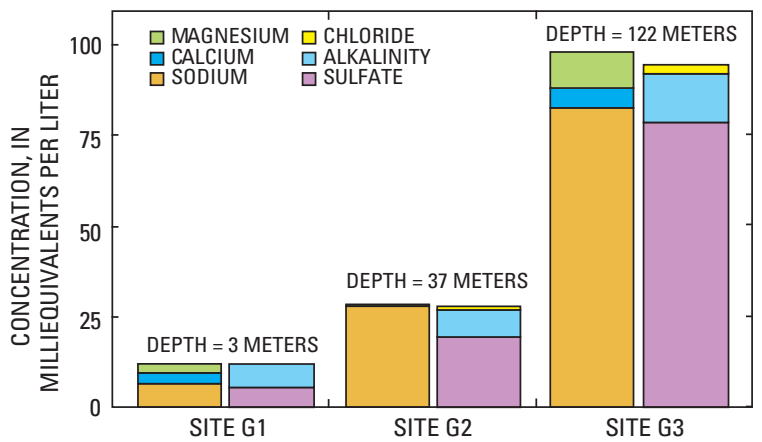

Figure 23. Variation in water quality with depth at selected ground-water wells in Little Bighorn National Battlefield Monument, Montana, 1977.

\section{Summary and Suggestions for Vital Signs Monitoring}

Review of historical records reveals few water-quality data are available for LIBI. From 1945 to 2000, a total of 11 samples were collected at 2 sites on the Little Bighorn River and 3 ground-water wells. Each site was sampled one to three times during the period of record with the exception of site S1 on the Little Bighorn River, which was sampled four times during 1999 and 2000. A streamflow-gaging station has been operated by the NPS on the Little Bighorn River (station 453400107263001) along the park boundary since November 1999, and water levels were measured annually at one well in the park during 1977 to 1999 . Surface water is a calciumbicarbonate-sulfate type and major constituents primarily are derived from natural weathering processes. Agricultural activities upstream from the park may affect nutrient and sediment concentrations in surface water, particularly during periods in increased streamflow. Because of a lack of long-term monitoring data, it was not possible to identify temporal trends in the water quality of the river. The reach of river in the park is classified as B-2, which is suitable for domestic water supply, recreation, aquatic life (salmonid fishes), and agricultural and industrial water supply. The available water-quality data indicate the river in this reach meets Montana aquatic-life and human-health standards, except perhaps during periods of high sediment transport. The standard comparison, however, is based on relatively few samples and additional data would be needed to characterize water-quality conditions during high flow. Ground water is a sodium-bicarbonate-sulfate type with elevated dissolved solid concentrations (more than $800 \mathrm{mg} / \mathrm{L}$ ). Nutrient concentrations in ground water were low, indicating minimal effects from agricultural activities outside the park. None of the ground-water samples exceeded human-health standards, although several exceeded the secondary standards for manganese and iron.

Effects of agricultural activities outside the park on nutrient, bacteria, and sediment concentrations likely are the main water-quality issues for the Little Bighorn River. Based on a review of the historical data, the following suggestions are provided for consideration in designing a water-quality monitoring plan for LIBI.

- Establish a long-term water-quality monitoring station or "sentinel site" at the Little Bighorn River (station 453400107263001). Monitor for core field properties, nutrients, sediment, and indicator bacteria during different hydrologic conditions and occasionally for major constituents and trace elements.

- Maintain the NPS streamflow-gaging station at the Little Bighorn River (station 453400107263001).

- Establish routine water-quality and water-level monitoring at existing ground-water wells. This information would be useful for investigating the effects of human stressors such as agriculture and climate change on ground-water resources.

\section{Rocky Mountain National Park}

\section{Environmental Setting and Geology}

Rocky Mountain National Park (ROMO) is located in the Colorado Front Range in north-central Colorado approximately $80 \mathrm{~km}$ northeast of Denver, Colorado (fig. 24). The park covers $1,075 \mathrm{~km}^{2}$ and is characterized by steep, mountainous terrain with elevations ranging from 2,323 $\mathrm{m}$ at the eastern park boundary to $4,343 \mathrm{~m}$ along the Continental Divide. Three major bioregions are present in ROMO including alpine tundra, subalpine forest, and montane forest (Baron, 1992). Vegetation is dominated by spruce-fir forests between 2,860 and 3,300 $\mathrm{m}$ and alpine tundra communities at higher elevations (Baron, 1992). The area is characterized by a continental climate with long, cold winters and a short growing season (1 to 3 months). Mean monthly air temperatures at $2,500 \mathrm{~m}$ ranges from $-4.4^{\circ} \mathrm{C}$ in January to $15.5^{\circ} \mathrm{C}$ in July (http://www.wrcc.dri.edu/cgi-bin/cliMAIN.pl?coalle, accessed November 2006) and at $3,160 \mathrm{~m}$ ranges from $-6.0^{\circ} \mathrm{C}$ in January to $13.8{ }^{\circ} \mathrm{C}$ in July (Baron, 1992). Precipitation varies considerably with elevation, with mean annual precipitation ranging from $40 \mathrm{~cm}$ at $2,500 \mathrm{~m}$ to $100 \mathrm{~cm}$ at $3,160 \mathrm{~m}$ (http:// nadp.sws.uiuc.edu/, accessed November 2006). More than one-half of the precipitation occurs as snow that accumulates in a seasonal snowpack between October and April. Winter precipitation is associated with synoptic weather systems with westerly airflow, and summer precipitation is associated with convective air masses originating primarily from the southwest and southeast (Baron and Denning, 1993).

Bedrock in ROMO predominantly is granite, gneiss, and schist of Proterozoic age (Braddock and Cole, 1990). The mineralogy of the Proterozoic rocks is similar, and consists of quartz, biotite, plagioclase, microcline and sillimanite. Some Tertiary lava flows crop out in the northwestern corner of the park. During the Pleistocene, valley glaciers covered the region carving the alpine cirques, glacial moraines, and $\mathrm{U}$-shaped valleys that give the park its spectacular scenery. 


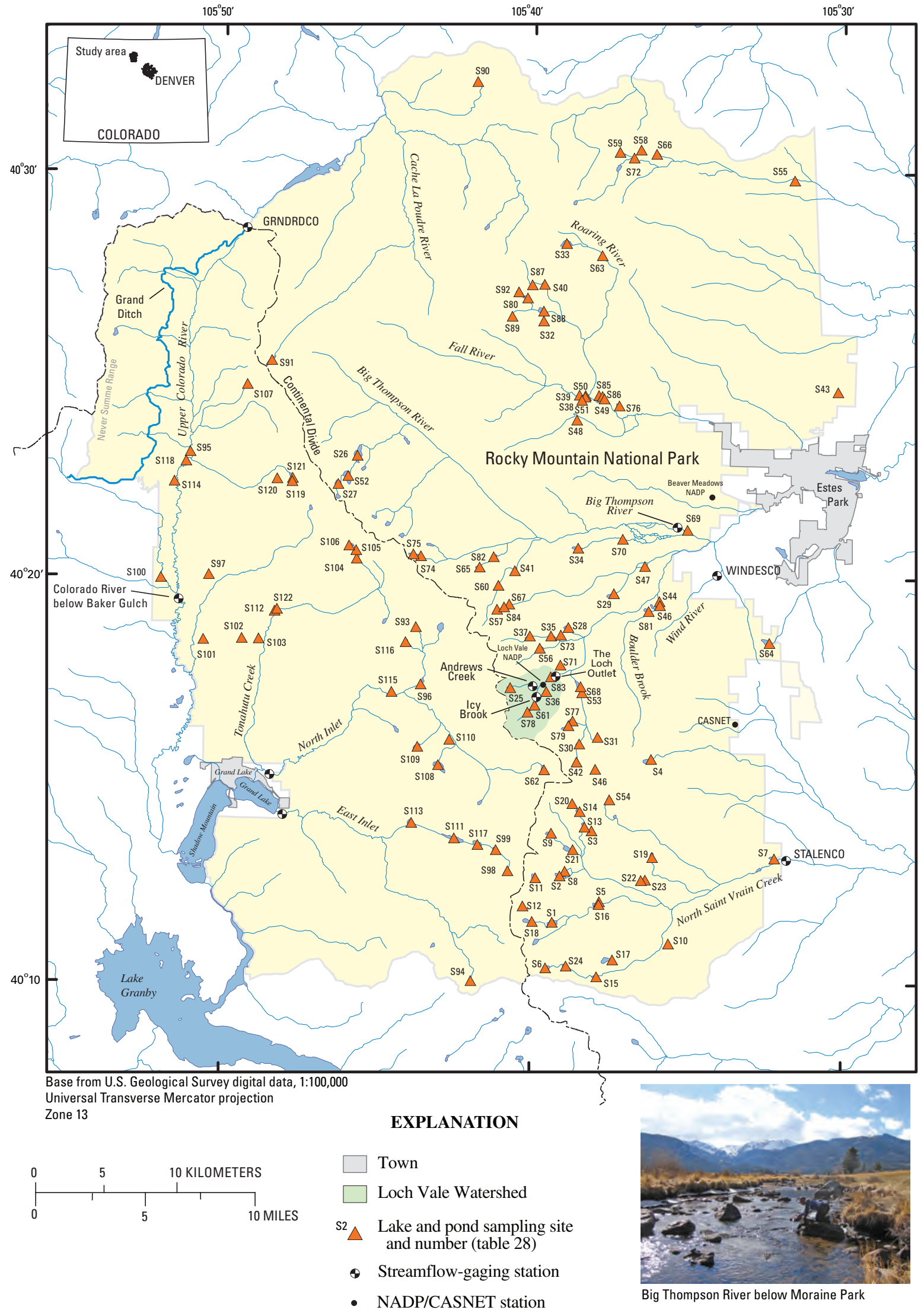

Figure 24. Location of lake and pond sampling sites in Rocky Mountain National Park, Colorado. 
The Pinedale glaciation, the last of three separate Pleistocene advances, left extensive till deposits at elevations between 2,400 and 3,200 m (Madole, 1976). Several Neoglacial advances during the Holocene formed moraines, rock glaciers, talus, and debris flows present at higher elevations (3,300 to $3,750 \mathrm{~m}$ ) in the park. The few small glaciers that exist in the park today are remnants of the most recent glaciation, but have been receding since the mid-1800s (Baron, 1992).

Approximately 95 percent of ROMO is designated or recommended as wilderness, and human activities are limited in these areas. Land-use activities in other areas of the park are related to tourism and park administration, and include the road system, campgrounds, picnic areas, liveries, visitor centers, and administrative buildings and park housing. The park has approximately 3 million visitors each year, most of whom visit the park between July and September (Britten and others, 2006). Water supply for the park is obtained from a system of 4 surface-water sources and 15 ground-water sources. The CDPHE requires monitoring for microbiological, chemical, physical, and radiological contaminants in public water systems (http://www.cdphe.state.co.us/wq/drinkingwater/index. html, accessed November 2006). The monitoring frequency is dependent on the type of water system, the water source, and the presence of contaminant-generating activities in the area surrounding the water source. The park has a few small septic systems but the majority of wastewater is treated by municipal treatment facilities outside of the park boundary in Estes Park and Grand Lake.

ROMO holds Federal reserved water rights for surface and ground water dating back to 1915 for purposes of conserving and maintaining park resources in an unimpaired condition. One exception is the Grand Ditch water diversion project, which was constructed before the establishment of ROMO. The project consists of a 24-km long drainage ditch that is carved into the eastern slope of the Never Summer Range (fig. 24). The ditch intercepts streamflow from 13 upstream drainage areas from mid June to mid September, and diverts the water to the east side of the Continental Divide (Woods and others, 2006). The seasonal diversion of surface water causes substantial declines in water levels in wetlands downstream from the ditch (Woods and others, 2006). In May 2003, the ditch breached because of ice and debris blockage and 46,000 cubic meters of sediment was washed into the upper Colorado River drainage causing damage to wetlands, forests, trails, bridges, and campsites. Four water-storage reservoirs including Bluebird Lake (S1 in fig. 24), Lawn Lake (S63), Pear Lake (S17), and Sandbeach Lake (S19) existed in the park before its establishment in 1915. In July 1982, the dam on Lawn Lake failed, resulting in substantial property damage to downstream communities and the loss of three lives. As a result, the dams were removed from all four reservoirs during 1988 to 1990 and 2002 and lakes were restored to pre-dam levels (http:// www.nps.gov/romo/naturescience/hydrologicactivity.htm, accessed November 2006).

Air pollution is arguably the main natural resource issue for ROMO. The park is situated less than $70 \mathrm{~km}$ to the west of the Front Range urban corridor, which contains the most concentrated population density in the Rocky Mountain region, as well as directly west of large expanses of cropland and pasture on the plains of eastern Colorado. These urban and agricultural areas are sources of air pollution that are causing visibility degradation, increased ozone levels, and acidic deposition (nitrogen and sulfur) in the park (http://www.nps. gov/romo/naturescience/airquality.htm, accessed November 2006). Perhaps the most serious of these air-pollution issues is atmospheric deposition of nitrogen to high-elevation areas of the park. Nitrogen deposition rates have been increasing in the park during the past two decades, and current year levels [3-4 kilograms of nitrogen per hectare per year ( $\mathrm{kg} \mathrm{N} / \mathrm{ha} / \mathrm{yr})$ ] are 15-20 times greater than the estimated natural background level (Baron, 2006). Ecosystem effects from nitrogen deposition have been documented on the east side of the park and include changes in the type and abundance of aquatic plant species (diatoms), chronically elevated levels of nitrate in surface waters, elevated levels of nitrogen in spruce tree chemistry, and accumulation of nitrogen in forest soils (Burns, 2004). ROMO has proposed that a reduction in the deposition rate to $1.5 \mathrm{~kg} \mathrm{~N} / \mathrm{ha} / \mathrm{yr}$ would be needed to restore healthy ecosystems and is currently working on emission reduction strategies with the State of Colorado and USEPA to achieve this management goal. Additional information on the effects of atmospheric deposition in ROMO is available on the CDPHE web site at http://www.cdphe.state.co.us/ap/rmnp.html (accessed November 2006).

\section{Water Resources}

Drainages on the east side of ROMO form the headwaters of the Big Thompson River, Saint Vrain Creek, and Cache La Poudre River; those on the west side drain into the Upper Colorado River system. The park contains nearly $800 \mathrm{~km}$ of perennial streams and more than 150 lakes, most of which are above 3,000 $\mathrm{m}$ in the subalpine and alpine zones (Britten and others, 2006). The USGS has operated as many as 14 streamflow-gaging stations in or adjacent to the boundaries of the park. Of these, four stations currently (2007) are active including Andrews Creek in Loch Vale (USGS station 401723105400000), Icy Brook in Loch Vale (USGS station 401707105395000), Big Thompson River at Moraine Park (USGS station 402114105350101), and the Colorado River below Baker Gulch (USGS station 09010500) (fig. 24). The Big Thompson River at Moraine Park is part of the USGS Hydrologic Benchmark Network, a National program for monitoring streamflow and water quality in areas that are minimally affected by human activities (Murdoch and others, 2005).

The Andrews Creek and Icy Brook gages are operated as part of the USGS Water, Energy, and Biogeochemical Budgets Program (Baedecker and Friedman, 2000; Clow and others, 2000). These two gages are located in the Loch Vale Watershed (LVWS), which is an ecological research area that was established by the NPS in 1981 to study biogeochemical 
and hydrologic processes in alpine and subalpine ecosystems (Baron, 1992). The NPS also has a gage in LVWS at the outlet of The Loch that has been in operation since 1983. LVWS is a 6.6- $\mathrm{km}^{2}$ watershed that drains subalpine and alpine terrain on the east side of the Continental Divide (fig. 24). The watershed contains two alpine streams (Andrews Creek and Icy Brook), three lakes (Sky Pond, Lake of Glass, and The Loch), and two small glaciers. More details about LVWS and its research programs are presented in Baron (1992) and Clow and others (2000) and at the following two web sites-http://www.nrel. colostate.edu/projects/lvws/ and http://co.water.usgs.gov/ lochvale/index.html (accessed November 2006).

The Colorado Division of Water Resource operates several gages just outside the park boundary including North Saint Vrain Creek near Allenspark (station STALENCO), Wind River near Estes Park (station WINDESCO), and Grand River Ditch at La Poudre Pass (station GRNDRDCO) (fig. 24). Data for these stations are available at http://water.state.co.us/ pubs/datasearch.asp (accessed November 2006). The Northern Colorado Water Conservation District operates seasonal gages near the mouth of East Inlet Creek and North Inlet Creek on the west side of the park (http://www.ncwcd.org/datareports/ westflow.asp, accessed November 2006).

The longest continuously operated station in the park is the Colorado River below Baker Gulch (USGS station 09010500), which began operation in 1953 (fig. 24). The range of daily streamflow values for this station from 1986 to 2005 is shown in figure 25. Peak streamflow occurs in late May or early June during spring snowmelt, and streamflow increases by two to three orders of magnitude over winter streamflows. Nearly 65 percent of the annual streamflow occurs in May and June. Streamflows decrease rapidly after June, and reaches a minimum in late winter when streamflow is sustained by slow release of ground water.

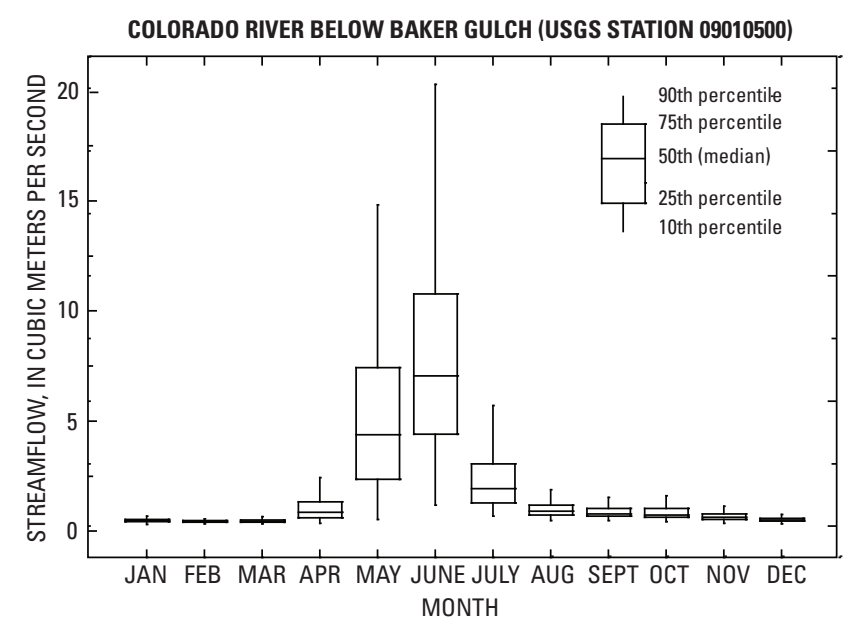

Figure 25. Daily streamflow range at Colorado River near Baker Gulch (USGS streamflow-gaging station 09010500) in Rocky Mountain National Park, Colorado, 1986-2005.
In mountainous areas such as ROMO there generally are two different ground-water systems; bedrock aquifers in the mountain block, and shallow localized aquifers in alluvium and glacial till. In ROMO, the hydrologic characteristics of mountain block aquifers are virtually unknown and there have been only a few published studies of shallow aquifer systems, most of which have been related to wetlands. Bachman (1994) made water-level measurements at 20 shallow wells (less than $1.9 \mathrm{~m}$ ) in LVWS from 1991 to 1993 to study ground-water movement in subalpine wetlands. Cooper and others (1998) measured water levels at 50 shallow wells (less than $2.5 \mathrm{~m}$ ) in a wetland along Tonahutu Creek from 1987 to 1994 to determine the effect of hydrologic restoration activities in the wetland. Woods and others (2006) measured water levels in 18 shallow wells (less than $1.6 \mathrm{~m}$ ) in a small wetland along the upper Colorado River to study controls on wetland water levels and the effect of the upstream water diversions related to the Grand Ditch. These studies concluded that seepage of surface water was an important wetland water source, and suggested these systems could be sensitive to climate variability or changes in hydrology related to water diversions. Clow and others (2004) estimated ground-water occurrence and contributions to streamflow using hydrogeomorphologic mapping, seismic refraction measurements, tracer tests, and porosity and permeability estimates in LWVS. The study concluded that talus deposits formed at the base of cliffs are the primary ground-water reservoir in alpine areas, and that discharge from these deposits accounts for more than 75 percent of streamflow during summer storms and the winter base-flow period. The second largest ground-water reservoir in alpine areas was determined to be ice stored in permafrost in talus and rock glaciers.

The park has two NADP stations, one in LVWS at an elevation of 3,159 $\mathrm{m}$ and one at Beaver Meadows at an elevation of 2,490 m (fig. 24). The NADP is a national network of precipitation monitoring sites that measures weekly precipitation chemistry and precipitation amount; data for the network are available at http://nadp.sws.uiuc.edu/ (accessed November 2006). The sites in ROMO were established in the early 1980 s to monitor the effects of air pollution on ecological resources including vegetation, water quality, and soils (http://www2. nature.nps.gov/air/index.cfm, accessed November 2006). The park also has a CASNET station located near the south-eastern park boundary at $2,800 \mathrm{~m}$. CASTNET is a national network of air monitoring sites that provide estimates of dry deposition of sulfur and nitrogen compounds and concentrations of groundlevel ozone (http://www.epa.gov/castnet/, accessed November 2006). The CASNET station in ROMO is operated by the NPS and was added to the network in 1995.

\section{Surface-Water Quality}

Water-quality data were collected and compiled for 493 surface-water sites within the boundaries of ROMO including 122 lakes and ponds, 83 springs, and 288 streams. For 
Table 28. Lake and pond sampling sites in Rocky Mountain National Park, Colorado.

[no., number; identification numbers and station names from USEPA STORET and USGS NWIS]

\begin{tabular}{|c|c|c|c|c|c|c|c|}
\hline $\begin{array}{l}\text { Site no. } \\
\text { (fig. 24) }\end{array}$ & Identification number & Station name & Latitude & Longitude & $\begin{array}{c}\text { Elevation } \\
\text { (meters) }\end{array}$ & Type & $\begin{array}{c}\text { No. } \\
\text { samples }\end{array}$ \\
\hline S1 & 4E1-051 & Bluebird Lake & $40^{\circ} 11^{\prime} 30^{\prime \prime}$ & $105^{\circ} 39^{\prime} 17^{\prime \prime}$ & 3,348 & Lake & 4 \\
\hline S2 & 4E1-030 & Box Lake & $40^{\circ} 12^{\prime} 47^{\prime \prime}$ & $105^{\circ} 38^{\prime} 55^{\prime \prime}$ & 3,276 & Lake & 2 \\
\hline S3 & ROMO_JR_CAP29 & Castle Lake North of the Outlet & $40^{\circ} 13^{\prime} 47^{\prime \prime}$ & $105^{\circ} 38^{\prime} 04^{\prime \prime}$ & 3,414 & Lake & 1 \\
\hline S4 & 401533105360800 & Chasm Lake & $40^{\circ} 15^{\prime} 33^{\prime \prime}$ & $105^{\circ} 36^{\prime} 10^{\prime \prime}$ & 3,591 & Lake & 16 \\
\hline S5 & ROMO_JR_CAP30 & Chickadee Pond near Southwest Corner & $40^{\circ} 12^{\prime} 02^{\prime \prime}$ & $105^{\circ} 37^{\prime} 49^{\prime \prime}$ & 3,055 & Pond & 1 \\
\hline S6 & 401025105392000 & Cony Lake & $40^{\circ} 10^{\prime} 24^{\prime \prime}$ & $105^{\circ} 39^{\prime} 27^{\prime \prime}$ & 3,505 & Lake & 1 \\
\hline S7 & 401307105320900 & Copeland Lake & $40^{\circ} 13^{\prime} 07^{\prime \prime}$ & $105^{\circ} 32^{\prime} 11^{\prime \prime}$ & 2,533 & Lake & 7 \\
\hline S8 & 401137105384900 & Eagle Lake & $40^{\circ} 12^{\prime} 40^{\prime \prime}$ & $105^{\circ} 39^{\prime} 05^{\prime \prime}$ & 3,298 & Lake & 1 \\
\hline S9 & 401343105392201 & Falcon Lake & $40^{\circ} 13^{\prime} 43^{\prime \prime}$ & $105^{\circ} 39^{\prime} 22^{\prime \prime}$ & 3,376 & Lake & 2 \\
\hline S10 & 4E1-038 & Finch Lake & $40^{\circ} 10^{\prime} 60^{\prime \prime}$ & $105^{\circ} 35^{\prime} 35^{\prime \prime}$ & 3,023 & Lake & 25 \\
\hline S11 & 401237105394600 & Frigid Lake & $40^{\circ} 12^{\prime} 37^{\prime \prime}$ & $105^{\circ} 39^{\prime} 52^{\prime \prime}$ & 3,600 & Lake & 1 \\
\hline S12 & 401155105401700 & Isolation Lake & $40^{\circ} 11^{\prime} 55^{\prime \prime}$ & $105^{\circ} 40^{\prime} 17^{\prime \prime}$ & 3,655 & Lake & 1 \\
\hline S13 & 401352105381801 & Lion Lake \#1 & $40^{\circ} 13^{\prime} 52^{\prime \prime}$ & $105^{\circ} 38^{\prime} 18^{\prime \prime}$ & 3,375 & Lake & 5 \\
\hline S14 & 401415105382701 & Lion Lake \#2 & $40^{\circ} 14^{\prime} 15^{\prime \prime}$ & $105^{\circ} 38^{\prime} 27^{\prime \prime}$ & 3,481 & Lake & 2 \\
\hline S15 & 401013105374900 & Lower Hutcheson & $40^{\circ} 10^{\prime} 11^{\prime \prime}$ & $105^{\circ} 37^{\prime} 54^{\prime \prime}$ & 3,316 & Lake & 2 \\
\hline S16 & 401158105375001 & Ouzel Lake & $40^{\circ} 11^{\prime} 58^{\prime \prime}$ & $105^{\circ} 37^{\prime} 50^{\prime \prime}$ & 3,056 & Lake & 4 \\
\hline S17 & 401036105372301 & Pear Lake & $40^{\circ} 10^{\prime} 36^{\prime \prime}$ & $105^{\circ} 37^{\prime} 23^{\prime \prime}$ & 3,225 & Lake & 5 \\
\hline S18 & 401132105395801 & Pipit Lake & $40^{\circ} 11^{\prime} 32^{\prime \prime}$ & $105^{\circ} 39^{\prime} 58^{\prime \prime}$ & 3,486 & Lake & 3 \\
\hline S19 & 4E1-033 & Sandbeach Lake & $40^{\circ} 13^{\prime} 08^{\prime \prime}$ & $105^{\circ} 36^{\prime} 07^{\prime \prime}$ & 3,136 & Lake & 7 \\
\hline S20 & 401424105383301 & Snowbank Lake & $40^{\circ} 14^{\prime} 24^{\prime \prime}$ & $105^{\circ} 38^{\prime} 33^{\prime \prime}$ & 3,513 & Lake & 3 \\
\hline $\mathrm{S} 21$ & 401319105384001 & Thunder Lake & $40^{\circ} 13^{\prime} 19^{\prime \prime}$ & $105^{\circ} 38^{\prime} 40^{\prime \prime}$ & 3,225 & Lake & 4 \\
\hline S22 & ROMO_JR_CAP38 & Twin Lakes (Lower) & $40^{\circ} 12^{\prime} 34^{\prime \prime}$ & $105^{\circ} 36^{\prime} 20^{\prime \prime}$ & 3,000 & Lake & 1 \\
\hline S23 & ROMO_JR_CAP39 & Twin Lakes (Upper) & $40^{\circ} 12^{\prime} 34^{\prime \prime}$ & $105^{\circ} 36^{\prime} 28^{\prime \prime}$ & 3,000 & Lake & 1 \\
\hline S24 & ROMO_JR_CAP40 & Upper Hutcheson Lake & $40^{\circ} 10^{\prime} 26^{\prime \prime}$ & $105^{\circ} 38^{\prime} 52^{\prime \prime}$ & 3,414 & Lake & 1 \\
\hline S25 & 401723105394803 & Andrews Tarn & $40^{\circ} 17^{\prime} 18^{\prime \prime}$ & $105^{\circ} 40^{\prime} 43^{\prime \prime}$ & 3,471 & Lake & 7 \\
\hline S26 & 4E1-014 & Arrowhead Lake & $40^{\circ} 22^{\prime} 60^{\prime \prime}$ & $105^{\circ} 45^{\prime} 42^{\prime \prime}$ & 3,398 & Lake & 2 \\
\hline S27 & 402222105461100 & Azure Lake & $40^{\circ} 22^{\prime} 18^{\prime \prime}$ & $105^{\circ} 46^{\prime} 19^{\prime \prime}$ & 3,505 & Lake & 1 \\
\hline S28 & 401843105384700 & Bear Lake & $40^{\circ} 18^{\prime} 47^{\prime \prime}$ & $105^{\circ} 38^{\prime} 52^{\prime \prime}$ & 2,883 & Lake & 14 \\
\hline S29 & 401938105372200 & Bierstadt Lake & $40^{\circ} 19^{\prime} 38^{\prime \prime}$ & $105^{\circ} 37^{\prime} 24^{\prime \prime}$ & 2,871 & Lake & 4 \\
\hline S30 & 4E1-025 & Black Lake & $40^{\circ} 15^{\prime} 55^{\prime \prime}$ & $105^{\circ} 38^{\prime} 29^{\prime \prime}$ & 3,239 & Lake & 32 \\
\hline S31 & 401602105375300 & Blue Lake & $40^{\circ} 16^{\prime} 05^{\prime \prime}$ & $105^{\circ} 37^{\prime} 54^{\prime \prime}$ & 3,399 & Lake & 4 \\
\hline S32 & ROMO_JR_CAP04 & Chipmunk Lake & $40^{\circ} 26^{\prime} 21^{\prime \prime}$ & $105^{\circ} 39^{\prime} 43^{\prime \prime}$ & 3,250 & Lake & 1 \\
\hline S33 & GA04 & Crystal Lake & $40^{\circ} 28^{\prime} 16^{\prime \prime}$ & $105^{\circ} 39^{\prime} 00^{\prime \prime}$ & 3,511 & Lake & 2 \\
\hline S34 & 402046105382800 & Cub Lake & $40^{\circ} 20^{\prime} 45^{\prime \prime}$ & $105^{\circ} 38^{\prime} 34^{\prime \prime}$ & 2,621 & Lake & 2 \\
\hline S35 & 401835105392300 & Dream Lake & $40^{\circ} 18^{\prime} 35^{\prime \prime}$ & $105^{\circ} 39^{\prime} 25^{\prime \prime}$ & 3,018 & Lake & 23 \\
\hline S36 & 401715105394201 & Embryo Pond & $40^{\circ} 17^{\prime} 15^{\prime \prime}$ & $105^{\circ} 39^{\prime} 42^{\prime \prime}$ & 3,169 & Lake & 5 \\
\hline S37 & 401835105395800 & Emerald Lake & $40^{\circ} 18^{\prime} 35^{\prime \prime}$ & $105^{\circ} 40^{\prime} 06^{\prime \prime}$ & 3,078 & Lake & 44 \\
\hline S38 & ROMO_USFW_FAN & Fan Lake & $40^{\circ} 24^{\prime} 32^{\prime \prime}$ & $105^{\circ} 38^{\prime} 33^{\prime \prime}$ & 2,605 & Pond & 7 \\
\hline S39 & 402430105381700 & Fan Lake \#2 & $40^{\circ} 24^{\prime} 31^{\prime \prime}$ & $105^{\circ} 38^{\prime} 21^{\prime \prime}$ & 2,603 & Pond & 1 \\
\hline S40 & GB10 & Fay Lakes & $40^{\circ} 27^{\prime} 15^{\prime \prime}$ & $105^{\circ} 39^{\prime} 42^{\prime \prime}$ & 3,353 & Lake & 2 \\
\hline S41 & 402011105403801 & Fern Lake & $40^{\circ} 20^{\prime} 11^{\prime \prime}$ & $105^{\circ} 40^{\prime} 36^{\prime \prime}$ & 2,903 & Lake & 7 \\
\hline S42 & 401533105382900 & Frozen Lake & $40^{\circ} 15^{\prime} 33^{\prime \prime}$ & $105^{\circ} 38^{\prime} 31^{\prime \prime}$ & 3,530 & Lake & 7 \\
\hline S43 & 402438105301000 & Gem Lake & $40^{\circ} 24^{\prime} 38^{\prime \prime}$ & $105^{\circ} 30^{\prime} 12^{\prime \prime}$ & 2,688 & Lake & 1 \\
\hline S44 & 401927105355500 & Glacier Basin Primenet & $40^{\circ} 19^{\prime} 27^{\prime \prime}$ & $105^{\circ} 35^{\prime} 56^{\prime \prime}$ & 2,627 & Pond & 8 \\
\hline S45 & 401925105355500 & Glacier Basin PRIMENET New & $40^{\circ} 19^{\prime} 25^{\prime \prime}$ & $105^{\circ} 35^{\prime} 55^{\prime \prime}$ & 2,621 & Pond & 12 \\
\hline S46 & 401518105375500 & Green Lake & $40^{\circ} 15^{\prime} 18^{\prime \prime}$ & $105^{\circ} 37^{\prime} 58^{\prime \prime}$ & 3,520 & Lake & 6 \\
\hline S47 & 402019105362200 & Hallowell Park & $40^{\circ} 20^{\prime} 19^{\prime \prime}$ & $105^{\circ} 36^{\prime} 24^{\prime \prime}$ & 2,548 & Pond & 1 \\
\hline S48 & 402357381053822 & Hidden Valley & $40^{\circ} 23^{\prime} 57^{\prime \prime}$ & $105^{\circ} 38^{\prime} 40^{\prime \prime}$ & 2,548 & Pond & 1 \\
\hline S49 & 402427105374900 & Horseshoe Park & $40^{\circ} 24^{\prime} 27^{\prime \prime}$ & $105^{\circ} 37^{\prime} 51^{\prime \prime}$ & 2,606 & Pond & 3 \\
\hline
\end{tabular}


Table 28. Lake and pond sampling sites in Rocky Mountain National Park, Colorado._Continued

[no., number; identification numbers and station names from USEPA STORET and USGS NWIS]

\begin{tabular}{|c|c|c|c|c|c|c|c|}
\hline $\begin{array}{l}\text { Site no. } \\
\text { (fig. 24) }\end{array}$ & Identification number & Station name & Latitude & Longitude & $\begin{array}{c}\text { Elevation } \\
\text { (meters) }\end{array}$ & Type & $\begin{array}{c}\text { No. } \\
\text { samples }\end{array}$ \\
\hline S50 & ROMO_USFW_HP1 & Horseshoe Park Lake \#1 & $40^{\circ} 24^{\prime} 29^{\prime \prime}$ & $105^{\circ} 38^{\prime} 22^{\prime \prime}$ & 2,602 & Pond & 7 \\
\hline S51 & ROMO_USFW_HP3 & Horseshoe Park Lake \#3 & $40^{\circ} 24^{\prime} 24^{\prime \prime}$ & $105^{\circ} 38^{\prime} 28^{\prime \prime}$ & 2,602 & Pond & 3 \\
\hline S52 & 402236105455200 & Inkwell Lake & $40^{\circ} 22^{\prime} 30^{\prime \prime}$ & $105^{\circ} 45^{\prime} 60^{\prime \prime}$ & 3,493 & Lake & 1 \\
\hline S53 & ROMO_JR_CAP13 & Jewell Lake & $40^{\circ} 17^{\prime} 11^{\prime \prime}$ & $105^{\circ} 38^{\prime} 24^{\prime \prime}$ & 3,036 & Lake & 4 \\
\hline S54 & 4E1-032 & Keplinger Lake & $40^{\circ} 14^{\prime} 33^{\prime \prime}$ & $105^{\circ} 37^{\prime} 30^{\prime \prime}$ & 3,564 & Lake & 2 \\
\hline S55 & 402950105313700 & Kettle Tarn & $40^{\circ} 29^{\prime} 51^{\prime \prime}$ & $105^{\circ} 31^{\prime} 38^{\prime \prime}$ & 2,810 & Pond & 19 \\
\hline S56 & $4 \mathrm{E} 1-022$ & Lake Haiyaha & $40^{\circ} 18^{\prime} 17^{\prime \prime}$ & $105^{\circ} 39^{\prime} 47^{\prime \prime}$ & 3,117 & Lake & 37 \\
\hline S57 & 401915105410900 & Lake Helene & $40^{\circ} 19^{\prime} 14^{\prime \prime}$ & $105^{\circ} 41^{\prime} 10^{\prime \prime}$ & 3,219 & Lake & 2 \\
\hline S58 & 403033105363801 & Lake Husted & $40^{\circ} 30^{\prime} 35^{\prime \prime}$ & $105^{\circ} 36^{\prime} 36^{\prime \prime}$ & 3,381 & Lake & 43 \\
\hline S59 & 403028105371301 & Lake Louise & $40^{\circ} 30^{\prime} 29^{\prime \prime}$ & $105^{\circ} 37^{\prime} 14^{\prime \prime}$ & 3,362 & Lake & 35 \\
\hline S60 & ROMO_JR_CAP24 & Lake Odessa (North End) & $40^{\circ} 19^{\prime} 49^{\prime \prime}$ & $105^{\circ} 41^{\prime} 07^{\prime \prime}$ & 3,055 & Lake & 2 \\
\hline S61 & 401655105395401 & Lake Of Glass & $40^{\circ} 16^{\prime} 53^{\prime \prime}$ & $105^{\circ} 39^{\prime} 53^{\prime \prime}$ & 3,298 & Lake & 153 \\
\hline S62 & 4E1-026 & Lake Powell & $40^{\circ} 15^{\prime} 17^{\prime \prime}$ & $105^{\circ} 39^{\prime} 37^{\prime \prime}$ & 3,520 & Lake & 2 \\
\hline S63 & ROMO_JR_CAP15 & Lawn Lake & $40^{\circ} 27^{\prime} 59^{\prime \prime}$ & $105^{\circ} 37^{\prime} 51^{\prime \prime}$ & 3,353 & Lake & 3 \\
\hline S64 & 402657110232200 & Lily Pond & $40^{\circ} 18^{\prime} 26^{\prime \prime}$ & $105^{\circ} 32^{\prime} 22^{\prime \prime}$ & 2,900 & Pond & 9 \\
\hline S65 & 402017105414400 & Loomis Lake & $40^{\circ} 20^{\prime} 16^{\prime \prime}$ & $105^{\circ} 41^{\prime} 44^{\prime \prime}$ & 3,118 & Lake & 1 \\
\hline S66 & 403029105360400 & Lost Lake & $40^{\circ} 30^{\prime} 29^{\prime \prime}$ & $105^{\circ} 36^{\prime} 06^{\prime \prime}$ & 3,266 & Lake & 8 \\
\hline S67 & 401921105404600 & Marigold Pond Outflow & $40^{\circ} 19^{\prime} 21^{\prime \prime}$ & $105^{\circ} 40^{\prime} 46^{\prime \prime}$ & 3,255 & Pond & 2 \\
\hline S68 & 4E1-060 & Mills Lake & $40^{\circ} 17^{\prime} 20^{\prime \prime}$ & $105^{\circ} 38^{\prime} 27^{\prime \prime}$ & 3,032 & Lake & 65 \\
\hline S69 & 402113105346000 & Moraine Park, Lower & $40^{\circ} 21^{\prime} 13^{\prime \prime}$ & $105^{\circ} 35^{\prime} 02^{\prime \prime}$ & 2,454 & Pond & 1 \\
\hline S70 & 402059105370500 & Moraine Park, Upper & $40^{\circ} 20^{\prime} 59^{\prime \prime}$ & $105^{\circ} 37^{\prime} 07^{\prime \prime}$ & 2,474 & Pond & 13 \\
\hline S71 & 401752105390601 & Mystery Pond (No Name) & $40^{\circ} 17^{\prime} 52^{\prime \prime}$ & $105^{\circ} 39^{\prime} 06^{\prime \prime}$ & 3,092 & Pond & 1 \\
\hline S72 & 403023105364800 & North Fork Ponds & $40^{\circ} 30^{\prime} 23^{\prime \prime}$ & $105^{\circ} 36^{\prime} 50^{\prime \prime}$ & 3,350 & Pond & 1 \\
\hline S73 & ROMO_JR_CAP23 & Nymph Lake near Longs Peak Viewpod & $40^{\circ} 18^{\prime} 37^{\prime}$ & $105^{\circ} 39^{\prime} 06^{\prime \prime}$ & 2,969 & Lake & 1 \\
\hline S74 & 402032105433801 & Rainbow Lake (Lower) & $40^{\circ} 20^{\prime} 32^{\prime \prime}$ & $105^{\circ} 43^{\prime} 38^{\prime \prime}$ & 3,580 & Lake & 1 \\
\hline S75 & 402035105435201 & Rainbow Lake (Upper) & $40^{\circ} 20^{\prime} 35^{\prime \prime}$ & $105^{\circ} 43^{\prime} 52^{\prime \prime}$ & 3,613 & Lake & 1 \\
\hline S76 & 402416105371400 & Sheep Lake & $40^{\circ} 24^{\prime} 16^{\prime \prime}$ & $105^{\circ} 37^{\prime} 16^{\prime \prime}$ & 2,593 & Lake & 3 \\
\hline S77 & 401629105384000 & Shelf Lake & $40^{\circ} 16^{\prime} 29^{\prime \prime}$ & $105^{\circ} 38^{\prime} 42^{\prime \prime}$ & 3,420 & Lake & 3 \\
\hline S78 & 401642105400601 & Sky Pond & $40^{\circ} 16^{\prime} 39^{\prime \prime}$ & $105^{\circ} 40^{\prime} 07^{\prime \prime}$ & 3,319 & Lake & 267 \\
\hline S79 & 401622105384800 & Solitude Lake & $40^{\circ} 16^{\prime} 22^{\prime \prime}$ & $105^{\circ} 38^{\prime} 50^{\prime \prime}$ & 3,475 & Lake & 3 \\
\hline S 80 & 402653105400600 & Spectacle Lakes Southeast & $40^{\circ} 26^{\prime} 55^{\prime \prime}$ & $105^{\circ} 40^{\prime} 14^{\prime \prime}$ & 3,456 & Lake & 1 \\
\hline S81 & 401910105360800 & Sprague Lake & $40^{\circ} 19^{\prime} 12^{\prime \prime}$ & $105^{\circ} 36^{\prime} 17^{\prime \prime}$ & 2,646 & Lake & 2 \\
\hline S82 & 402032105411500 & Spruce Lake & $40^{\circ} 20^{\prime} 32^{\prime \prime}$ & $105^{\circ} 41^{\prime} 17^{\prime \prime}$ & 2,707 & Lake & 11 \\
\hline S83 & 4E1-061 & The Loch & $40^{\circ} 17^{\prime} 33^{\prime \prime}$ & $105^{\circ} 39^{\prime} 37^{\prime \prime}$ & 3,105 & Lake & 342 \\
\hline S84 & 401918105405300 & Two Rivers Lake & $40^{\circ} 19^{\prime} 17^{\prime \prime}$ & $105^{\circ} 40^{\prime} 57^{\prime \prime}$ & 3,261 & Lake & 2 \\
\hline S85 & ROMO_NPS_FLD_3 & Unnamed Pond F-Lawn Lake Alluvial Fan & $40^{\circ} 24^{\prime} 30^{\prime \prime}$ & $105^{\circ} 37^{\prime} 48^{\prime \prime}$ & 2,606 & Pond & 10 \\
\hline S86 & ROMO_NPS_FLD_4 & Unnamed Pond Q-Lawn Lake Alluvial Fan & $40^{\circ} 24^{\prime} 26^{\prime \prime}$ & $105^{\circ} 37^{\prime} 45^{\prime \prime}$ & 2,606 & Pond & 10 \\
\hline S87 & GB04 & Upper Fay Lakes & $40^{\circ} 27^{\prime} 15^{\prime \prime}$ & $105^{\circ} 40^{\prime} 05^{\prime \prime}$ & 3,425 & Lake & 1 \\
\hline S88 & 402636105394100 & Ypsilon Lake, Rocky Mountain National Park & $40^{\circ} 26^{\prime} 36^{\prime \prime}$ & $105^{\circ} 39^{\prime} 43^{\prime \prime}$ & 3,200 & Lake & 8 \\
\hline S89 & 4E1-013 & Chiquita Lake & $40^{\circ} 26^{\prime} 28^{\prime \prime}$ & $105^{\circ} 40^{\prime} 44^{\prime \prime}$ & 3,459 & Lake & 5 \\
\hline S90 & 4E1-053 & Mirror Lake & $40^{\circ} 32^{\prime} 15^{\prime \prime}$ & $105^{\circ} 41^{\prime} 54^{\prime \prime}$ & 3,361 & Lake & 4 \\
\hline S91 & 402522105482800 & Poudre Lake & $40^{\circ} 25^{\prime} 20^{\prime \prime}$ & $105^{\circ} 48^{\prime} 29^{\prime \prime}$ & 3,281 & Lake & 6 \\
\hline S92 & 4E1-012 & Spectacle Lakes (Northwest) & $40^{\circ} 27^{\prime} 04^{\prime \prime}$ & $105^{\circ} 40^{\prime} 32^{\prime \prime}$ & 3,459 & Lake & 3 \\
\hline S93 & $4 \mathrm{E} 1-035$ & (No Name) & $40^{\circ} 10^{\prime} 04^{\prime \prime}$ & $105^{\circ} 41^{\prime} 56^{\prime \prime}$ & 3,312 & Lake & 2 \\
\hline S94 & 4E1-019 & (No Name) & $40^{\circ} 18^{\prime} 47^{\prime \prime}$ & $105^{\circ} 43^{\prime} 47^{\prime \prime}$ & 3,373 & Lake & 2 \\
\hline S95 & 402305105510400 & Beaver Ponds Picnic Area & $40^{\circ} 23^{\prime} 05^{\prime \prime}$ & $105^{\circ} 51^{\prime} 06^{\prime \prime}$ & 2,743 & Pond & 1 \\
\hline S96 & 401723105433500 & Bench Lake & $40^{\circ} 17^{\prime} 23^{\prime \prime}$ & $105^{\circ} 43^{\prime} 37^{\prime \prime}$ & 3,094 & Lake & 2 \\
\hline S97 & 402005105502600 & Chickaree Lake & $40^{\circ} 20^{\prime} 03^{\prime \prime}$ & $105^{\circ} 50^{\prime} 28^{\prime \prime}$ & 2,808 & Lake & 2 \\
\hline S98 & 4E1-029 & Fifth Lake & $40^{\circ} 12^{\prime} 47^{\prime \prime}$ & $105^{\circ} 40^{\prime} 46^{\prime \prime}$ & 3,312 & Lake & 7 \\
\hline
\end{tabular}


Table 28. Lake and pond sampling sites in Rocky Mountain National Park, Colorado._Continued

[no., number; identification numbers and station names from USEPA STORET and USGS NWIS]

\begin{tabular}{|c|c|c|c|c|c|c|c|}
\hline $\begin{array}{l}\text { Site no. } \\
\text { (fig. 24) }\end{array}$ & Identification number & Station name & Latitude & Longitude & $\begin{array}{c}\text { Elevation } \\
\text { (meters) }\end{array}$ & Type & $\begin{array}{c}\text { No. } \\
\text { samples }\end{array}$ \\
\hline S99 & 4E1-054 & Fourth Lake & $40^{\circ} 13^{\prime} 18^{\prime \prime}$ & $105^{\circ} 41^{\prime} 09^{\prime \prime}$ & 3,166 & Lake & 9 \\
\hline S100 & 493853118011201 & Gaskil Pond & $40^{\circ} 19^{\prime} 58^{\prime \prime}$ & $105^{\circ} 52^{\prime} 00^{\prime \prime}$ & 2,682 & Pond & 4 \\
\hline S101 & 401827105503600 & Green Mountain Trail & $40^{\circ} 18^{\prime} 27^{\prime \prime}$ & $105^{\circ} 50^{\prime} 38^{\prime \prime}$ & 2,664 & Pond & 1 \\
\hline S102 & 401829105492100 & Green Mountain Trailhead Pond & $40^{\circ} 18^{\prime} 29^{\prime \prime}$ & $105^{\circ} 49^{\prime} 23^{\prime \prime}$ & 2,855 & Pond & 1 \\
\hline S103 & 401830105484900 & Green Mtn. Trail Pond \#3 & $40^{\circ} 18^{\prime} 29^{\prime \prime}$ & $105^{\circ} 48^{\prime} 50^{\prime \prime}$ & 2,871 & Pond & 1 \\
\hline S104 & 402029105453900 & Haynach Lake \#5 & $40^{\circ} 20^{\prime} 28^{\prime \prime}$ & $105^{\circ} 45^{\prime} 42^{\prime \prime}$ & 3,350 & Lake & 1 \\
\hline S105 & 402040105454100 & Haynach Lakes \#2-4 & $40^{\circ} 20^{\prime} 41^{\prime \prime}$ & $105^{\circ} 45^{\prime} 43^{\prime \prime}$ & 3,365 & Lake & 1 \\
\hline S106 & 4E1-018 & Haynach Lakes (Northwest) & $40^{\circ} 20^{\prime} 47^{\prime \prime}$ & $105^{\circ} 45^{\prime} 57^{\prime \prime}$ & 3,373 & Lake & 3 \\
\hline S107 & 402445105491400 & Lake Irene & $40^{\circ} 24^{\prime} 45^{\prime \prime}$ & $105^{\circ} 49^{\prime} 16^{\prime \prime}$ & 3,231 & Lake & 9 \\
\hline S108 & 4E1-027 & Lake Nanita & $40^{\circ} 15^{\prime} 23^{\prime \prime}$ & $105^{\circ} 43^{\prime} 02^{\prime \prime}$ & 3,288 & Lake & 6 \\
\hline S109 & 401552105433300 & Lake Nokoni & $40^{\circ} 15^{\prime} 50^{\prime \prime}$ & $105^{\circ} 43^{\prime} 42^{\prime \prime}$ & 3,286 & Lake & 6 \\
\hline S110 & 401601105423800 & Lake Solitude & $40^{\circ} 16^{\prime} 01^{\prime \prime}$ & $105^{\circ} 42^{\prime} 40^{\prime \prime}$ & 2,969 & Lake & 1 \\
\hline S111 & 4E1-028 & Lake Verna & $40^{\circ} 13^{\prime} 35^{\prime \prime}$ & $105^{\circ} 42^{\prime} 30^{\prime \prime}$ & 3,105 & Lake & 10 \\
\hline S112 & 401910105481800 & Lilly Pond at Big Meadows & $40^{\circ} 19^{\prime} 10^{\prime \prime}$ & $105^{\circ} 48^{\prime} 20^{\prime \prime}$ & 2,871 & Pond & 1 \\
\hline S113 & 401324105435400 & Lone Pine Lake & $40^{\circ} 13^{\prime} 58^{\prime \prime}$ & $105^{\circ} 43^{\prime} 53^{\prime \prime}$ & 3,078 & Lake & 7 \\
\hline S114 & 402221105513400 & Never Summer Ranch & $40^{\circ} 22^{\prime} 21^{\prime \prime}$ & $105^{\circ} 51^{\prime} 36^{\prime \prime}$ & 2,707 & Pond & 3 \\
\hline S115 & 401713105443100 & Ptarmigan Pond & $40^{\circ} 17^{\prime} 11^{\prime \prime}$ & $105^{\circ} 44^{\prime} 32^{\prime \prime}$ & 2,816 & Pond & 1 \\
\hline S116 & 4E1-058 & Snowdrift Lake & $40^{\circ} 18^{\prime} 25^{\prime \prime}$ & $105^{\circ} 44^{\prime} 06^{\prime \prime}$ & 3,373 & Lake & 3 \\
\hline S117 & 401246105414900 & Spirit Lake & $40^{\circ} 13^{\prime} 25^{\prime \prime}$ & $105^{\circ} 41^{\prime} 45^{\prime \prime}$ & 3,127 & Lake & 7 \\
\hline S118 & 402251105511200 & Timber Creek Campground & $40^{\circ} 22^{\prime} 51^{\prime \prime}$ & $105^{\circ} 51^{\prime} 14^{\prime \prime}$ & 2,722 & Pond & 16 \\
\hline S119 & $4 \mathrm{E} 1-057$ & Timber Lake & $40^{\circ} 22^{\prime} 27^{\prime \prime}$ & $105^{\circ} 47^{\prime} 47^{\prime \prime}$ & 3,373 & Lake & 10 \\
\hline S120 & 402228105481800 & Timber Lake Pond \#4 & $40^{\circ} 22^{\prime} 26^{\prime \prime}$ & $105^{\circ} 48^{\prime} 17^{\prime \prime}$ & 3,322 & Pond & 1 \\
\hline S121 & 402224105474700 & Timber Lake Ponds 2 and 3 & $40^{\circ} 22^{\prime} 22^{\prime \prime}$ & $105^{\circ} 47^{\prime} 48^{\prime \prime}$ & 3,383 & Pond & 1 \\
\hline S122 & 401911105481701 & Toad Pond at Benchmark & $40^{\circ} 19^{\prime} 11^{\prime \prime}$ & $105^{\circ} 48^{\prime} 19^{\prime \prime}$ & 2,880 & Pond & 1 \\
\hline
\end{tabular}

the 122 lake and pond sites (table 28, fig. 24), there were 1,515 samples collected from 1974 to 2004 with more than 25,000 individual results, most of which were field properties (26 percent), and major-constituent (40 percent) and nutrient (16 percent) analyses. The types of analyses and numbers of lake and pond samples collected during the period of record are summarized in table 29. Most of the sites (56 percent) were sampled 1 to 3 times during the period of record, but 11 sites were sampled more than 20 times each (table 27). Nearly one-half of the 1,515 samples were collected at sites in LVWS for ecological research including Andrews Tarn (S25), Embryo Pond (S36), Lake of Glass (S61), Sky Pond (S78), and The Loch (S83). Lakes at other locations in the park were sampled as part of studies by the USGS, NPS, U.S. Fish and Wildlife Service, and USEPA, some of which are mentioned below. The USEPA sampled 23 lakes in 1985 as part of the Western Lake Survey (Landers and others, 1987). The USGS resampled these lakes in 1999, in addition to 24 others, as part of a survey of lakes in high-elevation National Parks (Clow and others, 2002; Clow and others, 2003). The U.S. Fish and Wildlife Service sampled 12 lakes in 1982 as part of an acidic deposition study (Gibson and others, 1983) that were resampled in 1997 as part of a USGS research project (J. Baron, U.S. Geological Survey, written commun., 2005). The LVWS research project monitored four lake sites (S37, S56, S58, S59) outside of LVWS periodically from 1981-2003 (http:// www.nrel.colostate.edu/projects/lvws/, accessed November 2006) and conducted a synoptic survey of 17 lakes in 1998 (J. Baron, U.S. Geological Survey, written commun., 2005). Several small ponds in wetland areas were sampled during 2001 through 2004 as part of the USGS Amphibian Research and Monitoring Initiative (http://armi.usgs.gov/, accessed November 2006). The USGS sampled 10 lakes during 2002 and 2003 to document the occurrence of organochlorine compounds and pesticides in lake sediments (Mast and others, 2006).

For the 83 spring sites (not shown on fig. 24), there were 739 samples collected from 1991 to 2004 with 11,645 results most of which were field properties (23 percent), and major-constituent (45 percent), nutrient (13 percent), and trace-element ( 7 percent) analyses. All but 12 of the spring sites were in LVWS and more than 75 percent of the 739 samples were collected at three LVWS sites-Andrews Spring 1 (USGS station 401727105400000), Spring 19 (USGS station 401707105394800), and Spring 2N (USGS station 401707105394800).

For the 288 stream sites (not shown on fig. 24), there were 5,597 samples collected from 1955 to 2004 with more than 100,000 individual results, most of which were field 


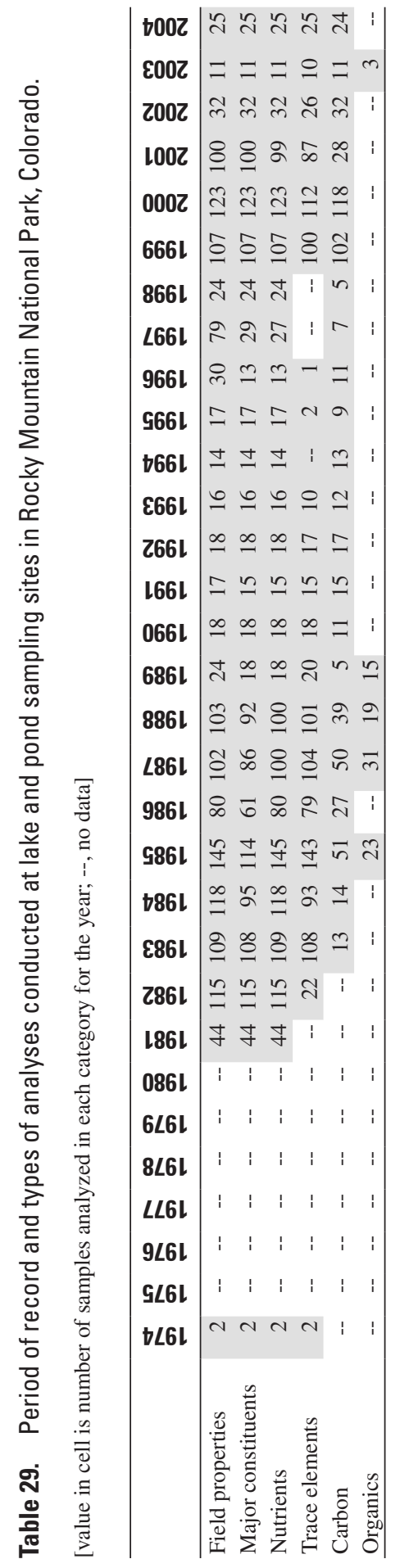

properties (22 percent), and major-constituent (43 percent), nutrient (15 percent), and trace-element ( 5 percent) analyses. Most of the sites (191) were sampled 1 to 3 times during the period of record, but 33 sites were sampled 20 or more times each. More than 60 percent of the 5,625 stream samples were collected in LVWS; the most frequently samples sites were Andrews Creek (USGS station 401723105400000), Icy Brook (USGS station 401707105395000), and The Loch Outlet (USGS station 401733105392404). The water quality of streams in ROMO, and in particular LVWS, has been described extensively in the scientific literature, from which a few of the studies are mentioned here. Baron and Campbell (1997), Campbell and others (1995), and Campbell and others (2000) discuss processes controlling chemical concentrations and fluxes for streams in LVWS. Clow and Sueker (2000) and Sueker and others (2001) examined the relations between basin physical characteristics and solute fluxes and mineral weathering rates for several streams in the park. Mast and others (1995) and Sueker and others (2000) used hydrograph separation techniques based on isotopes of water to determine hydrologic pathways and sources of water during snowmelt. Baron (2006) and Sullivan and others (2005) discuss the effects of sulfur and nitrogen deposition on surface water in LVWS. Mast and others (2005) describe total mercury and methylmercury concentrations and fluxes for selected streams in LVWS. A complete list of publications for LVWS and other surface-water studies in ROMO is available online at http:// www.nrel.colostate.edu/projects/lvws/pages/publications/ publications.htm (accessed November 2006).

Because a large percentage of stream and spring samples were collected in LVWS, and results for many of these are published in the scientific literature, the discussion in this report primarily focuses on the water quality of lakes and ponds in ROMO (table 29). Field properties and major-dissolved constituents for the lakes in ROMO are summarized in table 30. Lake water in ROMO is dilute (median specific conductance $13 \mu \mathrm{S} / \mathrm{cm}$ ) and weakly buffered (median alkalinity $2.9 \mathrm{mg} / \mathrm{L}$ ) because of the slowly weathering crystalline bedrock and rapid hydrologic flushing rates. The dominant anion in surface water was bicarbonate (based on $\mathrm{pH}$ and alkalinity), and the dominant cations were calcium (median $1.5 \mathrm{mg} / \mathrm{L}$ ) and sodium (median $0.50 \mathrm{mg} / \mathrm{L}$ ). The predominance of these constituents is attributed to weathering of plagioclase and trace amounts of calcite in the granite and gneiss (Baron, 1992). Concentrations of sulfate (median $1.4 \mathrm{mg} / \mathrm{L}$ ) and chloride (median $0.13 \mathrm{mg} / \mathrm{L}$ ) also were quite low and similar to concentrations measured in local precipitation (Baron, 1992). Mineral weathering may contribute a small amount of sulfate in some lakes in ROMO although probably not more than 25 percent (Clow and others, 2002). Seasonal variations in selected major-constituent concentrations are shown in figure 26 for Sky Pond (S78) at the surface $(0.2 \mathrm{~m})$ and near the bottom of the lake $(6.0 \mathrm{~m})$. Sky Pond is an alpine lake that is ice covered for 7 months each year and has a maximum depth of $7.3 \mathrm{~m}$ and a surface area of $0.03 \mathrm{~km}^{2}$. The lake thermally stratifies under ice cover; however, wind mixing generally prevents 
Table 30. Summary of selected water-quality data for lake and pond sampling sites in Rocky Mountain National Park, Colorado, 1981 to 2004.

[no. number; chronic aquatic-life (and water-supply) standards from Colorado Department of Public Health and Environment (2006); <, less than; $>$, greater than; --, not reported; ${ }^{\circ} \mathrm{C}$, degrees Celsius; $\mathrm{mg} / \mathrm{L}$, milligrams per liter; $\mu \mathrm{S} / \mathrm{cm}$, microsiemens per centimeter at $25^{\circ} \mathrm{C} ; \mathrm{CaCO}_{3}$, calcium carbonate; N, nitrogen; P, phosphorous; $\mu \mathrm{g} / \mathrm{L}$, micrograms per liter]

\begin{tabular}{|c|c|c|c|c|c|c|}
\hline Constituent or property & No. sites & $\begin{array}{l}\text { No. samples } \\
\text { (no. censored) }\end{array}$ & $\begin{array}{l}\text { Minimum } \\
\text { value }\end{array}$ & $\begin{array}{l}\text { Median } \\
\text { value }\end{array}$ & $\begin{array}{l}\text { Maximum } \\
\text { value }\end{array}$ & $\begin{array}{l}\text { Aquatic-life } \\
\text { (water-supply) } \\
\text { standard }\end{array}$ \\
\hline \multicolumn{7}{|c|}{ Field properties } \\
\hline Temperature, water $\left({ }^{\circ} \mathrm{C}\right)$ & 98 & 1,028 & -0.2 & 6.0 & 33 & $<20$ \\
\hline Oxygen, dissolved (mg/L) & 13 & 51 & .2 & 7.1 & 9.2 & $>6.0$ \\
\hline $\mathrm{pH}$ (standard units) & 112 & 1,328 & 5.45 & 6.61 & 9.40 & $6.5-9.0(5.0-9.0)$ \\
\hline Specific conductance $(\mu \mathrm{S} / \mathrm{cm})$ & 101 & 1,305 & 1.0 & 13 & 773 & -- \\
\hline \multicolumn{7}{|c|}{ Major constituents } \\
\hline Alkalinity $\left(\mathrm{mg} / \mathrm{L}\right.$ as $\mathrm{CaCO}_{3}$ ) & 105 & 1,134 & 0.10 & 2.9 & 75 & -- \\
\hline Calcium, dissolved (mg/L) & 109 & 1,227 & .09 & 1.5 & 142 & -- \\
\hline Chloride, dissolved (mg/L) & 112 & $1,228(7)$ & $<.01$ & .13 & 17 & $\left({ }^{\mathrm{a} 250)}\right.$ \\
\hline Fluoride, dissolved (mg/L) & 61 & $683(31)$ & $<.01$ & .098 & 1.5 & -- \\
\hline Magnesium, dissolved (mg/L) & 109 & 1,247 & .039 & .21 & 15 & -- \\
\hline Potassium, dissolved (mg/L) & 109 & 1,250 & .02 & .17 & 17 & -- \\
\hline Silica, dissolved (mg/L) & 106 & $1,099(3)$ & $<.01$ & 1.7 & 27 & -- \\
\hline Sodium, dissolved (mg/L) & 109 & 1,248 & .11 & .50 & 18 & -- \\
\hline Sulfate, dissolved (mg/L) & 109 & $1,223(3)$ & $<.01$ & 1.4 & 267 & $\left({ }^{2} 250\right)$ \\
\hline \multicolumn{7}{|c|}{ Nutrients and organic carbon } \\
\hline Ammonia, dissolved (mg/L as $\mathrm{N}$ ) & 111 & $433(219)$ & $<0.002$ & $<0.007$ & 11.8 & 0.02 \\
\hline Nitrate, dissolved (mg/L as $\mathrm{N}$ ) & 113 & $1,275(132)$ & b.002 & .16 & 21 & (10) \\
\hline Nitrite, dissolved (mg/L as N) & 9 & $480(152)$ & $<.01$ & .01 & .05 & $.05(1.0)$ \\
\hline Orthophosphate, dissolved (mg/L as P) & 50 & $851(424)$ & $<.001$ & .003 & .036 & -- \\
\hline Phosphorus, dissolved (mg/L as P) & 30 & $59(18)$ & $<.005$ & .01 & .16 & -- \\
\hline Organic carbon, dissolved (mg/L) & 93 & $614(2)$ & $<.1$ & 1.2 & 76 & -- \\
\hline \multicolumn{7}{|c|}{ Trace elements } \\
\hline Aluminum, dissolved ( $\mu \mathrm{g} / \mathrm{L})$ & 8 & $443(0)$ & 0.02 & 20 & 160 & -- \\
\hline Iron, dissolved ( $\mu \mathrm{g} / \mathrm{L})$ & 20 & $644(4)$ & $<2$ & 32 & 800 & ${ }^{\mathrm{c}} 1,000\left({ }^{\mathrm{a}} 300\right)$ \\
\hline Manganese, dissolved ( $\mu \mathrm{g} / \mathrm{L})$ & 38 & $595(25)$ & $<1$ & 3 & 520 & $50\left({ }^{a} 50\right)$ \\
\hline Strontium, dissolved ( $\mu \mathrm{g} / \mathrm{L})$ & 96 & 363 & 2 & 7 & 442 & -- \\
\hline
\end{tabular}

${ }^{\text {a}}$ Secondary standard based on aesthetic properties such as taste, odor, and staining.

${ }^{b}$ Minimum reported value less than minimum censored value.

cTotal recoverable concentration.

stratification during the open water season (Baron, 1992). Concentrations of weathering-derived constituents (calcium and alkalinity) decrease rapidly in spring and reach a minimum in mid summer as the lake is diluted by large inflows of dilute snowmelt. Concentrations increase steadily through the fall and early winter as snowmelt declines and shallow ground water contributes a greater percentage of flow to lakes and streams. Some of the increase in concentrations during early winter also may be caused by ice formation, which converts more than one-half of the lake volume of water (Baron, 1992). Chloride, which largely is derived from atmospheric sources, showed a slightly different seasonal pattern. Concentrations decrease in early summer due to snowmelt dilution then began to increase in late summer and early fall because of evaporation and ground-water inputs. Once the lake became ice-covered, concentrations remained remarkably constant indicating 

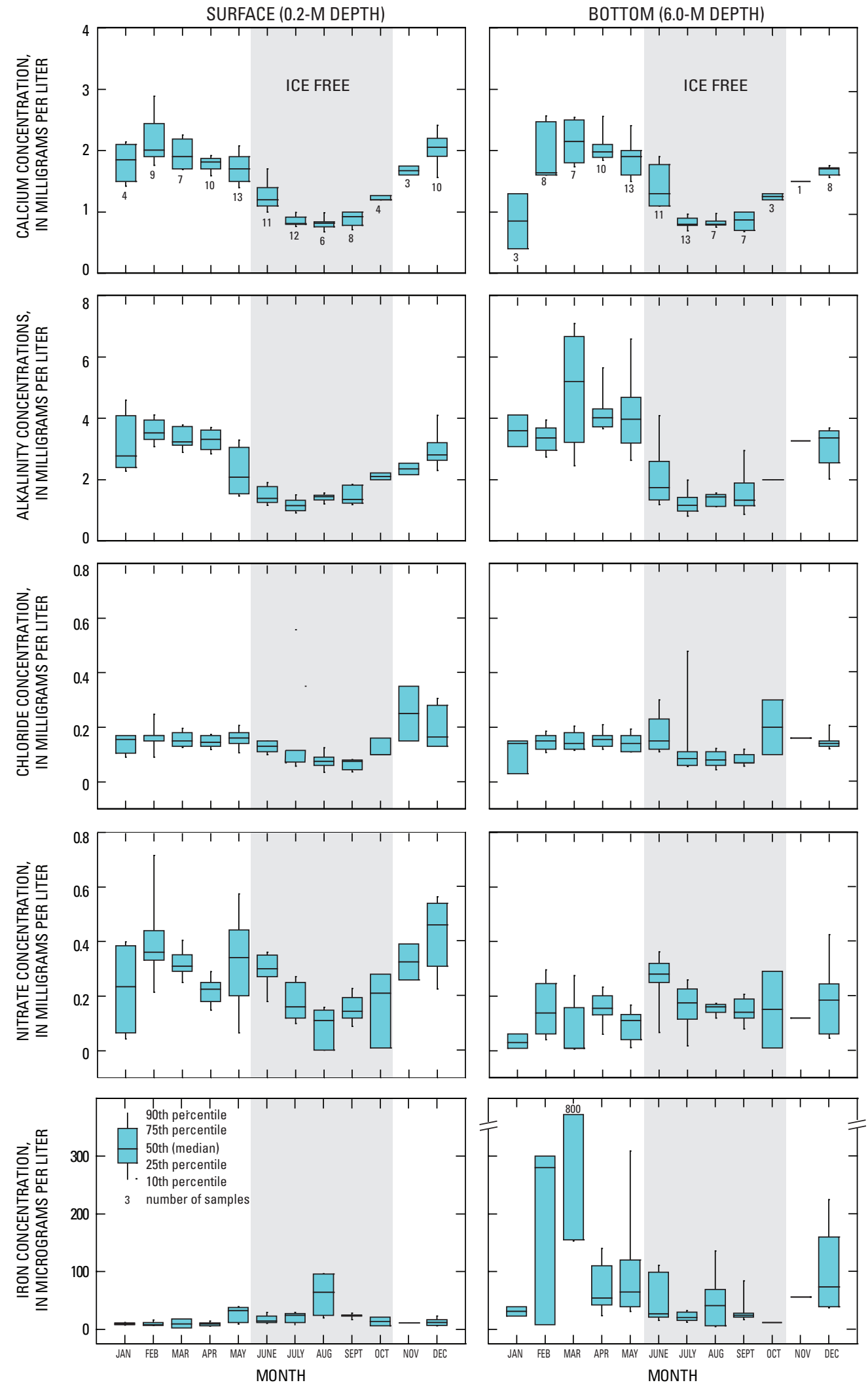

Figure 26. Seasonal variation in selected constituent concentrations in water samples from the surface and bottom of Sky Pond (S78) in Rocky Mountain National Park, Colorado, 1982-2004. 
chloride was minimally affected by in-lake biological and geochemical processes. Concentrations of major constituents were similar between the two sampling depths $(0.2$ and 6.0 $\mathrm{m}$ ) except for alkalinity, which was elevated in the subsurface during early spring. This likely was caused by dissolved oxygen depletion in water at the bottom of the lake during winter (Baron, 1992), Low dissolved oxygen concentrations in lake water allow buildup of bicarbonate, iron, and manganese that are generated by anaerobic respiration reactions in the lake sediments.

Spatial patterns in major constituents are illustrated by comparing lakes on different sides of the Continental Divide (fig. 27). Probabilities (p-values) shown on the figure are for statistical comparison of east- and west-side lakes using the Wilcoxon signed-rank test. Of the 86 lakes used for the comparison (small wetland ponds were excluded), 63 were on the east side of he divide and 23 were on the west side. The greater number of east-side lakes largely reflects the geographic distribution of lakes in the park. East-side lakes span a wider range in elevation than west-side lakes; however, there was no statistical difference in the two groups. Calcium concentrations indicated very little difference between the two groups of lakes, indicating weathering processes are fairly uniform. Sulfate concentrations, which primarily are derived from atmospheric sources, also had little difference, indicating rates of sulfur deposition are not substantially different on the two sides of the Continental Divide. None of the other major constituents indicated statistically significant differences in concentrations between east and west-side lakes.

Nutrients measured in lake-water samples from ROMO include ammonia, nitrate, nitrite, orthophosphate, and dissolved phosphorus (table 30). Ammonia concentrations ranged from less than .002 to $11.8 \mathrm{mg} / \mathrm{L}$ and 95 percent of samples had concentrations less than $0.1 \mathrm{mg} / \mathrm{L}$. The highest ammonium concentrations ( 0.3 to $11.8 \mathrm{mg} / \mathrm{L}$ ) were collected at Kettle Tarn (S55), which is a shallow wetland pond that provides important amphibian habitat. Elevated concentrations were measured following a drought in 2002 and are attributed to the development of anoxic conditions that caused mineralization of organic matter at the sediment/water interface (D. Campbell, U.S. Geological Survey, oral commun., 2006). Samples collected at Kettle Tarn in 2004 also had very high nitrate concentrations (more than $15 \mathrm{mg} / \mathrm{L}$ ), which probably resulted from conversion of ammonia to nitrate (nitrification) once oxidizing conditions were restored in the pond.

Nitrate concentrations for other sites in ROMO ranged from 0.002 to $0.81 \mathrm{mg} / \mathrm{L}$, some of which were the largest reported for high-elevation lakes in the Rocky Mountains (Burns, 2004). As mentioned previously, elevated nitrate in surface water is attributed to atmospheric deposition of nitrogen, which is released to the atmosphere by fossil fuel combustion and agricultural activities. When the availability of inorganic nitrogen exceeds biological demand, the ecosystem reaches nitrogen saturation and nitrate begins leaching to surface waters (Williams and others, 1996). The degree to which nitrogen saturation is occurring in ROMO is illustrated by temporal and spatial patterns in surface-water nitrate concentrations. Concentrations are lowest in summer when biological demand is high, then increase rapidly through fall and early winter once the growing season ends (fig. 26). Concentrations peak again in late April and early May because of elution from the snowpack and flushing of soils during the early stages of snowmelt (Campbell and others, 1995). This seasonal pattern is typical of watersheds in the northeastern U.S. that receive much higher loadings of atmospheric nitrogen (Burns, 2004). Nitrate concentrations in east-side lakes were found to be significantly higher than west-side lakes indicating ecosystems on the east side may be at a more advanced stage of nitrogen saturation
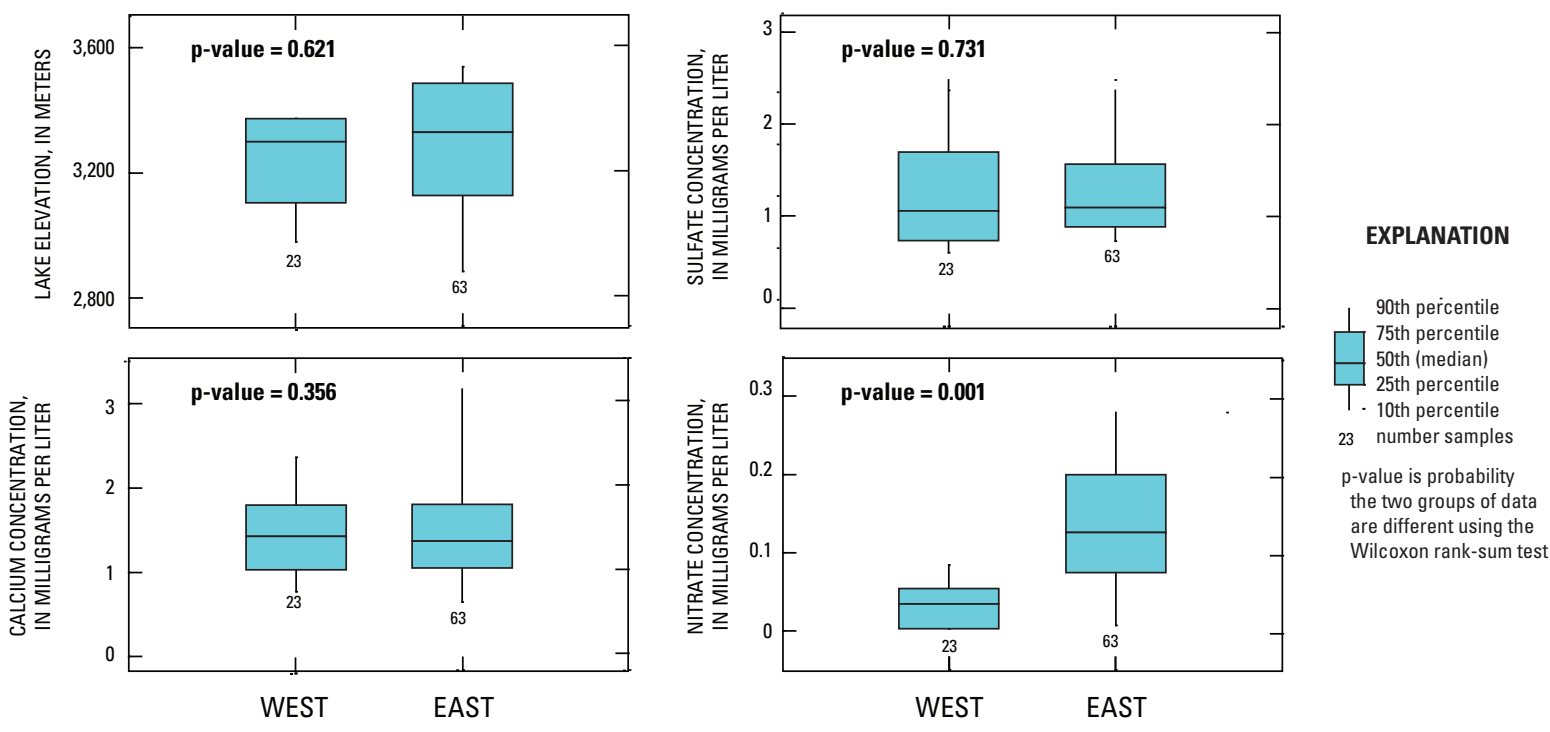

Figure 27. Comparison of water-quality data for selected lakes on the east and west sides of the Continental Divide in Rocky Mountain National Park, Colorado. 
(fig. 27). Some studies indicate this may reflect higher deposition rates east of the Continental Divide because of proximity to nitrogen source areas (Baron and others, 2000). Other studies have shown that variations in vegetation and soil cover, and the distribution of talus deposits explain most of the spatial variation in nitrate concentrations (Clow and Sueker, 2000; Sueker and others, 2001). In contrast to nitrogen, phosphorus species showed much lower concentrations. More than 99 percent of orthophosphate concentrations were below $0.01 \mathrm{mg} / \mathrm{L}$, and 90 percent of total phosphorous concentrations were below $0.05 \mathrm{mg} / \mathrm{L}$.

Concentrations for only four trace elements were reported for lakes and ponds sampled in ROMO: aluminum, iron, manganese, and strontium (table 30). Aluminum concentrations ranged from 0.02 to $160 \mu \mathrm{g} / \mathrm{L}$ and concentrations generally were higher during the snow-free season particularly during snowmelt. Higher aluminum concentrations during runoff could be related to increased suspended sediment in surface water or flushing of organically complexed aluminum from soils. Iron concentrations ranged from less than 2 to $800 \mu \mathrm{g} / \mathrm{L}$ and manganese ranged from less than 1 to $520 \mu \mathrm{g} / \mathrm{L}$. The majority of iron and manganese concentrations above $50 \mu \mathrm{g} / \mathrm{L}$ were reported for bottom-water samples collected from lakes during late winter and early spring (fig. 26). This occurrence is caused by depletion of dissolved oxygen at the bottom of the lake during winter stratification, which allows buildup of iron and manganese generated by anaerobic respiration reactions in the lake sediments. Strontium concentrations ranged from 2 to $442 \mu \mathrm{g} / \mathrm{L}$ and were highest in samples collected from shallow pond sites. These samples also had fairly high concentrations of major constituents, including chloride, indicating they may have been elevated by evaporative processes in the ponds and surrounding wetlands. Strontium also was found to be strongly correlated with calcium, indicating it is derived from the same source. Using strontium isotopes as a tracer, Clow and others (1997) determined that weathering of plagioclase, calcite, and eolian dust was the main process controlling calcium (and strontium) concentrations in surface water in LVWS.

Sufficient data to test for trends in water quality with time were available for one lake site and three stream sites, all of which are in LVWS (table 31, fig. 24). All sites were tested for trends in discharge and major-constituent and nitrate concentrations, except for Sky Pond, which was not gaged for streamflow. Sky Pond and The Loch, which had the longest records, were tested from 1984 to 2004, and Andrews Creek and Icy Brook were tested from 1992 to 2004. Statistically significant ( $\mathrm{p}$-value $\leq 0.01$ ) upward trends were detected for several constituents although none of the sites had trends in discharge. From 1984 to 2004, Sky Pond and The Loch had upward trends in unadjusted alkalinity and sodium, and The Loch also showed upward trends in unadjusted calcium, magnesium, and nitrate concentrations that were similar in magnitude to trends detected using flow-adjusted concentrations. Andrews Creek had significant trends in calcium, magnesium, potassium, sodium, sulfate, and nitrate that were similar between unadjusted and flow-adjusted concentrations from 1992 to
2004. Icy Brook had similar trends during the same period in unadjusted calcium, magnesium, and sodium concentrations that were not significant in the flow-adjusted concentrations.

Three of the four sites had statistically significant increases in surface-water nitrate concentrations, which could reflect long-term increases in atmospheric deposition of nitrate and ammonia. Baron (2006) determined that total nitrogen deposition in LVWS has increased by 2.5 percent per year since 1984 because of increases in nitrogen emissions from fossil fuel and agricultural sources. The magnitude of the deposition trend was similar to nitrate in The Loch, which increased about 3 percent per year since 1984. Increases in strong acid anions (nitrate and sulfate) because of acidic deposition often are offset by declines in the acid neutralizing capacity (or alkalinity) of streams (Galloway and others, 1983). Interestingly, none of the streams showed declines in alkalinity and The Loch actually had a slight upward trend from 1984 to 2004 . The increase in stream-water nitrate appeared to be associated with increases in cation concentrations, particularly calcium and magnesium This could reflect leaching of base cations from the soil by acidic compounds (sulfuric and nitric acids), which is a common watershed response to acidic precipitation (Galloway and others, 1983).

An alternative explanation for the observed trends in stream-water quality could be patterns in climate, particularly precipitation amount. Although there were no detected trends in discharge, only daily discharge associated with each waterquality sample was tested, which is probably insufficient for detecting long-term trends in streamflow. From 1995 to 2004, annual precipitation amount at the NADP station in LVWS showed a statistically significant $(\mathrm{p}=.010)$ downward trend, reaching a minimum in 2002 during a regional drought (fig. 28). A time-series plot of calcium for The Loch shows that most of the increase in concentration occurred over the last decade, which is coincident with the decline in annual precipitation (fig. 28). Because seasonality strongly affects chemical concentrations in most high-elevation streams, the seasonal patterns of concentrations may differ from year to year, depending on the amounts of snowfall and summer rainfall as well as other climate variables, such as temperature (Campbell and others, 1995). To some degree, both acidic deposition and precipitation amount are likely driving water-quality trends, and continued monitoring will be crucial for sorting out the relative importance of these processes.

Comparison to Water-Quality Standards. Water-quality use classifications and standards for all stream segments in Colorado are established by the CDPHE (Colorado Department of Public Health and Environment, 2003). All tributaries, wetlands, lakes, and reservoirs in ROMO are included in segment 1 of the Upper Colorado River Basin, segment 1 of the Saint Vrain Creek, segment 1 of the Big Thompson River, and segment 1 of the Cache La Poudre River (Colorado Department of Public Health and Environment, 2006). The classified uses for these segments are cold-water aquatic life class 1 , recreation 1a, agriculture, and water supply. None of these segments were listed in 2006 as impaired by the 
Table 31. Results of the seasonal Kendall test for trends in streamflow and unadjusted and flow-adjusted constituent concentrations for Rocky Mountain National Park, Colorado.

[--, not calculated or flow model not statistically significant at $\mathrm{p} \leq 0.1 ; \mathrm{ft}^{3} / \mathrm{s} / \mathrm{yr}, \mathrm{cubic}$ feet per second per year; mg/L/yr, milligrams per liter per year; ; N, nitrogen; trends in bold are significant at $\mathrm{p} \leq 0.01]$

\begin{tabular}{|c|c|c|c|c|c|}
\hline \multirow[b]{2}{*}{ Parameter } & \multicolumn{2}{|c|}{ Unadjusted } & \multicolumn{2}{|c|}{ Flow-adjusted } & \multirow{2}{*}{$\begin{array}{l}\text { Period of } \\
\text { record }\end{array}$} \\
\hline & trend & p-value & trend & p-value & \\
\hline \multicolumn{6}{|c|}{ Sky Pond (S78) } \\
\hline pH (standard units/yr) & 0.018 & 0.057 & -- & -- & 1984-2004 \\
\hline Alkalinity (mg/L/yr) & .026 & .007 & -- & -- & 1984-2004 \\
\hline Calcium (mg/L/yr) & .016 & .051 & -- & -- & 1984-2004 \\
\hline Chloride (mg/L/yr) & $<.001$ & .152 & -- & -- & 1984-2004 \\
\hline Magnesium (mg/L/yr) & .003 & .027 & -- & -- & 1984-2004 \\
\hline Potassium (mg/L/yr) & $<.001$ & .951 & -- & -- & 1984-2004 \\
\hline Silica (mg/L/yr) & .022 & .056 & -- & -- & 1984-2004 \\
\hline Sodium (mg/L/yr) & .010 & .003 & -- & -- & 1984-2004 \\
\hline Sulfate (mg/L/yr) & .027 & .047 & -- & -- & 1984-2004 \\
\hline Nitrate (mg as N/L/yr) & .006 & .030 & -- & -- & 1984-2004 \\
\hline \multicolumn{6}{|c|}{ The Loch (S83) } \\
\hline Streamflow $\left(\mathrm{ft}^{3} / \mathrm{s} / \mathrm{yr}\right)$ & $<0.01$ & 0.751 & -- & -- & 1984-2004 \\
\hline pH (standard units/yr) & .015 & .035 & -- & -- & 1984-2004 \\
\hline Alkalinity (mg/L/yr) & .025 & .010 & 0.021 & 0.004 & 1984-2004 \\
\hline Calcium (mg/L/yr) & .024 & .001 & .022 & .004 & 1984-2004 \\
\hline Chloride (mg/L/yr) & .003 & .015 & .002 & .037 & 1984-2004 \\
\hline Magnesium (mg/L/yr) & .005 & .001 & .004 & .001 & 1984-2004 \\
\hline Potassium (mg/L/yr) & .002 & .095 & .001 & .171 & 1984-2004 \\
\hline Silica $(\mathrm{mg} / \mathrm{L} / \mathrm{yr})$ & .021 & .059 & .021 & .078 & 1984-2004 \\
\hline Sodium (mg/L/yr) & .013 & .001 & .011 & .001 & 1984-2004 \\
\hline Sulfate (mg/L/yr) & .032 & .032 & .031 & .020 & 1984-2004 \\
\hline Nitrate (mg as N/L/yr) & .008 & .001 & -- & -- & 1984-2004 \\
\hline \multicolumn{6}{|c|}{ Andrews Creek (USGS station 401723105400000) } \\
\hline Streamflow $\left(\mathrm{ft}^{3} / \mathrm{s} / \mathrm{yr}\right)$ & -0.003 & 0.610 & -- & -- & $1992-2004$ \\
\hline $\mathrm{pH}$ (standard units/yr) & -.011 & .094 & -0.012 & 0.050 & 1992-2004 \\
\hline Alkalinity (mg/L/yr) & .005 & .349 & .003 & .644 & $1992-2004$ \\
\hline Calcium (mg/L/yr) & .043 & .001 & .045 & .003 & $1992-2004$ \\
\hline Chloride (mg/L/yr) & .003 & .049 & .003 & .043 & 1992-2004 \\
\hline Magnesium (mg/L/yr) & .007 & .003 & .007 & .004 & 1992-2004 \\
\hline Potassium (mg/L/yr) & .004 & .009 & .004 & .012 & 1992-2004 \\
\hline Silica (mg/L/yr) & .034 & .013 & .026 & .039 & 1992-2004 \\
\hline Sodium (mg/L/yr) & .013 & .011 & .012 & .008 & 1992-2004 \\
\hline Sulfate (mg/L/yr) & .063 & .009 & .064 & .003 & 1992-2004 \\
\hline Nitrate (mg as N/L/yr) & .023 & .001 & .024 & .001 & 1992-2004 \\
\hline \multicolumn{6}{|c|}{ Icy Brook (USGS station 401707105395000) } \\
\hline Streamflow $\left(\mathrm{ft}^{3} / \mathrm{s} / \mathrm{yr}\right)$ & $<0.01$ & 0.589 & -- & -- & 1992-2004 \\
\hline $\mathrm{pH}$ (standard units/yr) & -.010 & .229 & -- & -- & 1992-2004 \\
\hline Alkalinity (mg/L/yr) & .030 & .044 & -- & -- & 1992-2004 \\
\hline Calcium (mg/L/yr) & .045 & .004 & 0.047 & 0.018 & 1992-2004 \\
\hline Chloride (mg/L/yr) & .002 & .219 & -- & -- & 1992-2004 \\
\hline Magnesium (mg/L/yr) & .007 & .006 & .007 & .024 & 1992-2004 \\
\hline Potassium (mg/L/yr) & .003 & .125 & -- & -- & 1992-2004 \\
\hline Silica (mg/L/yr) & -.005 & .643 & -- & -- & 1992-2004 \\
\hline Sodium (mg/L/yr) & .015 & .002 & .015 & .021 & 1992-2004 \\
\hline Sulfate (mg/L/yr) & .061 & .030 & .074 & .070 & 1992-2004 \\
\hline Nitrate (mg as N/L/yr) & .016 & .017 & -- & -- & 1992-2004 \\
\hline
\end{tabular}




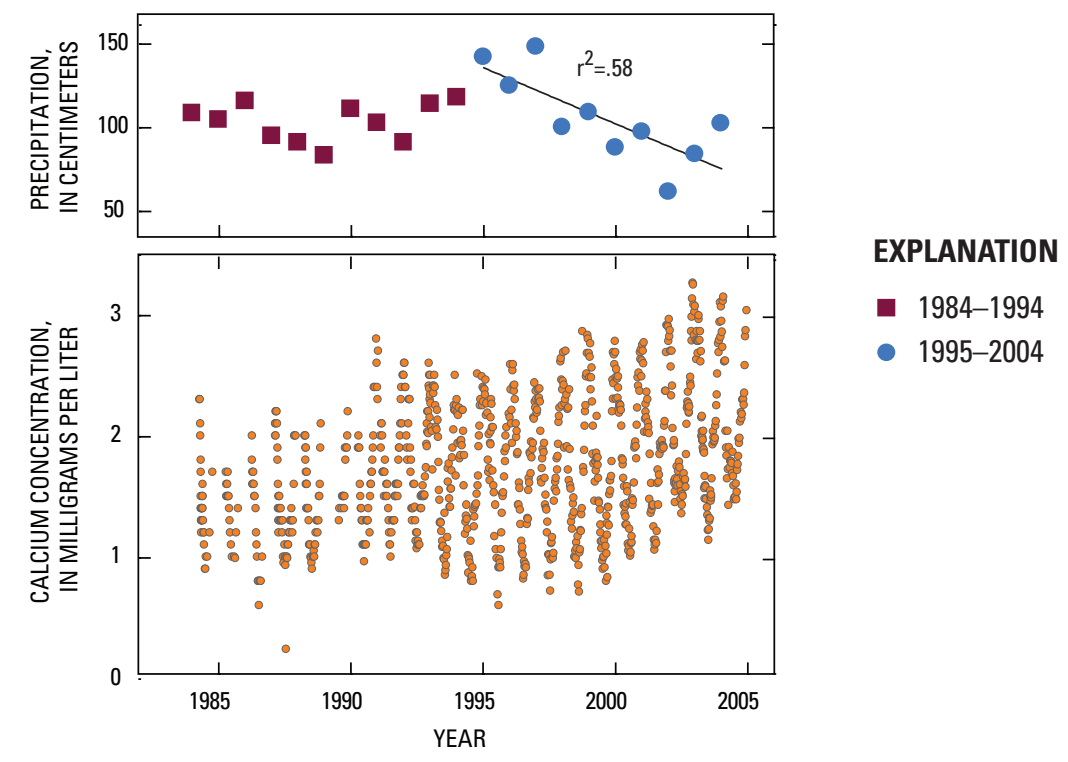

Figure 28. Temporal trends in annual precipitation at Loch Vale NADP station and calcium concentrations at The Loch (S33) in Rocky Mountain National Park, Colorado.

CDPHE (http://www.cdphe.state.co.us/op/wqcc/SpecialTopics /303(d)/303dtmdlpro.html, accessed November 2006). Overall, the water quality of the park is excellent; however, there were several samples that exceeded the aquatic-life standards for $\mathrm{pH}$, ammonia, nitrate, and manganese (table 30). About 30 percent of samples had $\mathrm{pH}$ values that were less than the lower $\mathrm{pH}$ limit of 6.5. These low $\mathrm{pH}$ values reflect the naturally low alkalinity of lakes in the park. Several samples exceeded the standard for ammonium and nitrate, all of which were collected at the Kettle Tarn site. As mentioned previously, the unusually high nutrient concentrations at this site likely are related to development of anoxic conditions in the pond that occurred during the 2002 drought. Subsurface water samples from a few high-elevation lakes exceeded the manganese standard of $50 \mu \mathrm{g} / \mathrm{L}$ likely due to depletion of dissolved oxygen at the bottom of the lake during winter stratification.

There is a growing body of scientific data that indicates atmospheric deposition of nitrogen compounds has caused increases in nitrate concentrations of surface water and impairment of aquatic and terrestrial ecosystems. Current (2007) levels of nitrate in surface water do not exceed numeric standards that have been adopted for the classified uses; however, the standards do not directly apply to terrestrial or aquatic effects, other than cold-water fisheries. All surface water within the park boundary has "Outstanding Natural Waters" designation, which provides the highest level of regulatory protection provided under the Clean Water Act and Colorado regulations (Colorado Department of Public Health and Environment, 2006). Outstanding waters are protected under the Antidegradation Rule, which states that these waters shall be maintained and protected at their existing quality. The traditional program implementing this protection, however, regulates pollutants coming from point source discharges. Atmospheric deposition is not defined as point-source discharge, and the State of Colorado has not previously applied the Antidegredation Rule to non-point atmospheric sources (http://www2.nature.nps.gov/air/Pubs/ pdf/2005romoPolicyOptions.pdf, accessed November 2006).

\section{Ground-Water Quality}

Water-quality data were collected at 30 ground-water wells within the boundaries of ROMO (table 32). For these 30 wells, there are 76 samples with 1,121 individual data results, most of which are field properties (20 percent), and major-constituent (51 percent) and nutrient (13 percent) analyses. Seven of the wells (G1-G7) were sampled once each by the USGS before 1980, although the purpose for the sampling could not be determined. These wells are located at relatively low elevations (below 2,800 m) in the vicinity of park facilities, and probably are supply wells. The remaining 23 wells are located in the LVWS and were sampled by the USGS as part of the Water, Energy, and Biogeochemical Budgets Program. All the wells in LVWS are shallow (0.3 to $1.8 \mathrm{~m}$ ) observation wells that were installed in high-elevation wetlands (above 3,100 m) situated at the base of talus slopes (Bachman, 1994).

Water-quality summaries for the two groups of wells (LVWS and supply wells) in ROMO are presented in table 33. Ground water in LVWS is dilute and weakly buffered. Specific conductance ranged from 17 to $50 \mu \mathrm{S} / \mathrm{cm}$, and alkalinity ranged from 3.3 to $22 \mathrm{mg} / \mathrm{L}$. The major cations were calcium and sodium, and the dominant anion was bicarbonate (based on $\mathrm{pH}$ and alkalinity). Low dissolved solids in these wells are attributed to chemically resistant bedrock and short hydrologic residence times that are characteristic of high-elevation areas. Chloride (median $0.14 \mathrm{mg} / \mathrm{L}$ ) and sulfate (median $2.9 \mathrm{mg} / \mathrm{L}$ ) 
Table 32. Ground-water sampling sites in Rocky Mountain National Park, Colorado.

[ --, no data; identification numbers and station names from USGS NWIS]

\begin{tabular}{|c|c|c|c|c|c|c|c|}
\hline $\begin{array}{l}\text { Site no. } \\
\text { (fig. 24) }\end{array}$ & $\begin{array}{c}\text { Identification } \\
\text { number }\end{array}$ & Station name & Latitude & Longitude & $\begin{array}{l}\text { Well depth } \\
\text { (meters) }\end{array}$ & $\begin{array}{l}\text { Period of } \\
\text { record }\end{array}$ & $\begin{array}{c}\text { Number of } \\
\text { samples }\end{array}$ \\
\hline \multicolumn{8}{|c|}{ Supply wells } \\
\hline G1 & 401609105502000 & Harbison Utility Well & $40^{\circ} 16^{\prime} 09^{\prime \prime}$ & $105^{\circ} 50^{\prime} 29^{\prime \prime}$ & -- & 1974 & 1 \\
\hline $\mathrm{G} 2$ & 401621105332500 & SB00407334BBD & $40^{\circ} 16^{\prime} 21^{\prime \prime}$ & $105^{\circ} 33^{\prime} 25^{\prime \prime}$ & -- & 1971 & 1 \\
\hline G3 & 401807105503800 & Onahu Well & $40^{\circ} 18^{\prime} 07^{\prime \prime}$ & $105^{\circ} 50^{\prime} 38^{\prime \prime}$ & -- & 1974 & 1 \\
\hline G4 & 401820105503300 & Green Mountain Well 2 & $40^{\circ} 18^{\prime} 20^{\prime \prime}$ & $105^{\circ} 50^{\prime} 33^{\prime \prime}$ & -- & 1974 & 1 \\
\hline G5 & 402252105511500 & Timber Creek Campground & $40^{\circ} 22^{\prime} 52^{\prime \prime}$ & $105^{\circ} 51^{\prime} 15^{\prime \prime}$ & -- & 1974 & 1 \\
\hline G6 & 402253105510501 & SB00507624ADC & $40^{\circ} 22^{\prime} 53^{\prime \prime}$ & $105^{\circ} 51^{\prime} 05^{\prime \prime}$ & 30 & 1973 & 1 \\
\hline G7 & 402412105353001 & B00507317ADB & $40^{\circ} 24^{\prime} 12^{\prime \prime}$ & $105^{\circ} 35^{\prime} 30^{\prime \prime}$ & 31 & 1965 & 1 \\
\hline \multicolumn{8}{|c|}{ Loch Vale wells } \\
\hline G8 & 401707105394502 & Well A020 & $40^{\circ} 17^{\prime} 07^{\prime \prime}$ & $105^{\circ} 39^{\prime} 45^{\prime \prime}$ & 1.3 & 1994 & 1 \\
\hline G9 & 401707105394503 & Well A030 & $40^{\circ} 17^{\prime} 07^{\prime \prime}$ & $105^{\circ} 39^{\prime} 45^{\prime \prime}$ & 1.2 & 1994 & 1 \\
\hline G10 & 401711105394401 & Well B010 & $40^{\circ} 17^{\prime} 11^{\prime \prime}$ & $105^{\circ} 39^{\prime} 44^{\prime \prime}$ & 1.3 & 1994,2003 & 3 \\
\hline G11 & 401711105394402 & Well B020 & $40^{\circ} 17^{\prime} 11^{\prime \prime}$ & $105^{\circ} 39^{\prime} 44^{\prime \prime}$ & 1.4 & 1994 & 2 \\
\hline G12 & 401711105394403 & Well B030 & $40^{\circ} 17^{\prime} 11^{\prime \prime}$ & $105^{\circ} 39^{\prime} 44^{\prime \prime}$ & 1.3 & 1994 & 2 \\
\hline G13 & 401711105394404 & Well B040 & $40^{\circ} 17^{\prime} 11^{\prime \prime}$ & $105^{\circ} 39^{\prime} 44^{\prime \prime}$ & 1.6 & 1994 & 2 \\
\hline G14 & 401711105394405 & Well B050 & $40^{\circ} 17^{\prime} 11^{\prime \prime}$ & $105^{\circ} 39^{\prime} 44^{\prime \prime}$ & 1.3 & 1994 & 2 \\
\hline G15 & 401713105394601 & Well C010 & $40^{\circ} 17^{\prime} 13^{\prime \prime}$ & $105^{\circ} 39^{\prime} 46^{\prime \prime}$ & 1.3 & 1994-95, 2003 & 14 \\
\hline G16 & 401713105394602 & Well C020 & $40^{\circ} 17^{\prime} 13^{\prime \prime}$ & $105^{\circ} 39^{\prime} 46^{\prime \prime}$ & 1.1 & 1994 & 1 \\
\hline G17 & 401713105394603 & Well C030 & $40^{\circ} 17^{\prime} 13^{\prime \prime}$ & $105^{\circ} 39^{\prime} 46^{\prime \prime}$ & 1.1 & 1994 & 1 \\
\hline G18 & 401713105394604 & Well C040 & $40^{\circ} 17^{\prime} 13^{\prime \prime}$ & $105^{\circ} 39^{\prime} 46^{\prime \prime}$ & 1.0 & 1994 & 1 \\
\hline G19 & 401713105394605 & Well C050 & $40^{\circ} 17^{\prime} 13^{\prime \prime}$ & $105^{\circ} 39^{\prime} 46^{\prime \prime}$ & 1.3 & 1994 & 1 \\
\hline G20 & 401713105394606 & Well C060 & $40^{\circ} 17^{\prime} 13^{\prime \prime}$ & $105^{\circ} 39^{\prime} 46^{\prime \prime}$ & 1.3 & 1994 & 1 \\
\hline G21 & 401713105394607 & Well C070 & $40^{\circ} 17^{\prime} 13^{\prime \prime}$ & $105^{\circ} 39^{\prime} 46^{\prime \prime}$ & 1.0 & 1994 & 1 \\
\hline G22 & 401713105394608 & Well C080 & $40^{\circ} 17^{\prime} 13^{\prime \prime}$ & $105^{\circ} 39^{\prime} 46^{\prime \prime}$ & 1.3 & 1994 & 1 \\
\hline G23 & 401713105394609 & Well C090 & $40^{\circ} 17^{\prime} 13^{\prime \prime}$ & $105^{\circ} 39^{\prime} 46^{\prime \prime}$ & 1.8 & 1994 & 1 \\
\hline $\mathrm{G} 24$ & 401713105394610 & Well C100 & $40^{\circ} 17^{\prime} 13^{\prime \prime}$ & $105^{\circ} 39^{\prime} 46^{\prime \prime}$ & 1.4 & 1994 & 1 \\
\hline G25 & 401713105394611 & Well C110 & $40^{\circ} 17^{\prime} 13^{\prime \prime}$ & $105^{\circ} 39^{\prime} 46^{\prime \prime}$ & 1.5 & 1994 & 1 \\
\hline G26 & 401713105394612 & Well C120 & $40^{\circ} 17^{\prime} 13^{\prime \prime}$ & $105^{\circ} 39^{\prime} 46^{\prime \prime}$ & 1.1 & 1994 & 1 \\
\hline G27 & 401713105394613 & Well C130 & $40^{\circ} 17^{\prime} 13^{\prime \prime}$ & $105^{\circ} 39^{\prime} 46^{\prime \prime}$ & 1.1 & 1994 & 1 \\
\hline G28 & 401723105400014 & Well ASC1 & $40^{\circ} 17^{\prime} 23^{\prime \prime}$ & $105^{\circ} 40^{\prime} 00^{\prime \prime}$ & .9 & 1996-2002 & 15 \\
\hline G29 & 401723105400015 & Well ASC2 & $40^{\circ} 17^{\prime} 23^{\prime \prime}$ & $105^{\circ} 40^{\prime} 00^{\prime \prime}$ & 1.4 & 1996-2003 & 13 \\
\hline G30 & 401723105401600 & Well AGT-1 & $40^{\circ} 17^{\prime} 23^{\prime \prime}$ & $105^{\circ} 40^{\prime} 16^{\prime \prime}$ & .3 & 1996 & 2 \\
\hline
\end{tabular}

concentrations also were low and likely are derived primarily from atmospheric sources. Nitrate concentrations ranged from 0.003 to $0.22 \mathrm{mg} / \mathrm{L}$ and were substantially lower than surface water in LVWS, which may reflect longer hydrologic residence times and greater capacity for nutrient uptake by the organic-rich sediments underlying the wetland. The water quality of the water-supply wells (G1-G7) was similar in composition to the LVWS wells, except that concentrations were as much as 10 times higher. These wells are deeper than those in LVWS and likely have much longer hydrologic residence times, resulting in greater opportunity for ground water to react with aquifer materials. None of the wells had sufficient data to determine seasonal patterns or temporal trends in ground-water quality. Ground-water samples collected from LVWS did not exceed any Colorado water-supply standards. One sample from the water-supply wells exceeded the secondary standard for iron, and two exceeded the secondary standard for manganese. Secondary standards are based on aesthetic properties such as taste, odor, and staining.

\section{Summary and Suggestions for Vital Signs Monitoring}

Review of historical water-quality records identified more than 7,800 samples collected at 122 lakes, 288 streams, 
Table 33. Summary of selected water-quality data for ground-water sites in Rocky Mountain National Park, Colorado, 1971 to 2003.

[water-supply standards published by Colorado Department of Public Health and Environment (2006); <, less than; --, not reported; ${ }^{\circ} \mathrm{C}$, degrees Celsius; $\mu \mathrm{S} / \mathrm{cm}$, microsiemens per centimeter at $25^{\circ} \mathrm{C} ; \mathrm{mg} / \mathrm{L}$, milligrams per liter; $\mathrm{CaCO}_{3}$, calcium carbonate; $\mathrm{N}$, nitrogen; $\mathrm{P}$, phosphorous; $\mu \mathrm{g} / \mathrm{L}$, micrograms per liter]

\begin{tabular}{|c|c|c|c|c|c|c|c|}
\hline \multirow[b]{2}{*}{ Constituent or property } & \multicolumn{3}{|c|}{ Loch Vale wells } & \multicolumn{3}{|c|}{ Supply wells } & \multirow{2}{*}{$\begin{array}{l}\text { Water- } \\
\text { supply } \\
\text { standard }\end{array}$} \\
\hline & $\begin{array}{l}\text { Minimum } \\
\text { value }\end{array}$ & $\begin{array}{c}\text { Median } \\
\text { value }\end{array}$ & $\begin{array}{l}\text { Maximum } \\
\text { value }\end{array}$ & $\begin{array}{c}\text { Minimum } \\
\text { value }\end{array}$ & $\begin{array}{c}\text { Median } \\
\text { value }\end{array}$ & $\begin{array}{c}\text { Maximum } \\
\text { value }\end{array}$ & \\
\hline Temperature, water $\left({ }^{\circ} \mathrm{C}\right)$ & 9.0 & 9.5 & 9.7 & 5.5 & 6.0 & 9.5 & -- \\
\hline $\mathrm{pH}$ (standard units) & 5.44 & 6.31 & 6.87 & -- & -- & -- & $5.0-9.0$ \\
\hline Alkalinity $\left(\mathrm{mg} / \mathrm{L}\right.$ as $\left.\mathrm{CaCO}_{3}\right)$ & 3.3 & 5.6 & 22 & 35 & 62 & 100 & -- \\
\hline Calcium, dissolved (mg/L) & 1.7 & 2.4 & 4.5 & 11 & 16 & 30 & -- \\
\hline Chloride, dissolved (mg/L) & .02 & .14 & .43 & 2.3 & 7.0 & 23 & a250 \\
\hline Magnesium, dissolved (mg/L) & .23 & .42 & .93 & 1.9 & 2.4 & 7.3 & -- \\
\hline \multicolumn{8}{|c|}{ Other constituents } \\
\hline Nitrate, dissolved (mg/L as $\mathrm{N}$ ) & 0.003 & 0.005 & 0.22 & 0.1 & 0.2 & 1.8 & 10 \\
\hline Orthophosphate, dissolved (mg/L as P) & -- & -- & -- & $<.01$ & $<.01$ & .01 & -- \\
\hline Carbon, organic (mg/L) & 1.4 & 2.2 & 7.5 & -- & -- & -- & -- \\
\hline Iron, dissolved ( $\mu \mathrm{g} / \mathrm{L})$ & -- & -- & -- & 20 & 60 & 1,300 & a300 \\
\hline Manganese, dissolved $(\mu \mathrm{g} / \mathrm{L})$ & -- & -- & -- & 10 & 10 & 720 & ${ }^{\mathrm{a}} 50$ \\
\hline
\end{tabular}

aSecondary standard based on aesthetic properties such as taste, odor, and staining.

and 83 springs in ROMO from 1955 to 2004. More than one-half of the samples were collected at sites in LVWS for ecological research. Five streamflow-gaging stations currently (2007) are operated in the park, including Colorado River below Baker Gulch (USGS station 09010500), which began operation in 1953 and is the longest continuously operating station in the park. Surface water is a dilute calcium-sodiumbicarbonate type and dissolved solids primarily are derived from weathering of plagioclase and trace amounts of calcite in the crystalline bedrock. Nitrogen deposition rates have been increasing in the park during the past two decades, and current levels are estimated to be 15 to 20 times greater than the natural background level. Prolonged nitrogen deposition has caused nitrogen saturation resulting in chronically elevated levels of nitrate in some lakes and streams on the east side of the Continental Divide. Four sites had sufficient data to test for temporal trends in water quality. Upward trends were detected for nitrate (in three of the four sites tested) and base cations (all four sites), which were attributed to long-term increases in nitrogen deposition and declines in precipitation amount. All streams and lakes located entirely within the boundaries of ROMO are designated as Outstanding Natural Waters, which offers the highest level of protec- tion. Ground-water quality and resources in the park are not well characterized and few water-quality data are available for this resource.

The primary water-quality issue in ROMO is the effect of atmospheric nitrogen deposition on high-elevation lakes and streams. Climate change may have long-term effects on water resources in the park. Based on a review of the historical data, the following suggestions are provided for consideration in designing a water-quality monitoring plan for ROMO.

- Establish long-term water-quality monitoring stations or "sentinel sites" at different elevations to capture gradients in deposition. Big Thompson at Moraine Park (USGS station 402114105350101) is suggested as a lower elevation site. This site has a USGS streamflowgaging station and is sampled weekly to monthly as part of the USGS Hydrologic Benchmark Network for major constituents and nutrients. Andrews Creek or The Loch in LVWS is suggested as a possible highelevation sentinel site.

- Conduct periodic surveys of high-elevation lakes in the park to monitor the effects of atmospheric deposition of pollutants. 
- Conduct monitoring at selected surface-water sites to determine the effects of visitor use, park facilities, and roads on nutrients, sediment, and bacteria.

- Establish a network of streamflow-gaging stations and ground-water wells in the park to monitor the effect of climate change on water resources.

\section{Summary}

Historical data were assessed to describe water-quality conditions six National Park units that compose the Rocky Mountain Network. The park units in Colorado are Florissant Fossil Beds National Monument, Great Sand Dunes National Park and Preserve, and Rocky Mountain National Park; and in Montana they are Glacier National Park, Grant-Kohrs Ranch National Historic Site, and Little Bighorn Battlefield National Monument. This study was done in cooperation with the Inventory and Monitoring Program of the National Park Service to aid in the design of an effective and efficient waterquality monitoring plan for each park. Results for each of the parks are discussed separately in the report. Each section provides a brief description of the environmental setting of the park and an overview of the park's water resources. Statistical summaries of selected water-quality constituents are presented and compared to aquatic-life and drinking-water standards. Spatial, seasonal, and temporal patterns in constituent concentrations were examined to help identify natural and anthropogenic factors controlling water quality in each of the six parks. Suggestions are provided for consideration in designing waterquality monitoring plans. Issues of concern for water resources in the park include visitor use, land-use activities adjacent to the park, and climate change.

Florissant Fossil Beds National Monument is located on the eastern slope of the Colorado Front Range approximately 48 kilometers west of Colorado Springs, Colorado. Surface water in the area is a calcium-bicarbonate type, and dissolved solids primarily are derived from weathering of the underlying granite and lake-bed sediments. Land-use activities likely have a minor effect on water quality, although there is concern about future increases in visitor use and residential development in areas upstream from the park. Because of a lack of long-term monitoring sites, it was not possible to identify temporal trends in water quality. Water-quality data are available for one sample at each of the two ground-water wells in the park. One of the wells exceeded the drinking-water standard for fluoride and the other well exceeded secondary standards for manganese and iron. Elevated fluoride in the ground water is attributed to natural weathering of fluoride-rich bedrock that underlies the park. Issues of concern for water resources in the park include visitor use, land-use activities adjacent to the park, and climate change.

Glacier National Park is located in the northern Rocky Mountains in northwestern Montana and is bounded to the north by Canada. The North and Middle Forks of the Flathead
River border the park on the west and south, and the park is bisected by the Continental Divide. Surface water is a moderately dilute calcium-bicarbonate type, and dissolved solids primarily are derived from weathering of carbonate minerals in the sedimentary bedrock. Wildfire was determined to be an important control on stream-water nutrients in areas of the park burned during 2003. Five sites had sufficient data to test for temporal trends in water quality. The only detected trends were attributed to analytical-method-related factors, rather than environmental change. Ground-water quality and resources in the park are not well characterized, and no waterquality data are available for the park for the past 25 years. Some water-quality issues in the park include effects of visitor use and park facilities, atmospheric deposition of pollutants to high-elevation lakes, and land-use activities in areas outside the park draining the North and Middle Forks of the Flathead River. Climate change may have long-term effects on water resources in the park.

Grant-Kohrs Ranch National Historic Site is located at the north end of the Deer Lodge Valley in western Montana. One-half of the water-quality samples were collected at the Clark Fork River at Deer Lodge. Water in the Clark Fork River is a calcium-bicarbonate-sulfate type and major constituents primarily are derived from weathering of rock fragments in the alluvial fill except sulfate, which is released from oxidation of pyrite present in mine wastes. Arsenic, cadmium, copper, lead, manganese, and zinc, which are trace elements associated with mine wastes from historical mining activities, commonly were measured at detectable concentrations in the river. Nutrients in the river were mainly human-derived related to wastewater discharge and agriculture. Statistically significant downward trends detected in dissolved calcium, sulfate, and zinc in the Clark Fork River can be attributed to remediation and restoration efforts during the 1980s and early 1990s. Ground water is a calcium-bicarbonate type with moderately high dissolved solids (213 to 536 milligrams per liter). Nutrient concentrations in ground water were low, indicating minimal effects from agricultural activities in the park. Samples from 4 of the 21 ground-water wells exceeded the human-health standard for arsenic, although none of the wells are used as a water supply. Nutrient input from the sewage irrigation program and trace-element and sediment contamination related to mine tailings and their remediation are the primary water-quality issues for the reach of the Clark Fork River in Grant-Kohrs Ranch National Historic Site.

Great Sand Dunes National Park and Preserve is located along the eastern edge of the broad, flat San Luis Valley in south-central Colorado. Surface water is a calcium-bicarbonate type and dissolved solids primarily are derived from weathering of crystalline bedrock in the Sangre De Cristo Mountains. Visitor use and park facilities likely have a minimal effect on water quality, except perhaps in the lower reaches of Medano Creek. Preliminary data indicate that lakes at high elevations in the park may be in early stages of nitrogen saturation because of atmospheric nitrogen deposition. Sufficient data were available to test for temporal trends in water quality only 
for field properties at one site, which showed no trends. Waterquality issues for the park include effects of visitor use and atmospheric deposition of pollutants, maintenance of potable water supplies, and attainment of Outstanding Natural Waters designation. The primary threats to water resources in the park are surface- and ground-water withdrawals and long-term climate changes.

The Little Big Horn Battlefield National Monument is located within the Little Bighorn River Valley in southcentral Montana near the town of Crow Agency. Surface water is a calcium-bicarbonate-sulfate type, and major constituents primarily are derived from natural weathering processes. Agricultural activities upstream from the park may affect nutrient and sediment concentrations in surface water, particularly during periods of increased streamflow. Because of a lack of long-term monitoring data, it was not possible to identify temporal trends in the water quality of the river. The available water-quality data indicate the river in this reach meets Montana aquatic-life and human-health standards, except perhaps during periods of high sediment transport. The standard comparison, however, is based on relatively few samples and additional data would be needed to characterize water quality conditions during high flow. Ground water is a sodium-bicarbonate-sulfate type with elevated dissolved solid concentrations (more than 800 milligrams per liter). Nutrient concentrations in ground water were low, indicating minimal effects from agricultural activities outside the park. None of the ground-water samples exceeded human-health standards, although several exceeded the secondary standards for manganese and iron. Effects of agricultural activities outside the park on nutrient, bacteria, and sediment concentrations likely are the main water-quality issues for the Little Bighorn River.

Rocky Mountain National Park is located in the Colorado Front Range in north-central Colorado approximately 80 kilometers northeast of Denver. Surface water is a dilute calcium-bicarbonate type and dissolved solids primarily are derived from weathering of plagioclase and trace amounts of calcite in the crystalline bedrock. Nitrogen deposition rates have been increasing in the park during the past two decades, and current levels are estimated to be 15 to 20 times greater than the natural background level. Prolonged nitrogen deposition has caused nitrogen saturation resulting in chronically elevated levels of nitrate in some lakes and streams on the east side of the Continental Divide. Four sites had sufficient data to test for temporal trends in water quality. Upward trends were detected for nitrate and base cations, which were attributed to long-term increases in nitrogen deposition and declines in precipitation amount. Ground-water quality and resources in the park are not well characterized and few water-quality data are available for this resource. The primary water-quality issue in the park is the effect of atmospheric nitrogen deposition on high-elevation lakes and streams. Climate change may have long-term effects on water resources in the park.

\section{References Cited}

Bachman, S.A., 1994, Hydrology of a subalpine wetland complex in Rocky Mountain National Park, Colorado: Masters thesis, Colorado State University, Fort Collins, 146 p.

Baedecker, M.J., and Friedman, L.C., 2000, Water, energy, and biogeochemical budgets-A watershed research program: U.S. Geological Survey Fact Sheet 165-99.

Baron, Jill, 1992, Biogeochemistry of a subalpine ecosystem-Loch Vale Watershed: New York, Springer Verlag, Ecological Studies Analysis and Synthesis 90, 247 p.

Baron, J.S., 2006, Hindcasting nitrogen deposition to determine an ecological critical load: Ecological Applications, v. 16 , no. 2 , p. 433-439.

Baron, J.S., and Campbell, D.H., 1997, Nitrogen fluxes in a high elevation Colorado Rocky Mountain basin: Hydrological Processes, v. 11, p. 783-799.

Baron, Jill, and Denning, A.S., 1993, The influence of mountain meteorology on precipitation chemistry at low and high elevations of the Colorado Front Range, USA: Atmospheric Environment, v. 27, p. 2,337-2,349.

Baron, J.S., Rueth, H.M., Wolfe, A.M., Nydick, K.R., Allstott, E.J., Minear, J.T., and Moraska, B., 2000, Ecosystem responses to nitrogen deposition in the Colorado Front Range: Ecosystems v. 3, p. 352-368.

Berg, R.B., 2004, Geologic Map of the Deer Lodge and Conleys Lake 71/2' Quadrangles, Powell County, Southwestern Montana: Montana Bureau of Mines and Geology, MBMG Open File Report 509.

Boettcher, A. J., 1973, Evaluation of the ground-water supply at eight sites in Glacier National Park, northwestern Montana: U.S. Geological Survey Open-File Report 74-1013, $30 \mathrm{p}$.

Bossong, C.R., Caine, J.S., Stannard, D.I., Flynn, J.I., Stevens, M.R., and Heiny-Dash, J.S., 2003, Hydrologic conditions and assessment of water resources in the Turkey Creek Watershed, Jefferson County, Colorado, 1998-2001: U.S. Geological Survey Water-Resources Investigations Report 03-4034, $140 \mathrm{p}$.

Braddock, W.A., and Cole, J.C., 1990, Geologic map of Rocky Mountain National Park and vicinity, Colorado: U.S. Geological Survey Miscellaneous Investigations Series Map I-1973.

Brick, C., and Moore, J.N., 1996, Diel variation of aqueous trace metals in the upper Clark Fork River, Montana: Environmental Science and Technology, v. 30, no. 6, p. 1,953-1,960. 
Britten, M., Schweiger, E.W., Frakes, B., Manier, D., and Pillmore, D., 2006, Rocky Mountain Network vital signs monitoring plan: National Park Service, U.S. Department of the Interior, Natural Resource Report NPS/ROMN/NRR2007/00X.

Burns, D.A., 2004, The effects of atmospheric nitrogen deposition in the Rocky Mountains of Colorado and southern Wyoming, USA - A critical review: Environmental Pollution, v. 127 , p. 257-269.

Campbell, D.H., Baron, J., Tonnessen, K. A., Brooks, P.D., and Schuster, P.F., 2000, Controls on nitrogen flux in alpine/subalpine watersheds of Colorado: Water Resources Research, v. 36, p. 37-47.

Campbell, D.H., Clow, D.W., Ingersoll, G.P., Mast, M.A., Spahr, N.E., and Turk, J.T., 1995, Processes controlling the chemistry of two snowmelt-dominated streams in the Rocky Mountains: Water Resources Research, v. 31, no. 11, p. 2,811-2,821.

Chatman, M., Sharrow, D., and Valdez, A., 1997, Water resource management plan, Great Sand Dunes National Monument, Colorado: National Park Service, Water Resources Division, 203 p., accessed January 2007 at http:// www.nature.nps.gov/water/management_plans/GRSA_ Final_screen.pdf.

Clark, G.M., Mueller, D.K., and Mast, M.A., 2000, Nutrient concentrations and yields in undeveloped stream basins of the United States: Journal of the American Water Resources Association, v. 36, no. 4, p. 849-860.

Clark, M.L., Eddy-Miller, C.A., and Mast, M.A., 1999, Environmental characteristics and water quality of Hydrologic Benchmark Network Stations in the West-Central United States: U.S. Geological Survey Circular 1173C, 115 p.

Clow, D.W., Campbell, D.H., Mast, M.A., Striegl, R.G., Wickland, K.P., and Ingersoll, G.P., 2000, Loch Vale, ColoradoA Water, Energy, and Biogeochemical Budgets Program site: U.S. Geological Survey Fact Sheet 164-99.

Clow, D.W., Mast, M.A., Bullen, T.D., and Turk, J.T., 1997, ${ }^{87} \mathrm{Sr} /{ }^{86} \mathrm{Sr}$ as a tracer of mineral weathering reactions and calcium sources in an alpine/subalpine watershed, Loch Vale, Colorado: Water Resources Research, v. 33, no. 6, p. 1,335-1,351.

Clow, D.W., Schrott, L., Webb, R., Campbell, D.H., Torizzo, A., and Dornblaser, M., 2004, Ground water occurrence and contributions to streamflow in an alpine catchment, Colorado Front Range: Ground Water, v. 41, no. 7, p. 937-949.

Clow, D.W., Sickman, J.O., Striegl, R.G., Krabbenhoft, D.P., Elliott, J.G., Dornblaser, M.M., Roth, D.A., and Campbell, D.H., 2003, Changes in the chemistry of lakes and precipitation in high-elevation National Parks in the Western
United States, 1985-99: Water Resources Research, v. 39, no. 6, 1171, doi: 10.1029/2002WR001533.

Clow, D.W., Striegl, R.G., Nanus, L., Mast, M.A., Campbell, D.H., and Krabbenhoft, D.P, 2002, Chemistry of selected high-elevation lakes in seven National Parks in the Western United States: Water, Air, and Soil Pollution Focus, v. 2, p. 139-164.

Clow, D.W., and Sueker, J.K., 2000, Relations between basin characteristics and solute concentrations in alpine/subalpine basins in Rocky Mountain National Park, Colorado: Water Resources Research, v. 36, p. 49-61.

Colorado Department of Public Health and Environment, 2003, Surface water quality classifications and standardsRegulation 36-Classification and numeric standards for Rio Grande Basin: Colorado Department of Public Health and Environment Water Quality Control Division, Denver, Colo., accessed January 2007 at http://www.cdphe.state. co.us/regulations/wqccregs/index.html.

Colorado Department of Public Health and Environment, 2006, Surface water quality classifications and standardsRegulation 38-Classification and numeric standards for South Platte River Basin, Laramie River Basin, Republican River Basin, Smoky Hill River Basin: Colorado Department of Public Health and Environment Water Quality Control Division, Denver, Colo., accessed January 2007 at http:// www.cdphe.state.co.us/regulations/wqccregs/index.html.

Cooper, D.J., MacDonald, L.H., Wenger, S.K., and Woods, S.W., 1998, Hydrologic restoration of a fen in Rocky Mountain National Park, Colorado, USA: Wetlands, v. 18, no. 3, p. 335-345.

Dodge, K.A., Hornberger, M.I., and Dyke, J.L., 2005, Waterquality, bed-sediment, and biological data (October 2003 through September 2004) and statistical summaries of data for streams in the upper Clark Fork Basin, Montana: U.S. Geological Survey Open-File Report 2005-1356, 124 p.

Ellis, B.K., Stanford, J.A., Craft, J.A., Chess, D.W., Gregory, G.R., and Marnell, L.F., 1992, Monitoring water quality of selected lakes in Glacier National Park, Montana-Analysis of data collected, 1984-1990: University of Montana, Flathead Lake Biological Station Open-File Report 129-92.

Ellis, B.K., Stanford, J.A., Craft, J.A., Chess, D.W., Hauer F.R., and Whited, D.C., 2003, Plankton communities of alpine and subalpine lakes in Glacier National Park, Montana, U.S.A., 1984-1990: Verhandlungen der Internationalen Vereinigung für Theoretische und Angewandte Limnologie, v. 28, no. 3, p. 1,542-1,550.

Fagre, D.B., Running, S.W., Keane, R.E., and Peterson, D.L., 2005, Assessing climate change effects on mountain ecosystems using integrated models-A case study, in Huber, U.M, Bugmann, H.K., and Reasoner, M.A., eds., Global 
change and mountain regions-A state of knowledge overview: Kluwer Academic Press, Dordrecht, The Netherlands, p. $455-466$.

Ferguson, S.A., 2003, Investigation of water quality in the Great Sand Dunes National Monument and Preserve, Saguache County, Colorado, February 1999 through September 2000_Qualifying for Outstanding Waters Designation: U.S. Geological Survey Water-Resources Investigations Report 02-4196.

Finklin, A.I., 1986, A climatic handbook for Glacier National Park with data for Waterton Lakes National Park: U.S. Department of Agriculture Forest Service General Technical Report INT-204.

Fishman, M.J., Raese, J.W., Gerlitz, C.N., and Husband, R.A., 1994, U.S. Geological Survey approved inorganic and organic methods for the analysis of water and fluvial sediment, 1954-94: U.S. Geological Survey Open-File Report 94-351, $55 \mathrm{p}$.

Galloway, J.N., Norton, S.A., and Church, M.R., 1983, Freshwater acidification from atmospheric deposition of sulfuric acid-A conceptual model: Environmental Science and Technology, v. 17, p. 541-545.

Gibson, J.H., Galloway, J.N., Schofield, C., McFee, W., Johnson, R., McCarley, S., Dise, N., and Herzog, D., 1983, Rocky Mountain Acidification Study: U.S. Fish and Wildlife Service, Division of Biological Services, Eastern Energy and Land Use Team, FWS/OBS-80/40.17, 137 p.

Hauer, F.R., Fagre, D.B., and Stanford, J.A., 2003, Hydrologic processes and nutrient dynamics in a pristine mountain catchment: Verhandlungen der Internationalen Vereinigung für Theoretische und Angewandte Limnologie, v. 28, no. 3, p. 1,490-1,493.

Hauer, F.R., Stanford, J.A., and Lorang, M.S., 2007, Pattern and process in northern Rocky Mountain headwatersEcological linkages in the headwaters of the Crown of the Continent: Journal of the American Water Resources Association, v. 43, no. 1, p. 104-117.

Hawley, C.C., and Wobus, R.A., 1977, General geology and petrology of the Precambrian crystalline rocks, Park and Jefferson Counties, Colorado: U.S. Geological Survey Professional Paper 608-B, 77 p.

Hem, J.D., 1985, Study and interpretation of the chemical characteristics of natural water: U.S. Geological Survey Water-Supply Paper 2254, 263 p.

Hirsch, R.M., Slack, J.R., and Smith, R.A., 1982, Techniques of trend analysis for monthly water-quality data: Water Resources Research, v. 18, no. 1, p. 107-121.

Hornberger, M.I., Lambing, J.H., Luoma, S.N., and Axtmann, E.V., 1997, Spatial and temporal trends of trace metals in surface water, bed sediment, and biota of the upper Clark Fork basin, Montana, 1985-95: U.S. Geological Survey Open-File Report 97-669, 84 p.

Johnson, M.M., 2002, The fate of sewage effluent nutrients applied to coarse-grained soils by sprinkler irrigation: Masters thesis, University of Montana, Missoula, 97 p.

Konizeski, R.L., McMurtrey, R.G., Brietkrietz, A., 1962, Preliminary report on the geology and ground-water resources of the southern part of the Deer Lodge Valley, Montana: Montana Bureau of Mines and Geology Bulletin 31, 24 p.

Lambing, J.H., 1991, Water-quality and transport characteristics of suspended sediment and trace elements in streamflow of the upper Clark Fork basin from Galen to Missoula, Montana, 1985-90: U.S. Geological Survey WaterResources Investigations Report 91-4139, 73 p.

Landers, D.H., Eilers, J.M., Brakke, D.F., Overton, W.S., Kellar, P.E., Silverstein, M.E., Schonbrod, R.D., Crowe, R.E., Linthurst, R.A., Omernik, J.M., Teague, S.A., and Meier, E.P., 1987, Characteristics of lakes in the Western United States. Volume 1. Population descriptions and physicochemical relationships: U.S. Environmental Protection Agency, Washington, D.C., EPA/600/3-86/054a, 176 p.

Madole, R.F., 1976, Glacial geology of the Front Range, Colorado, in Mahaney, W.C., ed., Quaternary Stratigraphy of North America: Dowden, Hutchinson, \& Ross, Stroudsburg, p. 297-318.

Mast, M.A., Campbell, D.H., Krabbenhoft, D.P., and Taylor, H.E., 2005, Mercury transport in a high-elevation watershed in Rocky Mountain National Park, Colorado: Water, Air, and Soil Pollution v. 161, p. 21-42.

Mast, M.A., Foreman, W.T., and Skaates, S.V., 2006, Organochlorine compounds and current-use pesticides in snow and lake sediment in Rocky Mountain National Park, Colorado, and Glacier National Park, Montana, 2002-03: U.S. Geological Survey Scientific Investigations Report 2006-5119, $54 \mathrm{p}$.

Mast, M.A., Kendall, C., Campbell, D.H., Clow, D.W., and Back J., 1995, Determination of hydrologic pathways in an alpine-subalpine basin using isotopic and chemical tracers, Loch Vale Watershed, Colorado, in Tonnesson, K.A., Williams, M.W., and Tranter, M., eds., Biogeochemistry of Seasonally Snow-Covered Catchments: International Association of Hydrologic Sciences Press, Publication No. 228, Institute of Hydrology, Wallingford, Oxfordshire, UK, p. $263-270$.

Mast, M.A., and Turk, J.T., 1999, Environmental characteristics and water quality of hydrologic benchmark network stations in the Eastern United States: U.S. Geological Survey Circular 1173-A, 158 p. 
Moore, J.N., and Woessner, W.W., 2000, Geologic, soil water and groundwater Report-Grant Kohrs Ranch National Historic Site: Technical Report to National Park Service, University of Montana, accessed on January 2007 at $h t t p: / /$ science.nature.nps.gov/nrdata/datastore.cfm?ID $=38370$.

Moreland, J.A., and Wood, W.A., 1982, Appraisal of groundwater quality near wastewater-treatment facilities, Glacier National Park, Montana: U.S. Geological Survey WaterResources Investigations Report 82-4, 27 p.

Mueller, D.K., Hamilton, P.A., Helsel, D.R., Hitt, K.J., Ruddy, B.C., 1995, Nutrients in ground water and surface water of the United States; an analysis of data through 1992: U.S. Geological Survey Water-Resources Investigation 95-4031, $74 \mathrm{p}$.

Mueller, D.K., and Helsel, D.R., 1996, Nutrients in the Nation's waters-Too much of a good thing?: U.S. Geological Survey Circular 1136, 24 p.

Murdoch, P.S., McHale, M.R., Mast, M.A., Clow, D.W., 2005, The U.S. Geological Survey Hydrologic Benchmark Network: U.S. Geological Survey Fact Sheet 2005-3135.

Nanus, Leora, 2005, Predicting Deposition-Sensitive Lakes in Rocky Mountain National Parks using Geographic Information System and Logistic Regression: Abstracts with Programs, American Association of Geographers.

Nanus, Leora, Campbell, D.H., and Williams, M.W., 2005, Sensitivity of alpine and subalpine lakes to acidification from atmospheric deposition in Grand Teton National Park and Yellowstone National Park, Wyoming: U.S. Geological Survey Scientific Investigations Report 2005-5023, 37 p.

National Park Service, 1998, Baseline water quality data inventory and analysis-Florissant Fossil Beds National Monument: National Park Service, Water Resources Division, Technical Report NPS/NRWRD/NRTR-98/173.

National Park Service, 2005, Park vital signs monitoringtaking the pulse of the national parks. Natural Resource Program Center Office of Inventory, Monitoring and Evaluation National Park Service, accessed on January 2007 at http://science.nature.nps.gov/im/monitor/docs/Monitoring_ Brochure.pdf.

National Park Service, 2006, Florissant Fossil Beds National Monument, Geologic Resource Evaluation Report: National Park Service, Geologic Resources Division, NPS D-37, 35 p., accessed on January 2007 at http://www2.nature.nps. gov/geology/inventory/publications/reports/flfo_gre_rpt_ view.pdf.

Nimick, D.A., 1993, Hydrology and water chemistry of shallow aquifers along the upper Clark Fork, Western Montana: U.S. Geological Survey Water-Resources Investigations Report 93-4052, 63 p.
Nimick, D.A., 2003, Diurnal variation in trace-metal concentrations in streams: U.S. Geological Survey Fact Sheet FS-086-03, 4 p.

Porter, E., Blett, T., Potter, D.U., and Huber, C., 2005, Critical loads for atmospheric deposition of nitrogen and sulfur: BioScience, v. 55, no. 7, p. 603-612.

Ranalli, A.J., 2004, A summary of the scientific literature on the effects of fire on the concentration of nutrients in surface waters: U.S. Geological Survey Open-File Report 2004-1296, 23 p.

Ross, C. P., 1959, Geology of Glacier National Park and the Flathead region, northwestern Montana: U.S. Geological Survey Professional Paper 296, 125 p.

Rupert, M.G., and Plummer, L.N., 2004, Ground-Water Flow direction, water quality, recharge sources, and age, Great Sand Dunes National Monument, South-Central Colorado, 2000-2001: U.S. Geological Survey Scientific Investigations Report 2004-5027, 28 p.

Schertz, T.L., Alexander, R.B., and Ohe, D.J., 1991, The computer program Estimate Trend (ESTREND)—A system for the detection of trends in water-quality data: U.S. Geological Survey Water-Resources Investigations Report 91-4040, $60 \mathrm{p}$.

Sharp, R.R., Jr., and Aamodt, P.L., 1978, Field procedures for the uranium hydrogeochemical and stream sediment reconnaissance as used by the Los Alamos Scientific Laboratory: Los Alamos, New Mexico, Manual LA-7054-M, GJBX-68, 64 p.

Smith, J.D., Lambing, J.H., Nimick, D.A., Parrett, Charles, Ramey, Michael, and Schafer, William, 1998, Geomorphology, flood-plain tailings, and metal transport in the upper Clark Fork valley, Montana: U.S. Geological Survey WaterResources Investigations Report 98-4170, 56 p.

Sueker, J.K., Clow, D.W., Ryan, J.N., and Jarrett, R.D., 2001, Effect of basin physical characteristics on solute fluxes in nine alpine/subalpine basins, Colorado, USA: Hydrologic Processes, v. 15, p. 2,759-2,769.

Sueker, J.K., Ryan, J.N., Kendall, C., and Jarrett., R.D., 2000, Determination of hydrologic pathways and sources of water during snowmelt using two- and three-component hydrograph separations for six headwater basins, Rocky Mountain National Park: Water Resources Research, v. 36, p. 63-76.

Sullivan, T.J., Cosby, B.J., Tonnessen, K.A., and Clow., D.W., 2005, Surface water acidification responses and critical loads of sulfur and nitrogen deposition in Loch Vale watershed, Colorado: Water Resources Research, v. 41, no. 1, doi 10.1029/2004WR003414.

Tuck, L.K., 2003, Ground-water resources along the Little Bighorn River, Crow Indian Reservation, Montana: U.S. 
Geological Survey Water-Resources Investigations Report 03-4052, 45 p.

Vuke, S.M., Wilde, E.M., Lopez, D.A., Bergantino, R.N., 2000, Geologic map of the Lodge Grass 30' x 60' quadrangle, Montana: Montana Bureau of Mines and Geology, Geologic Map 56, scale 1:100,000.

White, J.D., Running, S.W., Thornton, P.E., Keane, R.E., Ryan, K.C., Fagre, D.B., and Key, C.H., 1998, Assessing simulated ecosystem processes for climate variability research at Glacier National Park, USA: Ecological Applications, v. 8 , no. 3 , p. 805-823.

Williams, M.W., Baron, J.S., Caine, N., Sommerfeld, R., and Sanford, R., 1996, Nitrogen saturation in the Rocky Moun- tains: Environmental Science and Technology, v. 30, no. 2, p. 640-646.

Wobus, R.A., and Epis, R.C., 1978, Geologic map of the Florissant 15 minute quadrangle, Park and Teller Counties, Colorado: U.S. Geological Survey Miscellaneous Geologic Investigations Map I-1044.

Woods, S.W., MacDonald, L.H., and Westbrook, C.J., 2006, Hydrologic interactions between an alluvial fan and a slope wetland in the Central Rocky Mountains: Wetlands, v. 26, no. 1, p. 230-243.

Wurstera, F.C., Cooper, D.J. and Sanforda, W.E., 2002, Stream/aquifer interactions at Great Sand Dunes National Monument, Colorado: influences on interdunal wetland disappearance: Journal of Hydrology, v. 271, p. 77-100.

Publishing support provided by:

Rolla Publishing Service Center

For more information concerning this publication, contact:

Director, U.S. Geological Survey

Colorado Water Science Center

Denver Federal Center

Box 25046, MS 415

Lakewood, CO 80225

(303) 236-4912

Or visit the Colorado Water Science Center Web site at:

http://co.water.usgs.gov 



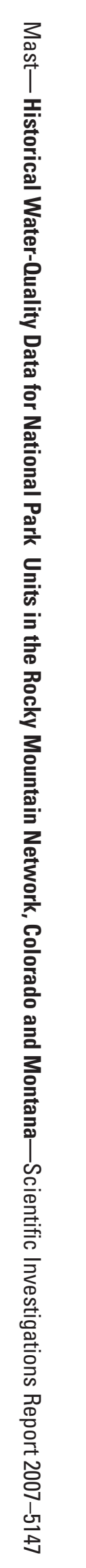

